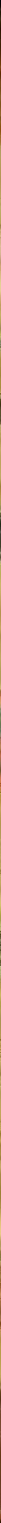




$$
\text { Or. } 19^{2}
$$


$4<21$. 



\title{
Sgippologifiche
}

\section{8 a} in

\section{Soัyrien แแก Der Witte.}

\author{
\$on
}

\section{Rouis Dambifeau,}

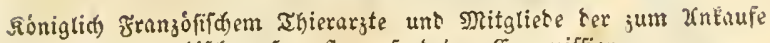
arabifaer Scengfte gefenteten Eommiffion.

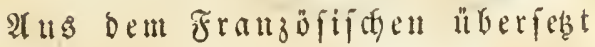

yon

Theodor Seinze,

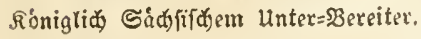

(Ẽfter Theil.

Exip3ig,

Berling you Dirzer uno vigigato.

1842. 


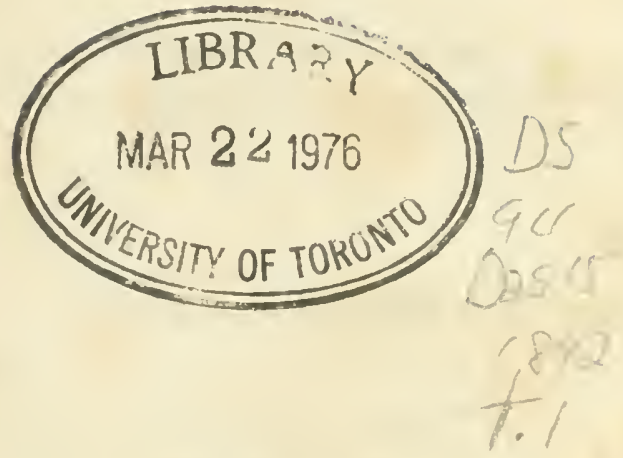




\title{
Eeiner Saodidoblgeboren
}

\author{
tem
}

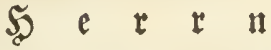 \\ Friedridy gofeph artun vou Fabice,}

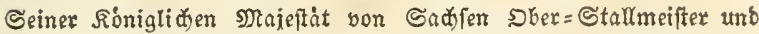

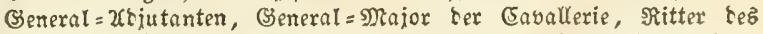

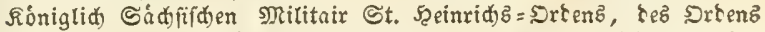

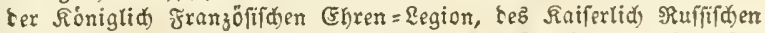

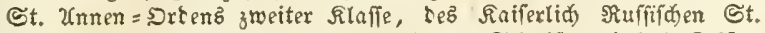

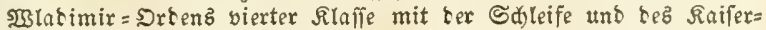
lid) Ruffifden St. Staniblaus = Drtenz exfter Rlalle, Commanteur

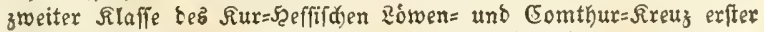

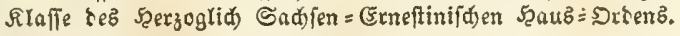

\section{in (Egrfurdyt und $\mathfrak{U n t e r t b a ̈ n i g f e i t ~}$}





\section{Scodwoflgeborner Serr,}

Söd ft 3u verefrenter Serr Dber= Stall= meifter un (S) ueral= Dajor!

Cw. Sgodwofigeboren haben mir wohlwolfento gejtattet, Shnen bieje Meberjeb̧ung zuzucignen. SBemt iaf in biejer Crrantenip einen bejonderen Beweis vont

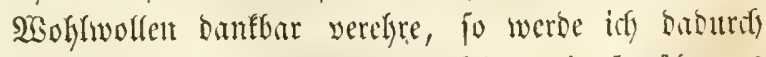
won menem frettoig antgeregt, Sgre mir fo itberants

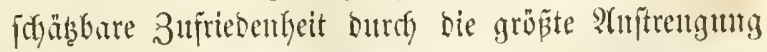
aller meiner firåjte inmer megr unto mehr zu wer= bienten. 


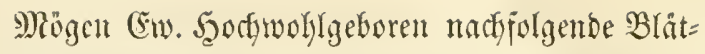
ter nachfirfytig aupnefymen, utno barin wenigftens ben

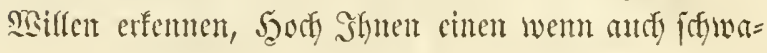
(j)en Beweis wou bon Streben an Den Tag zu Iegen, selfien 3iet bie möglidfit alljettige Wervolffommung

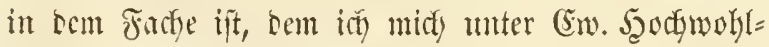
geboren Bejelyen wiome.

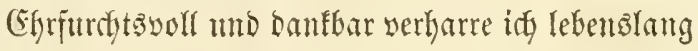

\title{
(Ew. Şorfwohlgeboren
}

\author{
Drescen, \\ am 2. Fecruar $1 \$ 42$.
}




\section{3orwort bes llebericteri.}

92ur meine Ricbe jut framöurictyen Epraffe, fo wie

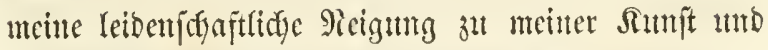
Bifijeridant fadten ben Bebanten in mir an, biejez Werf, bas mir won mefyrerm geaffetem Reitern unt Şippologen genüfmt worben war, fowofgl zt mesher

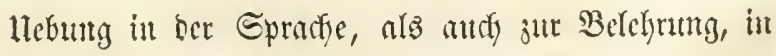

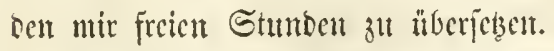

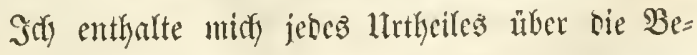

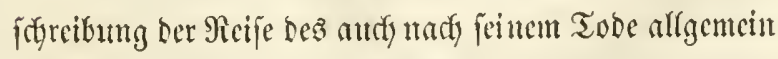

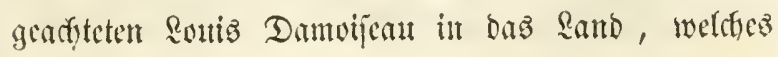




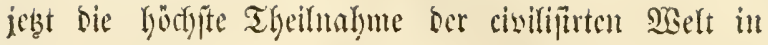

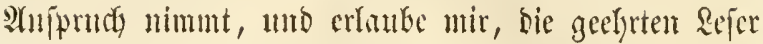

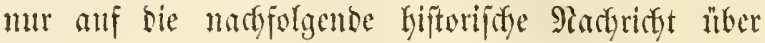

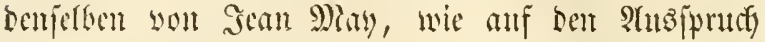

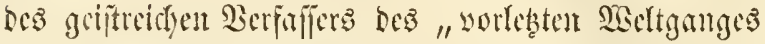

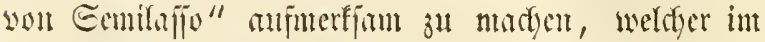
breizelyntent Bricfe unter Sflocem Folgentes fagt:

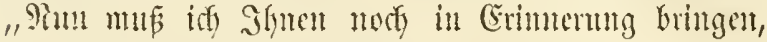

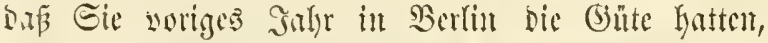
mir Dantvifent's intereffanten Beridyt feimer, im $\mathfrak{G S}_{\mathrm{b}}=$

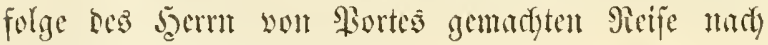
Syrien ju Yeifen, chne Extrift, weldye mit ciner Menge widytiger Plotigen fapt bas Snterefie cincs Piomans verbinhet."

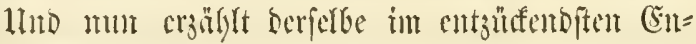

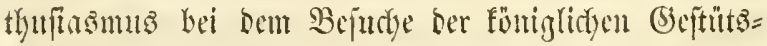

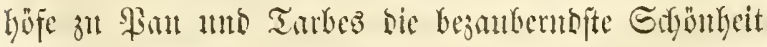
Der nod) lebemben Bafdäter won ber Ricferming tor

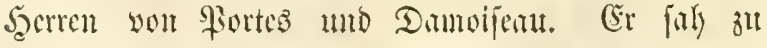

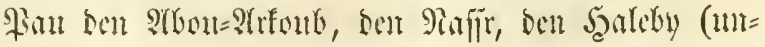

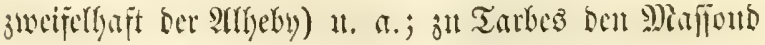

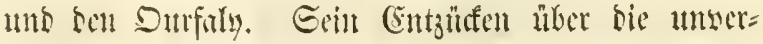


gleidflidyen Eigenffyaften und wunbervolfen Formen biefer \$ferbe, weldyez mit ber fo gewanbten Feber geffirtoert ift, ïberlajie und rathe idf jebem ber ges elyrten Rejer in bem oben bejeidyneten $\mathfrak{B}$ erfe auf=

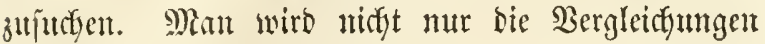

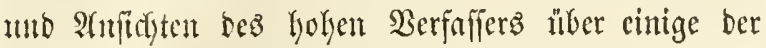
in biejem Buffe sorfommenten Sengfte fefyr anjiehent finten, fontern wirb fid auty bur(t) bas barauf fol=

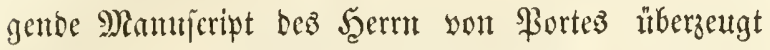

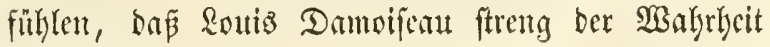
getrett war.

Sa) wollentete biefe lleberjetsung, ofhe irgent

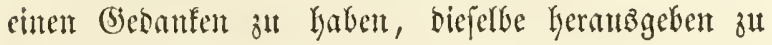
wollen, und mur ben bringenden Fufforberutngen mei= ner Freuthe zut genthgen, "ubergebe idy, nidyt ohne cin gemifię zaghaftez Befüly, biefe 3eilen ber Deffent=

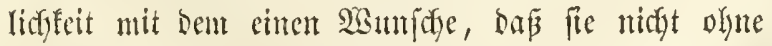
eintiges Suterefie gelejen werben mödyten, weldyes mir

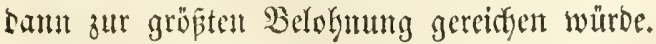

Dreşen, Den 15. December 1841.

\section{Sheodor Scinge.}





\section{Şiftorifice Pachricht ůber}

\section{Rouiz Damoifenu.}

Dięев Şerf, movon fo vicle Rapitel mit fo vielem Intereñe in bem Journal des harras, Sem Voleur umb bem Cabinet de Lecture gelejen morben find, erffjeint

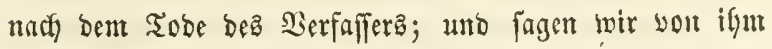

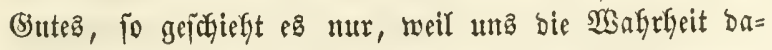

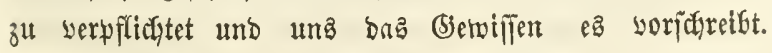
Damoifeau ift nidyt melgr; er fonmmt nicht, umjer

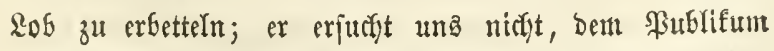
vorzulügen, um fein Buch befijer zu verfaufen ober $i(j)$

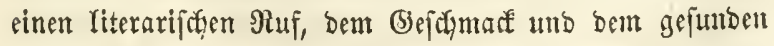
Menfajenverftande zum Trokge, zu begrïtuben; fein $\mathfrak{B}_{\ell}=$ meggruno, fein 厄inflú fonnte unz aljo beftimnten, ben

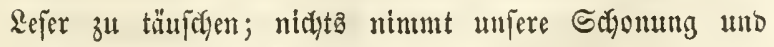

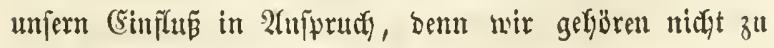


benen, weldye ben vorzugbetwe als mafr angenommenen

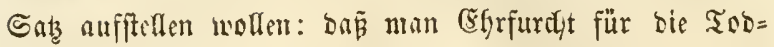
ten Yaben foll marum bent bieje (sforfurcht wor ifgrem Etaube, ba man ifnen nirjyt inefyr bas geringfte ltnredjt Jufügen famm, ba fie ber Bernibutung angefören? Noem

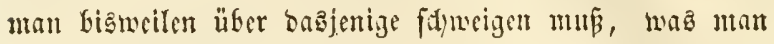
won Refentoen benft, un benfelben nicht ju ferjaben, fo

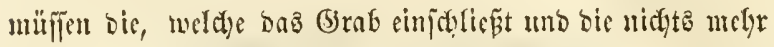

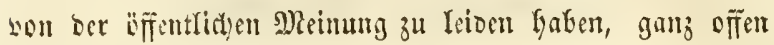

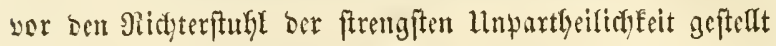
nerben.

Souis Damsifcan, beffen Brtern menig yom Glilut begünftigt marest, erbieft nur cine wenig umfafiende (Er

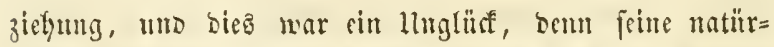
Yid)en Jägigfeiten, surch gefditftere $2 e$ frer entwiffeit, alb er in feiner Stigend hatte, würben ifgn unfehlbar weit über ben Stanofunft erboben faben, nuf meldyem er geblieben ift. Mlit $\mathfrak{B e r j t a n d}$ ausgeftattet umb nla vernïnftiger scobadyter miomete or fitd frillyeitig ber Thierbcilfunde, mozn er mur ber Renutnif́ feiner 9lutterfpracife beourfte,

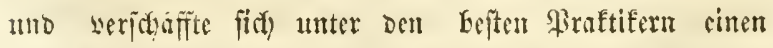

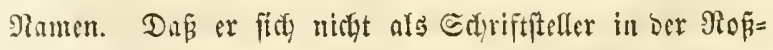
arzneifunde bofammt madute, Daran verfinderte ifhn mur

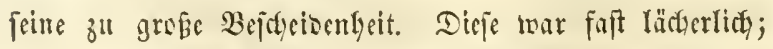
benn er fürdytete, fich zu fdyledyt nuşzubritifen, um $\mathfrak{g} e=$ refen zul meroen, obgleidy Man fitd überzengen wiro,

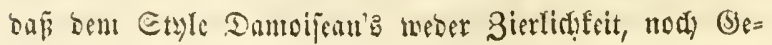


mauigfeit felylt. (5r gift bas, maz or gejefen bat, auf cine Feftimmte, furz gefapite unt oramatifitye sgeije nieber, shne Effect madyen zu mollen; er erzäglt feine Erfabrum= gen mit oem Talent, fte mitzutbeilen, unto oors marem

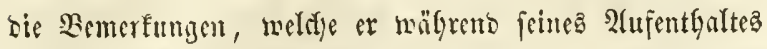
unter ben 2tratern madute, nur füt ifyn feleft, bemn or batute feineswegg Daran, fite in ein Şerf zu vereinigen,

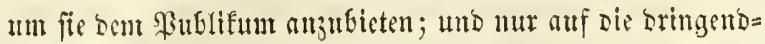
fiten Bitten feiner Frembe entict) of er fict soju. SBir fömten befaupten, Dá man Daran 2 ergntigen finben srirb,

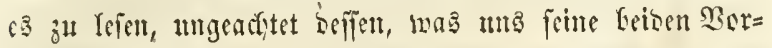

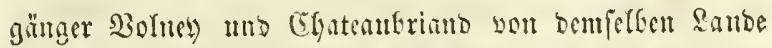

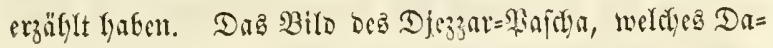

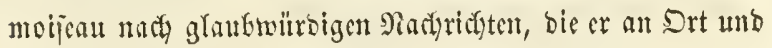
Etefle foumelte, gezeidnet hat, ift ein felyr merfmürsiges

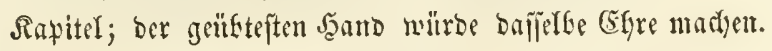

Dantoijeau farb an einer Eingeweid = Entjïnbung, lantge Seit wor ben Biele, weldes ifyut bie latur be= fimmt zu Gaben ferjien; berm. er war von ber ftärffiten

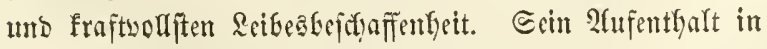

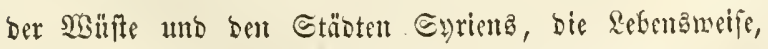
welche or bort zu filfren geznumgen war, ergengte in ifm baz 1tebel, defijen Gewalt er fuäter unterlag. Drei= jägrige Seiben und (5efalyren fern won feinem Baterlande

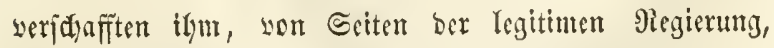
fein anderes Beidyen oer Danffurfit, als bas 2tnerbieten

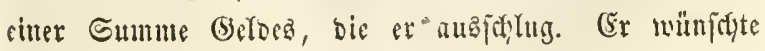


fta) Das నreuz ber Shrenlegion; aber zu würbig und zu

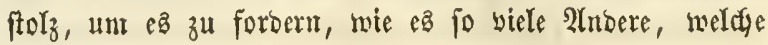

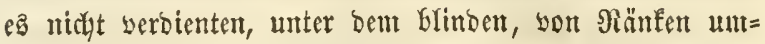
lagerten Sarl $X$. getfaur Gatteu, fah ex Yteber, bá biejer Monardy bie Ungeredytigfeit beging, feine Brutt nidyt $b a=$

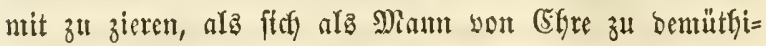
gentsen Bemerbungen zu erniebrigen.

Souis Damoifeau niro yon aflen benen bebauert meroen, bie ifn gefamut laben; jeine Freumbe fünnen fid ïber feinen Serlujt nidjt tröften; er Gatte fitd beren burds jeinen woblmollenben, einuelyntentoen unb ganz freintütyi=

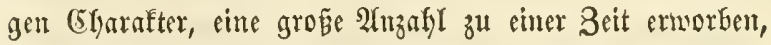
wo inan ber Selbffudyt unb ber Hurrebliduteit an bäu= r̈gften unter ben Menjofen begegnet.

\section{Sean Mzay.}




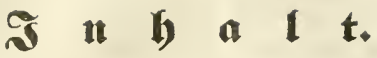

Seite

Bueignung. , . . . . . . . . . , III-VI

Borwort tez นeberfę̧erz. . . . . . . . vil $-\mathrm{x}$

Şiftorif

\section{Exites Sapitel.}

Borlåufige Betradtungen. - ZCbreife von Marfeille. - 2CnEunft in 2Clexantrette (Scanterum). - Der Srontez. - Ebene von Zntiodien. - Turfoma= nen. - Kinterblattern; sie (Einimpfung aller אinter eines Bolkzftammeb. - Der Berg St. Simon. - 2(nkunft

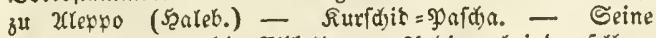
Serađtung gegen bie Etikette. - 2utienz bei semfelken. - Bewunterung Ser Drientaten fír Stapoleon. - Ei= gene Berlegenkeit. - Befdente. - Lunfall, welder

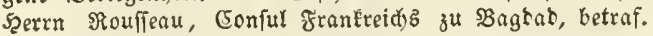
- Meine furen. - כd werbe von 2rnauten ange= griffen; sie Beftrafung berfelben. - Xnkauf bes urheby un๖ Des Sutheif.

\section{3weites $\Omega$ apitel.}

Eaftration bei Sen 2rabern. - Serwókntide Folgen ihrex

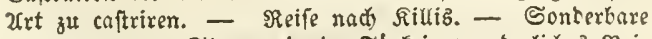

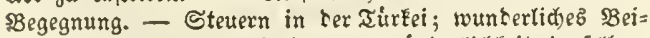

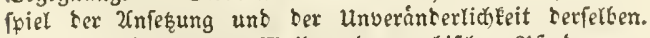

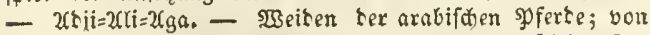
Ser 2rtt, wie fie angebunten werלen. - Begenfeitige (Se=

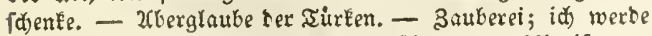
serferben bofdulsigt. - Eataverfanau. - 2ubreife von

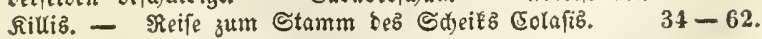

\section{Drittes $\mathfrak{x} \mathfrak{x}$ itel.}

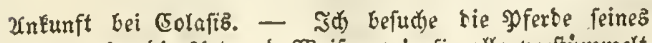

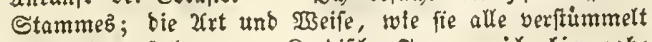

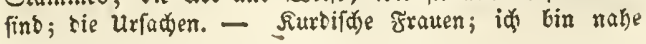




\section{XYI}

taxan, von einigen unter innen keraukt zu werten. Sameelkutter; fie 20rt, foldse zu Eereiten. - Id) ver=

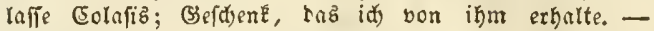

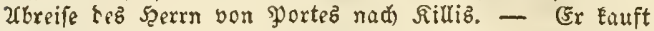

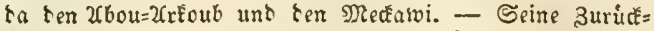
Eunft. - Serumirente Şunte ter ti̊rfirchen Ståte; ihre (Sewohnheiten. - Bergeblider Dergiftungbeverfud) an einem terfeteen. - Sonterbare Wrirkung teb Duedfil = ber=Sublimatż. - Uebung mit rem Dierio (fleiner $\mathfrak{W} 3$ urf =

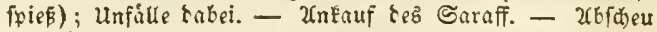

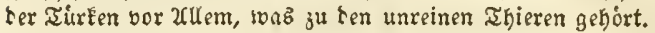

\section{3ictes Sapitel.}

Türtifđe Frauen. - (5z gelingt mix, in taz (semnd bon sreien berfetken einjutringen. - Untauf tes Tatmor, teg Drcan unt tes Durfati. - Nehre Dură Sen Reętern berurfadte Borfálle. - Die Xufunft Duhai"ż, Sibeifz

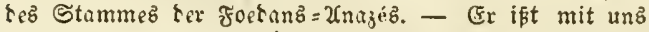

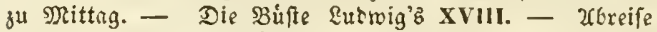
von Zleppo in tie Wruffte. - Die Souaiq (אuaik). Ruinen. - J̧ik̨e in Ser ȚJifte; Ruftipiegelung. - Unfere

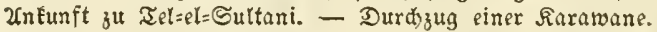

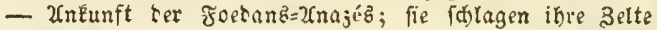
auf; befontere umftante hierbei. - Dffenherzigkeit tez

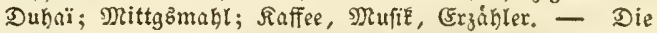
Franjofen in Xegypten. - Urbreife też Scerrn von $\Re_{3}=$ mouizłi nad) গ̧alıngra.

\section{Fü ütes Sạitel.}

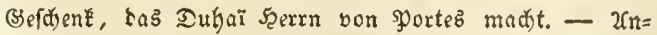

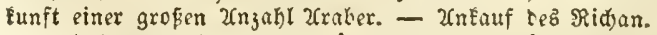

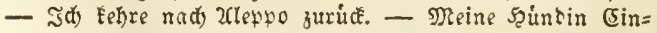

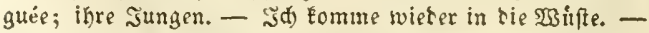
Erwerbung tes gétéan. - Franke; Şeilungen. - Daz Brennen mit rem Eifen bei ben Zrabern. - Man ftiehlt mir meine Zanze. - ZnEauf teb Maffout. - Diebftaht im turfomanifden Lager; noihere Uunftinte; bie geftohle= ner Begenftảnte werten vermittelf Lófegeto zurưdégege= ben. - Unannehmlidkeit mit Duhaï. - Seerr bon sportę berlást tie wsurte. 


\section{XVII}

\section{Scdittes Sapitel.}

Inlunft einer neuen Iruppe Turfomanen. - Radtfene.

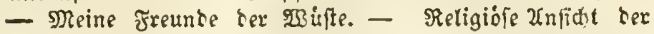
Xraker uber bie Rade; blutigez Beifpiel riefer Eeiten=

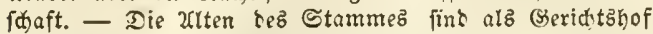
verfammelt. - urtheil. - Esfräigkét rer Zrater. Die Surfomanen bereiten fid zur 2chreife vor. - $230 r t=$ ftreit mit Duhai. - 3ufammentreffen mit sem Ubou= Эhaar. - Das selegen bei ten Jrabern. - Ja) faid mid) an, abzureifen. - Entlid) erlangter znkauf bes ubou = sphaar.

\section{Sicbeutes Sawitel.}

Xkgang von Tel=el=Sultani. - Dorfälle uno Sefahren in Ser TुSufte. - Unkunft zu Xleppo. - Der Graf von Rzeroutżi und ter sitter SBable. - Rrante spferbe, burd) Sampfbiber hergeftellt. - 2rnfunft einer nad Meffa gehenten berfiften Karawane. - Ihre Spferte. - Solle; bie 2rt, wie biefelben eingefortert werten. - Suhaï unb Daub in 2lleppo. - Berweigerter 3roeifampf. - Serr (Sontolphy, sifdof vom \&ibanon; jein Einzug in bie Statt. - Der Surfaly befinftigt fith Surd ten Saud

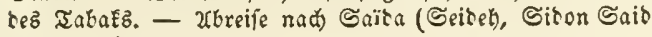
ober (Siib).

\section{Sthtes Rapitel.}

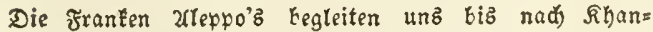

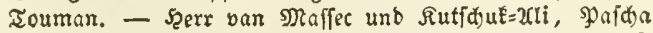

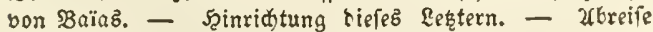

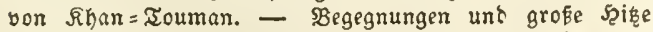

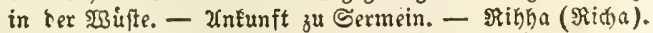
- Diibr; Saltfeunbidaft ber Tirfen bajelbit. - $\mathfrak{R} a=$ frantii; heitnifde 2unfarier. - Sturz in einen $2 \mathfrak{C b}=$ grunb; id bredse sas Sruftbein. - 3olhaus bon (5ha= far. - Das Sebirge Setfub. - Sebloulie. - 2nfunft zu Latafia; Uufenthalt bapelbit. - Neudelmorb einez jungen Ebriften; fein sob u. f. to. 



\section{Erites sapitel.}

Sortáfige Setradtungen. - Ubreife von Marfeille. - Enpern. - Znkunft in Zcterantrette (ङanterun). - Der Dronteb. Ebene von Xatiodien. - Iutfomanem. - Sinterblattern; Sie Einimffung alter finter einez ミolezftammez. - Der Setg St.

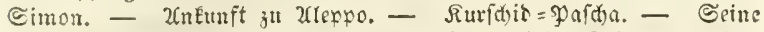
Beraditung gegen tie Ettikette. - Zutienz bei temfelben. - Be= wunterung ter Drientalen fir Rapoleon. - Eigene Berlegentheit. - Beftrenfe. - Unfau, wetder Seern Rouffeau, Eonful

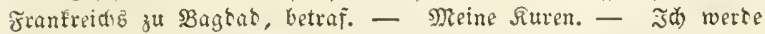
von Xrnatten angegriffen; tie seftrafung terfelben. - Unfauf tez 2ctheby und bes sutheif.

Sint Эafre 1818 mollte Serr Rainé, bamala Mini=

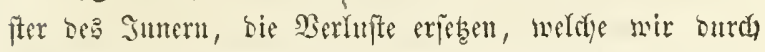

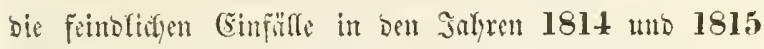
an orientalijefen Scengften erfitten fatten; bafer gaf er

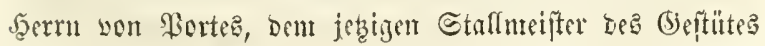

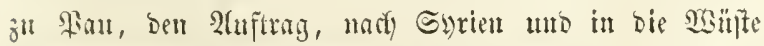
ju reifen, un etwa vierzig arafijale Bejdyäler zu faufen. S4) nutre ifm als Thierargt Feigegeben.

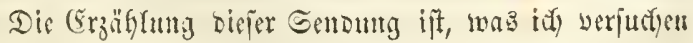

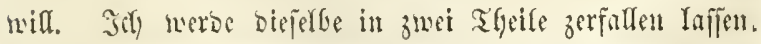
Der erfte Theil wirb Das Materielle unjerer gieife, went

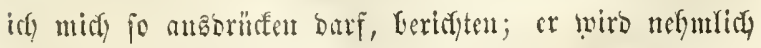
won alfen Gefoubren llmftänben, bie fith anf unjeren 3 mg 


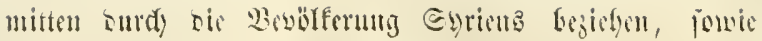

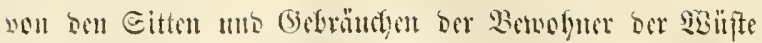
uns won allen 2iorfällen Ganbeln, weldye wir mälyeno nuferes 2 nfentfyaltes unter jenen erlebt baben.

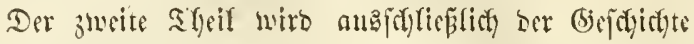

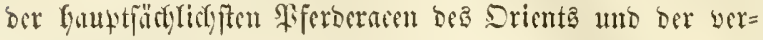
finicbenen sirusurgen, weldye fie erlitten faben, fermer

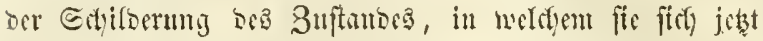

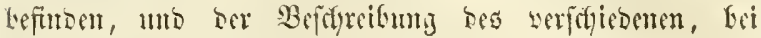

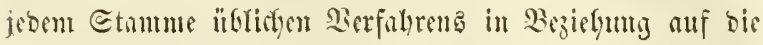

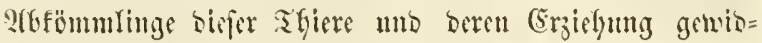
net feits.

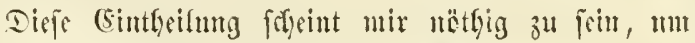
oer llnorsumg vorjubengen, neldye notguenoigermeije cine

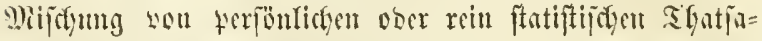

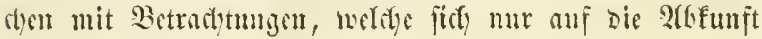

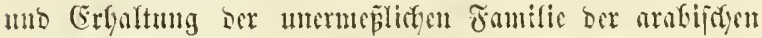

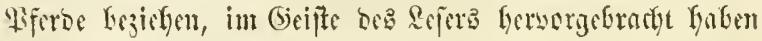

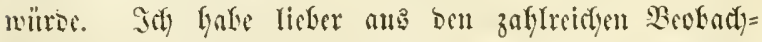
tumgen, meldae idy üfer ben lestern Gegenftand eimgefaut= melt babe, ein banzez gebildet; in sine grofe stugabl you Seiten zerftreut, würoen bieje untitiñslidyen (Erzäly=

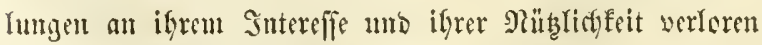
yaben; wereinigt werben fie son ber arabijan Siace cin

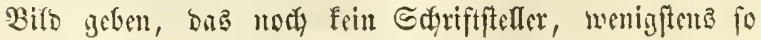

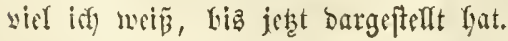

Iith getye zut Garde: 
Den 16. Decmber 1818 fđititen wir ma in Mar= feille nuf ser Bombarbe, Der jungen Clarice, you $\mathrm{Ia}=$ pitän Morgues befefligt, cin. Nm 24. waren wir im

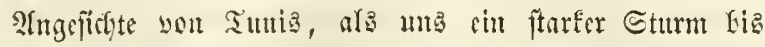
auf sie Söbe won Makon warf. Den 8. Samun 1819 marfen wir auf ber Migebe you Saint = 21ntioes (in Ear=

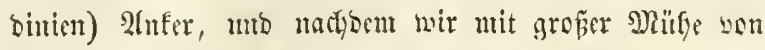

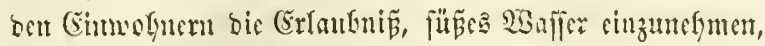
erfalten fyatten, gingen wir beu 10. Diefere Mionatis wie= ber unter Gegel. Drei Tage hatten trir nötgig, um Das

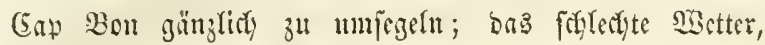

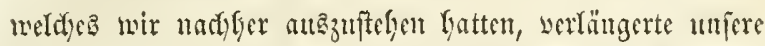

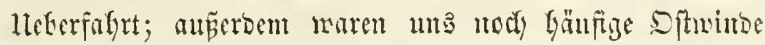
finderticy.

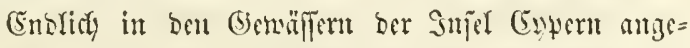

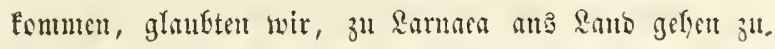

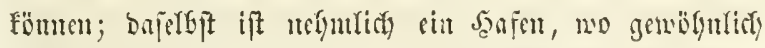

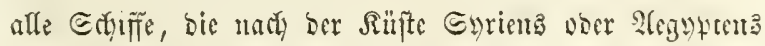

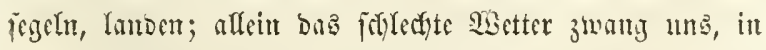

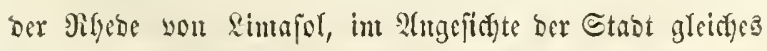

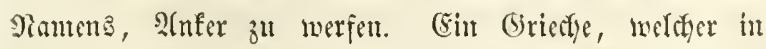
biejer Ctabt bie Befdäfte cintes (5onjuls mefrer Miadyte

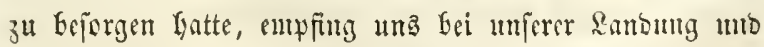
ftelle ung glciti) Darmi bem Mintejellim (Etutthalter) vor. Einige unfedentente Siefryenfe, die wir bicjem mobame= Daniffyen Beamten madyten, werjodanten uns won peiner

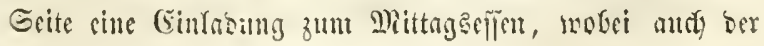


Gonjut mit integrifjen war. Diej̧es Gaftutabl, das crfte,

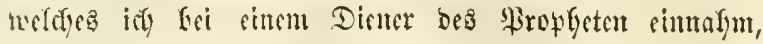

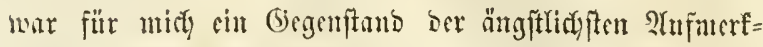

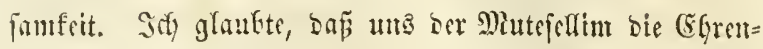
rezeigungen maden müroe, afer bie Safyreszeit, in welder wir uns befanden, war sine Faftenzeit; nir lunglïnbigen

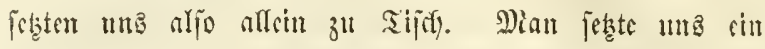
gonzes in feimer Sant gefordes \&amm vor, bas mit Meiz, Binten uno Piftacien von 2fetwo gefüllt war. Der SBcin

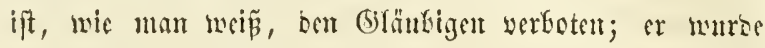
jebod) nidgt gepart, mo berjenige, werdyen man unz reidyte, werlängnete in Nidyts Den forjon fo lange ermor=

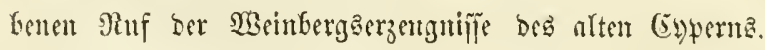
Den zweiten Tang baratif gingen mir wieber unter Seger, unb Ianbeten nod ben 2(fento z"l Rarnaea. SWir giugen

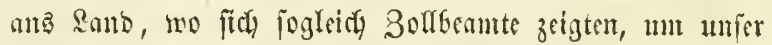

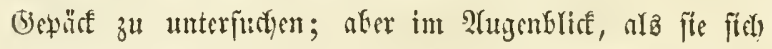

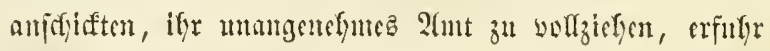

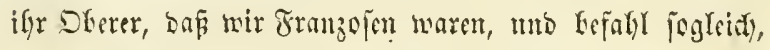

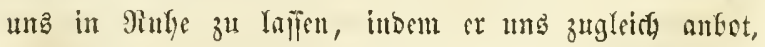
mit ifm in fein Riose (türtifores (Bartentyaus) zu fom

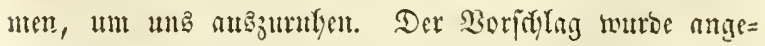
nonutes, und fobald twir bort angefemment waren,

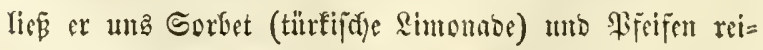

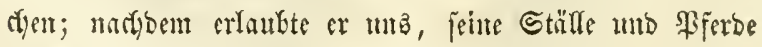

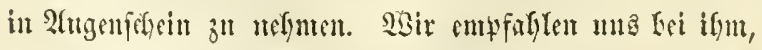

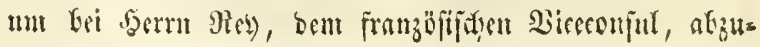




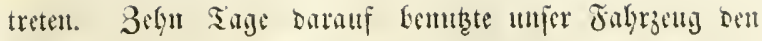
giüfigen Sisind, un unter Segel zu getyen, uno wir famen cmblict) ben 14. Fibruar bor arerantertte (Gan= Derum) $\mathfrak{a n}$.

2rfermorette Eepteft mur mody in ber Erimecrumb. Gräber, Fuinen und einige einzeln ftebenoe forlectote, bau=

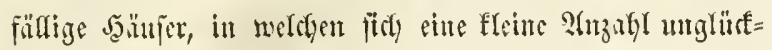

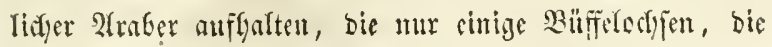
fie in ben Mtoräften ber llmgegento weiden Iaffor, als ifr

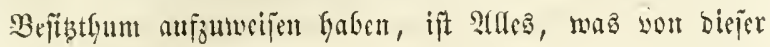
Stabt geflieben ift; fie ift gegemwartig weiter uthts, alz ein malyrfafter Rirdyof, uno un fo meniger bewofubar,

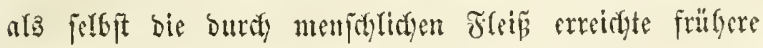
mannidyfadye Bobentenubung ourdy ungefenere Săficr=

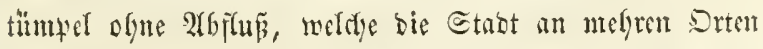

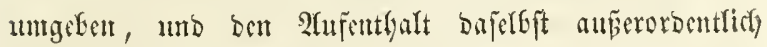
ungejumb maden, verloren gejangen ift. Sluch baften fick Vicr die Eurchäer am Tage wäfrend bor 3cit bes

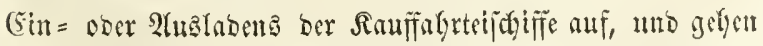

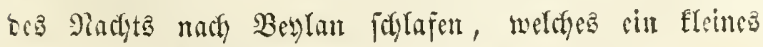
Dorf ift, bas anthitbeatralifa auf cimem ungefäly zmei Stunden von 2flexandrette entfernten Berge liegt, ber

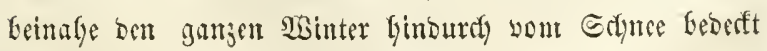
wiro.

Ilnjere erite Corge war, als wir umb z" Illexall=

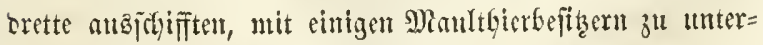

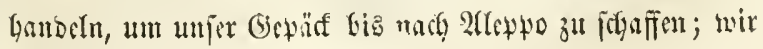


idjliefen barauf zu Beylan, und fegaben uns ben andern Tag anf Den sieg. Bor Arlem nufpten nir ben Berg erflimmen, an meldfen firt) bas Dorf lefont. WSie groj war unjer (Erifinunen, als mir anf bem (5)ipfel anfomen

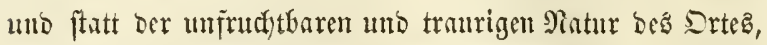

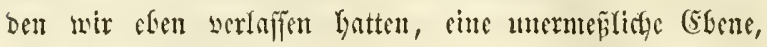
Die Den ladjentiten uno fundytbariten 2Gublif barbot, wor

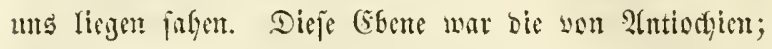

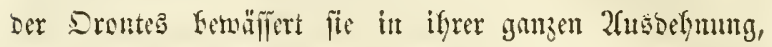
unb überall, mofin mir unjere Blife membeten, fafyen wir mu in woder 2 lütfe ftefoenbe Bänme, nuter ocnen

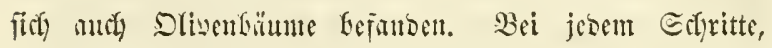

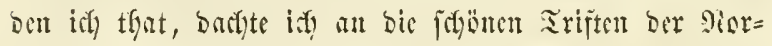

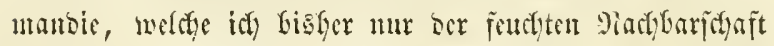

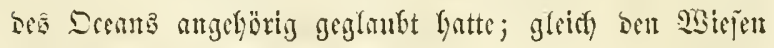
biejer foünen und reifyen Firowing war anth fict bas

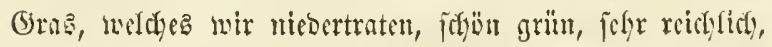

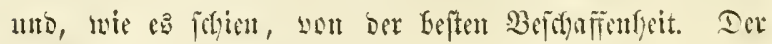

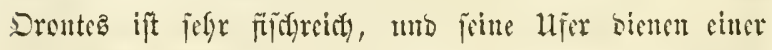

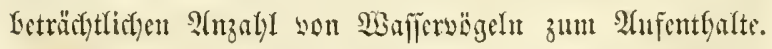

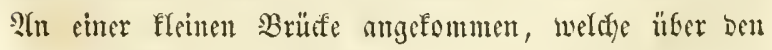
Slup gejolalagen ift, bemerften wir suf bem entgegenge=

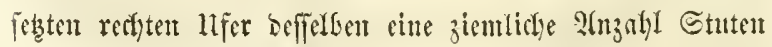

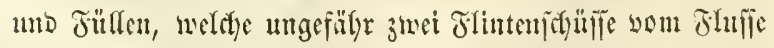

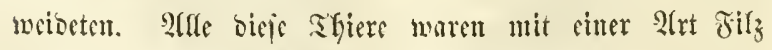
bebefft, ben bie 2truber sifeet nennen, unb ber beftimmet ift, biejalben wor bem Etidye ber Fliegen z"t vermafyrm, 


\section{7}

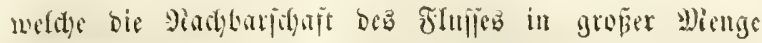

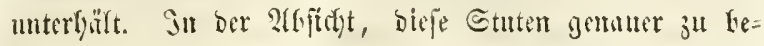
tracten, fegafen wir uns, berr vou Fortes und it),

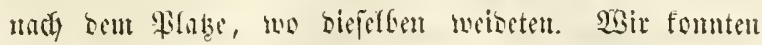
uns benjelfen neit mebr mähern, als wir geglanft batten,

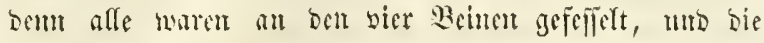

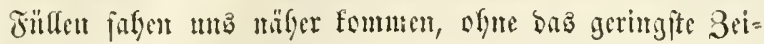
d)en you llurufe ju geten. Dab Saar biejer Pieroe mar

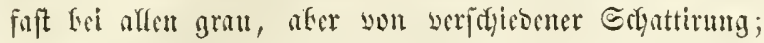

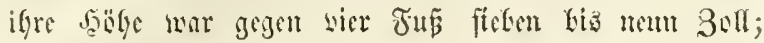

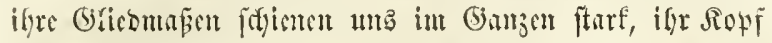
war etwas biff unb mit Greiten Gamajdyen werjefyen; ber

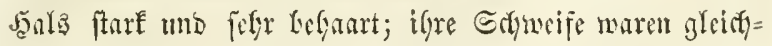

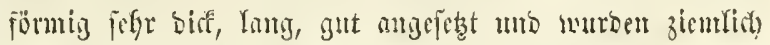
bod) getragen, wegleidy bie Bemegungen berjeleen bei nei=

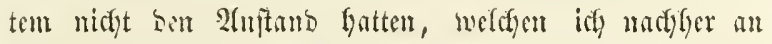

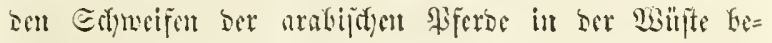
merft gabe.

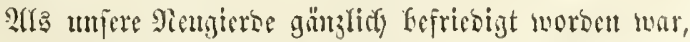
barjten wir batan, urjere fleite Saramane wiebor cingu= folen. Raum ryatten wir aber cinige Esfritte getgan,

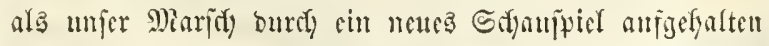

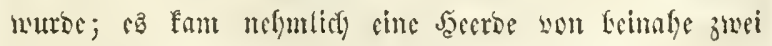
Gunbert Gajellen won cimem föngel fyerab, ber fiti anj ber eimen Geite ber Etraje jamft erfebt; als fite fid auf

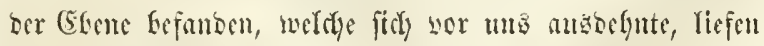

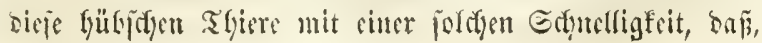




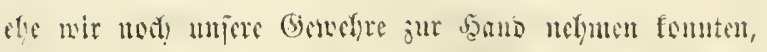

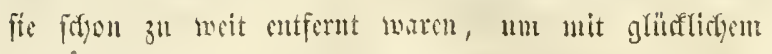

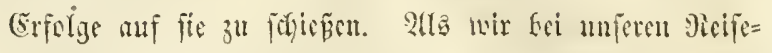
gefäbrten angefoumen waren, ging idf) verjunfen in (5) $c=$

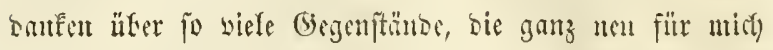
nuren, wor midy biu, als id) auj bem (Sipfer cines gel=

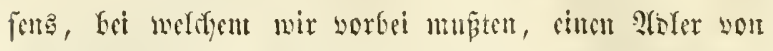

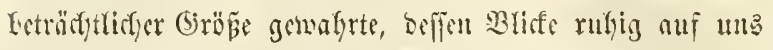

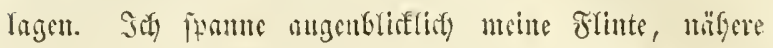

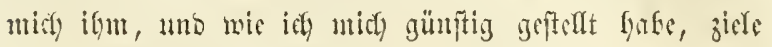

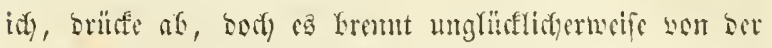

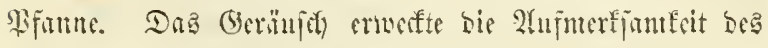

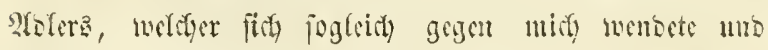

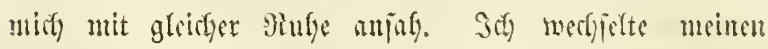

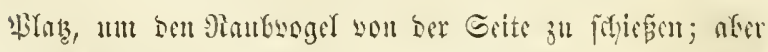
mein Fulver jünocte wie das erfte Mal nirft. Fer Wroler

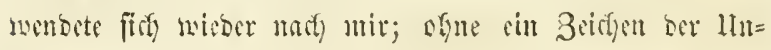
rube, wie vorber, jafy or midy beu Etcit abjoflngen, unfefïmmert un Das, nas idy that; enolidy forthte idy

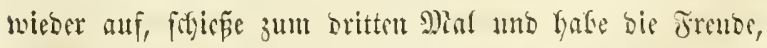
meincn Gegutr mf bie antore Seite bos Feliens ftürzen zu fefgett. Sef glaubte, ign getöbtet zu habeu; aber moem

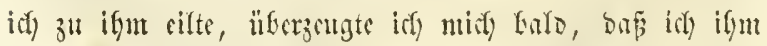
mur cinen Flïgel gelïbunt gatti. Sin zweiter Samwi wurbe aljo nothmendig. Gobalo mirt) ber Roler temerfte,

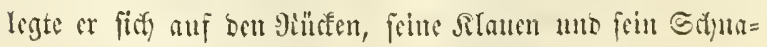

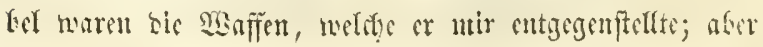




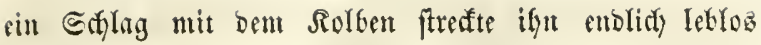

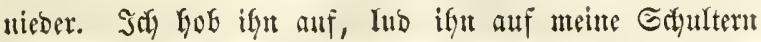
und ging nit meiner $\mathfrak{B}_{\text {eute }}$ im Trumnth zu meinen Mei=

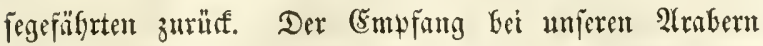
benalym mir aber bie Frettbe üfer meinen Sieg; bem

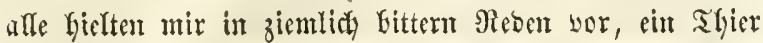
getöbtet zu haben, $D a z$ mir nidat baß geringite Reib zu=

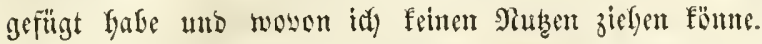
(5)

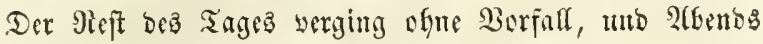

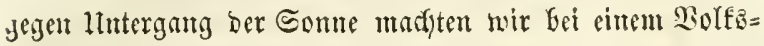

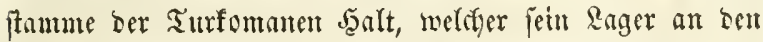

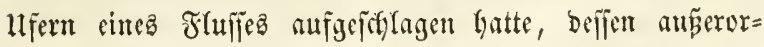
bentlidy flarer Strom won cinigen jeftr reidfliden Sueflen genäfnt twirø.

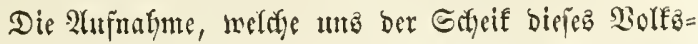

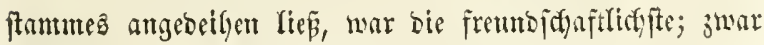
verbanften wir bieferte mohl vorghiglich ber Begenmart

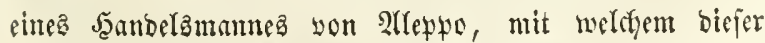

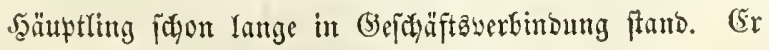
fülyrte uns unter fein Belt, unb nadjocm wir olyne andere

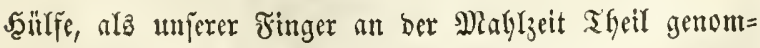
men hattem, entipann fid bie Unterfyaltung; Dant jet

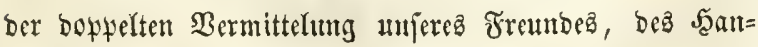

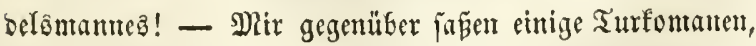

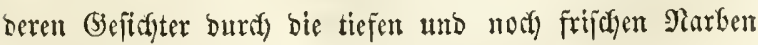

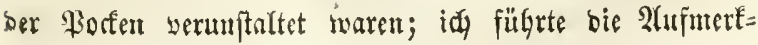


Famfeit bes Gayeifs auf diejen Siegenftand, und fragte ínn, ob er norfy nie won ben in Ëuropa angetwendeten Mitteln gefört Gätte, um biefes ßocfengift ummitkjam zu madyen

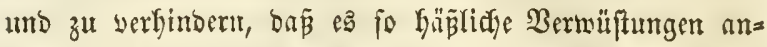
riffte. Er antwortete mit Nein, uno febte hinju, baß Diefe Sranfleit jebes Safr in Rante eine fefr grofe 2 (n= zabl won Spfern forberte. Soth erzälylte ifm mun fogleirh you Den Sulpoden und beren wolyttyätigen SBirfungen, unb fallug ifgu vor, bifielben ben folgenten Ting an einem

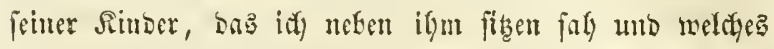

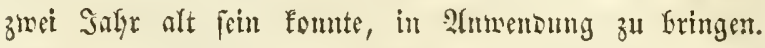

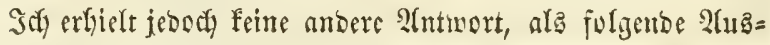
regung eincs ber worzinglidyften Gebote ber mufamedani= jeyen Meligion:

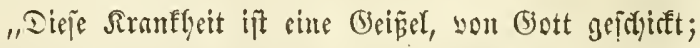

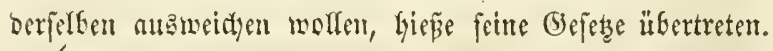

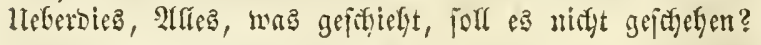

Diejes flelyt gejdyrieben!"

Demnody hiclt id midf nidgt für gejdylagen. Durdy

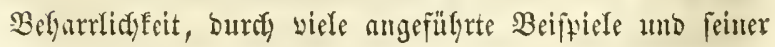

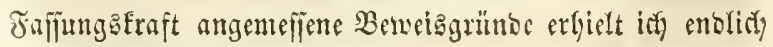
won igm sie Bewolfmädytigung, un bic idy anjudyte.

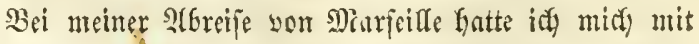

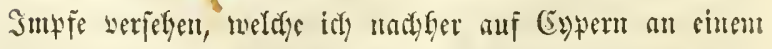

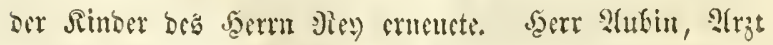

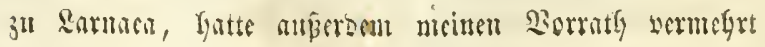




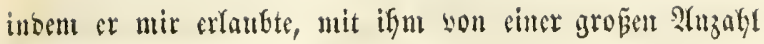
Sinder, bie or eben geimpfrt hatte, afl wir anf ber Snfel antamen, Smlfe zu fammeln. Den andorn Tag aljomurbe mir ber Sofn des Edyeifz anvertraut. Die Dpe= ration unternafm id in Gegenwart faft aller Turfomanen Dez Etaumes. Saum hatte ich biefelfe becubet, ala ich

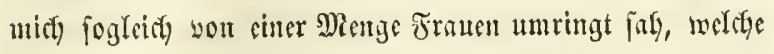

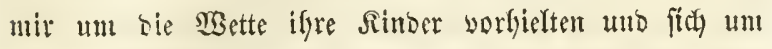
mcine 2hujmerffamfeit utto Eorgfalt ftritten. Eafort be-

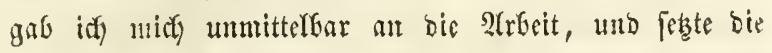
J̧andyabung meiner Rancette nur bam aua, als mir nur

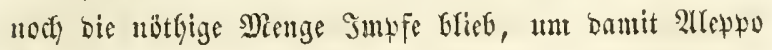
3t erreictyer.

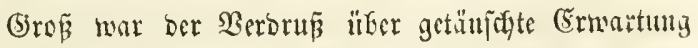
bei bent Mïttern Derjentigen Sinber, weldye idg nifft ofe = riren founte; allein idy minderte benfertben, indem idh

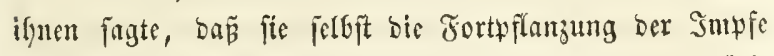

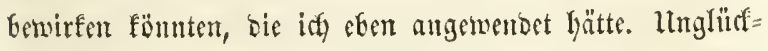
lidjerweife zeigte fien cinc Edymierigfeit, nelymlidy bie Sin=

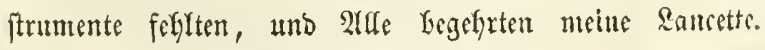
Seider war biejenige, weldye ides in bebrauch fatte, bie

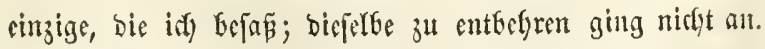

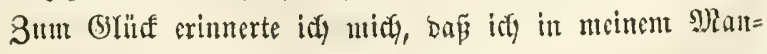

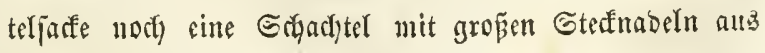
ben Pianufacturen zu Rlelen (Aigle) hatte; iif fonnte jeber

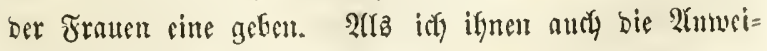
jung gegetent, fidy berferfen zu teshienen, und bic ?(rt und 


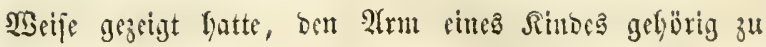

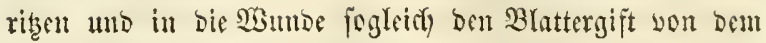

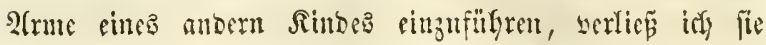
mit ben beften Slünfmünfetyen, uno begab nich mit bem Neft ber Sarnmane auf Den SBeg. Die Etrafie, nelduer wir folyten, nachom mir bicfen Etamm verlaffen hatten,

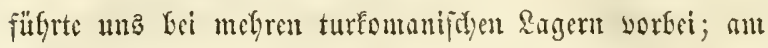

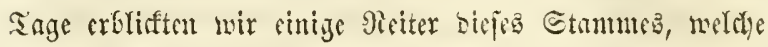
jefre fdöne Siferbe ritten, andy folden wir jiemlidy grope Scerden Gazellen; gegen $2(b e n d$ fauten wir an Juje des

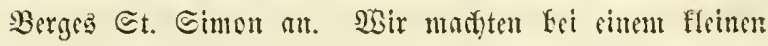

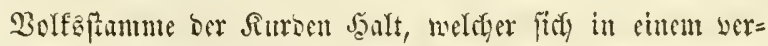
fallenen Dorfe gelagert befans. Diefer Stanm Gejdiä-

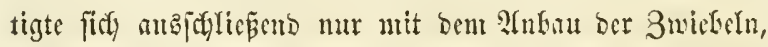
des Tabafz und einizer Gräjer. Der Boben, ben er

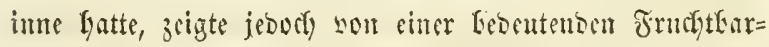

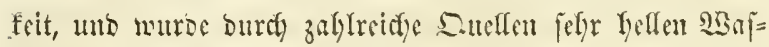

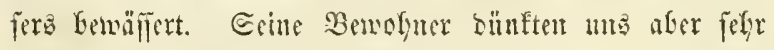
arm zu fein, benn ifgr ganzes Eigentlum forien mur in einigen (Sfaln und ciner flemen 2(nzabl Gityanfon ju be=

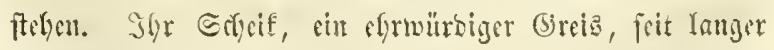

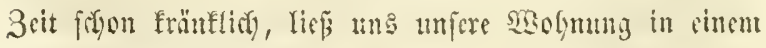
alten verfallemen Gemäuer anmeipen, meldyes fidd neben

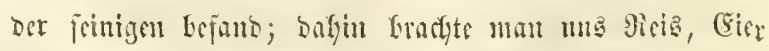

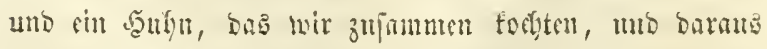

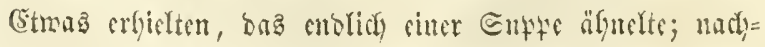

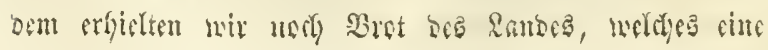




\section{3}

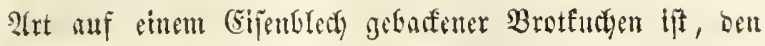

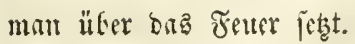

Den folgenden Taj begaben wir unz, narfibem nir bie Buvorfommenteit bes alten Sefeifs unit sinigen Pin=

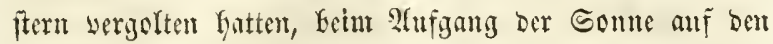

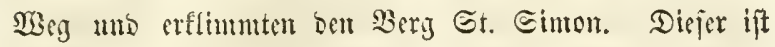

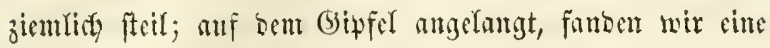
ungeffenere Mlenge હ(f)utt, ia befien Mitte noch cine jient=

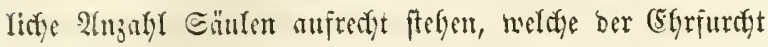

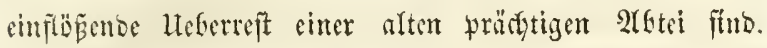

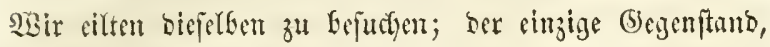
meldfer noch cinige Equren von Ěrgaltung barbietet, ift ein neiter Ninum, wo man über einander in ber Diffe ber

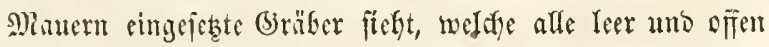
finto. Dieje Riuinen find gänzlide obe unb bienen mur

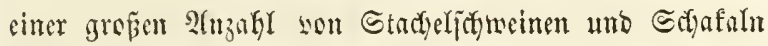

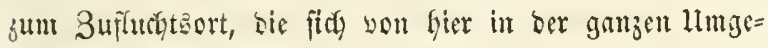
Jetto wertreiten.

Bon ben erfabenen Sunfte, auf weldyem wir uns

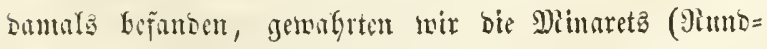

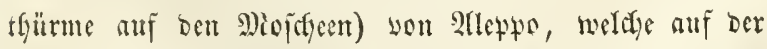

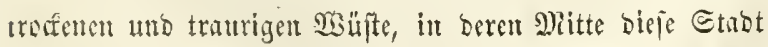
erbaut ift, wie fime Nabeljpisen erjociner. Diejer $\mathfrak{A} \mathfrak{n}=$

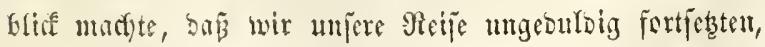

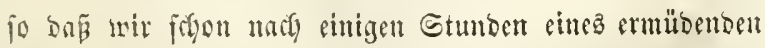

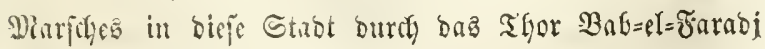
sinzogent. Slidjt weit waren wir im Sumern ber Gtabt 


\section{4}

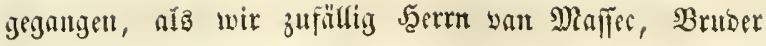

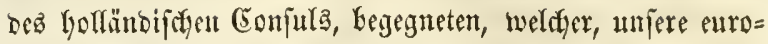

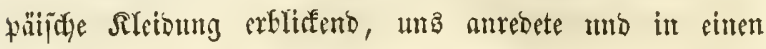

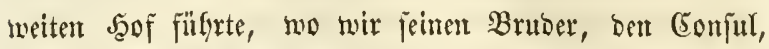
uno ben Grafen $\Re_{3}$ emoutisft trajen. Rebterer if eit pol=

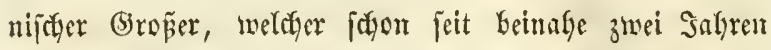

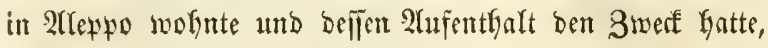

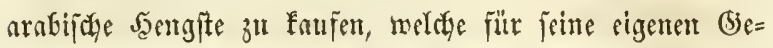

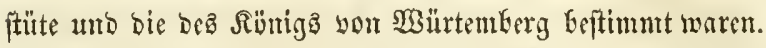

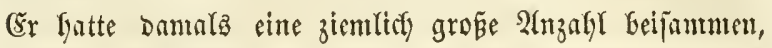
bie er fitch becilte uns zlt zcigem, und weldye efen nact)

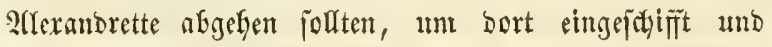

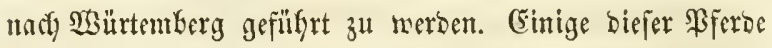

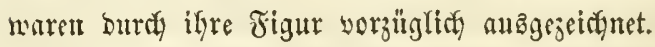

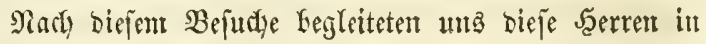

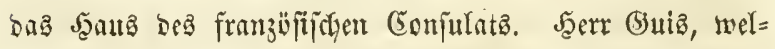

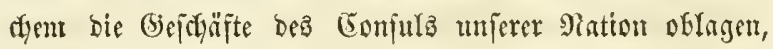
erwartete uns jogen längft, nuld fandent twir bet iffm bie

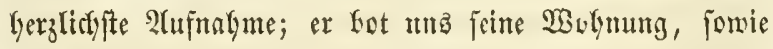
feine Tafel au, Dinge, weldye nir auf bie 3rit amaf $=$

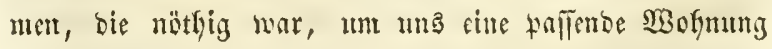

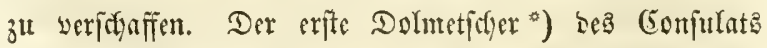

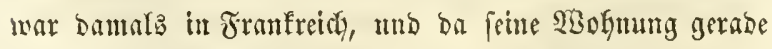

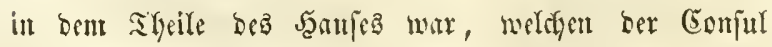

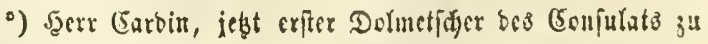
Mleranbrien. 
Eemofyute, fo befant fie fid Yeer, und wir Eebienten umb beriertbert.

In biefer Nadtbaridafaft fonten wir ung mur wobl

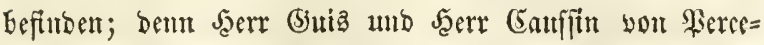

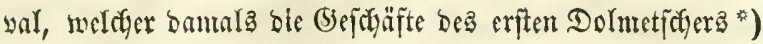
3แ bejergen hatte, erwicjen utta alle mur erbenfliden be= fälligfetten. Beibe Gezeigten fitch ung Geftünbig als anf $=$

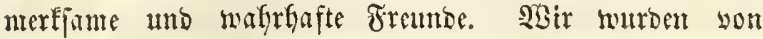

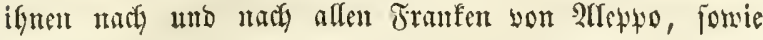
alfen Conjultu vorgefteflt. Dieje Bejuttye madjten wir ge=

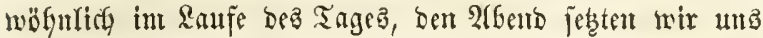

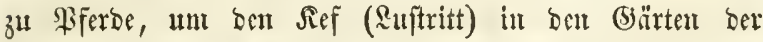

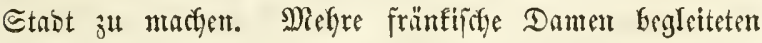
mas, aber nur indem fie ifgr befitsyt nit langen leinenen

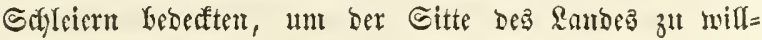
faffren, weldye, wie man weí̈, ben Franen yerbietet,

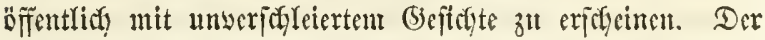

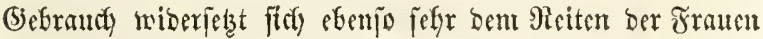

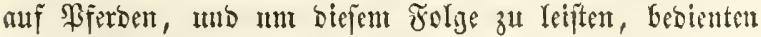
fiid) bie unjerigen ber (šjel. Ěnte einzige üfertrat dịt

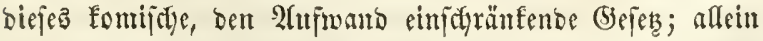

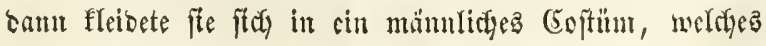

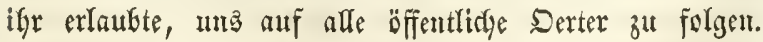

-) Sुerr Camfïr yon \$ercebal ift jesct Profefior ber arabi= fifen Epradje an ber Sobule ber morgentänifinen Spraden.

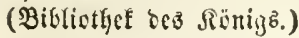


So geĩctyat religiöfen Werfammlung beimofnte, wo cinige beriffynte

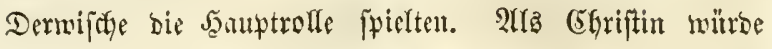
fie unbebingt bayon ausigejdylofien gewefen fein, wäre fie gleid) in unburdyoringliclye Edyleier gefüflt gewejen; aber

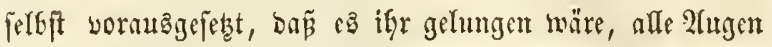

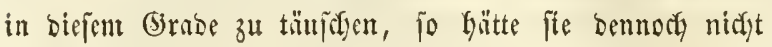
fei uns bleifen fömen, ta fie firit) gejmungen gefertent

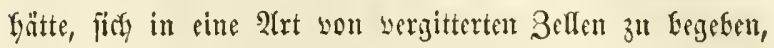

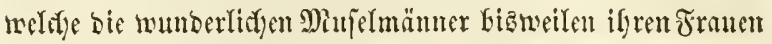
in Den äı

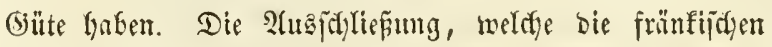

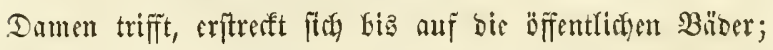
Der Eintritt in biejelben iff ifynen werboten, obgleich ber

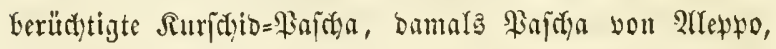

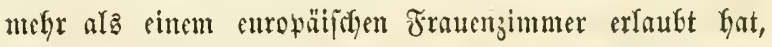
in bas Snutere feines Serails zu bringen und barin bie gefyeimften Tyeile zu bejuctyen.

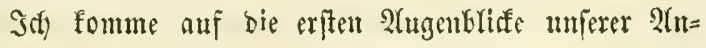

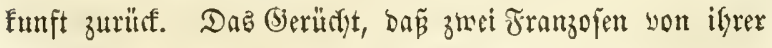
Megiermig beauftragt morben, zu Beffrälem tauglidye ara=

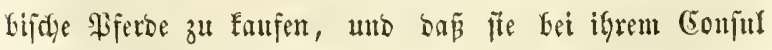
abgeptiegen wären, werbreitete ficty in ber gamzen Stabt.

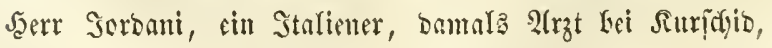

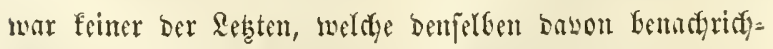

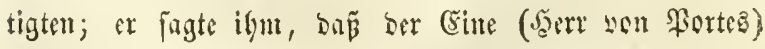
ein (Enir = Iffgor (DFeritalfmeifter) uno ber 2futbere eim 


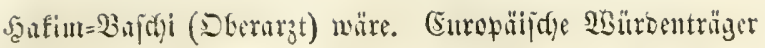

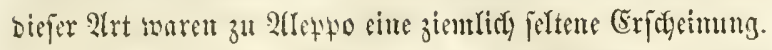

Den folgenden Tag liép nuir aud ber Fajdya burdy

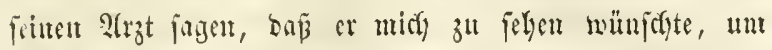
fich) wit unir itber feine Bferbe zu unterlyalten, und mich

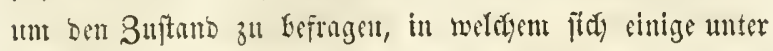
ignen Gefürtben.

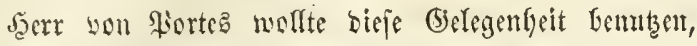

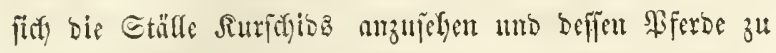

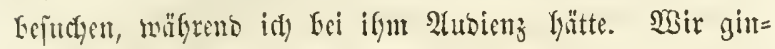

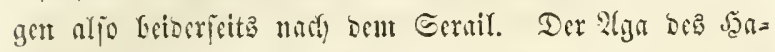
rem empFing uns mit ber $\mathfrak{B}$ itte, Gei iffur einzuttretent, uns

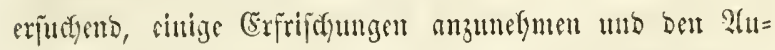

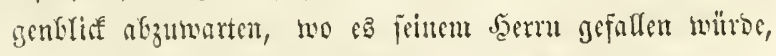
utidy wor fith foumen zu Iafiest. Der Difizier, weldyer

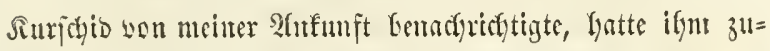

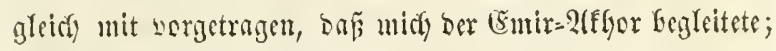

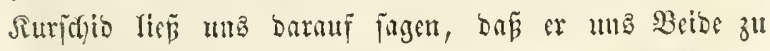

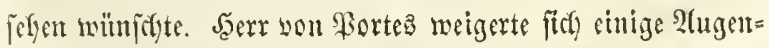
Glife, illoent or bem DFfijier, fo mie bent 2 hag benterffer

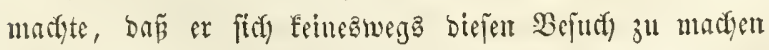
vorgenonumen bätte, or wäre baher mux im cinfacten

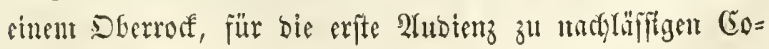
ftüne erichienert.

Eeine Bebenflichfeitent murben bem \$ajffa nitge= theift, welcher nutu um fo briagenber Darauf beftand, in= bem er äuferte: Die 2trt uno ber Siffnitt Der Rleiber 
wathe cine Gadye, bie ign nityt im Seringiten ftöre, nno

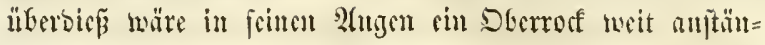
biger als sin Fraff, oa er feineut ferworragenben Thail

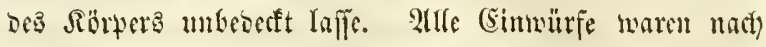

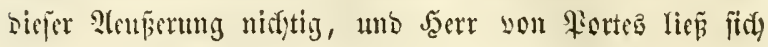

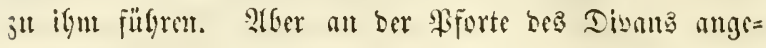

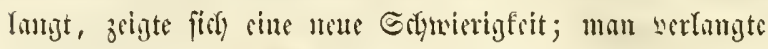

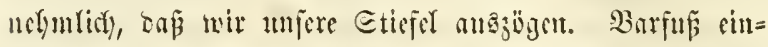
zutreten, founte osn Tïrfen oser Qtrabern ganz notïrlios erfifeinen, weldye mur Pantoffelu ober Sanbulen afuzlegen Gabon: aber Eticfel! (E⿱ erfolgte baljer you unjerer Eeite,

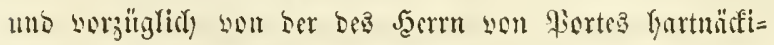

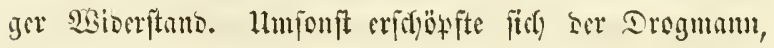

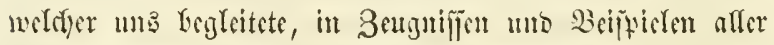

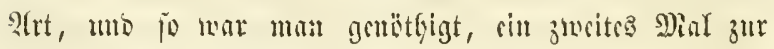

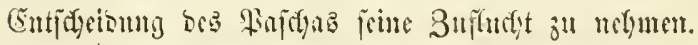

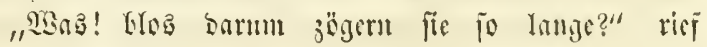

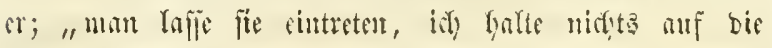
Etifette!"

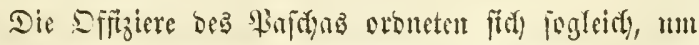
แ⿰氵

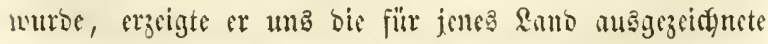

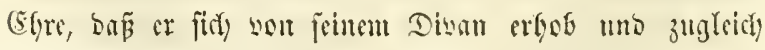

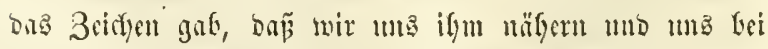

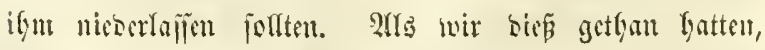

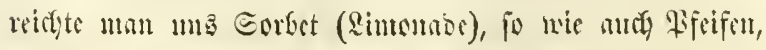

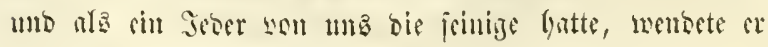




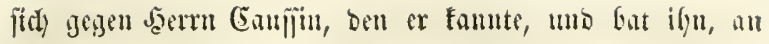
uns eimige Jragen z" ridften ").

Die erfte bejog fich auf Nituoleon; als wir ifm in menig şorten den Etur feimez Saijerreidy mo feine

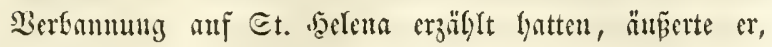

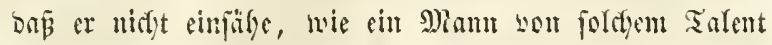

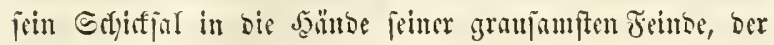

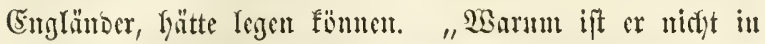

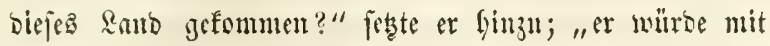

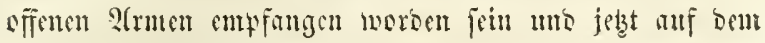

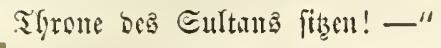

Sm Raufe ber linterfyaltung jagte er mir, baz or fo glüsflicy gewefon wäre, bicjen auperorbentlictyen $\mathfrak{N a m}$ in

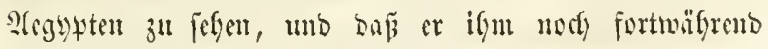

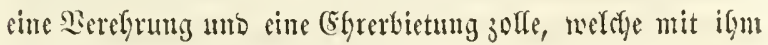
follt alle Drientalen theiltert.

Tie Bujummenfunft bruerte cine Etunbs, weldye er,

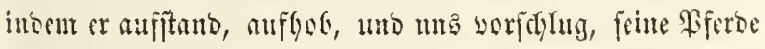

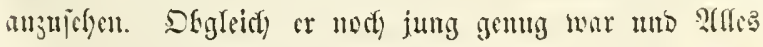

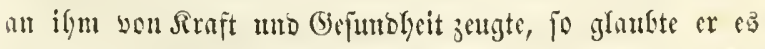

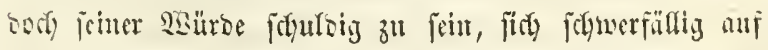

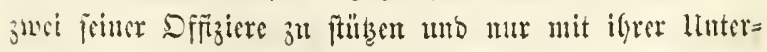

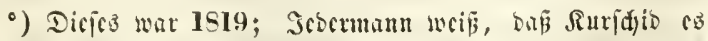

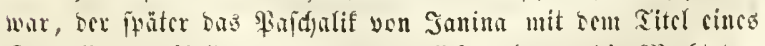

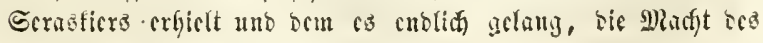

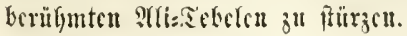




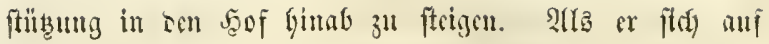
cinen bier befindsictyen 2huftritt geftellt yatte, lies er ung

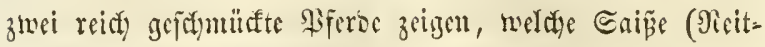
Furctyte) am 3aume ferumfüfrten. (Er theilte unz mit,

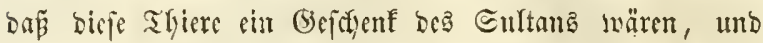

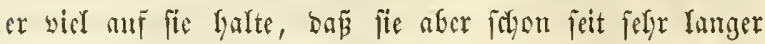
3cit Yaly und anger Etand wären, geritten zu merben,

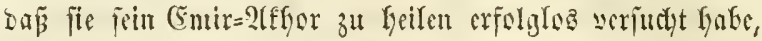

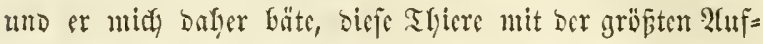

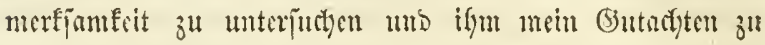
fingen.

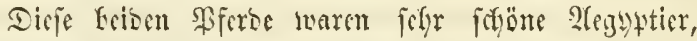
4 ชn 10 3oll fyod, flarf funbamentirt mb ägnefter in Biclem unferen guten Brerben son Merleriult. Esine

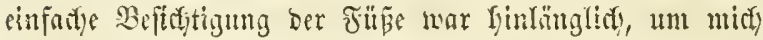
วu

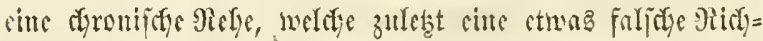

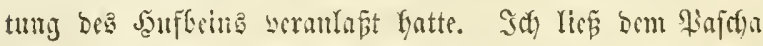

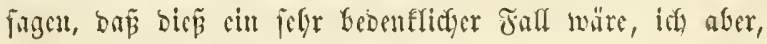
of he einen voflfommenen (Erfolg zut serfipredsen, glaubte,

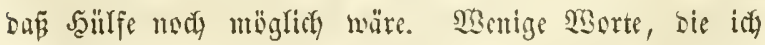
rimzurügte, um meine $\mathfrak{B}$ dyautumg erflärend zu redgtfer= tigen, wurben von ifym fo gut begrifĩen, Dä er jeinen Etallmeifter baran erinmerte, wic bicicr ftandhaft belyautet

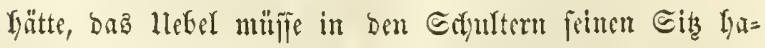
ben, er, als $\mathfrak{x a j a j a , ~ a b e r ~ i m n e r ~ b a r a u f ~ b e f t a n d e n ~ y a ̈ t t e , ~}$

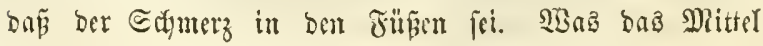




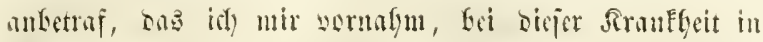

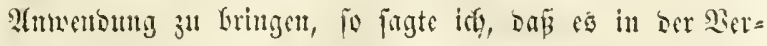

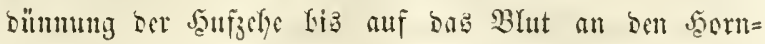

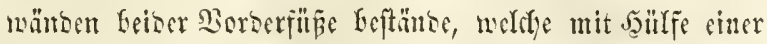

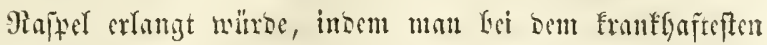

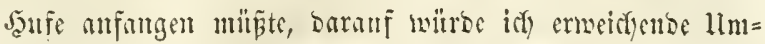

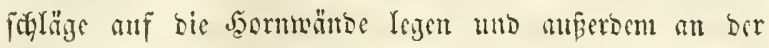

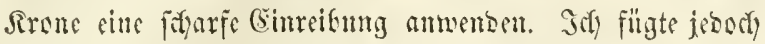

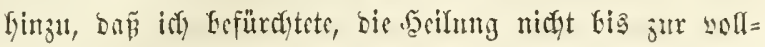
fonmenen Bomejung werfolyen za fönnen, meil wir bie

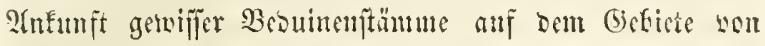
2lleppo crwarteten; id wollte baber un 2 attes in ber

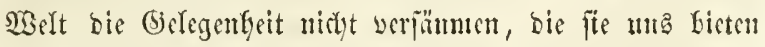

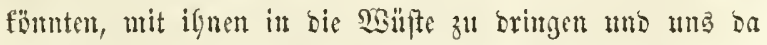

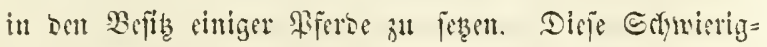

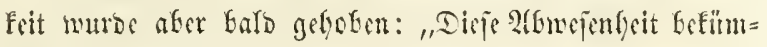
mere Didy nicft," antmortete mir Surjojio; "operire inmure zu, und follteft Du geztumigen feitu, afzureifen, bevor fids Der Errolly wolftünsig gezeigt hat, fo gibfi Da Deine

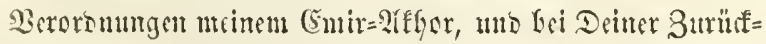

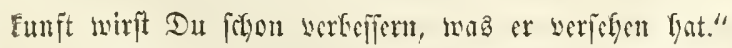

2adyoem bie Speration auf ben folgenoen Tag feft=

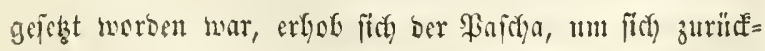

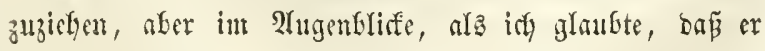

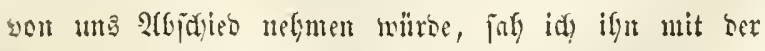

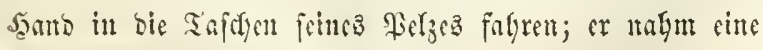

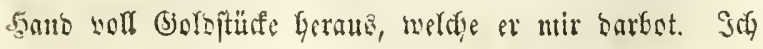


madyte eine ablefuneme Bemegmu, allein wälyrento er bars

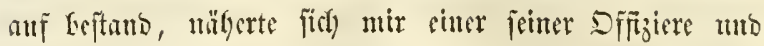

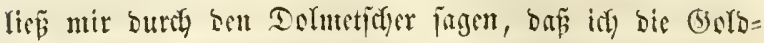

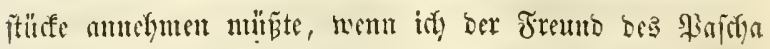

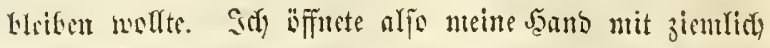

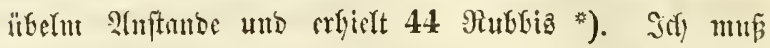

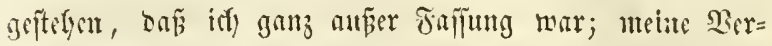
legentyeit sanerte jesody nidyt fo lange, als idg geglantot batte, benn faum wendete mir Sitridyio ben Silifen, als

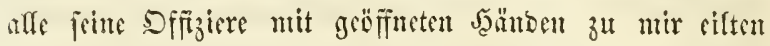

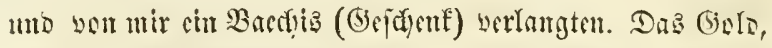
weldies ich eben erbalten batte, reidyte zu diejen gezmun= genen Gifyenfungen nidgt hin, fo Dap id genöthigt mar,

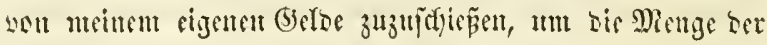
Berlangenson, bie mich einen $\mathfrak{A}$ lugenbliaf unringten, zu= frieben zu ftellen. So wie fite ntity verliep̧en, gingen fie 3u Serrn won Forteb, eine glciclye Freigebigfeit in $\mathfrak{P}_{11}=$ ijructy nelynems, aber sa er nidyto erbalten Gatte, fertigte

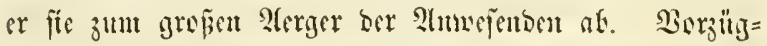

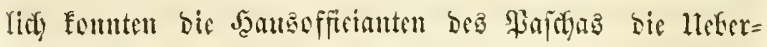
tretung biejes wou dem Bebientenftande aller \$arläpte beg

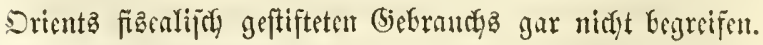
Shre Befdwerben Eamen ofne 3weifel Gis wor Sutridyid; senn ben folgenben Tag fam ber Cmir=2legor, um 5errn you

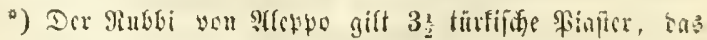
Fitis grgen 2 Jrunfen 50 Erentimen. 


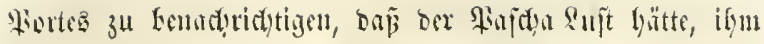

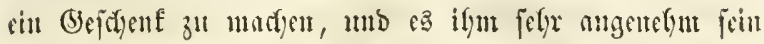

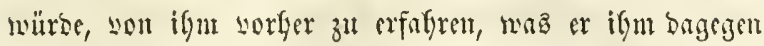

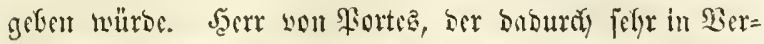
legengeit gerietf), antwortete anf biefe munberliçe (sröfï=

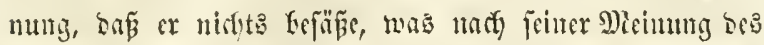

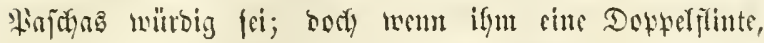
bie er mit nus Franfreidy geforatyt hatee, gefiele, fo würoe

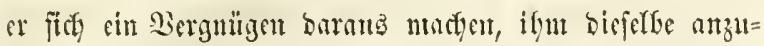
fieten. Die Flinte wurbe pogleirs ben (Smir gebradt, weldyer fie wriffe, fie fefre fodju fand, 1 mo bem Serm

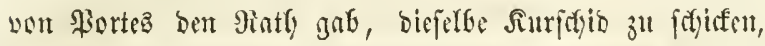

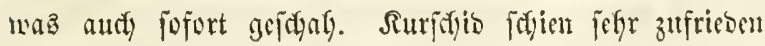
geftellt ju fein, tmb nody benfelfen Tag war Sarr wou

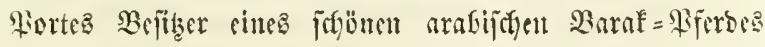

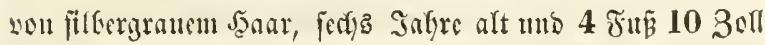

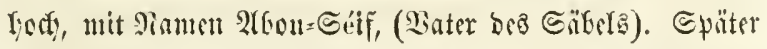

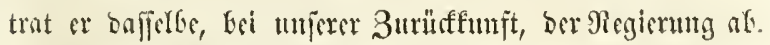

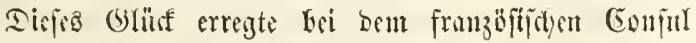

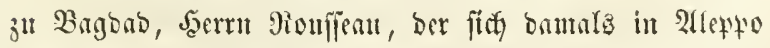

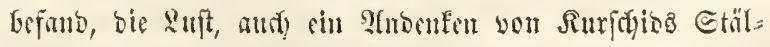

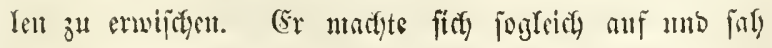

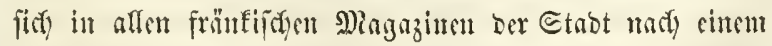
Begenftande unt, ber reidy geung wöre, um ben Faja

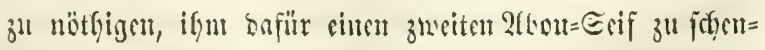

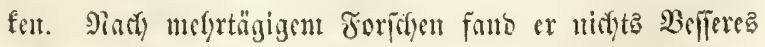

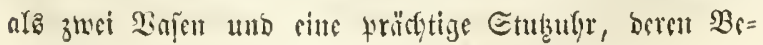




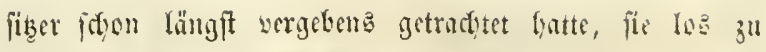

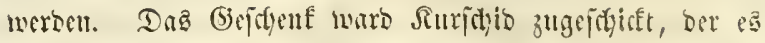

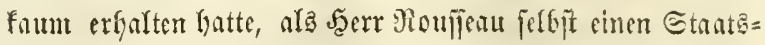
befuch arftattete. Der Fajcha empfing Den Bepuctientoen

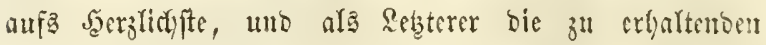
Danfiagungen erwartete, jo nie bic jefurtisfit gemünforte

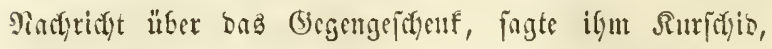
bo

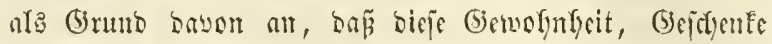

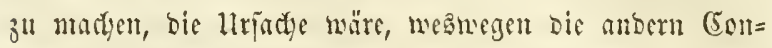
juln serfindert müroen, iffn io oft zul bejucten, wie or

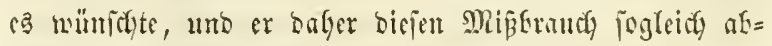
icharfen mollte. Bugleidy gab er ben Beferfl, bie Etuth=

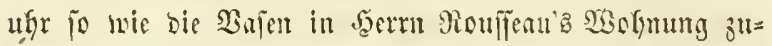
riuff zu bringen, banfte igm für jeinen guten Sistllen, bat

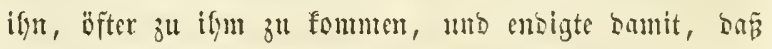

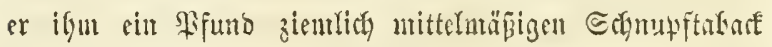
zutommen lię.

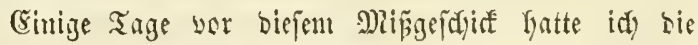
beiben äguptifegen Pferbe operirt, won benen itif obent

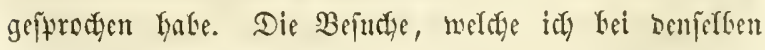

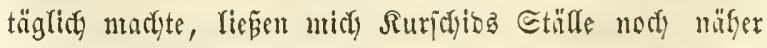

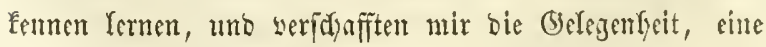

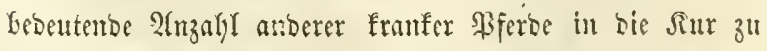
nelfmen. Biele unter ibuen hatten ben :

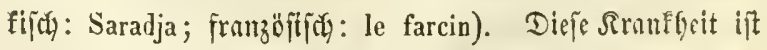

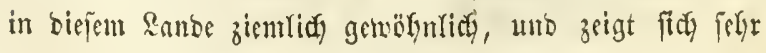


Gartüafig; bie Türfen glauben, Deriflben Lorgubeugen, Doer fie zu befïntpfen, ment fite ausgebrodyen ift, indem fie einige Sdymcime mit in bie Ställe nefmen; auth Surr. fidio Yatte Sorge getragen, cine grope 2tuzabl berfelben

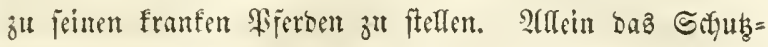
mittel blieb umwirffam, worhber or fict) wumberte. Jith

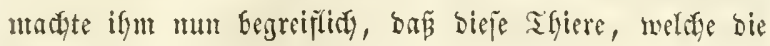
Türfen übrigens für unrein halten, anfitntt bicjes Hebel з̆ vertreifen, baffelfe im Begentyeil Duridy ifyre llnrein= (ict)eit erzeugtert. Ja rieth ifm aljo, feime Gtïlle yor aflen Dingen won biejen Thieren za füubern, unb als biefer erfte Griritt für bie Gijumbleit ber franten Biferbe gethan morben war, nafm idf biejelben in meine Bebano= lung, unb affe muroen joundf gefyeilt.

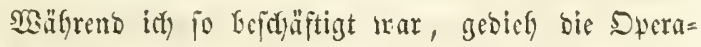
tion, weldye ith an ben Jufen oer beiben ägyptijat)en. Prerbe gemarift batte, gang vortrefflidy. Samm waren zmei Monate vergangen, alz fie Der Bajada wiécer reiten

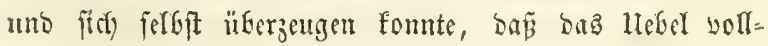
fämbig gefroben mar. Dieje Sur Eradyte mir 500 Pinfter ein, weltye ex mir burrty eitren jeiner Dffỉiere j̈berbringen

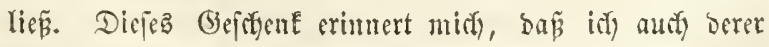
gebenten muE, bie iris, jo läptig fie mir auch maren, jebeäutal bon ifm erfielt, wenn idy ifm begeguete. Samm midy Surjobio auf fcinen Cłajiergüngen gemaly murbe, liés er mict) allental zu fitch rufen, unb gab mir bieferbe

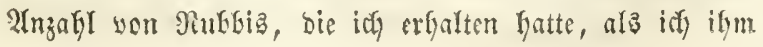




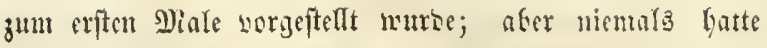
idf 3eit, fite in meine Tajde zul ferfen, benn fogleich fab id) midy wou ciuter Mienge Dfïjieren unto Renten wom

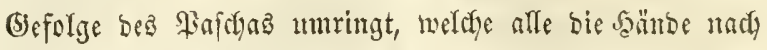
mir auŝftrefften unb mict) nöthigten, unter ifnen alle fiz

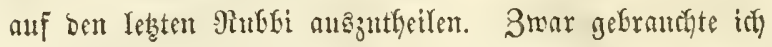

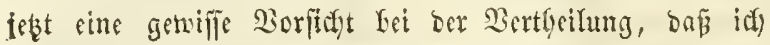
wertigftens nidft melyr nötgig hatte, meine Jreigebigkett anf Soften meines eigenen (5elofecteles ju ergänzent.

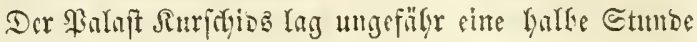
won ben Dianem Der Etadt entfernt, unb war won ber= felfen burdy grope Tobtenilfer geffitedent. Cines Tages, als ith mich quer burch biefe traurigen 230 lynumgen balyin beigab, begleitet won cinem Diener des Rambeb, mit $\mathfrak{N a}=$

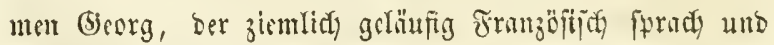

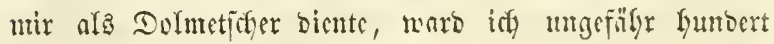
und fünfzig Crfyritte vor ung brei 2(rnauten won ber 5jarbe bes łaqdyas gewafyr, meldue fogleidy forben bliefen,

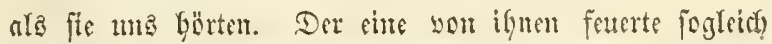
feine grinte nach ber gridytung, in weldyer wir folgten,

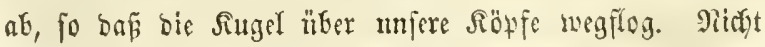

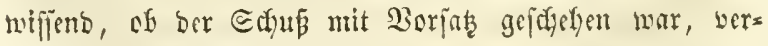

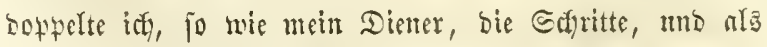
nit bei ben 2 (rnauten angefommen maren, erbob idy meine Etimme und fragte fie, of fie ben Sefung gegen mo

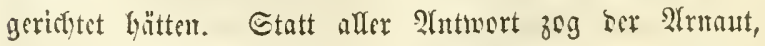

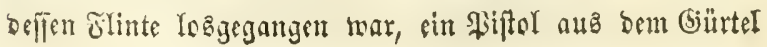




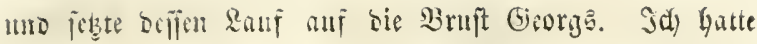

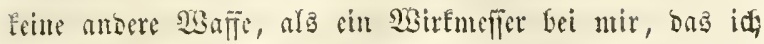
ifgleidy nus bor Sajdye jog, indent inf biejem Elenden

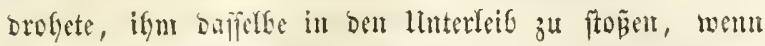

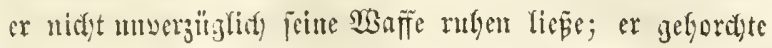
mir, uno cinige Gefunden nadyer gingen feime beisen Rameraben mit ifym meiter. Eogleidy gab iff mesuem Dienter ben Befebl, ifucn zu folgen, ofne fte aus bem

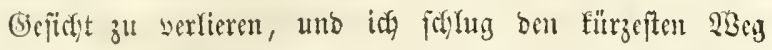

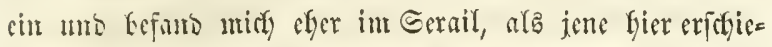

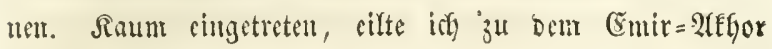
(EGerftafluteifter), uno on idy midy igm nicht verftämblid

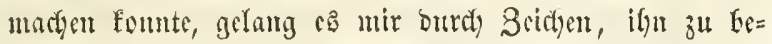

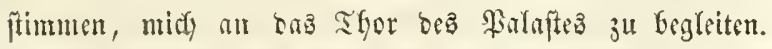

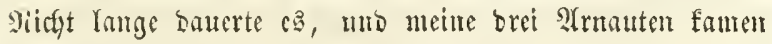
atr. Geory, ber iguen ziemlidy nabe folgte, beeilte fid, mux jogleidy bem (Smir zu erzäblen, welder Gefafyr nir eben entigngen wären. Diejer Rebztere werlor feime

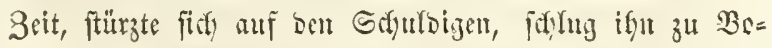
ben und verjebte ifm jo beftige und jaflreiffe @töpe mit

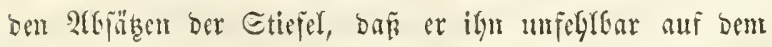
Slabe getöotet Gaben wḧrbe, went ich nicht Ginju ge=

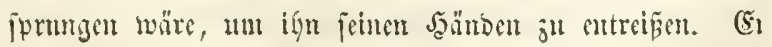

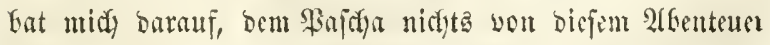
ju jaget, uno gab mix bie Berfidyerung, baz er jogleid

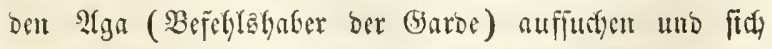
mit ifn befpredyen würse, un ify ju veranlaffen, baf 


\section{9}

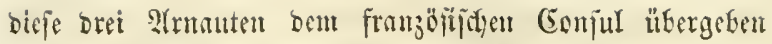

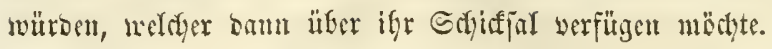

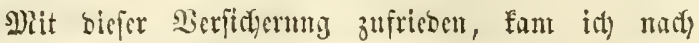
2repto zurüif und erjäglte Serrn won Fortes, fo wie

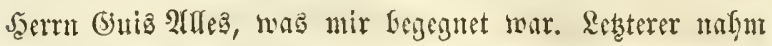
bie sadje fefre ernfthaft unb faute mir, baj, ment ifm bieje Mienfdyen nidyt form ben morgenton Tag ausgelie= fert mären, jo mïhte idy bein Faja Slage fübren, um

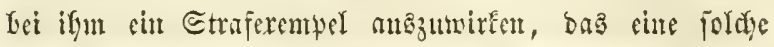

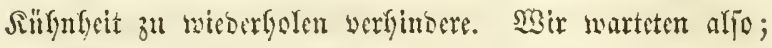
Da aber Riemand erjofien, fo Eafyrte ity ben folgenben Ting gezen fiefen Mhr in Den Cernil zurïf; biejes Mial afer

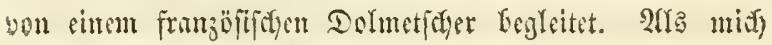
ber अajda im Sofe bemerft Gatte, Yicß er midy jogleidy über ben Grund befragen, ber midy fo zeitig fierfyer fïlyre. Sir) ftefle mici) gerabe wor ifm fin, wäfrent ber Dol= metifyer meinen Dorfall mit ben S(rmanten erjäflte.

"Müroeft Du sic હchurbigen wieber erfenten?" fragte midy Ruridyis.

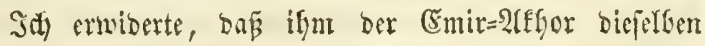

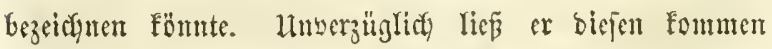

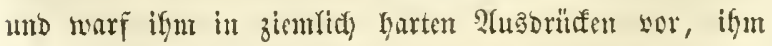
you Diefer Begebenbeit nidgta gejagt ju Gaben; Der (Sntir

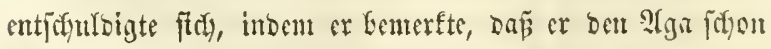
bayou unterridytet hätte; biejer Retetere wurbe gleidyfalls gerufet, befanto fiefy aber abmejent.

"Du famtit Dira negbegeben," faste Suridyis zu mir, 


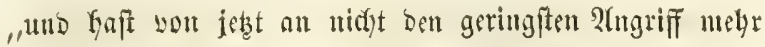

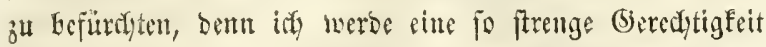

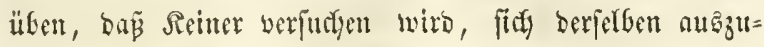
[eţene."

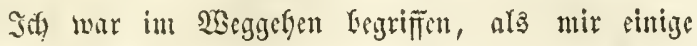

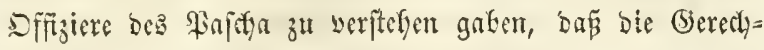

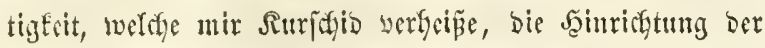

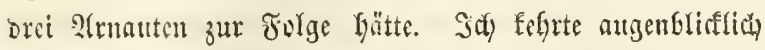

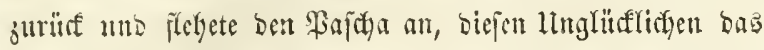

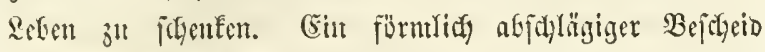
war bie 2hrtwort. 2lber auf vielea infitünoiges $\mathfrak{B}$ itteı

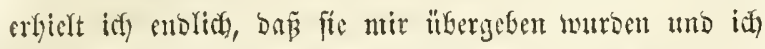
cine joldte Gtraje über fie werfängen Eonnte, bie mir taj=

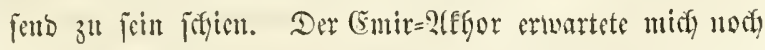

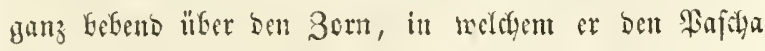
fecchent Gatte, an ber \$forte Des Diwans; er füfrte midy

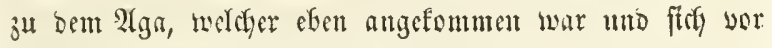

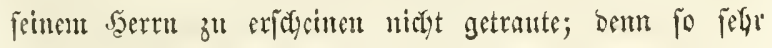

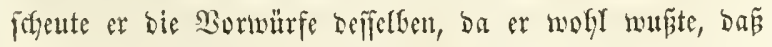
fie tha treffen würoen. Betoe verlangten meine Befefle;

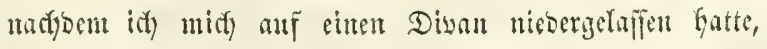
morauf ich $\mathfrak{B c i b e}$ fith zu fetzen nötfigte, Gefaffl ids, mir

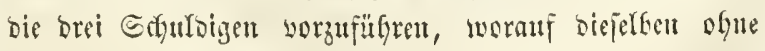

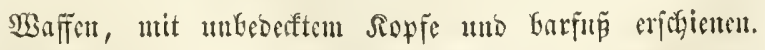
S3äfgrento man fie holte, hatte ber Ěnir jo wie ber 2 (ga

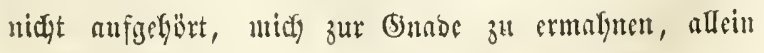

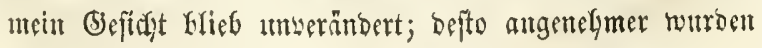




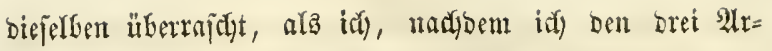

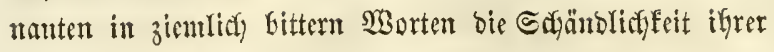
Sanblung vorgebalten batte, mit ber Creflärung ber unbe=

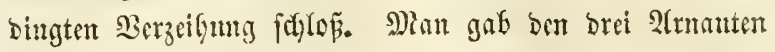
fogleidy ifre Waffen wieber, uno ity ging burch zmei $\Re$ iei=

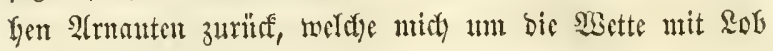

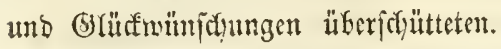

Die Gmabe, won ber idf cben ben Bemeib gegeben batte, mar jo jelten naty den Gitten bes Rambes, daṕ bie brei Arnaten gar nitft an ifre SSirflichfeit glauben

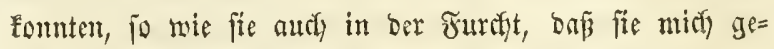
reuen mörfte, in ber folgenben Racht Dawon liefen.

ซেaft zu beriflesen Beit madyten wir unjeren erften 2nfaur. Geitben man in sande in Erfafrung gebracht

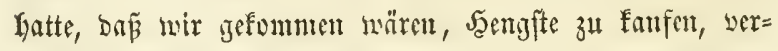
ging fein Tag, bap̃ nan mus nidyt einige Derielben vor= fïfrte. 3u ben 24nerbietungen, weldye man ma madyte,

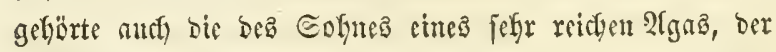
bie 2tomejentyeit frimes Saters, weldyer fith auf ber $\mathfrak{W a l l}=$ fayrt nacis Micfia befants, bemuste, uns nady und nacty alle Bfferde feiner Stülle zu zeigen. Cே̈in cinziges unter benjelben fdien fïr unछ zu pafjen. STir befragten ifn aljo um ben झreib, moranf er 2,400 türfipdye Fiafter (1,800 Franfen) forscrte; diefe Sumnte fojien uns jo felyr unter bem malyren Saertye zu fein, baf wir einige

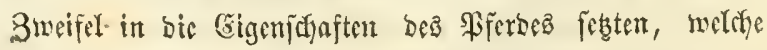
er gerüfunt gatte. gity werlangte bafyer bas gifero zu 
reiten. 3wei Tage Ginter cimander werfudte id baffelbe geförig, mo jebez Mal fand ich in bem Thiere grof́e Sriffte. Şerr you Portes bot bierauf 1,500 Piajter $(1,125$ Trautert); ber Solyu bes 21 ga erbob mit allem Siedft Darüber cinen grop̈en Rärm, aber an (Ende ließ̄ or

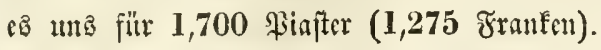

Da biejer Sengfi ber erfte war, den wir fauften, gaben wir ifut beu Nanten Mrgeby) (Metein). Dex Fin=

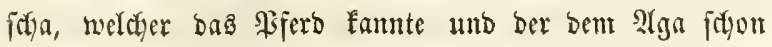
felbjt cine fefr bobe Summe fït baffrlbe geboten hatte, matyte unz

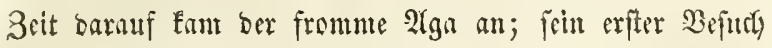
war feinem Stalle gentomet, wie groj mar aber jein

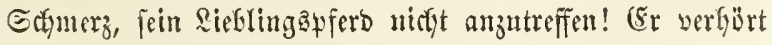
alle feine Rente äber bie Itrjadje you beffen Berifjumben, uno als er erfäbrt, meldye Rolle fein Golyn dabei geppielt

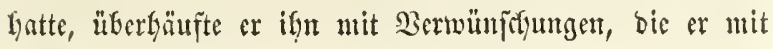
Stocfichlägen begleitete, und Yiẹ zu Surjofio, um von bic= fen die Burüfgabe feine guten Pferdes zu crlangen.

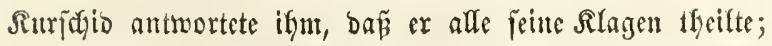

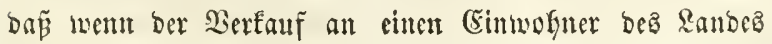

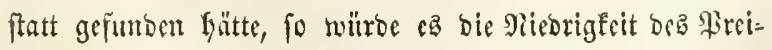
jeछ geftatten, benjelben burch Storfjeläge zu zwingen, ben Sauf z"t Erecten, gegen meldyen er gefommen wäre, Csin= fpruty zu tfun; aber ba bie Säufer Jranen wären, fo fönnte er mur wou bent Mittel ber lteberrebung (Gebranct)

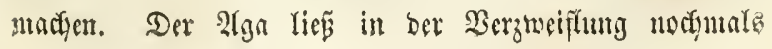




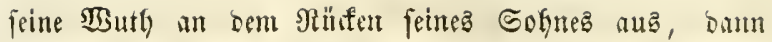

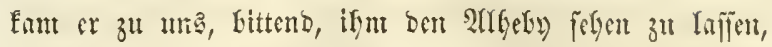

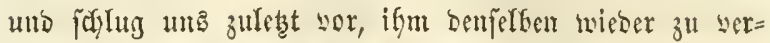
faufen. Da wir aber mit unjerer Frwerbung nut ju jefr jufrieben waren, um bicjelbe mieber aufzugeten, fo wurbe ber Borjalag bes 2lga verworfen. (5r erjudute ung Daranf, cine Etute yon ben J̧engft belegen zu liij=

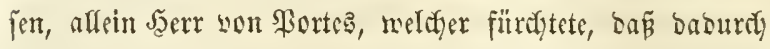

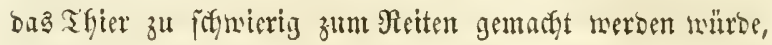
(id)lug $e$ ab.

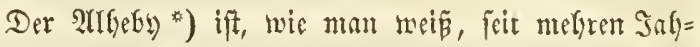
ren einer unjerer beften $\mathfrak{B} e f \mathfrak{d y a ̈ l e r ~ u n o ~ i f t ~ v i e l l e i d y t ~ w o n ~}$ allen biefen ciner ber angenefymften fïr ben Seiter. (5r Gat in Rimoufin sine bebentenbe, ausgejeidurtete Radifom= menidfaft, Deren eine ziemlidy grope Sanjalyl in ben S3ett=

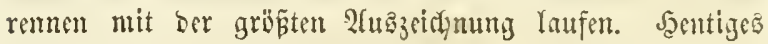
Tages ift or in Geptüte zu \$an.

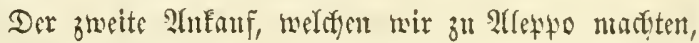
war ein Eraumes einjägriges Füllen, nit શamen Dutfeif

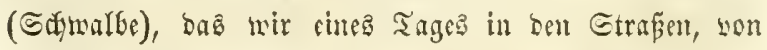

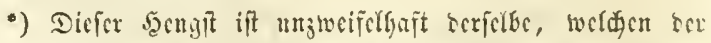

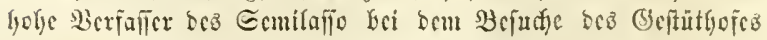

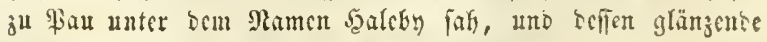
Eigenfogaften er mit ber grëß̄ten Begeifterung rïlymt.

Der lleberjeber. 


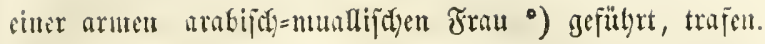
Mrz wir über bent Freis mit ifr üfereingefonment waren,

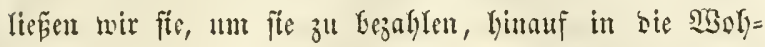

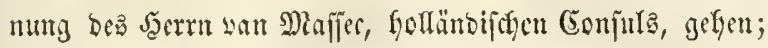
intoem fie mut in bie Sallerie trat unt ifgre Bliffe ber Reife nadi auf alte Familienbiloer fielen, bie an ben

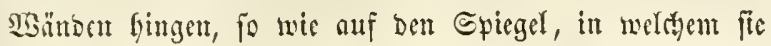

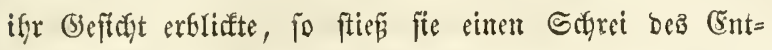

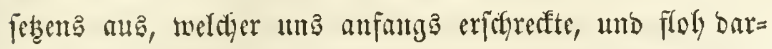
auf in ben Şuf. Shir maren bafer genötfigt, himuter

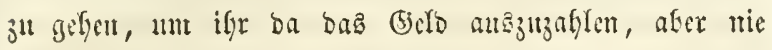
fornten wir von ifyr erlangen, wieber binauf zu gefou,

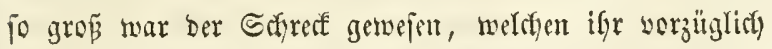
ifre eignes Bilb im Epiegel verurajacht lyatte.

-) Huycucin arme Rlafie yon STabarn, wolefje bic llurgegcud

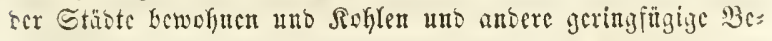
surīiñe घerfisuch. 


\section{Bueites Sawitel.}

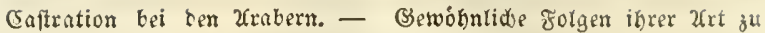
caftriren. - Reife nad Rillib. - Sonterbare Begegnung. -

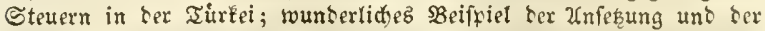

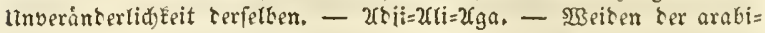
fhen Syferte; von fer frt wie fie angebunten werben. - Segen= feitige Befdente. - Kberglaube ber Turken. - 3auberci; it werbe ferfelben befdultigt. - Eatoverfdau. - Xbreife vou Rillib. -

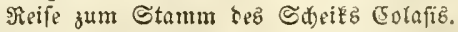

Sn ben erjten Tagen dea Mionaţ Miai 1819 ịchiffte mir ber Mintejellim (Stattbalter) von Rilliz mo

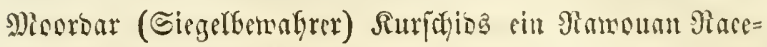

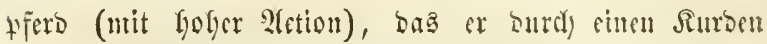
Latte eaftriren lajpu. Dic Eaftration, powie man bicfelbe in biejen Ranbe auBübt, bat oft ben Sierluft Der Thiere,

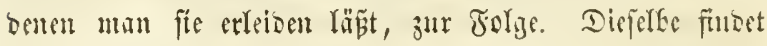

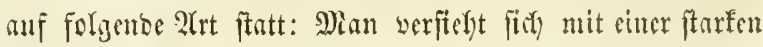
Bange mo ergreift samit bie Sobcu ses Thicres ofer= barb ser giebutboben, barnuf jerquetidyt man fie, shue

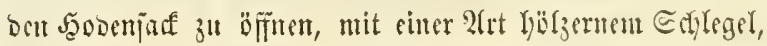
woldyer ben Dient einez Sammers berridtet. Diefe Garbarijo Methode veranlap̈t gewölynlich eine gefäbrlitye Sutjünoung, welde oft ben Brand utb barañ ben Tod

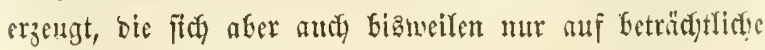
Rerftopfungen im Itnterleife bejofränft.

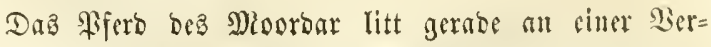
froffung Diejer 2(rt. Narb einer lluterfurtung yon einigen Mimuten fagte idy bem Serm beffelben, sap idy bic Şei= lung nibjt verbürgen fönute, jebody saffelbe wermittelft 


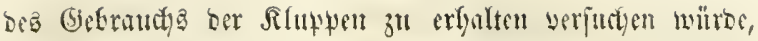
inben idf mit benferfen ebenjo zu verfafyren gebädate, wie

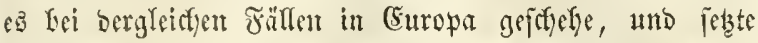

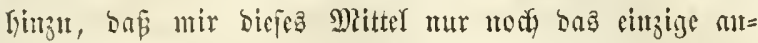
wenbbare zu feit foriene. Sot erbielt alle Gimalt, zut verfalyren, mic ç mir gut bünfte, und oer Sranfe murbe

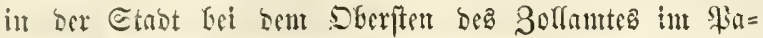
fưalif grlafient.

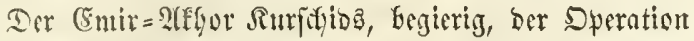
beizumolynen, Gatte nich gebeten, fie im \$oofe ber Ställe

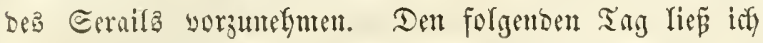
saber ben Sirnfen bei guter Bait babin fülyren, tub mollte mith fo eben an bie Sabeit begeben, als mict)

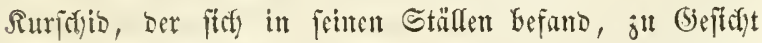

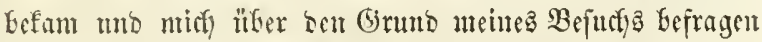

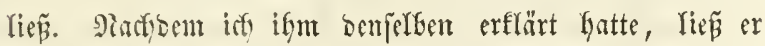

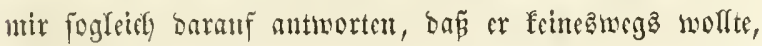

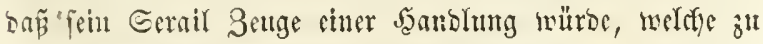
miduts Beringerem füfre, als augenfïllig jum Sweifel au

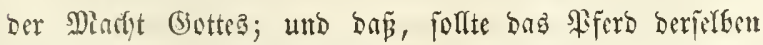
unterliegen, idf mith begnügen mörde, feime Sceilung von

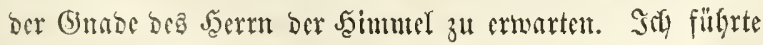

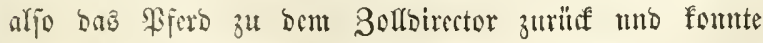
fier entolidy operiren. Siart) vier Tagen fing bie Ëiterung an fidy ju biloen, unb yiergelyn Tage waren nody nifft

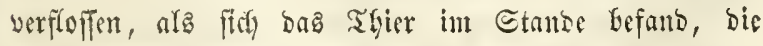
Streffe von zmörf Stumben, olne bis geringfte Gidwie= 
rigfeit, zutrüfłulegen, meld)e 2(letwo won Rillis trennt.

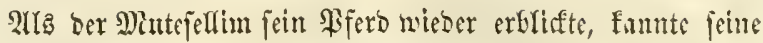
Jreube feine Grenzen: mitroe autf) fidmer fein, weicfere Giangarten, als bie biefes

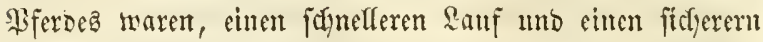

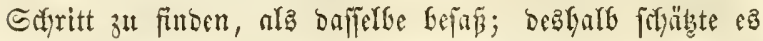
fein berr and aup̃eroroentlidy mto ritt bafieltbe allemal,

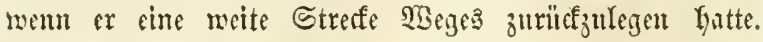

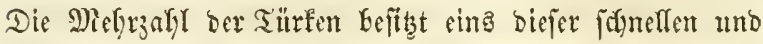
angenefymen $\mathfrak{B j}$ crobe, weldye jëe mit ciner 2 frt yon Saum= fattel reiten mb fie Pamonan=gbifye nemnen.

Cinige Tage nadyber, als mein Sranfer ztt feinem

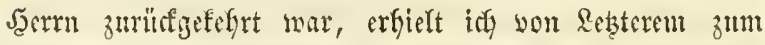
Gejdenf ein in biepen sanbe fefr beliebtes 3utermert,

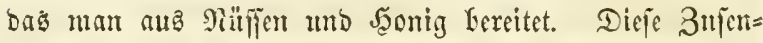
bung war you eitmen Briefe begleitet, in meldyent mid, ber Ěnir = 2lffyor bes Metejellims nard Sillis zu Fommen

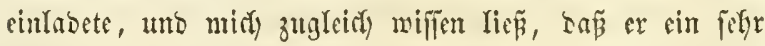

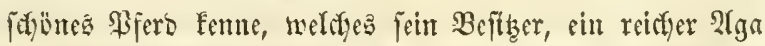

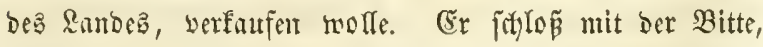

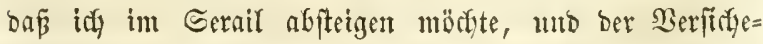

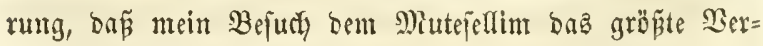
gnitgen madyen mitroe, weil fitid berjęlfe baburdy in ben Ctand gefeget fäfee, mir mündlidy für ben Dienft zu banfen, bent iaf ify geleiftet bätte.

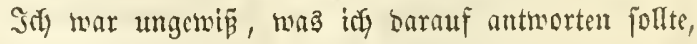

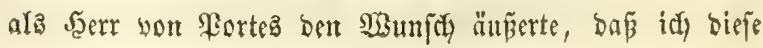




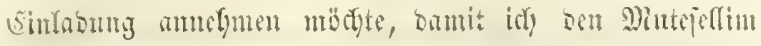

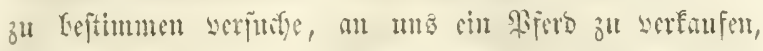

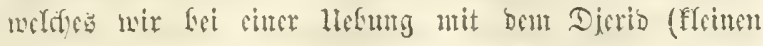

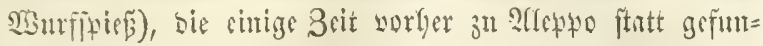

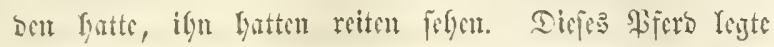
ficr Siroben bor gröpten Etärfe ab, unb war gant bejous

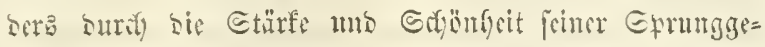
renfe merfmursig. Dafjecte geförte Ḧbersics ciner frei ben Sutron fefr berilfmten Fiprberace an, bie fei bon= jirfen unter bem Namen 2 (6) getenfe) frefinte ift.

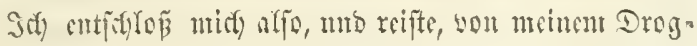
mam (Dolmeticter) Etewfens*) Eegleitet, ab. Sith ritt cin turfomanijuges Bĭerd, unb Etephems cincm Ejel.

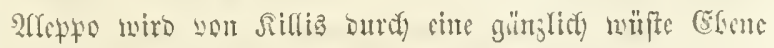
getrement, meldye ocmoch ziemlidy fruditsar ift, bemu is

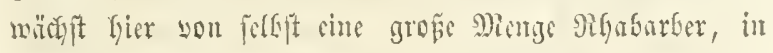

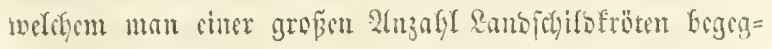

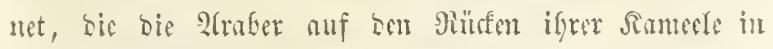
bic Etribte bringen, wo fie jeffige an bie Griecten wer=

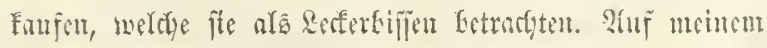

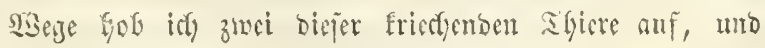

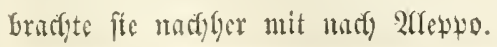

e) Stepficne war bor Solnn cines aften Franjofen, war 3น Af

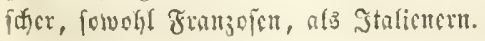


Bu Rillis angfonmten, ftiegu Etcubers แns id)

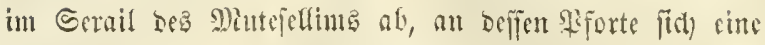
Shady befamb. Siann hatte mich bieje erbilift, als fie aufs ciliglte bie Jitdyt ergriff. In berfelbon erfannte id

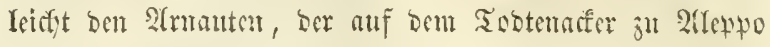

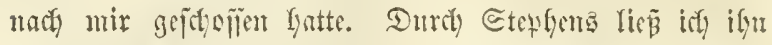

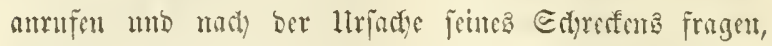

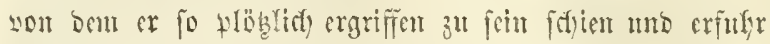
won biefent arment Seufel, saf ex firt) cingebiloet fütte,

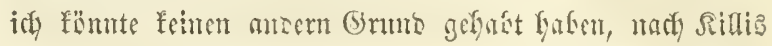

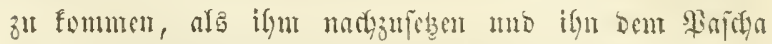

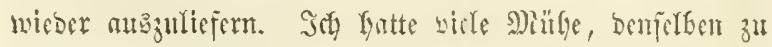

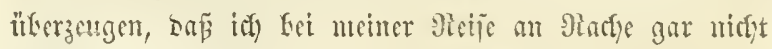

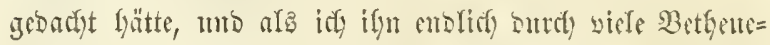

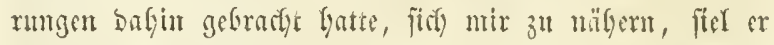

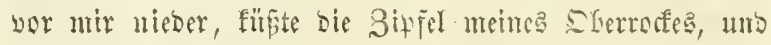

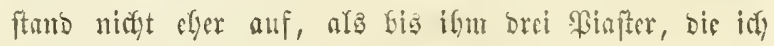
ifm in bie fand brürte, cublity bonicjen, baf feinte

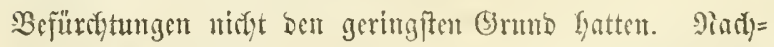
Den biefer fonderbare Sorfall sorüfer mar, wurbe id zu Dem Shatefellim gefülyrt. (5r befond fith in jeinem

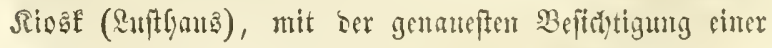

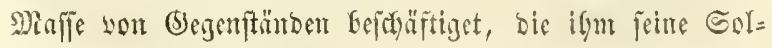
baten bergutrugen, un welitye bie Frudyt siner (siprefinng waren, nit ser or feime ltutertyanen zu belaften füe wïtyib sefumben batte. 2ra er uns zu Gefifut befom, fitand or

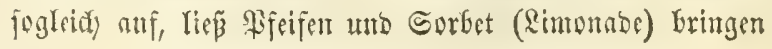




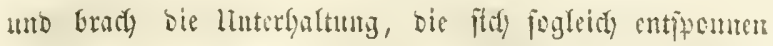

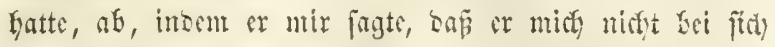

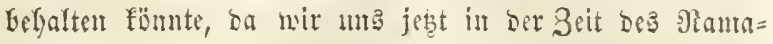

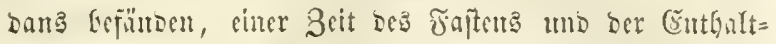

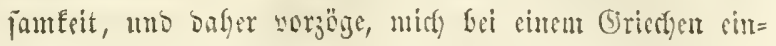

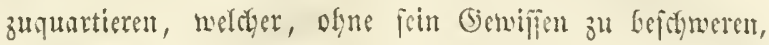

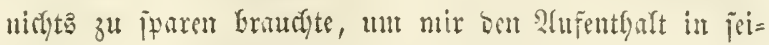

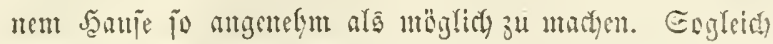

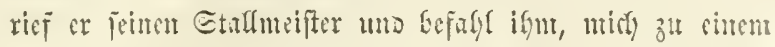
ber reiduften Griechen oer Gtabt zu fïfren, und emsigte

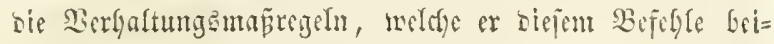
fïgte, mit ben menigen Sisortent: "Eage bent und Dem, ich ermartete, oaj er biejen Tranzojen eben fo gut aui=

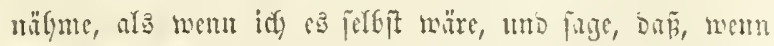

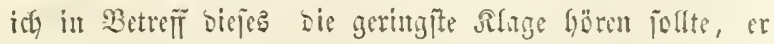

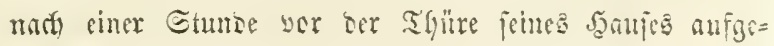
fnüџft jein müroe."

Der Etaflnteifter wollyog treuliti Diejen 2fuftrig, un

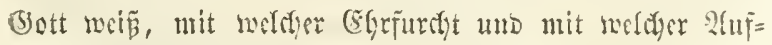

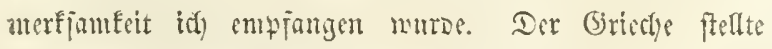

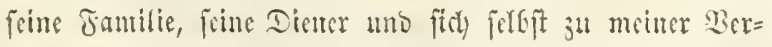
fügung, fowie er mix gegen 2lbent eirt gezeidineten (E)fermein auptringen Yief̧.

2tm folgenten Mlorgen Lengle idg mich bei guter Beit ill som Eerail. Die crite ornge des Slutcillims begog

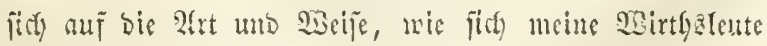

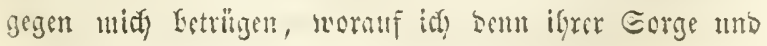




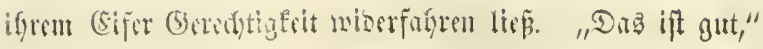

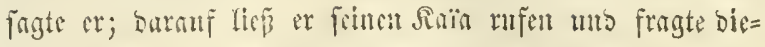

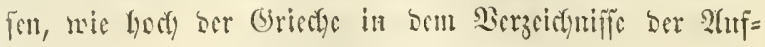
lage angefert tworden wïre. "Mit 2,000 \$infern," antwor=

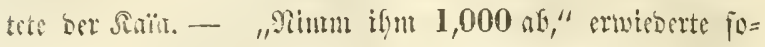

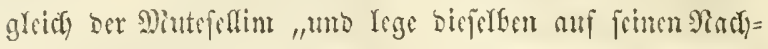
fur; sem idy barf nichte scrlieren."

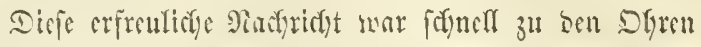

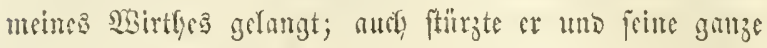

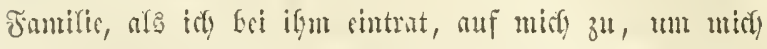

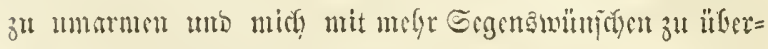
for üteren, are lich je erfortten latte.

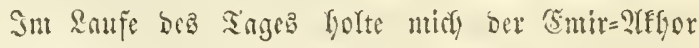
af, um mir bas \$ifers zu jeigen, son bem or in feintem

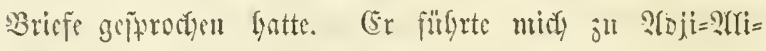

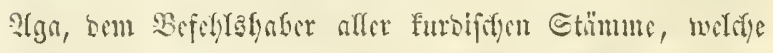

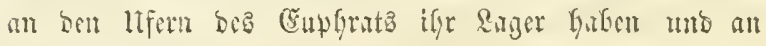
Sitlitis grenzer.

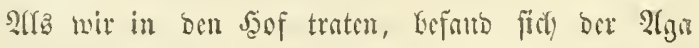

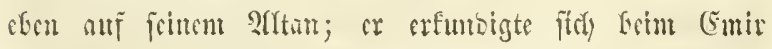

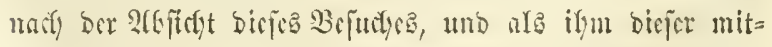

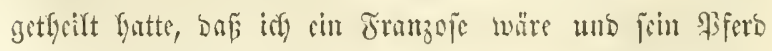

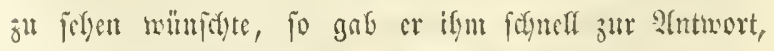

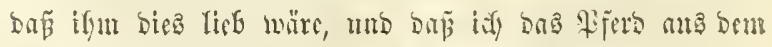

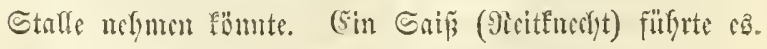

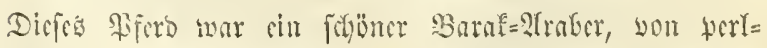

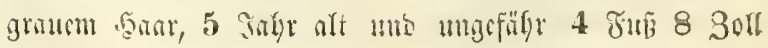




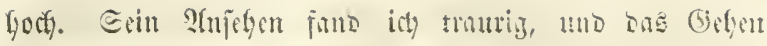

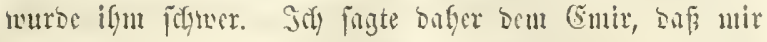

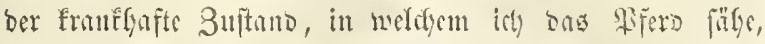

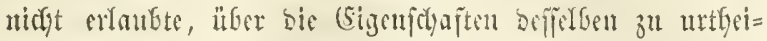

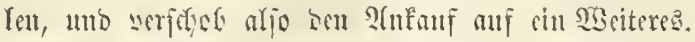

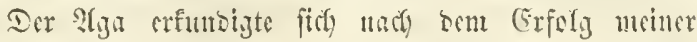

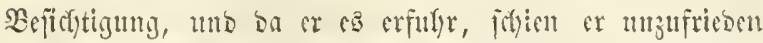

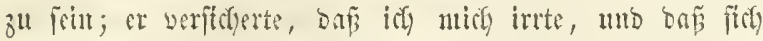

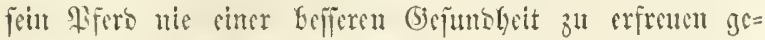
babt bätte. Darauf lief er mich bitten, zu ignt fyimanf

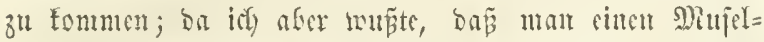

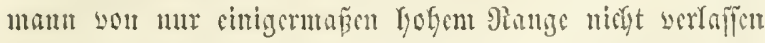

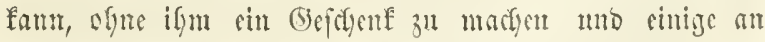

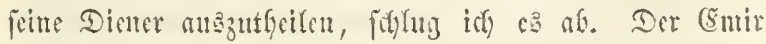

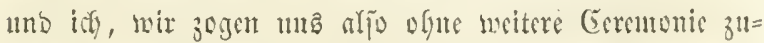

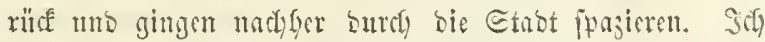

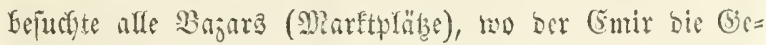

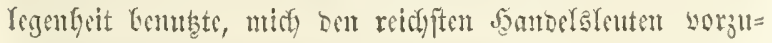

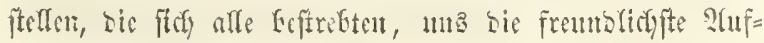
mafute amgedeifen zu laffen.

Diach biefen Bejudyen fïfrte midy oer (Smir ju cinem Eleimen Dorfe, bei weletyeu fitf bie Biferbe bes Diutefeflim auf ber Sacise befanben. Das Filo, wo fit meibeten, war mit nefrest aub Rantedbaaren genefiten Bilten um=

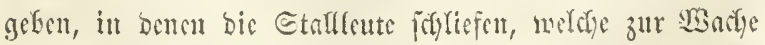
ïber biefe Thiere befeljligt maren. Sebez Sifero war mit Den wier Fingen, vernittelft boppelter Eqam 
fie Sufterf menten, befeftigt. Dieje Spannefinel füb you Strifen mit ciner 2trt von Filz umgeben, welden bie 2rafer mit bem Namen Ribfet Felegen; jebeß berielfen

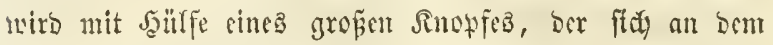

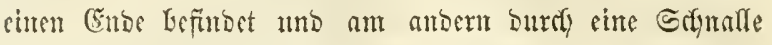
geft, bie ale snowflow bient, angelegt. Das eime bicjer હpannfeffel wird um bie Borber=, Sointerfïfe gelegt; bor Striff, melder diefelfon verbinset, ift genögnlidy jebr furz; and fund bem Thiere bie Jüne inmer fefre mafe feinem Ectmerqunfte gefradyt. Heber Dicfe erften Etramfoifel uno un jebes Eifientein legt atan vier anbere Spannefiel, wetre fid in einen langen Stric endiget, bet vorn unb fyinten freujweis geft und Dann an bie Dife cince in bie Crobe gefteffen sifernen

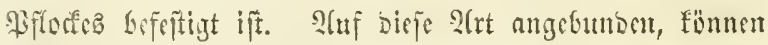

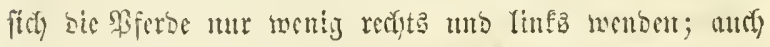

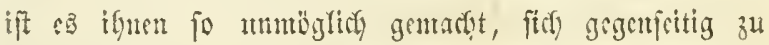
fidslagen.

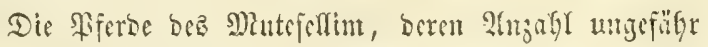
breip̈ig war, maren alle Barate 2 fraber, ober Surben. Eie

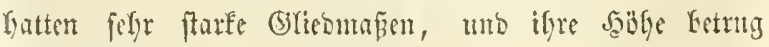

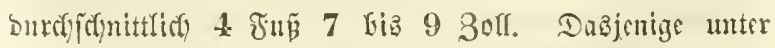

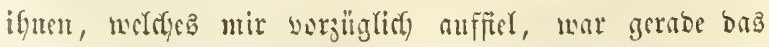

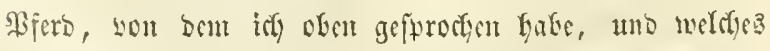

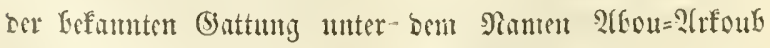

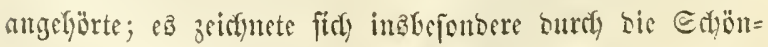
lyit frimer Sprunggelenfe aus. Sm Raufe ber lluter= 


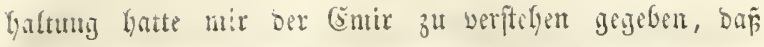
cer Nhutefellin beabfictigte, mir mit cinem ser Sferoc,

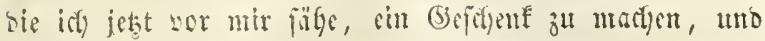

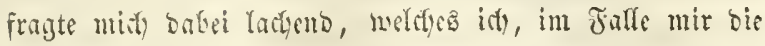

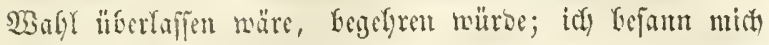

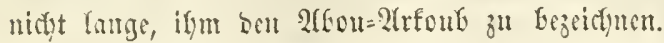

"Da gajt Du Medft," momottete or mir; "afer Du barfit baran nitft benfen, benn mein Setr ift bemperben ju jelle zugetban."

Eithon hatte idy bie bienitfertige (Ginfilifterung, meldye idy eben erwälnt bate, vergeffen, ars ben Morgen baranf, ganz früh, ber (5mir in meinent Bimner erichien und mir Ecridytete, Dañ ifym ber Mutejellim efen bie Albficft ange= fünbigt gätte, mir mit einem Fiferbe cin Gejedent ju

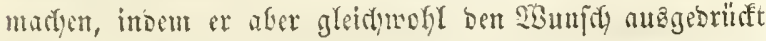
V)ätre, zur Ermicocrung sinen (5jegenitand zu ertyalten, Da= mit aurb ex cin 2lubenfen you nit fojäpe. Sith fugte

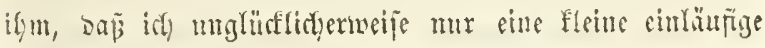
Sagbfinte mit cimem fleimen Bajonett bejübe, bie id aber

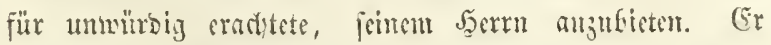
erjucte mith, ifin biejelbe zu zeigen, umb als or fie ge=

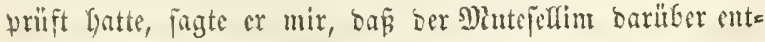
jüft fein twäroe, weil fie feidut wäre, und ex fith beblyalb

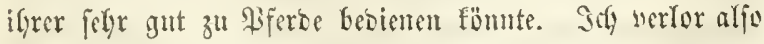
feine Beit, biefelbe ifur burdy meinen Drogmann (Dol=

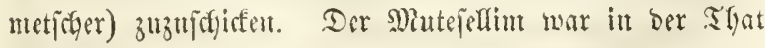

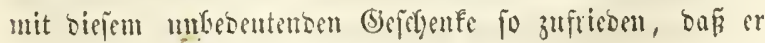




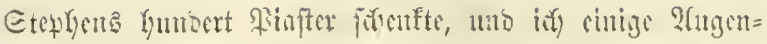

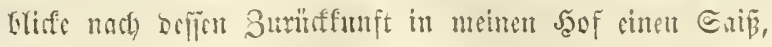

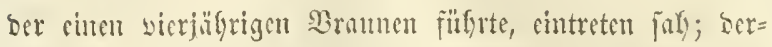

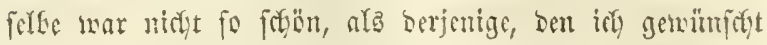
fantte, er war aber bemedy mit guten Eigenfidgaften regatet.

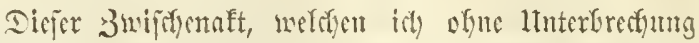

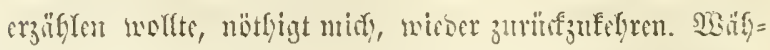

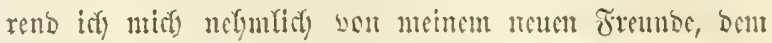

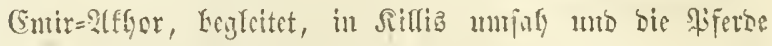

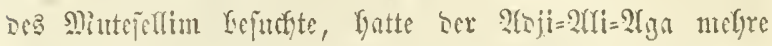

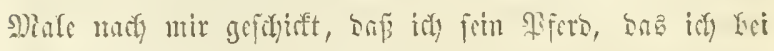

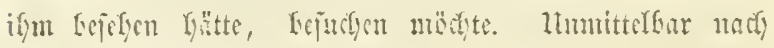

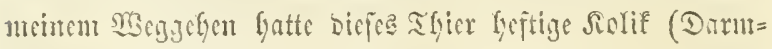

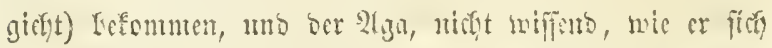

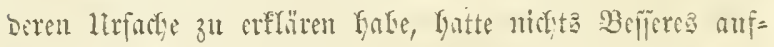

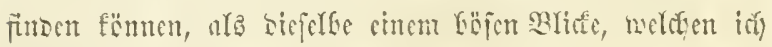

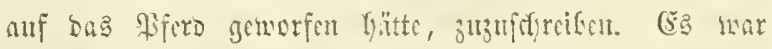

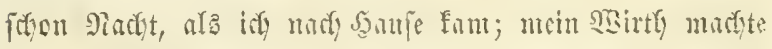

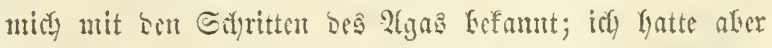

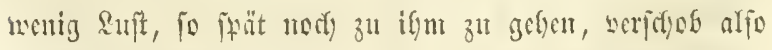

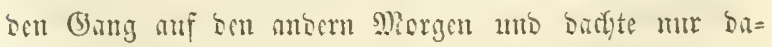

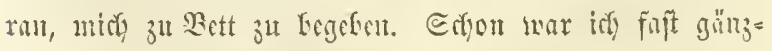

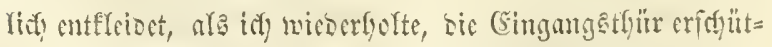

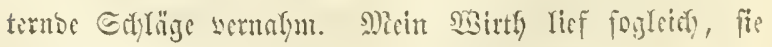

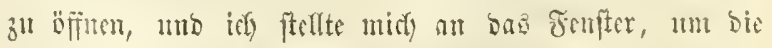

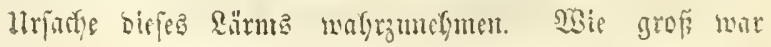




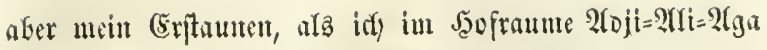
erblisfte, bent melyre Sflayen mit brenmenben Farfelt yor=

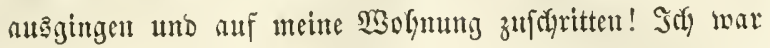

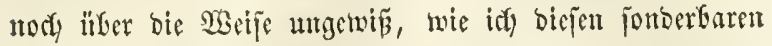

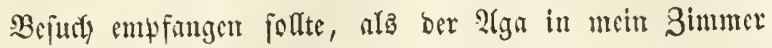
trat und midy fo bringend bat, fein Bjerb zu bejuctien,

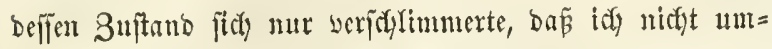
fin fonnte, ifm eine zujagende 2(ntwort zu geben. Sif) fleibete midy alpo auf bas forleunigite an, bemaffuete mith mit meinem Damasิencr, unb yon Eteplycus be=

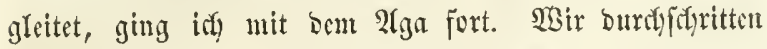
bie @tabt, inbem wix ung an ber Sanb Gatten, unb ars mix bei ifm angefommen waren, murbe id ofne Vergug in ben @tall bes Siranfen gefülyrt.

Da bie Türfen und 2trafer ber Ufer bez (Suphratz

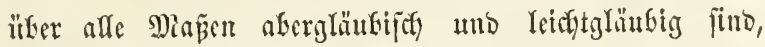
jo Gaben fie aud ftarfen (Slauben an bie 3auberei mo

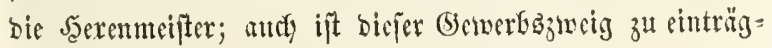
(ivi), un nitgt ausgebeutet zu werben; benn fo wie ein

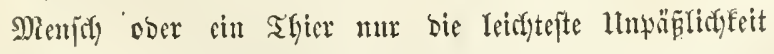
cmpfindet, wird fogleity ber Bauberer bea Drtes gertfen,

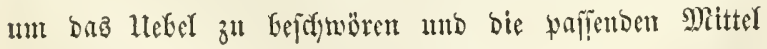
ou verorbnen. Der $\mathfrak{H}$ ga batte nidft ermangelt, bayon (b)ebraudy zu madyen; ber ber Bauberei $\mathfrak{B e f l i f f e n e , ~ w e l d y e r ~}$

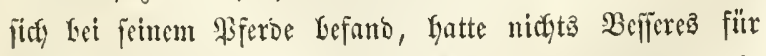
ratffant gefunocn, um die Siranfyeit zu veridueurfen, alz ben ganzen Rörper bes ßferbez mit Butter zu falben. 


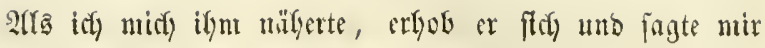
nit ciner triumphirenden Miene, Daś fen viel befier ginge. Dennody mollte idy ben Resteren

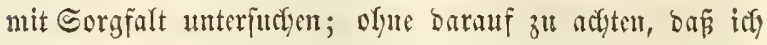
mir bie Sämbe befanterte, befüblte ich alle Theile und beobadyte mit 24 jumerfamfeit alle bic $\Im_{2}$ mptome, welche

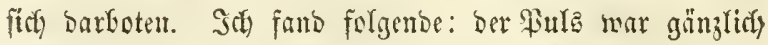

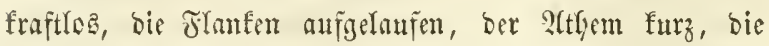

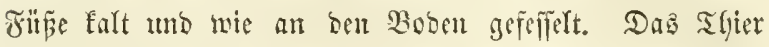

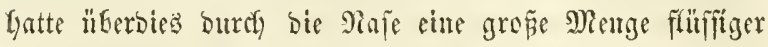

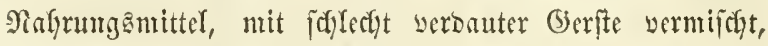
aแลgemorfen, unb cinen 2ugenbliff nati meiner 2(nfunft

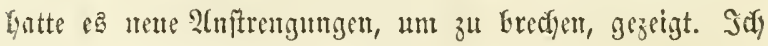
erfanute aljo feldr fiduell, bafi ber Magen geboriten war,

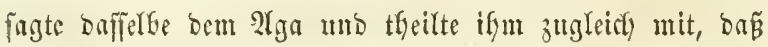

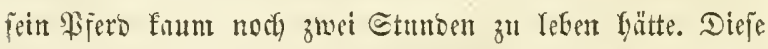
Sadyridyt, won meinem Dolmetiodycr mitgetfeilt, machte, Daß ber 3auberer eim Gelles Belädter aufidulug; bradyte aber beim 2/ga ein Siefïhl won Erftaumcu uno Sdyrefen berwor, worauf er micy bat, Dem Rranfen einige 2lrzuei= mittel beizufringen. Bergebens ingte idy ifym, baj alie Mittel untüt wären; or bejtand aber fo fturf und fo range Darauf, bis ich mich entịlopi, ifm ju millfafyren und cinen 2 loerlan zu madyen, uno nadybem bies gefdyelyen

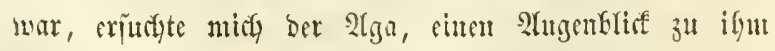
Gumañ z"l fommen; ich willigte ein und Etephens folgte mir. Co wie wir Drei in Den Empfangafanal getreten 


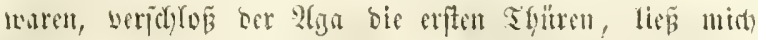

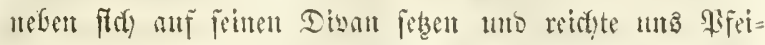

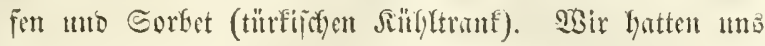

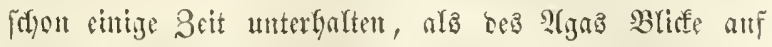
meinen Damasener fielen, mo er mir fagte, dá̧ berjerfe

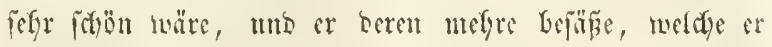

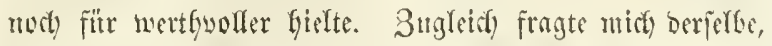

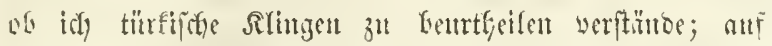

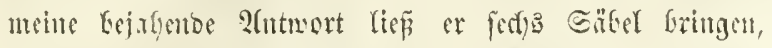

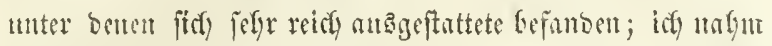

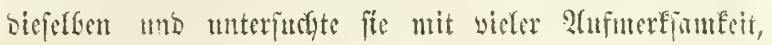

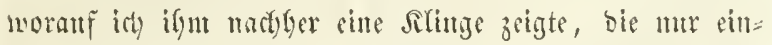

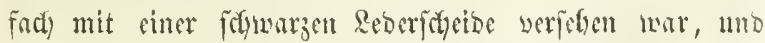

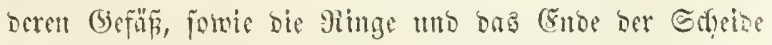
you bantascirtent (5ifen maren. Sch fagte ifm, ban bie=

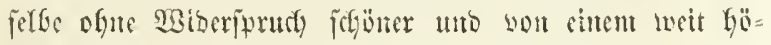

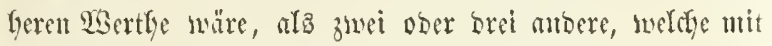
im Fencr verguldetem Eilber ausigelegt waren. (5r er=

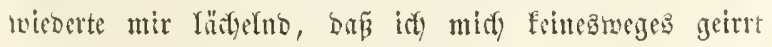
bütte, uno ergäblte mir, Dañ fie ifm als ciu srumb=

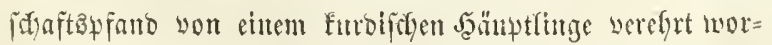

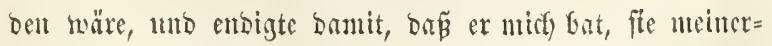

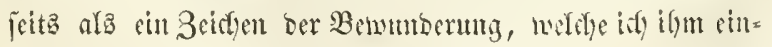

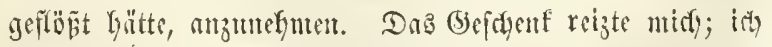
brannte vor Begieroe, ca in Empfang zit telynten; allein

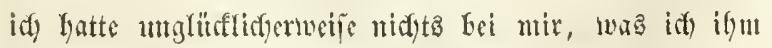

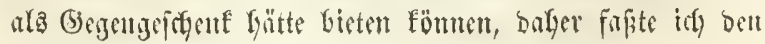




\section{8}

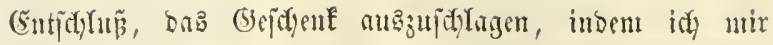

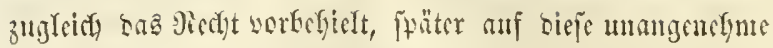

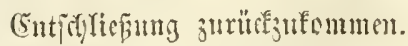

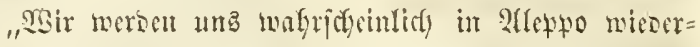
fintoen," fagte i(4), "baun wiro. uाz bie Beit vergönnt jein, แns öfterer zu fehen, um eine imnigere ซremmijouft

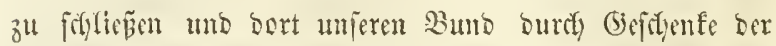
Jreunsfrfaft unb unjer würoig zu fefeftigen."

(Ga mat vicleiclyt noch nidjt cime uno eine balbe

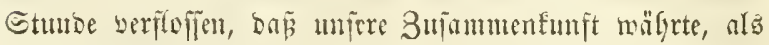

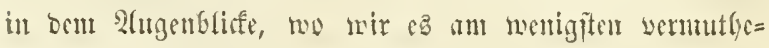
ten, ein Gflowe eintrat mo Den 2 liga benadyridytigte, sap̃

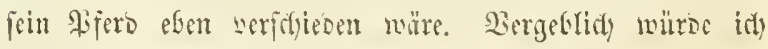

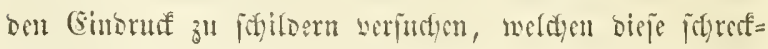

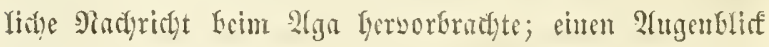

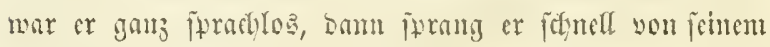

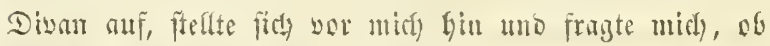
id) (5ott ober cul seurd mïre?

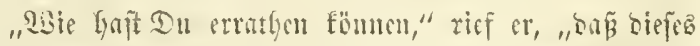
łfero jo jonell fterven würe? SBie vor Aflem yaft Du

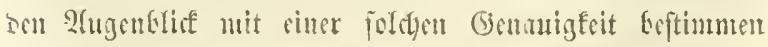
föแนtent?"

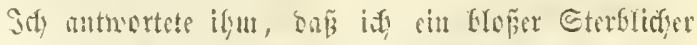

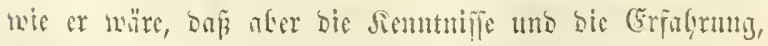

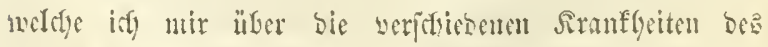
giferses z" cigen gemadit bätte, Ginränglich wären, un 


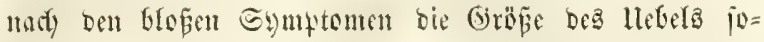

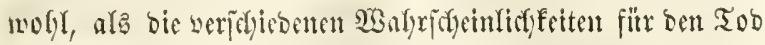
ober bie Gencinng, bie fid barnus ergäben, fuentbeilent

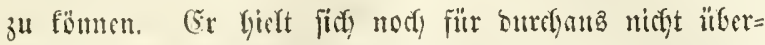
fïfrt und fragte mich, was bei feitent Bferde eit fo

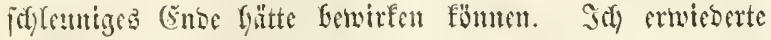
ifut, baj iff burety bie luterfutfung, bie idf yor bent

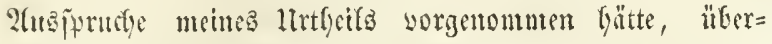

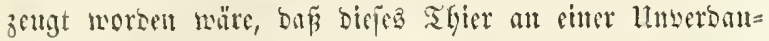
lidyfeit, weldye bie Berreifung bcs Miagene veranlapt

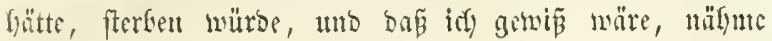
man bie Deffinung des Itnterfeifes yor, man in beffen

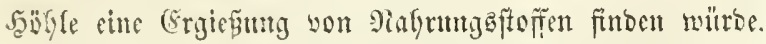

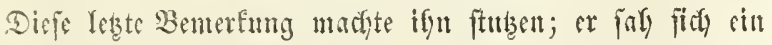
Mlittel igu barbicten, bie SYGarbeit meiner Befantumg fll erproben, tho fidtug mir vor, gleich ben Tag baratif

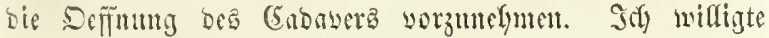
eill, unb ba er Gei ber Section anmejento fein wollte, ver= ntrebeten wir, che wir uns trennten, bie Stunte, zu mel= dyer ich ifyn abgolent mollte.

Die Sürfen vergreifen fich nie, wie befannt ift, an ciucm tobten Siörper, Denu bie geringite Berilbung ift

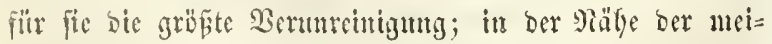
ften iffer gropen Gtibte lagent aber fajt immer cinige Sorben beibnifyer 2(raber, Deren :ame und Citten mit

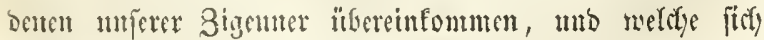

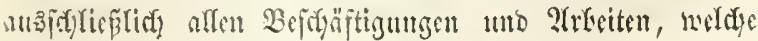




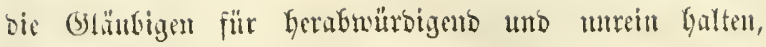
untergieflen. (Sime Sorbe folcher Bigetmer befindet fits

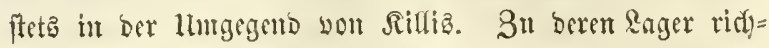
teten wir aljo, ber (Šmir=2lffyor des Mintepellim, ben idi mit ben Borfüfen ber vorigeil Madigt befannt gentad)t yatte, ber 2 gga mo idy umpere Sctyritte, unt ber erfiten

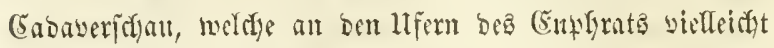

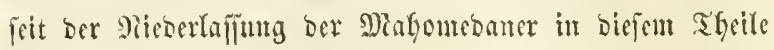

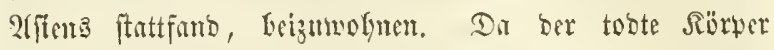
jợon längit vor uns angefonment war, hattent wir micht

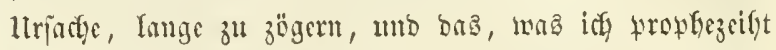
Gatte, traf iu allen Simften eill: Der Magen war in oer

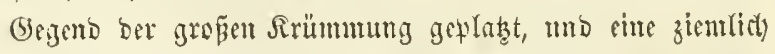

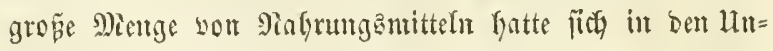

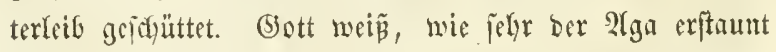

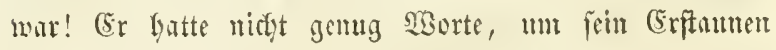

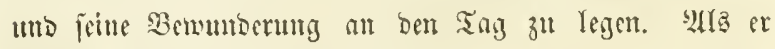
fich berufight batte, fodfug or mir wor, mit ifm nadd cinem

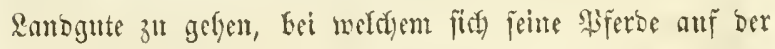

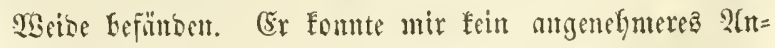

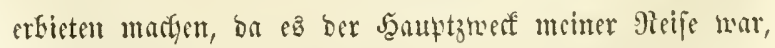

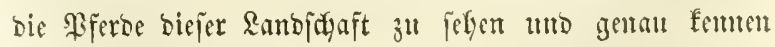

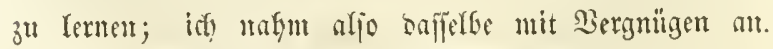

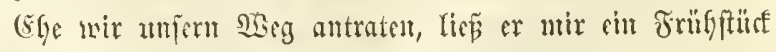

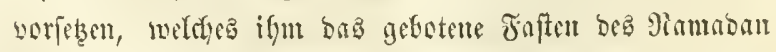

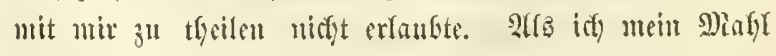

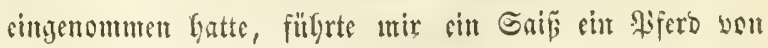




\section{1}

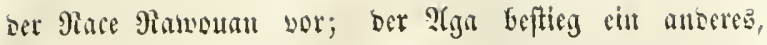

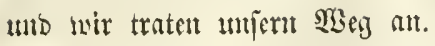

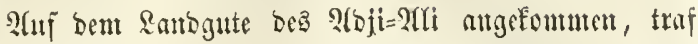

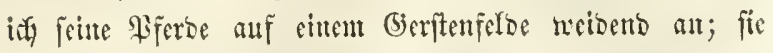

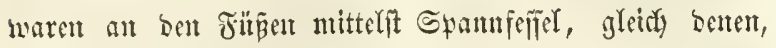

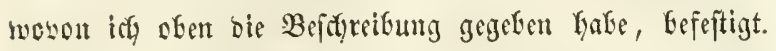
Der 2 (ga bat utich), fie ju unterfuct)ent nthd zu ferlyen, of

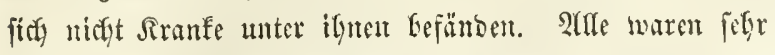
fett, unto megre unter ifuen, weldye ferge rotge Afugen uno

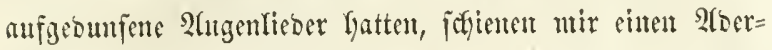
la

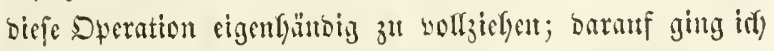

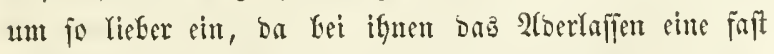
unbefantente Sache ift. Sie gefrauchen bazu als sinftut=

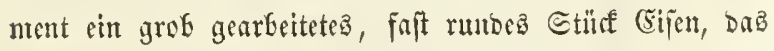

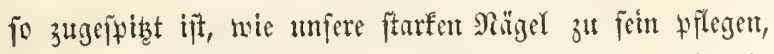
und burdy beffen Seft zientrich einem Erfmepper ägutelt.

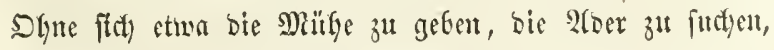

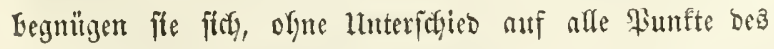

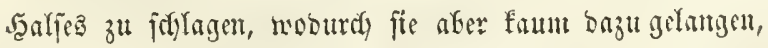
sinige Trenfert Blut z̆ll laffert. Dafer formte Der Hga

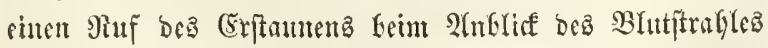
nidft unterbrïrfen, weldfer auf cimmal ats ber A(ber, bie

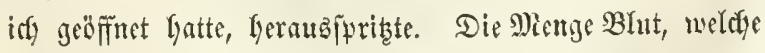

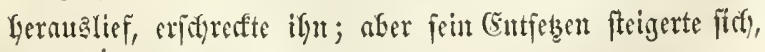

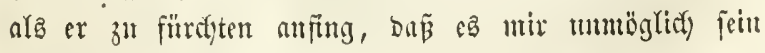

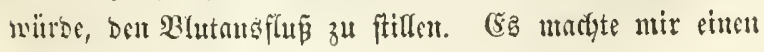




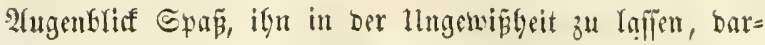

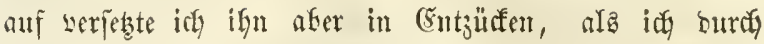

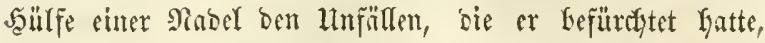
wlöblid cin (Enbe ntadyte.

Eines biefer Xiferbe latte cine Eprunggelenfausa

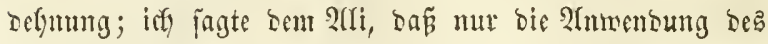
Feuers biefem Hebel ablyclfen fönte; worauf cr won

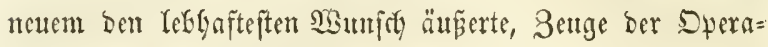

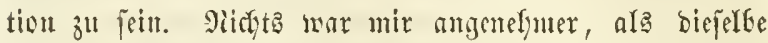

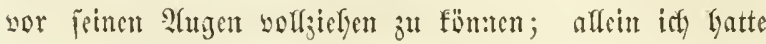

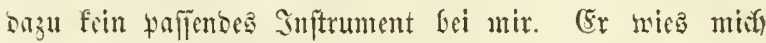

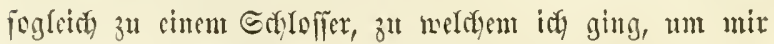

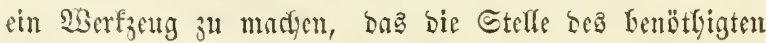
wertreten fömnte, unb als mir bicz gelungen war, verrict)= tete ich gleich barauf bie Dperation.

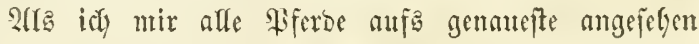

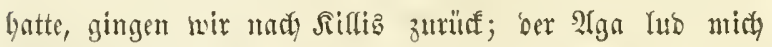
zur Mittagstafel ein; allein ber গamaban crlaufte ung nidyt efor, als mach Gonnemuntergang zur Tafel zu geben. Diefes türfificy worgefetste Magh beftand auts ben ausge=

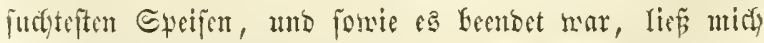

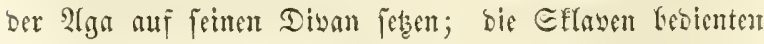

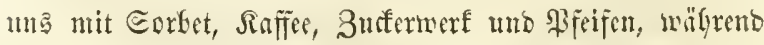
fitif die unterbaltung ïfer die Biferoe entipann, weldye

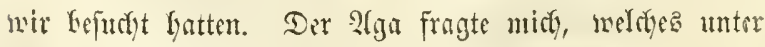

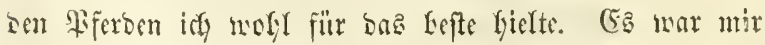

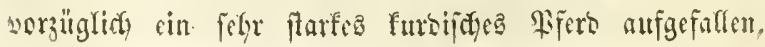




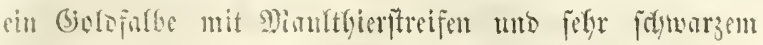

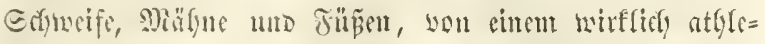

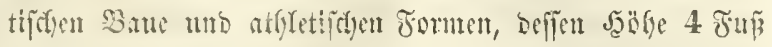
9 Bofl betragen fomute. Sidy ftanto arjo nirdyt an, ilym biefes Sifers are basjentige zu bezeirfunen, welctent irg bent

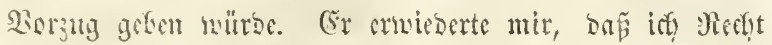

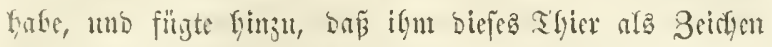
oer Fremtujofyft vou bent Edyeif eines furbifiden Stant=

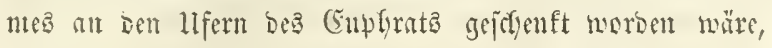

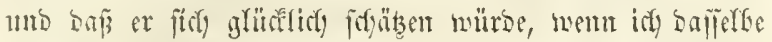

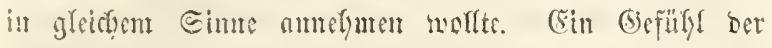
Beffyeisenteit, Das idf feitom oft bermet babe, berwog

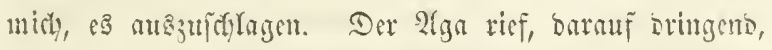

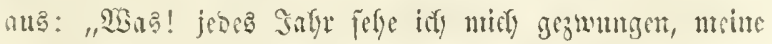

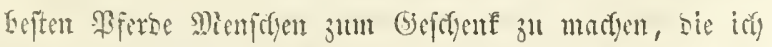

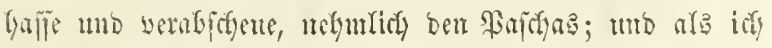
zum erften Male in meinem Refen einem Manne begegne,

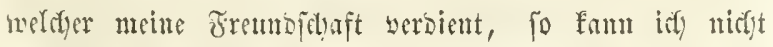

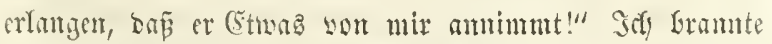
vor Berlanger, das Prero anzunefmen, mar alp̣o fejt entifyloffen, mirh ïberreben zu Inffen, wib ertwartete nur

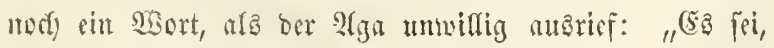
forectien mir bayon nicht meiter!"

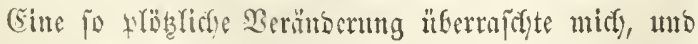

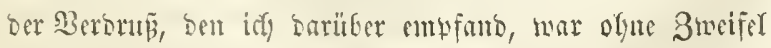

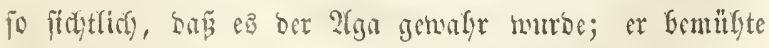

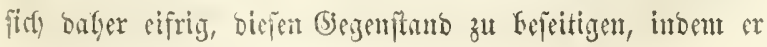


Giusulfingte: "Sith will Dir einen Dientit erjeigen, wetrfen Du wenigftens nidyt aแbjofilngen fanmft. Dab Ranto, wel=

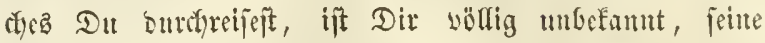
Epradhe wie frite Eitten find Dir ebenfalla frento; Du

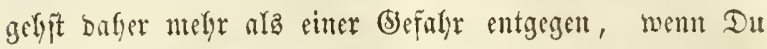
midi) werfaffen mirft, un Siferbe bei ben weristiebenten

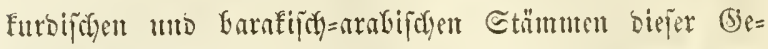
genten zu juffent; foft alfe finto mir entweber untergefon,

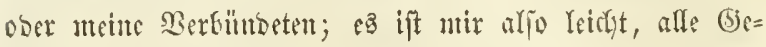
farben, weldye Didf babei bebroben fömten, won Drincr Sieife ju entfernen. Un biepę ju bewerfftelligen, treroe iif) Dir cinen nteitur Surben mitgeten uto ify soricfe

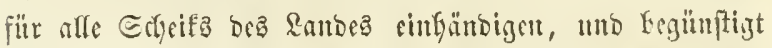

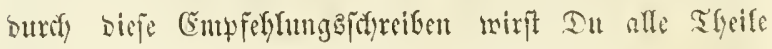

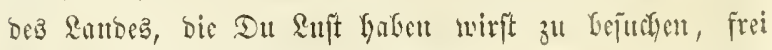

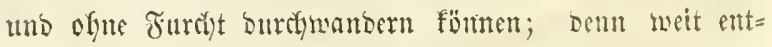

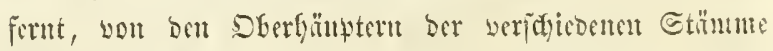

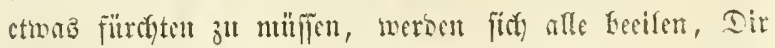

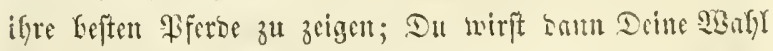

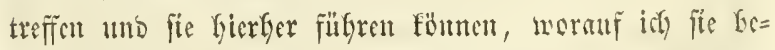

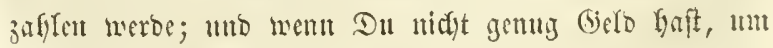

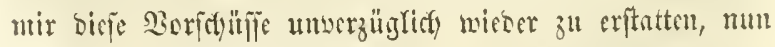
fo wirft Du mir biejcleen und) Deiner 2tufunfe in Frant=

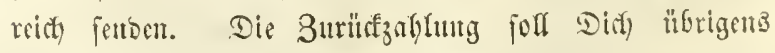

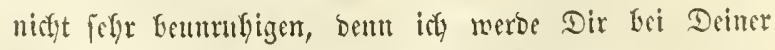

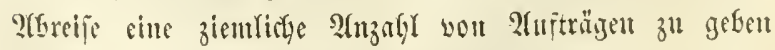
und Dich zu bitten Gaben, nuir mefre Begenftünte ata 


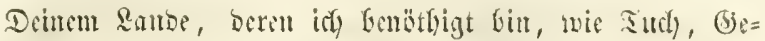
wellye utto bergleidyent mefje zu jenten."

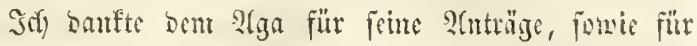

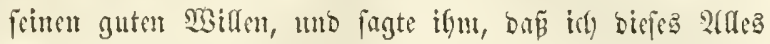
fefyr gern angenommen bafer mürbe, nem mid) nivat

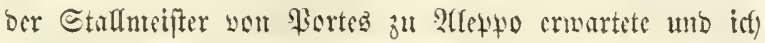

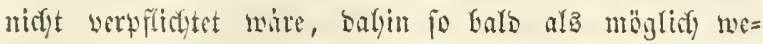

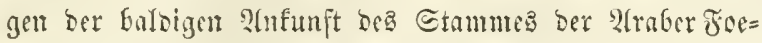

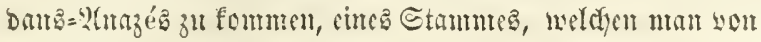
cument Tage zum ansern foine Solte in bem Thaile bor

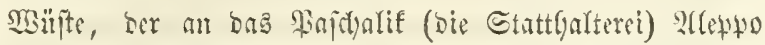

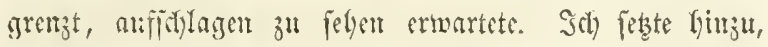

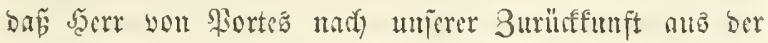

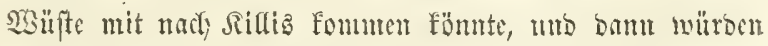
wir you ifm bie Berwirflicfunty feituer Berporechungen in 2 (niprut) nefymen.

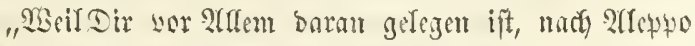

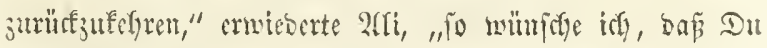

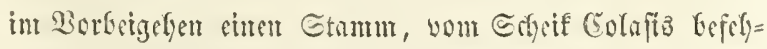

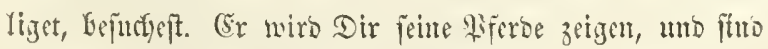
unter benficteen joldese, bie Didf anjpredfen, fo wirb er fie

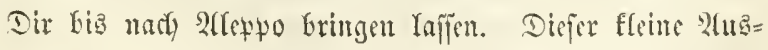

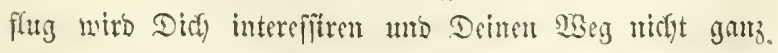
um cinen Tag verlängern; bafer zeige ntir unt bert Tag an, wo Du afzurcijen gedenfif, bamit idf cinen Surben zu Deinem Befelyt ftellen Fann."

S(t) jagte ifm, bo 


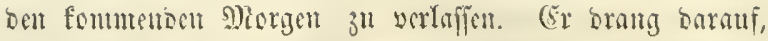
Dafi idh nod) cinige Singe jugeben möchte; allein ars or

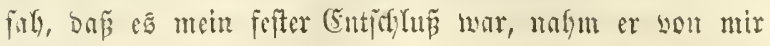
?QGituied.

Eefyr zeitig war ich anşgegangen unb ben ganzen Tag nicht in meinem Suartier erficticnen; als ich zurürf=

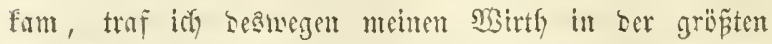

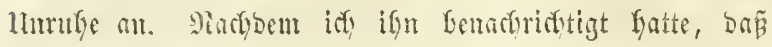

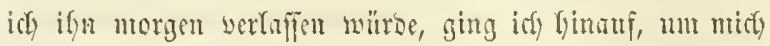

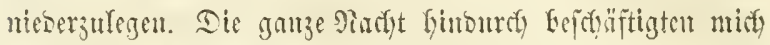
afer mu bie Ereignifje ofa vergangenen Tages, und ids)

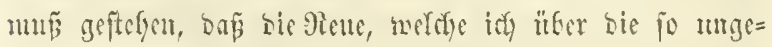

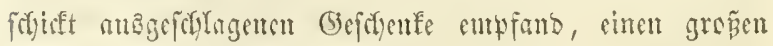
Theil menter Betradytungen atsmatyte. Scty fdylief we=

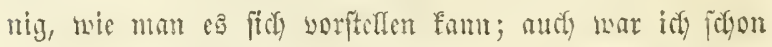

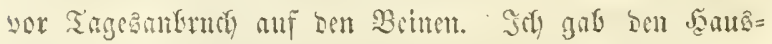

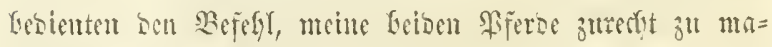
dyen, z̈̈nbete mrine Şfrife an umb wollte, um bie suft

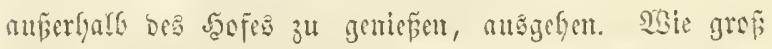

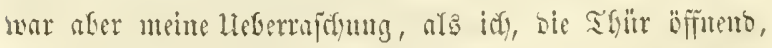

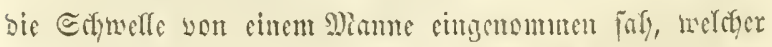
ansgeftresft altf ber (Srbe rag, und in Ser cimen Samb cun Gerfretfen, in ber anbern bie Reiute eutes neben ifm

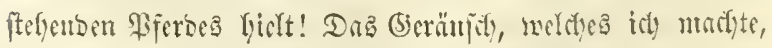

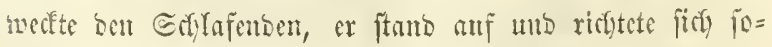

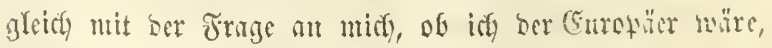

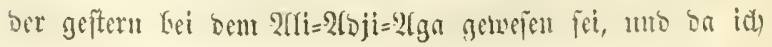




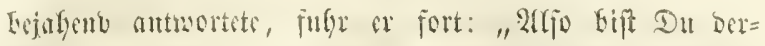

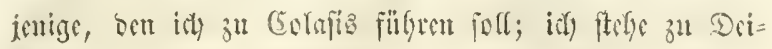
ment Befefyl."

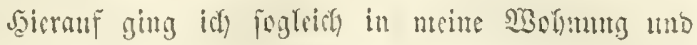

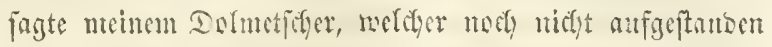

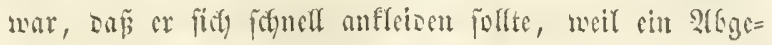

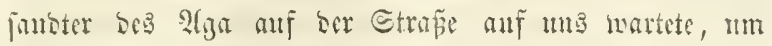

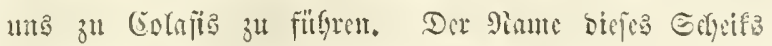
wirfte afor wie cin eleftrijher Eeflag anf ifu.

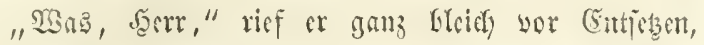
"Gie matfer zu Eolafia geffen? Gott beföte Sie Dayor!

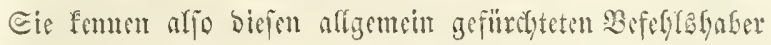
nicyt? Das ift rim Barbar, bor Gie, ebe Sie Beit baben werben, feit Bett zu betreten, unfringen mirs! SBoffen

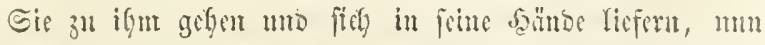

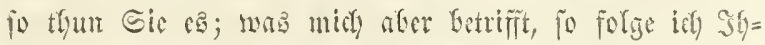

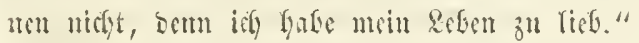

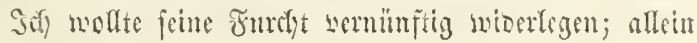

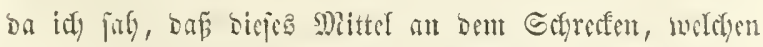

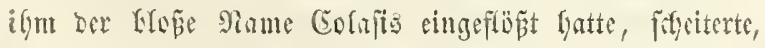
ungut ich zur Drofung meine. Bufluctet und fagte ifon, oafi, wem er nidjt int Guten folgen mollte, fo müroe

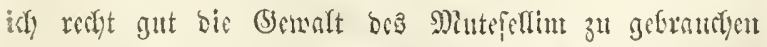

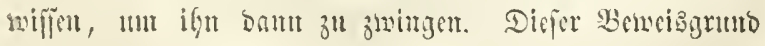
ge्clung mix.

"S⿱

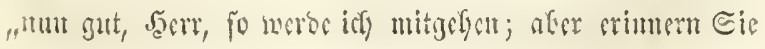




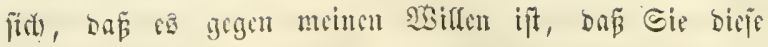
verwünfte Sicip antreten; Gie merben mcincm Sotf und

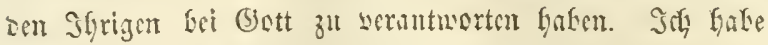

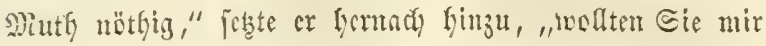
mobl zu befien 2tajeucrung cin Glas Bramntmein geben?"

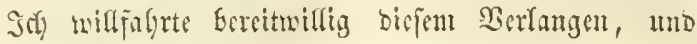

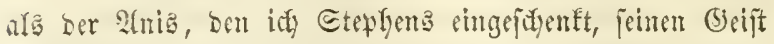

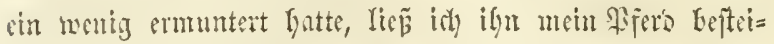

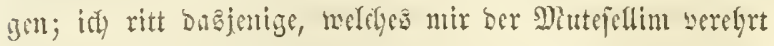

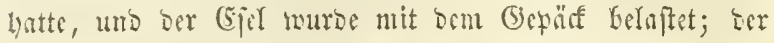

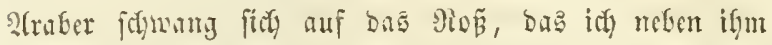
gefefen Vatte; neine Sirtgalcute füpten mir bie Sănoc, gabon mir ifren Gegen, und umjere Eleme Saramane jebzic fict) in 9liaridy.

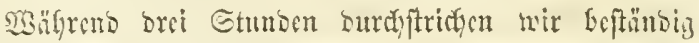
cinen trofenen und gü̈jlid whiften Bosen; wir Eanten Darauf an cincn Eleinen Barey, an Deffen lljer wir einize

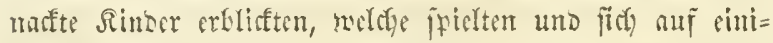

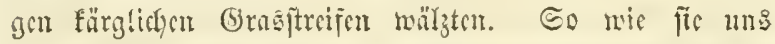
gemaly murben, nafmen bief Sinberden bie Flludgt umb

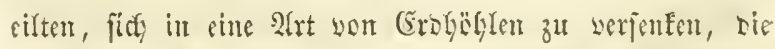

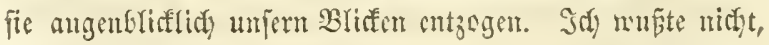

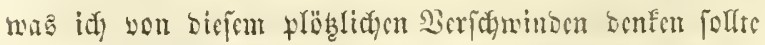

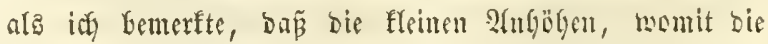
Ebene greichjam befäet mar, bic Därber ebenjo vieler

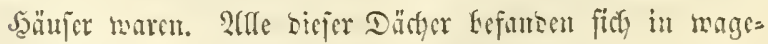

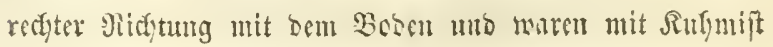




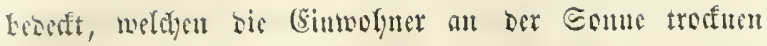

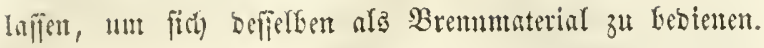
Indent wir unjere Bliffe etwas weiter ridjteten, fah idy

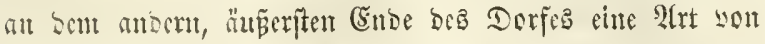

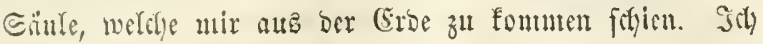
näberte midy serjelben umb erfunte in siejer ponberbaren

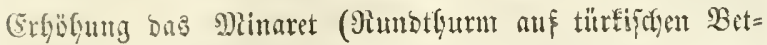

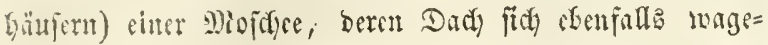
rectit mit bent iterigen Groreidye befant.

lluter Surbe fragte midy, of idf effen mollte; auf utcinc bejatyende 2(ntwort fprentigte er gerabe nady ber

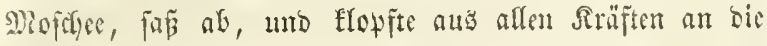
sGüre, morauf fie ein alter Edyeif b̈fnete, inbem er fragte, mas er mollte.

"Sct) mill, Dáp Du uns Crier, Brot unto Datteln giebft," jagte ifym ber Sirbe.

Der Satyeif jonien einige Sefmierigfeiten madyen z" nolfen; aber bor Rurbe berrolyte ifn fogleich mit feinem

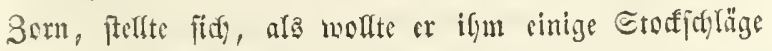
geben, morau ber (Breis, zaglaft gemadyt, lyineinging und

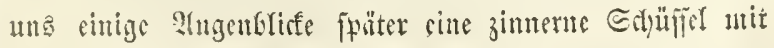
einem Dutbend in Butter gefratenen Eien belegt, ein wenig joledjtes Brot ofue Gaucteig, Dattelu mo eiutige

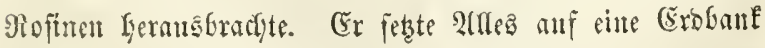

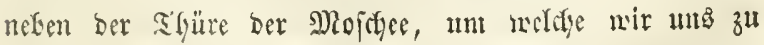

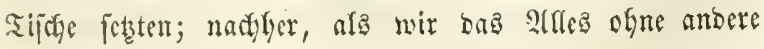
Şülfe, alz bie umferet Şänbe lyatten verjdywuben Iafjen, 


\section{0}

reirfyte unt sin Gflave sine 23aje won 3inf, weldye mit sisaffer autgefüllt war, Defīen woir una zแแ Trinfm mo

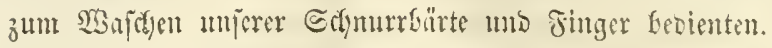

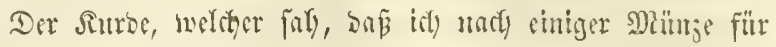

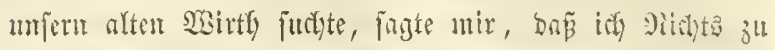

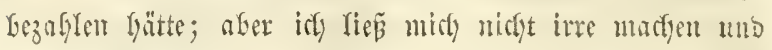

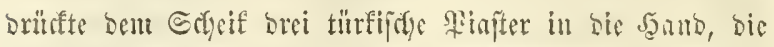
Der Greis mit Den Beiryen ber rebgaftejten Ianfentfeit aแmalym.

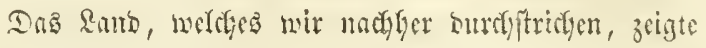

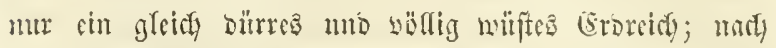
eitem Maridye sou brei ober vier ๔tumben famen wir cmb=

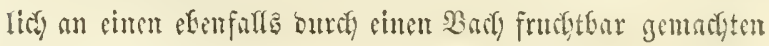

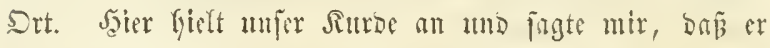

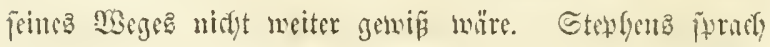

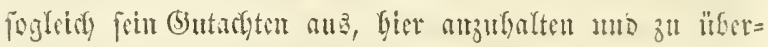

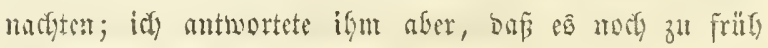

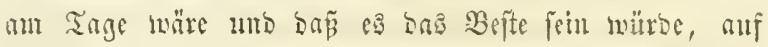

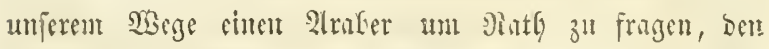
idy in ber Jerne cine Secrbe B̈̈ffer bewadyen fary. Cte= whens ftand an; body idy fetse fogleidy beide Sporen sin urb galopyirte mein Siero macy oer gitbtung gin, wo

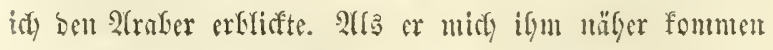

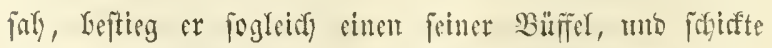

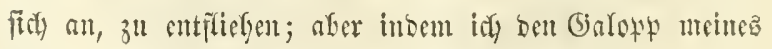
Sfrenbes nod) mefir bejdyleunigte, gelanis es mix, ilgn ein=

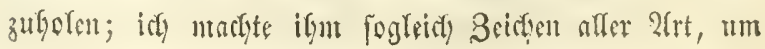




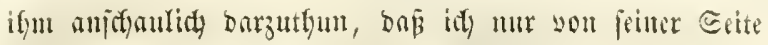

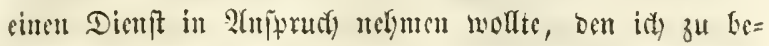
loknen wifjen wïrbe; allein all mein Geberbenpiplel feyien an feinem foflectyten SBilfen, ober an bem Mangel bes Berftanbez zu jofeitern, als mir einfiel, ifm cinen jhani=

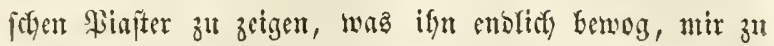
folgent. SWir ritten, indem wir Beibe bns ticfíte Still= jodweigen beobadyteten, bis wir bei Stephents, weleder mir won weitem gefolgt war, anfanten; biefer Retztere fez̧te

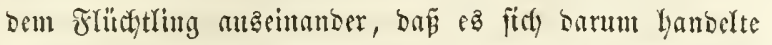

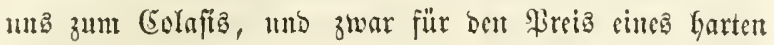

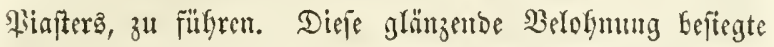
alle Bebenflidffeiten bes Arabera, welefer fitd) nugenblift=

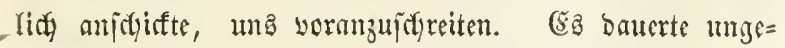

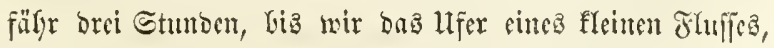
unter bem Samen Somaio befannt, erreidyten, weldyer

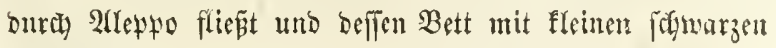

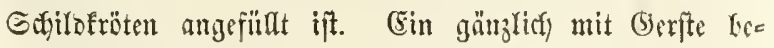
bauter bägel erfyob fith yor uns an jenfeitigen llfer. Bei unjerer Ânïferung fungen brei Stuten, tweldye wir

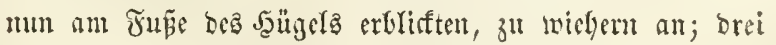
Surben, bie ofyne Bmeifel in Der Mitte Der Sierfte lagen, ftamben fogleich auf, fprangen auf bic Stuten, fulyen unz

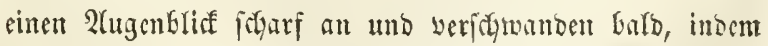

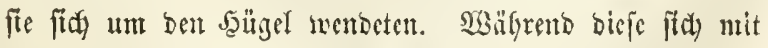
ber gamjen Єafuelligfeit ifyrer Яiofle entfernten, ging unjere fleine Saramane an ciner feidyten Stelle, Die uns der 


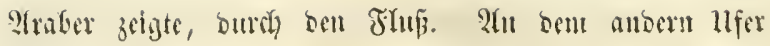

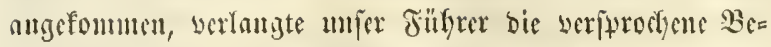

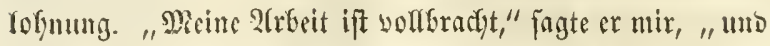

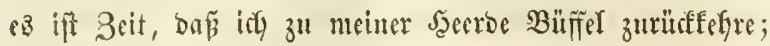

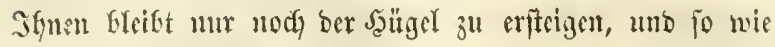
Sic befien Bipfort erreidyt Yaben, fo werben Sie unter firts

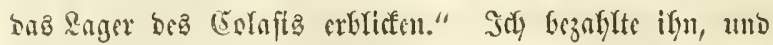
or ging zurürf.

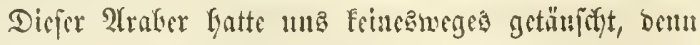

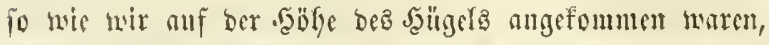

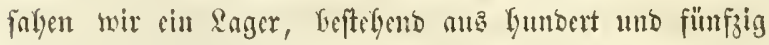
3elten yon graulidyer farte uld mit eincm Gemerfe yon simeslfigaren beberft, wor ung. 


\section{B) ittes Siapitel.}

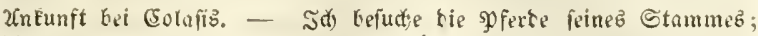

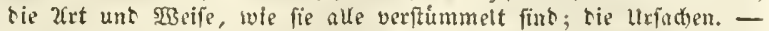
Rurtifde Frauen; id bin nahe taran, von einigen unter ignen beraubt zu werten. - Sameelbutter; Sie 2rot, folde zu bereitem.

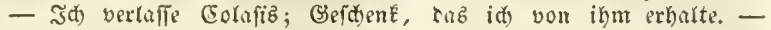

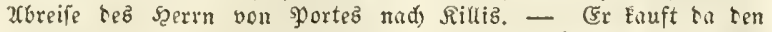

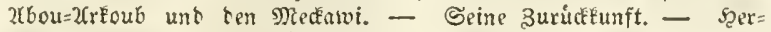
umirrente Scunte ter turkifoen Státe; ihre Sewolnhbeiten. Bergebrider Sergiftungžverfud an einem terfelben. - Sonterbar: TBirturg tez Suedefilber=Subtimntz. - Uebung mit rem Dierio

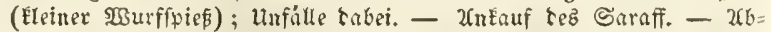

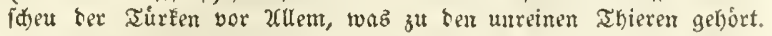

Die brei Rurben, weldye wir un ben fleinen Berg yatten werben fefeu, waren ofne Bmeifel yon Eo= lapte vorgefdobene Soften; fie mupten fely wenig Beit gebraudyt Gaben, unt bie med ziemlidy lange Etrecfe, bie fie onrdylaufen mü̈ten, jurïrfulegen, denn wir Gatten faum Brit gefabt, bie Bulte zul zăblen, aus bonen bas Eager teptand, als eine Bemegung unter ben Surbon, bie oaffelbe bewohnten, ftattfans, ruto beren wir cine beboutenoe Babl, mit Säbeln umb Ramjen Eewafrutet, aแf mis juffrreiten fafyen. Diefer Eefyritt, ber fofwer fu begreifen war, Gradjte Eutieben in Der eben nidft feldenmütgigen

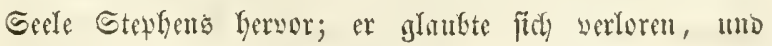
faum Gatte er bie Sraft, sent F̈̈lfer, weldyen mir 2roji2)li=2lga mitgegeben batte, in meinem Sianten zu befiglen,

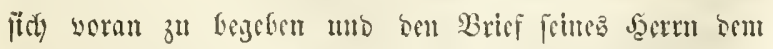

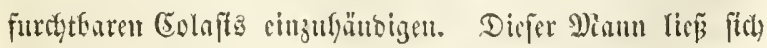

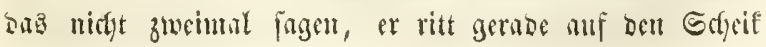




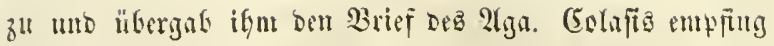

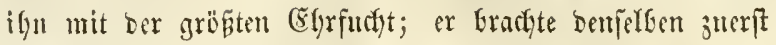

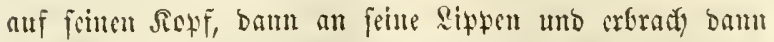
Deffen Siegel. Daranf lab er ity, inbem er feinen (b)ang fortictete, rumb als er bei mir angefommen war, Fejafy or

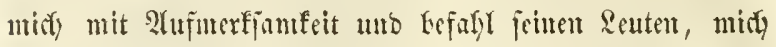

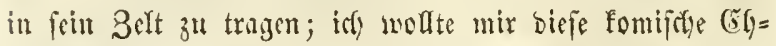
rentezeigumg serbitten, allein iti) murbe miber meinen ŞGillen von meinem Eattel beruntergehoben. (Ein Surbe

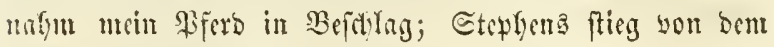

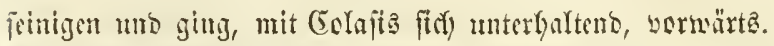

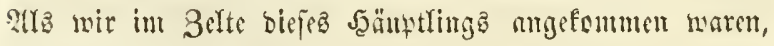
Ereitete man cimen froctituoflen Tetpict) anf ber (Erbe aus,

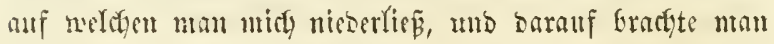
mir nod ein ziemlidy fäünes Sijpen won rotfen Ent=

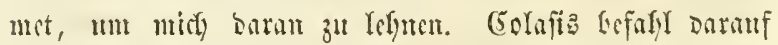

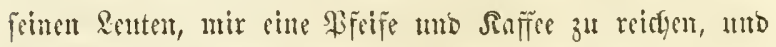

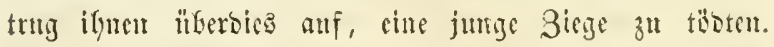

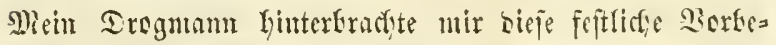

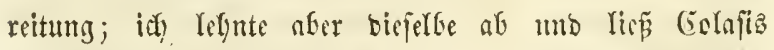

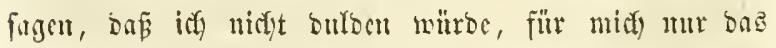

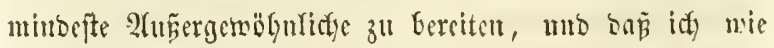

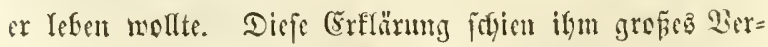

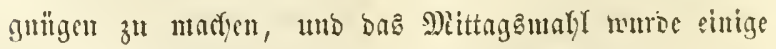

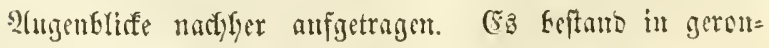

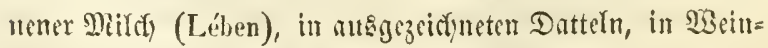

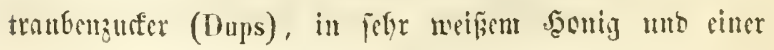




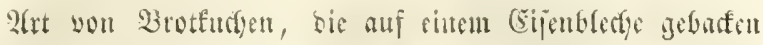

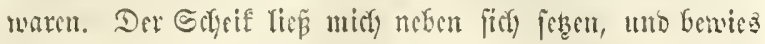

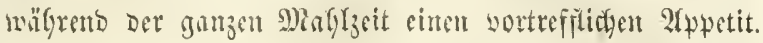

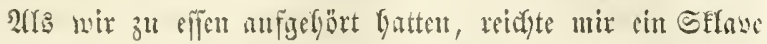

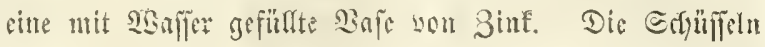
murben you ber Strofyuattr, widde uts als Tififtud) unb Tafel gebient fyatte, abgerïunt, und man foradyte uir

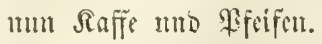

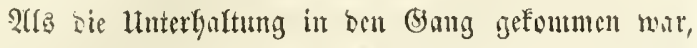

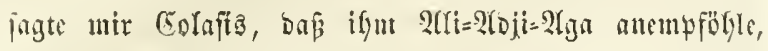
für mich bie größte Gorge zal tragen, fowie ifm bäte,

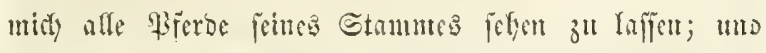

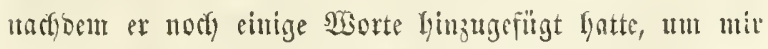

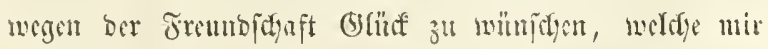

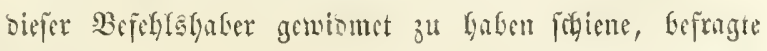
er midf, of idf unmittelfar bie Vienter jebes feituer Sur=

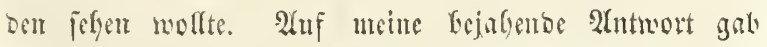
or Befegle, ntito balo jog jeber Surbe, jein Fifero am 3rume füfrenb, bei mir voribler. Eomie bieje Tgier:

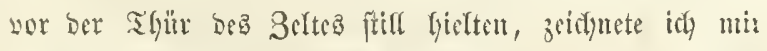

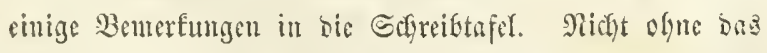

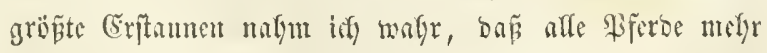
soer weniger berfithumelt waren; benn eintigen waren bis

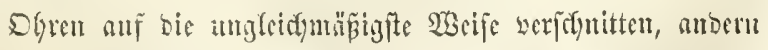

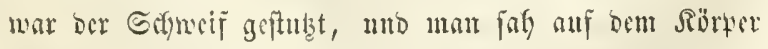

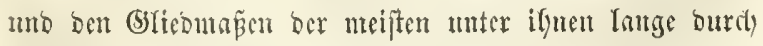

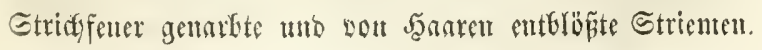




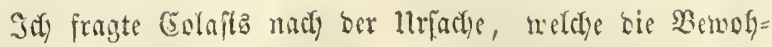
ater jeines Ctammes bazu veraulapte, ifre \$ferbe fo zu (đ)

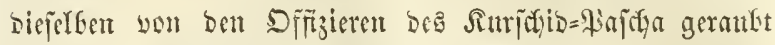
zu jegen, gefoülye, sie fitch ofne bicpe Berftümmelungen

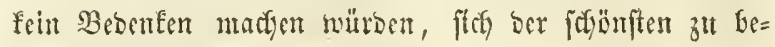
mächtigen.

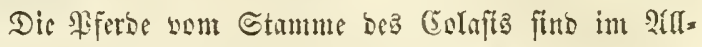
geneinen von nicft grofent Bane; fite haber faft alle mur

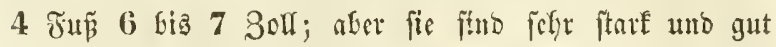
funbamentirt. Das Seren ift zwar etwas Sreit, Docf ift

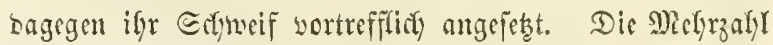
bat ganz braunes ober völlig sunfelfirfobraunes \$aar.

2rla ich alle bieje Pferbe genau feịegen Gatte, fragte midy ber Edyiff, weltgę biejenigen wären, bie idf yor=

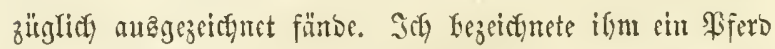

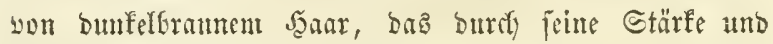
Feftigfeit, forie burfy Edyenteit feinter (s)lieber mir bejon=

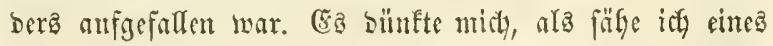

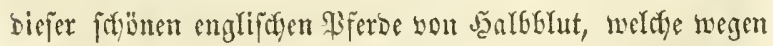
ifrer Rraft und Dauer fo gejubt meroen. Seine Figur war bei weitem gröper, als bie ber übrigen Pferbe (ę

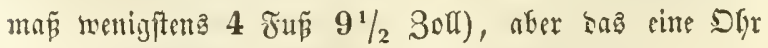

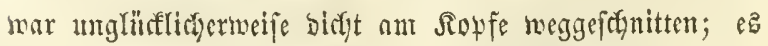
batte gebramte Striemen über ben ganjen Sörper, und feine Beine waren mit tiefen Marben beberft. Giegen

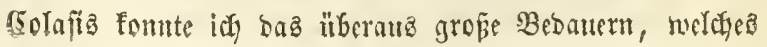




\section{7}

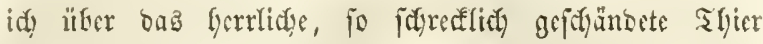
emtafanto, niçt verläugnen.

"Das ift in gerabe jein Borjug und fein Berbiemit,

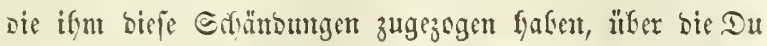
Didf) breflagit," jagte mir ber Styeif; "Dentr wäre es

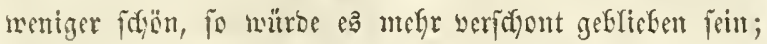

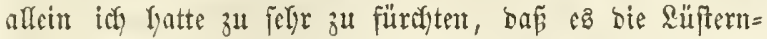

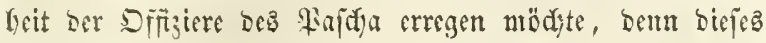

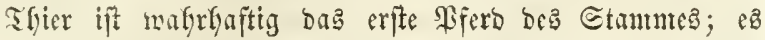
Lient als Bejuäler unt belegt bie beften Etuten. Miorgen werse idf Dir eninize feiner Nact) fomment jetigen, und idf

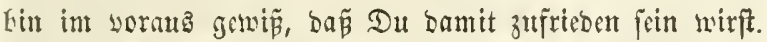

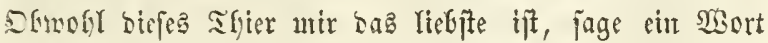
turb 距 ift Dein."

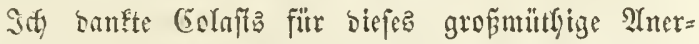

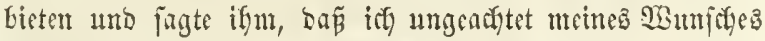

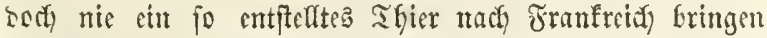

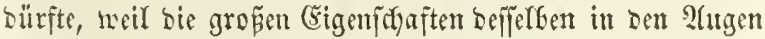
meiner Rands scute unter biefen entfefflentoen Marben, mit

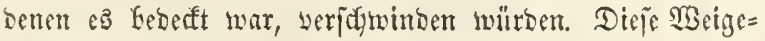
rung forten bem Sedjeif, ber auf biejen Jaengit nech weit mefir hieft, als er für gut befunben hatte, nir merfen

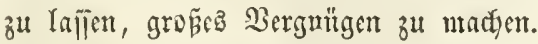

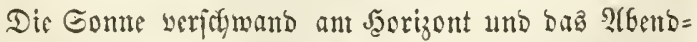

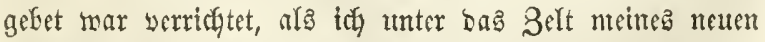

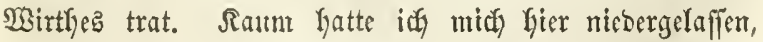

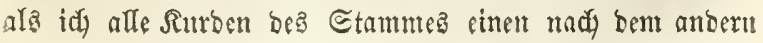




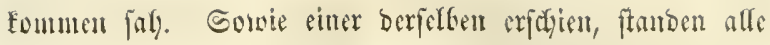
frillyer csingetretene auf und madtent eine grof̧e Berbeu=

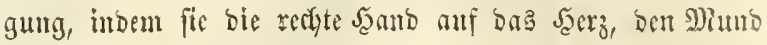
unb ben Sopf bradjen und folgente Sisorte fpradyen: Sebahh el qraï eich halack (guten Tag, wie befinden Sie fich?) Dicje mmftünolidge 2(rtigfeit ermente fid menig=

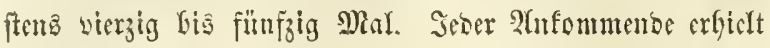

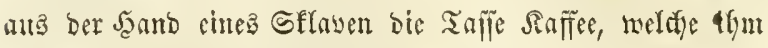

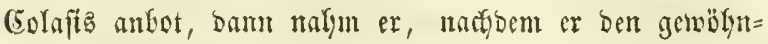

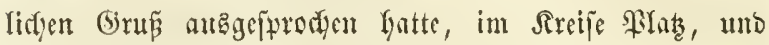
Das 3elt, fo gerïumig es auch mar, fap̃te faum bie $3 a b l$

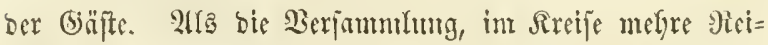
Gen bildonts, beifammen mar, wentote fith ber Soryeif zu

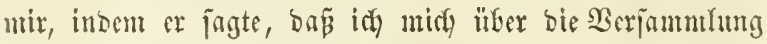

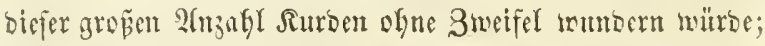

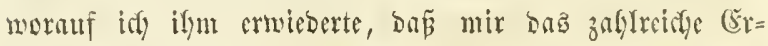

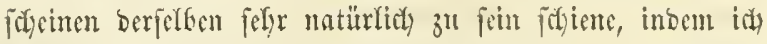

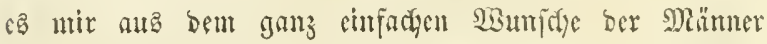

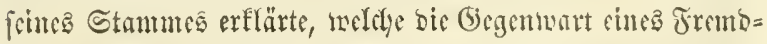
lings in bem geftfreumblidyen Belte ifyres Säuptling

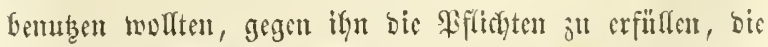

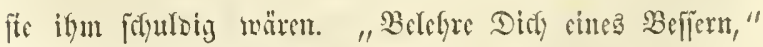

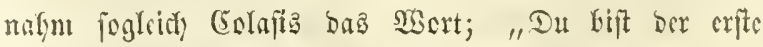
Jranzoje, melder in unferer Mitte erjobuen ift, und ift ciugig und allein, un Didy mohl zu emtrangen แnd

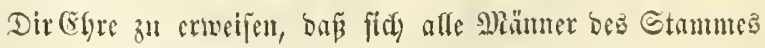
fo Eecilet faten, fids zu mir zu Eegeten." 
S(t) Dunfte bem Eityeif uno bat ifu, biejer jo zabl= reida verfammelten (bejellictaft, weldye midy umgab, meime

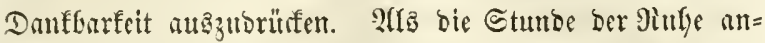
rïnte, begab fitc ein Jeber nad) Sauje; idy ftresfte midy uteen bent Gibeif alf cinen Tetpidy, ber mir als 9 ) $a=$ tratse biente amb faflief ein, nadyom id mir eines ber Siffen Dcs Divans ars Ropffijen untergefdyoben batte.

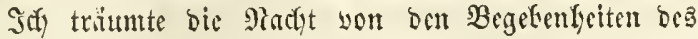
yorigen Tagę. 2 m Morgen bei Sonnenanfgang war ber ๔abeif foryon aufigeftamben. (5r Gatte bas geringite (5erianid) zu mad)en vermieben; aber mein Calaf mar fo Ycidyt, baj idy bei ber erften Berwegumb, bie er martyte, feinen Teppidy zu werlañen, amparde. (5r munfote mir

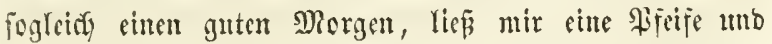
Rnfife bringen und fragte midf uatifger, ob idy mit ifm

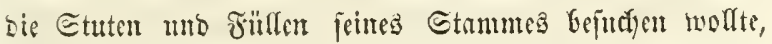
welcte alle anf einent nahen Berftenfelse meiocten. Diejer

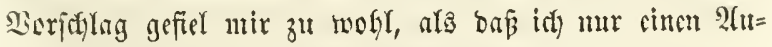
genbliff gätte jögern fömen, ifn anzunelynten. Soly ant=

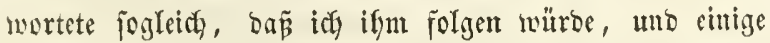

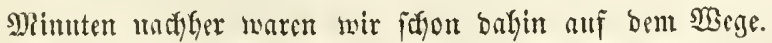

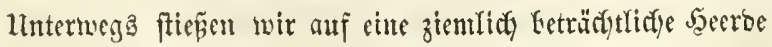

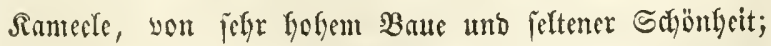
bicflben batten bei einent Belte ifren Stanbpunft, alle waren in eimen Sreis gefteflt, uno man batte ifren $\mathfrak{B} 0 \mathrm{r}=$

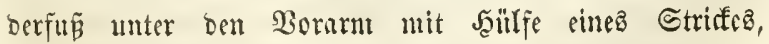

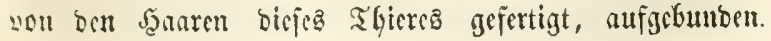




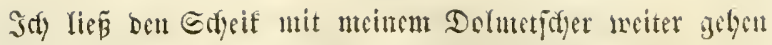
unto näherte mich biefer präatytigen Secroc, um bie ser=

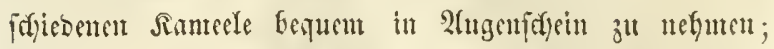
als id mun meinte Peugierbe wöllig befricbigt latte, cilte idi, Colafis und Steptens cinzufolen. Sie befanden fidi) gar nicht mefye weit won mir, als idf wor eittem bebent= tent grofen Bolte vorillerging, Deffen vberfte Stangen mir gewartjan erichütert zu meroen fodienen; ich görte cinen burdybringenden Ecyrei auz ber Seflye ciner Jrau,

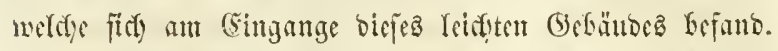

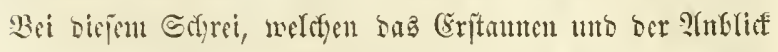

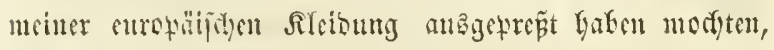

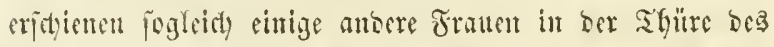
3eltes, weldye jut mir eiftent und midy tuntringten. Sinige

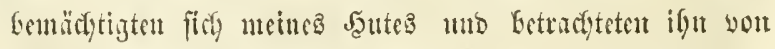
allen Sciten; anbere crgriffen meine fuprernen Mooffnüpfe

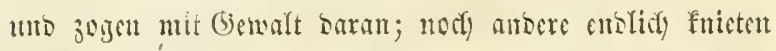

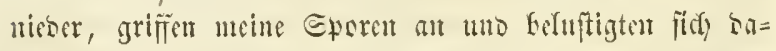

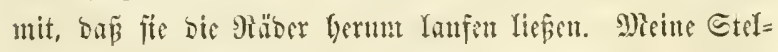

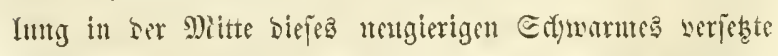
midy in cinige Berlegenlyeit, bem idf mar ïfer bie \$litter,

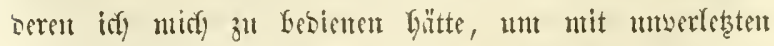

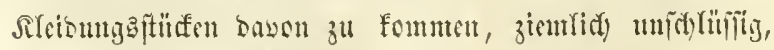

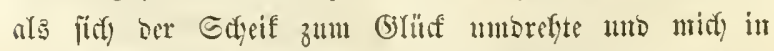
biejer fonoerbaren \&age jaf, in weldje midf meine Rlei=

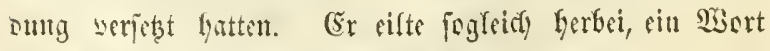

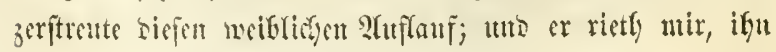




\section{1}

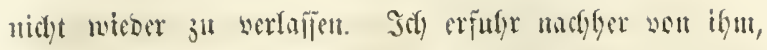

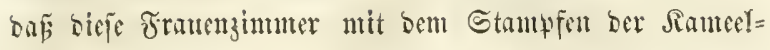
Gutter. Geichäftigt maren; fie erlyalten biejelbe, indem fie bie Milted biefer Thiere in Borffetle gieñen, bieje an bie

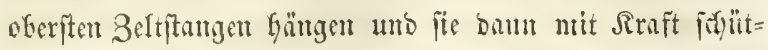
teln, fis fie bie wollfonmene Selyeibung ber Butter= jubjtanj you ser Bittermild jumege gefrudjt habent.

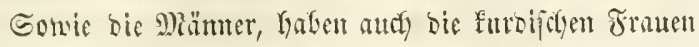

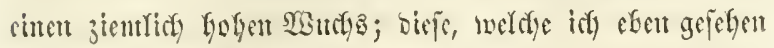

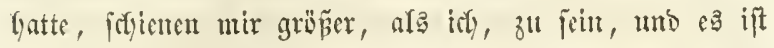

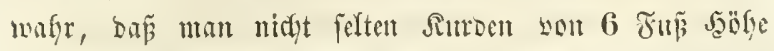

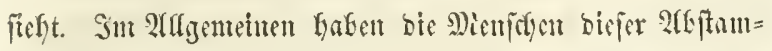

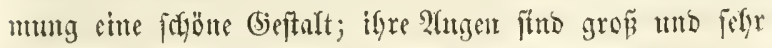

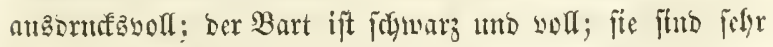

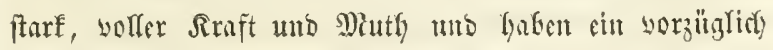
friegerifactes Temperament. SGre Rleibutng unteridgeibet fitr) menig yout berienigent ber Turfontanter, mur Einige tragen allf bem Sopfe cine Sitt eijerter Selme, weldte

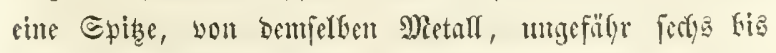
adyt Soll lang überragt.

Eolafis, Etephens mo ich, wir Gatten inderifen un=

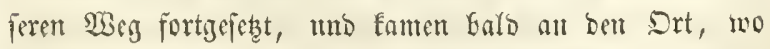
bie Stuten bes @tammes mit ifren Fünflen weibeten, dic alle won bom giferbe afjanumtem, won meldyem ich oben gefprodyen habe. 2(tle Butchtifuten gatten ftarfe Glicos=

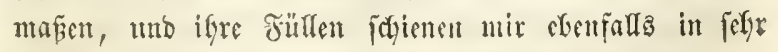
gittem ๔tanbe zit jeitr. 
2ha mente Srüfung becmbigt mar, gungen nir it

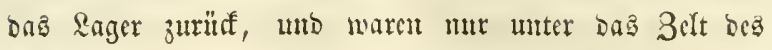
Colafiz getreten, alz or utir bas Jrülitüte anftragen

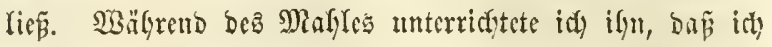
bic 2lefitgt bätte, morgen frïg abzureijen. Dicje siad)= ridyt fajen ifm reio zu thun, benn ex jparte Nidytz, um

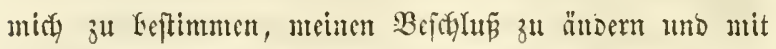
ilgm noch einige Tage länger teijummen zu bleiben; or ging bariu jo weit, baf or mir eine (5ajellenjago mit

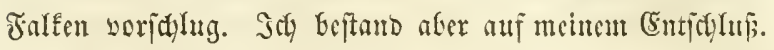
"TScil Du mid burfyaus verlañen willft," fagte mir Colaji: "To fammft Du gleidy Geute Deinen Fübrer, ben

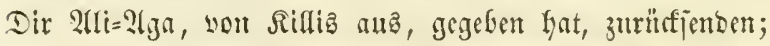

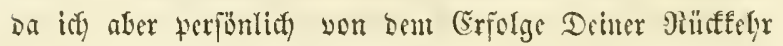

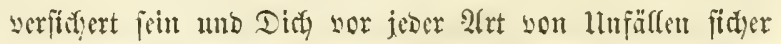
ftellen will, fo merbe idf) Dir zwei meiner Beiter mit=

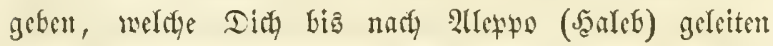
jollen; mit ifnen haft Du nidftz zu für(f)ten."

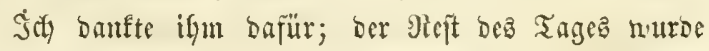
mit Spajiergängen um baz Rager verbrarift unto endigte

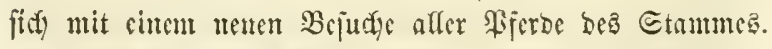
Gegen 2tbenb eridjienen abermals alle Rurben im Belte,

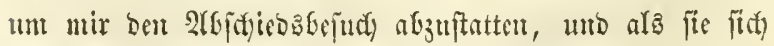
wicber entfernt Gatten, gab mir Golafiz zu micocrbolten

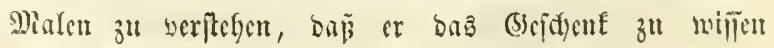

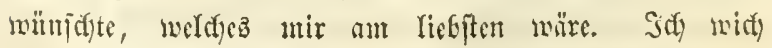
ben serferften Jrajen, bic er ẗber biejen Oegenftanto an 


\section{3}

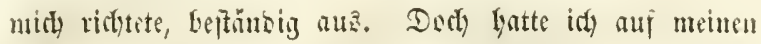
Epaziergängen mefree prärytige Sisinofyumbe von siner ber

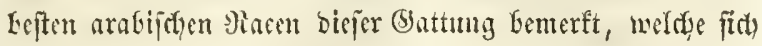
in ber gsinite auflyalten, unb latte gegen Etephens ben

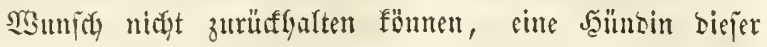

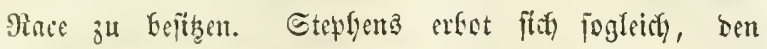

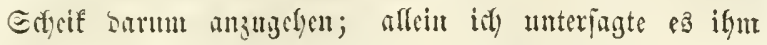

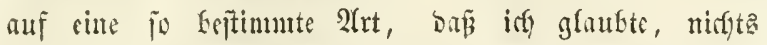
won jeiner zur Gemofntgeit geworbenten Pilauberfaftigfeit

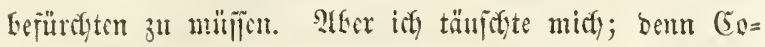

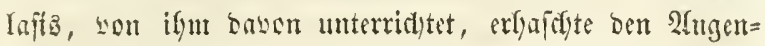
Gliff, wo ich eingeiftylafen mar, um an bie Watte, morauf

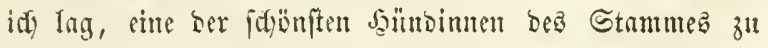

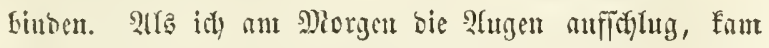

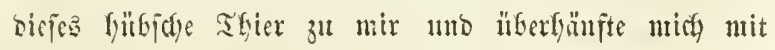

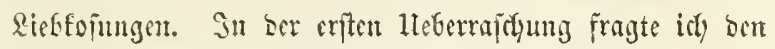

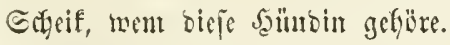

"Dir," antmortcte or mir, "menn fie Dir mefuntich

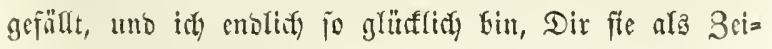

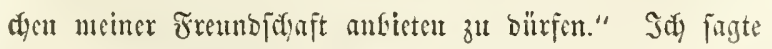

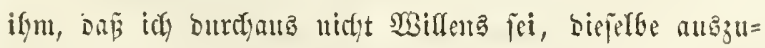
fafligen, unt kefragte ifn barauf nach bem ltriprunge mefrer senernarben, bie id an ben äuB̈ern umb untern

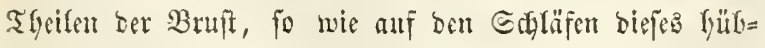

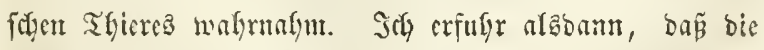
Narben folgenbe 3werfe ryätten: bie eriteren, um ifrr megr

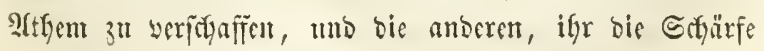




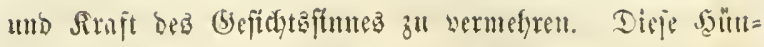
bin murbe in ber That als cime ber foundlften mb ffüg= fen ber ginfte angefefon. Edyon lange Beit war $5_{0}=$ Iaftis von Ruridjio=\$iajta um biejelbe angegangen wor= ben, batte fie ifm aber inmer wermeigert; or fagte mir

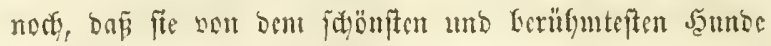

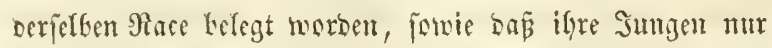

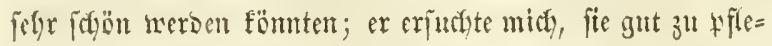

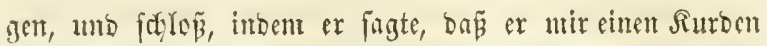

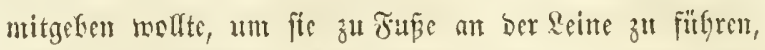

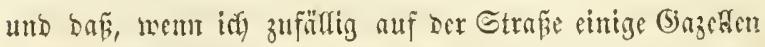

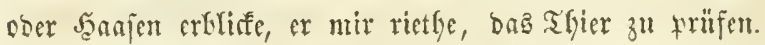

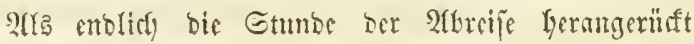

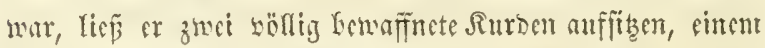
britten trug ef die Filfhrung Der Eingues (Name meiner

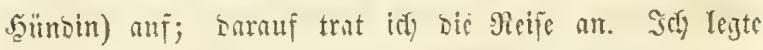
fic im Ecdritte mo ofue irgend cit merfmürbiges $\mathfrak{B}_{\ell=}$

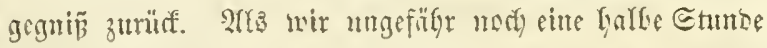
won Alefto entfernt waren, Gielten neine beion furben, meldye meine Bebecfum ansimadyten, an unb jogten mir, eấ ifuen die Befürrdtumb, won sen Türfen, ober ben

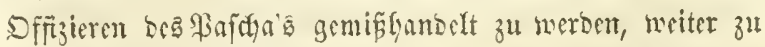
geben verficte. Eis münfixten mir sine gute Gejunbbeit uno waren im Begrifie, igre Siferbe nath ber Bidutung ibres sagers fortidyięen zu Yaj̃en, ars idy fie nody aufo lielt, um cinem jeben einen franijeden Siafter zu geben. Sie madyten jecod, Gdymierigfeiten, biefdlen amzunehmen, 


\section{(5)}

weil ifgnen, wie fie figten, Colnfis fereng verfoten hätte,

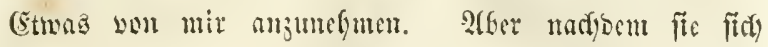
ein menig geftrüult, uno idy ifyen begreifich gemadyt fhatte, Don ja Colafis nie etwas yon biejer Freigetigfeit crfafyrem mürde, fyelten affe brei bic bände auf mb ver= idtwanden nadiger. Der Füfrer meiner Ginguès yatte

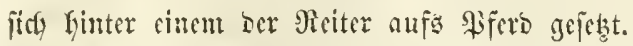

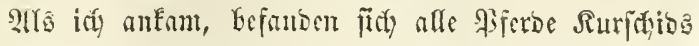

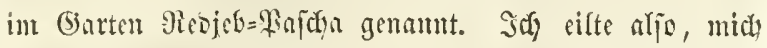

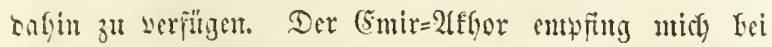

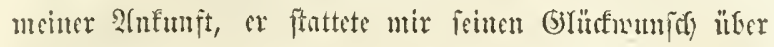

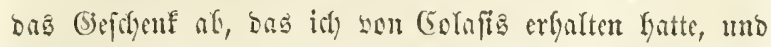
jagte mir, bos or fryon oft won ber Cimguè are ciner

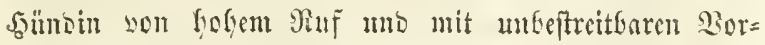
z̆igen begabt, babe jprecten bëren.

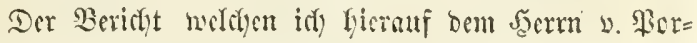

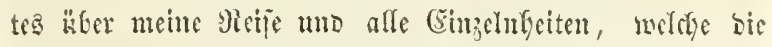
Dancr berielfen feffimmt fratten, abjtattete, feweg ifa, mit

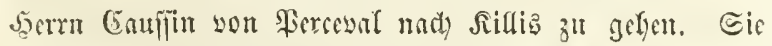
reiften mady einigen Tagen ab, unb Jeert you Forteß

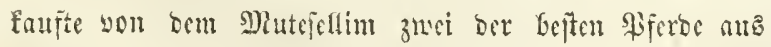

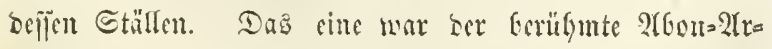

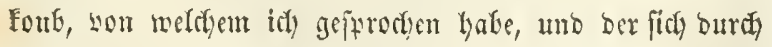
bie Etärfe und Ecfönffit feiner Eprunggelenfe bejonders

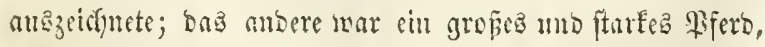

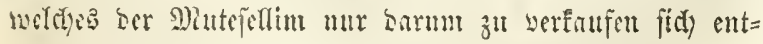

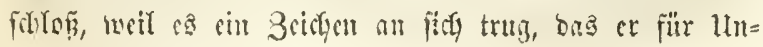




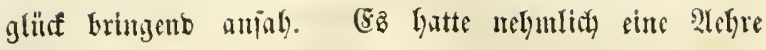

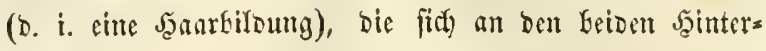
barfen Gefant. Danf bafyer ber abergläubifaden Jurdat,

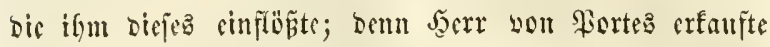

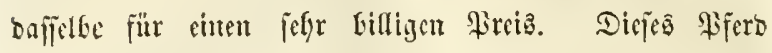

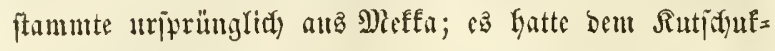

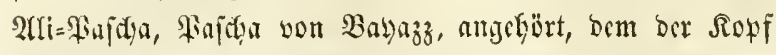
einige 3eit wor umferer 2(nfunjt afgeldonitten worben nar; nir gafon ifym Den Miamen Meffami.

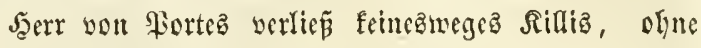

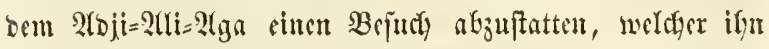
mit ber aufridjtigiten 2 (rt empeñng, und ber gegen ifhn

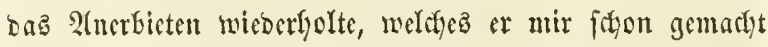
Yatte, Daz aber zumu gröften Errftaumen bez 2(bji nod) eimmal afgelefut wurbe. Derfilbe hatte nidyt genug 2 tubsbrürfe, bie leberrafajung z̆l malen, welthe ifgm eine für

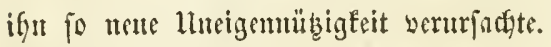

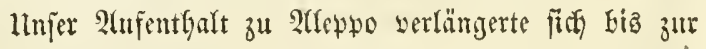

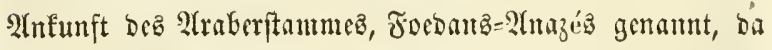

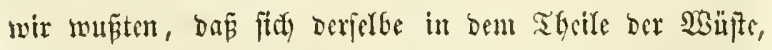

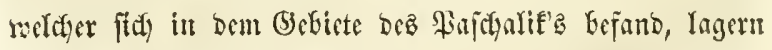

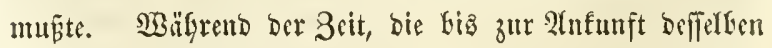
werifridy, hatte id Befegentheit, bas Dajein utb bic fon= Derfaren Eisgenfeiten ciner Gattung von Thieren za be=

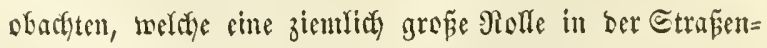

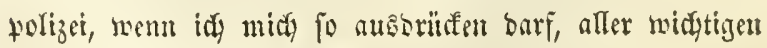
Stäbte bes Drients fficten. 
3" Slew中o, wie in allen Stäbten der Türfei, gitt

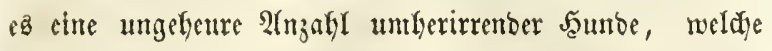
bie Etabtviertel, io zu jagen, unter fith theilen, in bent=

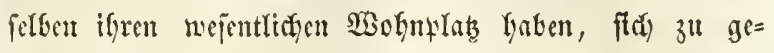
wifien Etumben unb an gervifien $\mathfrak{B r a ̈ z e n ~ v e r e i n i g e n ~ u n o ~}$

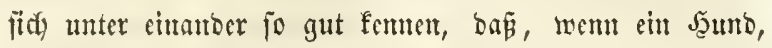
ber einem antoern Etabtviertel artgeförtt, bas llnglüf bat,

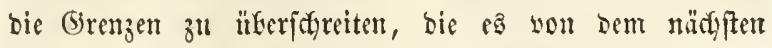
Biertel trennen, jogleid) alfe Benofiner befielfen über

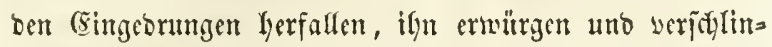

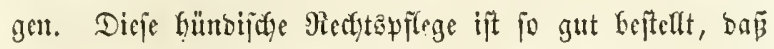
man biefelbe jeben Tag an aruen Trowfen aubälfen feefyt, meldye vor Samger fiterbento einzubringen nagen, um mit

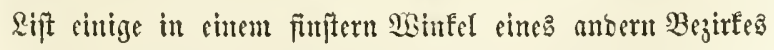
liegen gelaijerne Broffen zir Golen. Leferbier ift bie

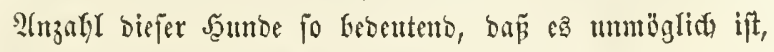
in ben (baffent einen Edyritt z̆ thun, ofye gantzen beer=

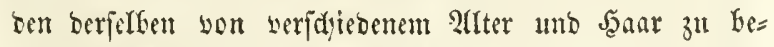

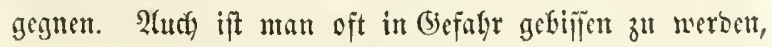

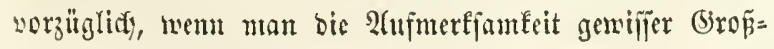

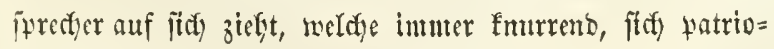

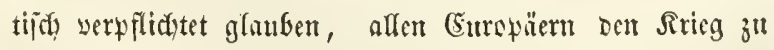

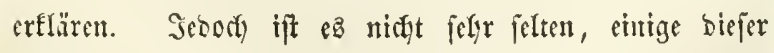

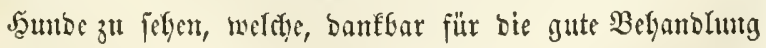

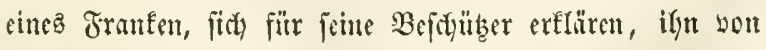
cimem Stabtviertel zum andern auz Danfbarfeit begleitent

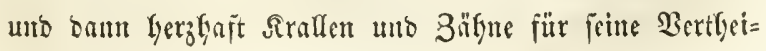




\section{8}

bigung gebrauden. Sedes Biertel befizzt mełre bergleiden

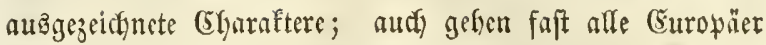

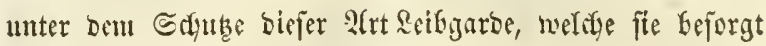

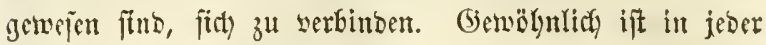
(Gajî cin Tüfe Genufragt, bicjen Thieren bie Nafrung

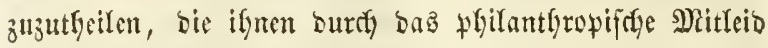
ciner grofen 3afyl treuter Diener bes Sropheten angerries jen miro. Diejentigen ber Syumbe, in beren Begirf bie

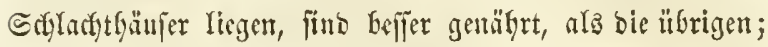

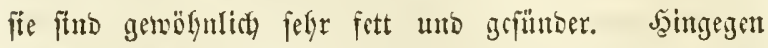
gleidyt Miduts ber Miagerfeit und Sodymadygeit berer, welde Dic Tobtenäfer Gemolynen, bie man an ben Thoren alier

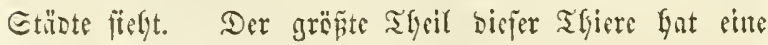

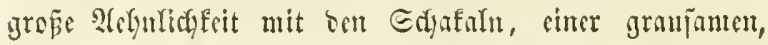

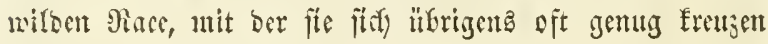
(wermiliftert).

Sit) Yabe misch nur besimegen bifer bieje notgmendi=

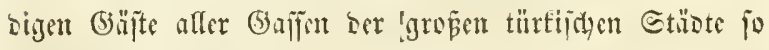

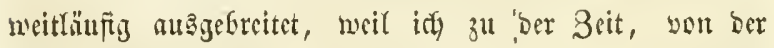

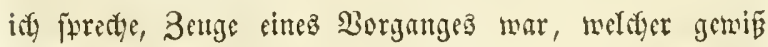
jeftr jouberbar ift.

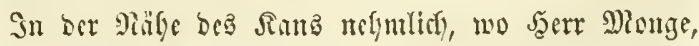

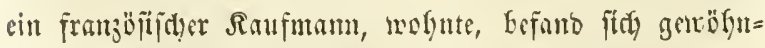
lich cin jefre jtarfer Saumb als Erţifbmadye, weldyer bejon=

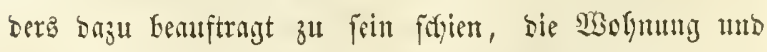
bie lungläubigen, bie jie cinjollon, zu beobarften. Die

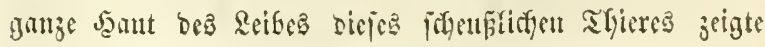




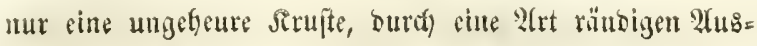

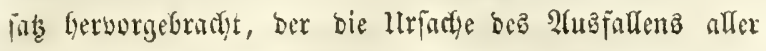
feiner Saare gewefar war. Heberblep beiping und bäs

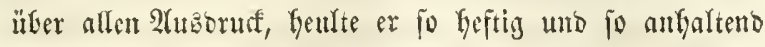

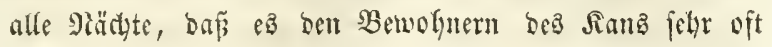

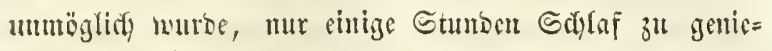
Eent. Sacre Mionge mar alfo ungeduloig, fitt) eincz fol=

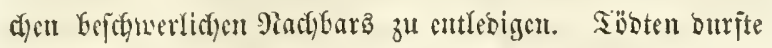

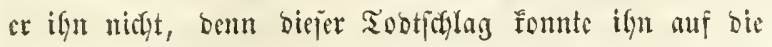
ernithaftejte Saseife mit ben Türfen in Etreit bringen.

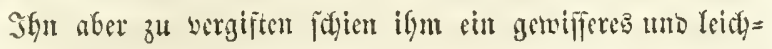
teres Mittel zul fein; er Gat mide baher, ifm bie Mittel

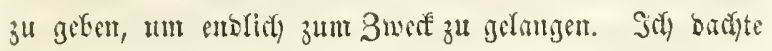

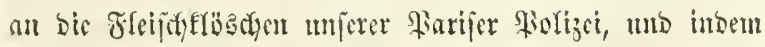

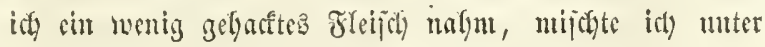

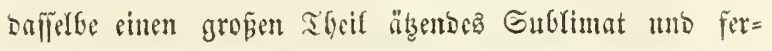
tigte cine Fiffe, fart gemug, um burrdy bie Dopis bitît,

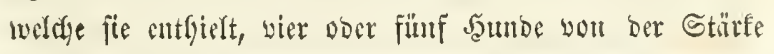

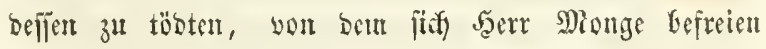

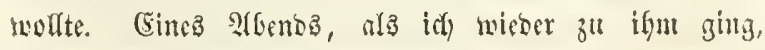

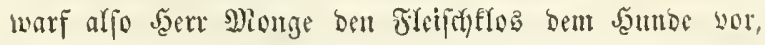

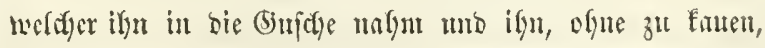
veridglang. Den andern Morgen glaubte ex fird won bem

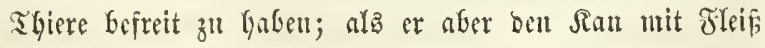

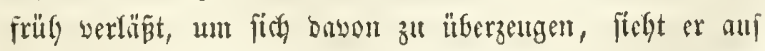
eimmal biefez fonberfare Thier auf fith zugetaufen fome

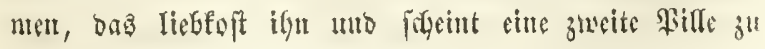




\section{0}

werlangen. Jatr Dionge beetle firt) nun pogleidy, mir

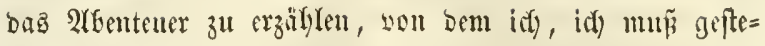
Geut, fefre ïberrafdyt murbe. Mgich barauf fogleidy wieber all Die Rrbeit begebend, werfertigte id) eine jueite Mirtur, iir weldye idy biefez Mial melyr alz wier Suentellen Subli= mat that, meldes gerabe fo viel mar, mu Damit bie Sallfte ber Sande in ber Etnot zu tösten. Sn feuter SBobmung angefommen, crueut 5ert M)ionge am Rlbeno Den Berjudy. Der Fleifdyflos wiro mit Det mefmlidyen

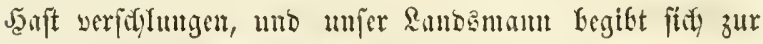

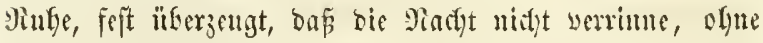

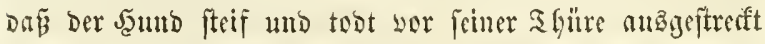

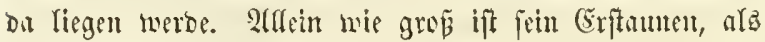

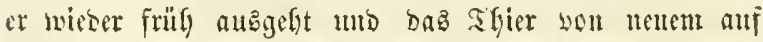
fid) zu foringen uno alle bie Riebfojungen miebergolen fitelyt, meldye er and ben vorigen Tag erbalten lyatte. D)it= leid erfülte fein Serz, er vergictstete auf bie Sergiftung uno gुab ben Befebl, bempelben alle Tage eitt mentig Jut= ter zut geben. Bon biejen Rugenbliaf aut Görte biefer

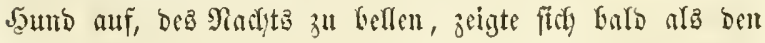
wärmiten Freutb afler (suropäer, nnd was not) fonoer= barer ift: er cin yrädtiges ? ficr.

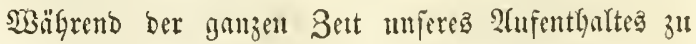

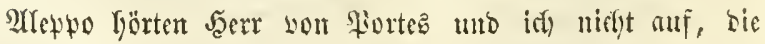

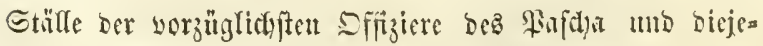

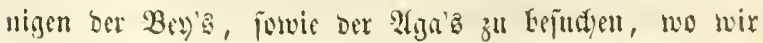




\section{1}

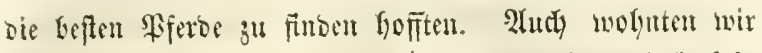

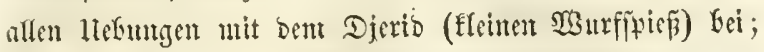

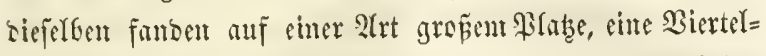

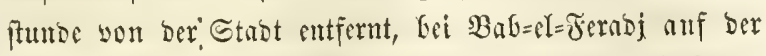

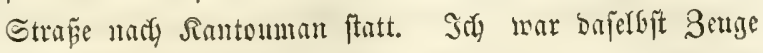

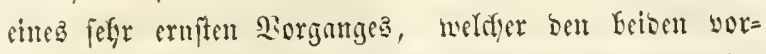
nefymiten Theilnefyutern biejes Rampfes bob Refen fuften Eurmte.

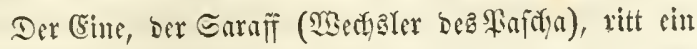

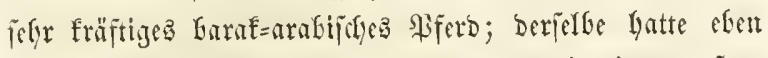

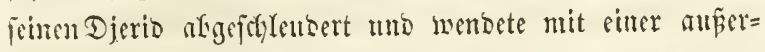

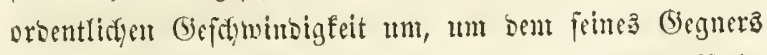

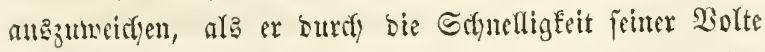

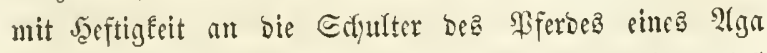

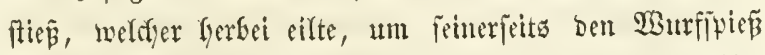

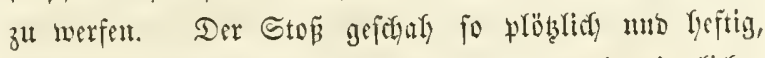

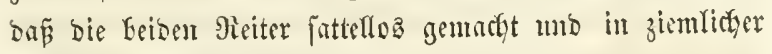
Entfermurg von ifren ßferben abgemorfen murber. Der Boben war mit fleimen Riejelfteinen bebeft, unb ba ber

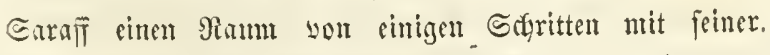
๔tirn uno Bruft fo zu fagen aufgemulthlt hatte, ftand or

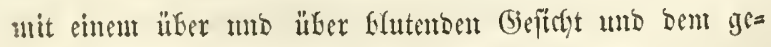

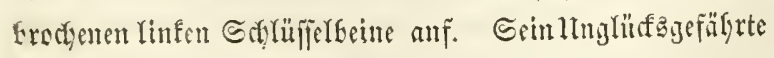
murbe meniger bejçäsigt, er fam mit cinigen wenig ge=

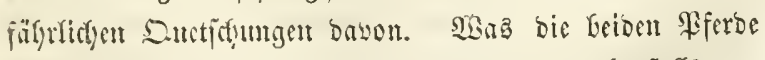
Fetraf, io murbe bas cine wie dab andere ebenfalla zur (Srbe getworfent; Der Stop, meldyen bie redfte Schulter 


\section{2}

oํี einen, und bie linfe bes anbern erlitt, verrenfte bei beiben bas Gর্ultergelenf.

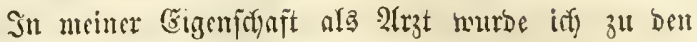

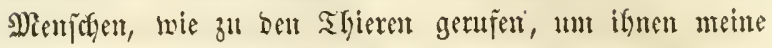
Sräfte zu mibuten. Das meifte Butrauen jdentte ntit

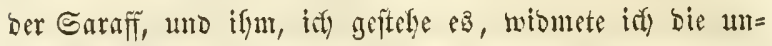

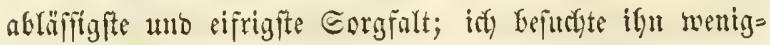

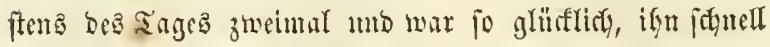

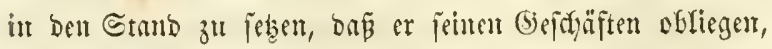
aber bock nict) reiten fonnte. Simutal zat biejem Sirad yon Benepung gefoumen, gab er mir beiranfig zu ver=

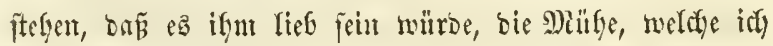
mir trimetwegen gegeben, burd) ein Battis vergelten zu

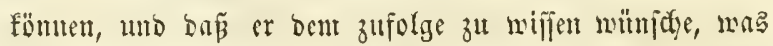
mir ant meiften geffele. Sit) batte feine Ställe bejudat unb barin unr ein 8 fiferb gefunden, weldes werth genejen wäre, won 5erm son Forteह umb nir gefanft zut werben;

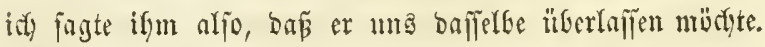

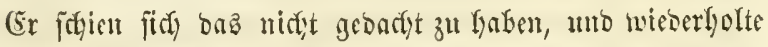

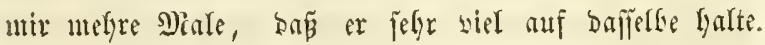
Ëunige Tinge barauf fofufte or mir als Bactitis eimen

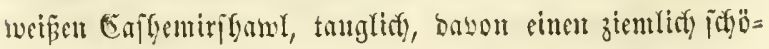
nen Turfan zu fertigen; Dann einige Beit nadyer, nady=

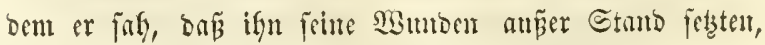

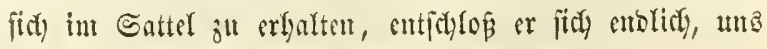

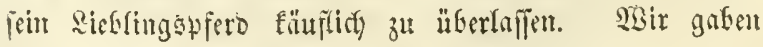

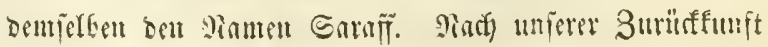


in Jranfreich fan biej̄er Jacngit zแn Depot nach \&angonnet, powie and ber. Nheffami, eit anderer Jaengft, welifyer won

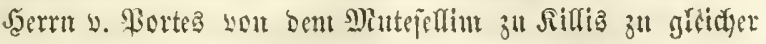
Beit mit bent 2 f6ou=2(rfoul gefunft morben war. Der

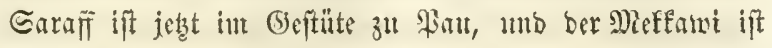
ju Santignnet gefturbent.

Biz jest fyate ich ju ermäfmen verge

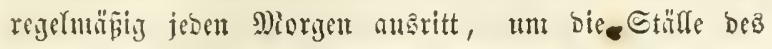

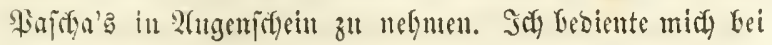

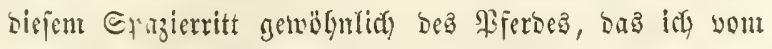

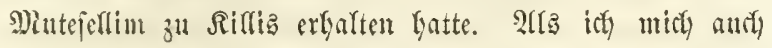

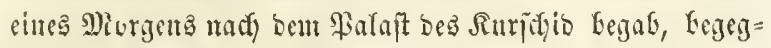

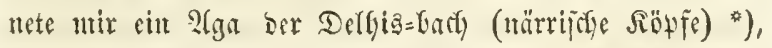

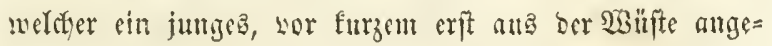

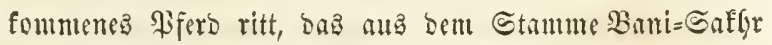
fam. Sogleidf fetr mager, froien ntir bort) bas Thier

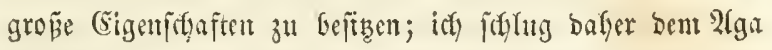

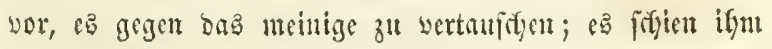
jebod nidyta baran za Yiegen, bent wir trennten ung,

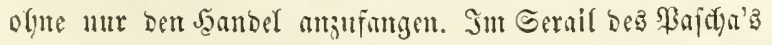
angefoumen, that idy nidhts eiliger, als bem (suir=2(ffor bie Sacje ntit bent 2 ga zu erzählen uno ifut ben you

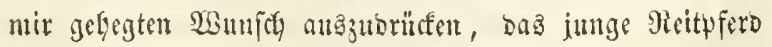

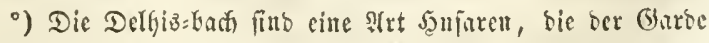
ses अajha's einverteist jino; ifr Ropf ift mit einer langen

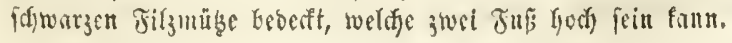




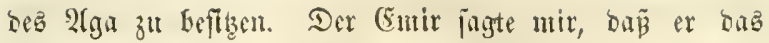
Thier fefr gat feme, und mas nody mefyr ju bedenten

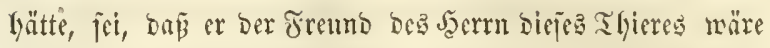

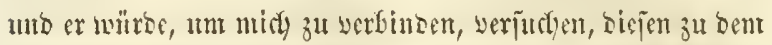

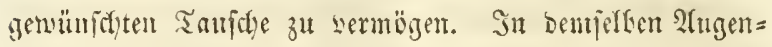
blicfe, als er mir feine Dimftantervietnngen ntadyte, mollte

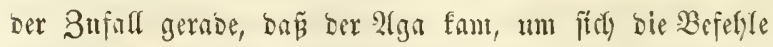

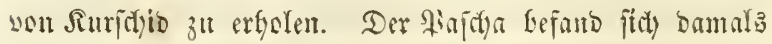
utit jeitent ganzen Sofe in cineut (Sarten, weldyer unter

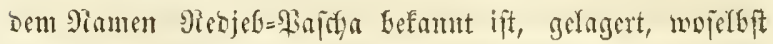
fich aud alle feine Prerbe auf Den SBiejen befanden. Der (5nir frrang fogleict) zu bem 2ha mo lasete ify ein, in feinem Bolte Rã̃ee zu trinfen; sarauj ffrady or yon

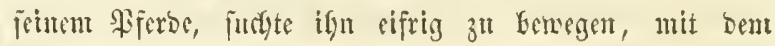

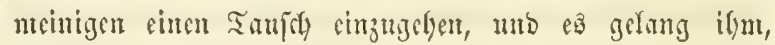
Denjelben bazt zu beftimmen, indem ich 80 Piafter ferats=

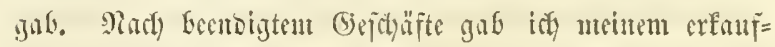

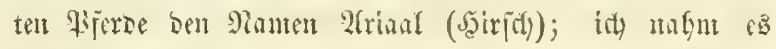

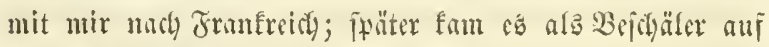
die Snipl Diartiniqute.

SBälyreno fidd ber 2lga nit mir berumeftritt, wari ber (Smir lïpterne Blife auf einen englijdyen Sattel, ben inf son Paris mitgebracidt batte; er forady fith barnber

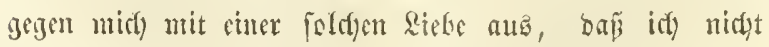
umfin founte, ify benfelben anzulicten. 2(ud) nabm er if) zu mteitem grof́en Reiomejen an, ba

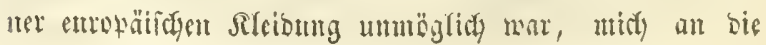




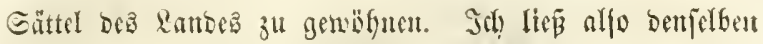
jul ifur tragen; alfein ben folgentoen Tag fractete $i$ gan ber

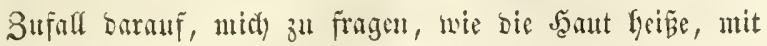

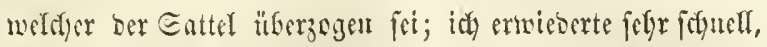

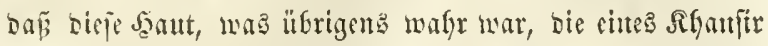

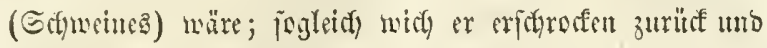

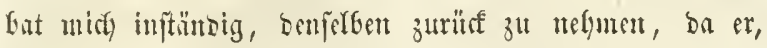

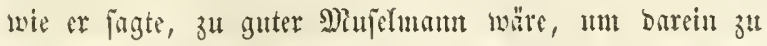

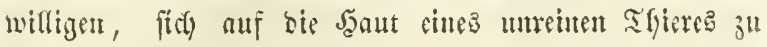

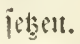




\section{3ites Sapitel.}

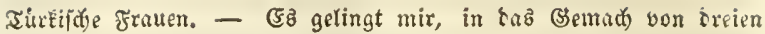
terfelben einzutringen. - 2unfauf teb నatmor, tes Srcan un

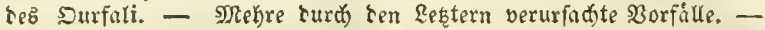

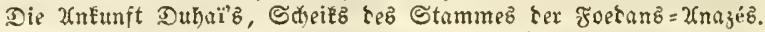
- Er i - 26reife von Jreppo in tie Srzúfte. - Die Douaiq (Juaik). -

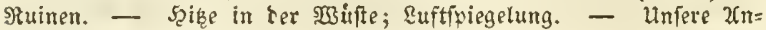
Eunit zu Tel=el=Sultani. - Durdizug piner Raramane. - $\mathfrak{U n}=$ Eunft Ser Foctanz=22najés; fie fdragen ibre 3elte nuf; betontere umftản hierbei. - Sffenberjigkeit รeb Dubaï; Nittrgêmahl;

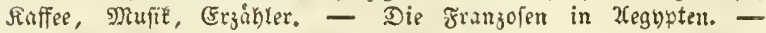

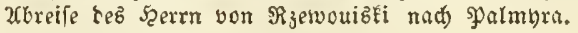

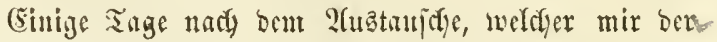

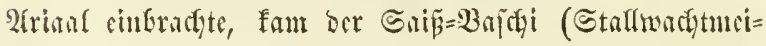

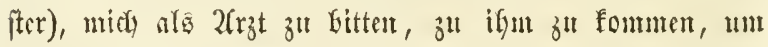

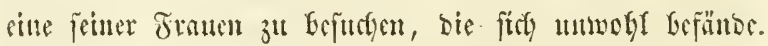
Sit) nufut bas nit bent rebrafteften Sntereffe an, in ber

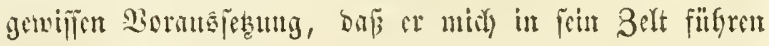

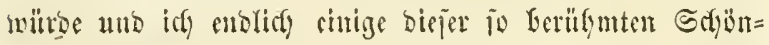
beiten, welege bie Tünfon aus Georgien bejieflen, whtrbe

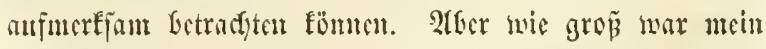

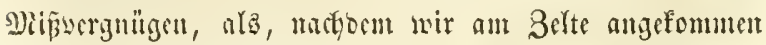

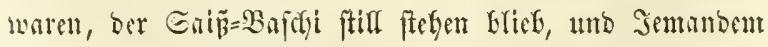
iu Suncrn bes Belte folgenoe Worte zurief: "\$ier ift bor

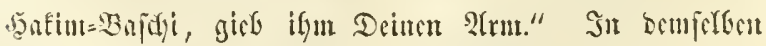

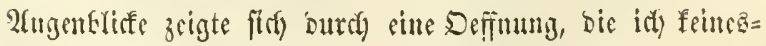

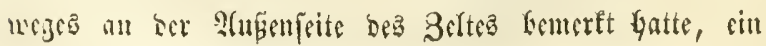




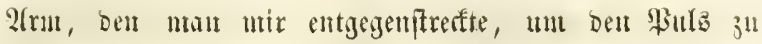
unterjucfon. Sory befïflte ifyt mo jagte darauf dent Saíf, daf idy nidyt bas geringite Gintadyten undy einen fo

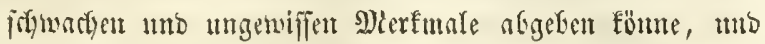

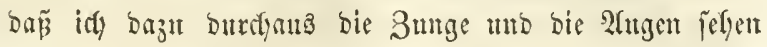
mü̈rte. Die Sranfe näferte fich Daratif, anf Den Bejefl

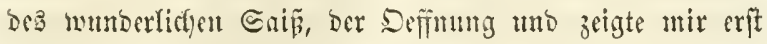
die Alugen, Dant die 3unge. Dieje tgeilweipe und ver=

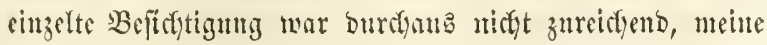
geftige Nettgierbe zu befriebigen; idg jagte aljo bem હaip

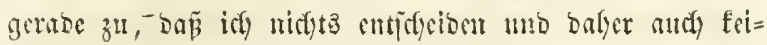
men $\mathcal{A}(1$ हี

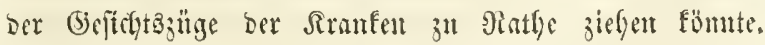
Dieje neue Forberming jolen ifgu zu criftreften; er jab

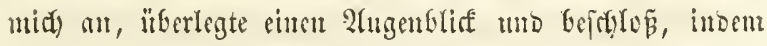
or anderen Frauen ben Befegl gaf, aber ummer surfy bie

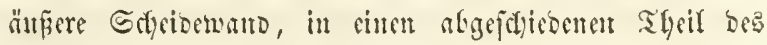

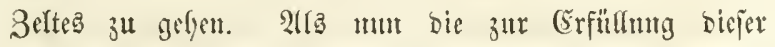

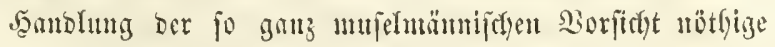
Beit werftricyen war, trat idf entidich unter bas fo $\mathrm{cr}=$

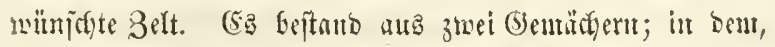

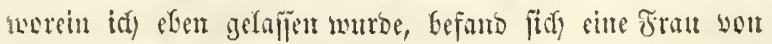

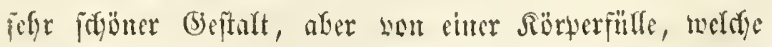

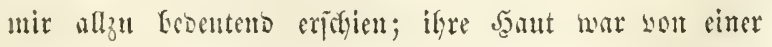

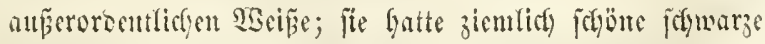
2lugen, cine birfe, furze Slafe, fomie birfe, blangenalte

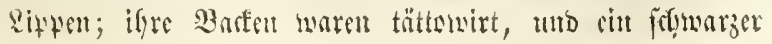


Streifen mit Shoel *) gezetrfutet, mugab ifye :Hgen; ibre

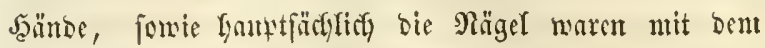
Safte ciner Pffanze gefärbt, bie fie Semmé nentem, melifye fite ans Regyten bezieben, unb sie cine Mafagonifarte gibt. Dieje Jran mar bie Sirante. $2(13$ idy jie mit Corgfalt unterputyt unb fie mir anf melyrere Fragen geantmortet batte, Die ifif rifer ifren 3uftand uno bie Edymerzen, bie jie entwand, an fite rictutete, glanbte idy fuct ifgr bie Symptome eiter angefenten Sitmangeridaft wafrgenom= nen ju baben. Saum hatte idj ifren Manm mit biejer Entoeffung befannt gemadyt, als er in jeiner Frentbe zmei antore Frauen berbet rief und midy Gefragte, of and bei

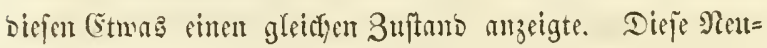
angefommenen Gatten bemalte Gefichter, wie bie Stanfe, unb ifre Sänoe waxen ebenfalls mit eimer 9lahagont= farbe gefärbt. Eine yon ifnen war eine 2trmenierit, weldye mir unbeftritten weit Gübjoger vorfam, als i⿹re

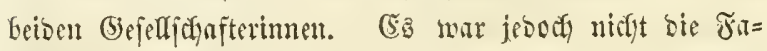
worite, bent ber Sranfen gebörte Der gröbte Theil, Der

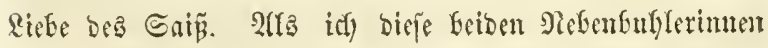

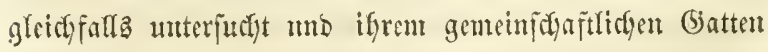

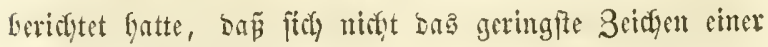
Edymangerịaft fünde, entliç or midy, insent or mis fagte, ba

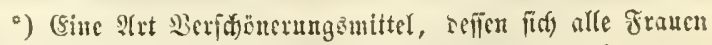

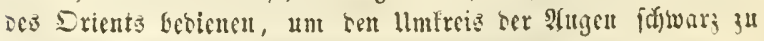
Finten. 
meue Diente uöthig bätte, reduste; allein or madyte midy jugleid verbintlidy, mide nidyt effer bei ifym zu zeigen, als bis or midy wïroe rufen laffen. Diejer Bejud war in ber That ber einzige, ben id ifyut madjte; idy faf ifyn

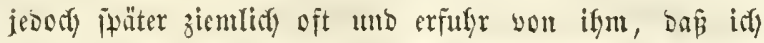

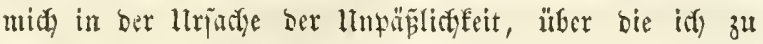
Rathe gejogen worben mar, burfiauz nidat getäujat batte.

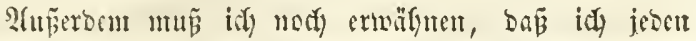
Tag gerufat wurbe, meine Diente netten siranfen zu wionten: Tünfen, Juben uno Ratgolifen müroigten mids

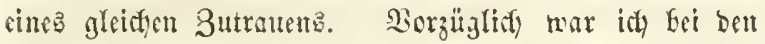

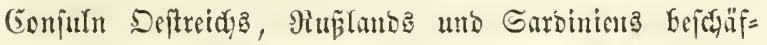

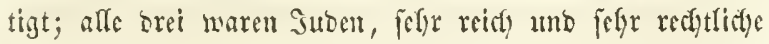

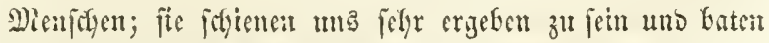

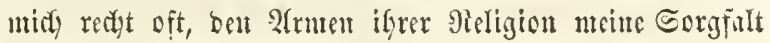
on fictenfen.

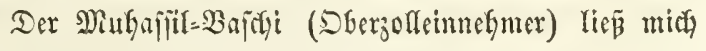
jefre oft bitten, feine Pjerbe zu Gejuchen. Şäbrend

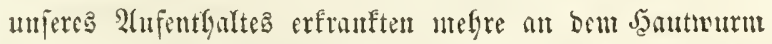
(Seradja), mo es gelang nir, fie in furger Beit Gerju=

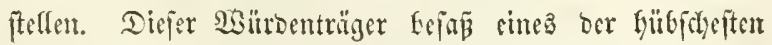

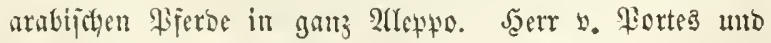
idy Eegelgrten baffelbe zu faufen, er nber weigerte fid lange Beit und willigte erif priter, cinige Tage vor un=

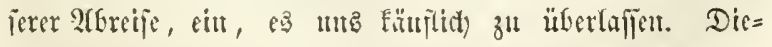

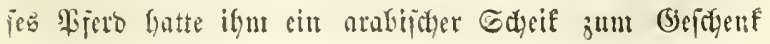




\section{0}

gentad)t, beffen Belte bei ben Mininen bou Siafmyra auj=

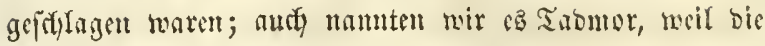
Traber biejen sianen ber fo beriffmten Stabt beilegen. Itm e⿻ ju erbalten, maren wir aber genöthigt, jugleidy ein altoeres Prferb, Drean genaunt, mit zu faufen, beffen

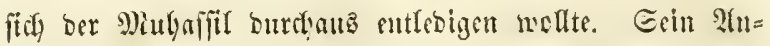

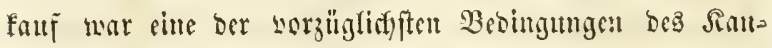

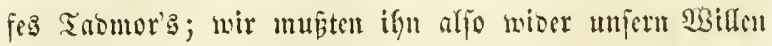
mitnebmen. Der Tabmor mar bas Paferd, weleyes der

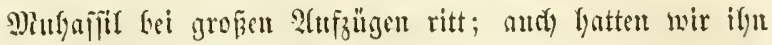
bas crfte Mial, mit bent reichfiten Ectjmut beberft, in ci=

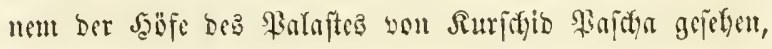

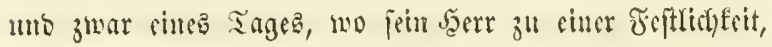

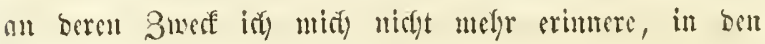
Esrail gerufen morben war.

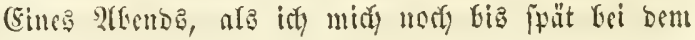

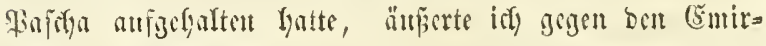

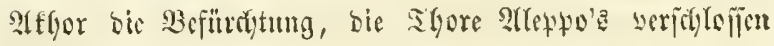

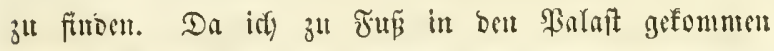
war, bot mir ber (Emir pogleict) ciur Pferb an, intom er mir jugte, bar, wemm ing ben biang beffelten becilen mutrbe, idi) 1tody zut recifter Beit in ber Stabt eintreffen

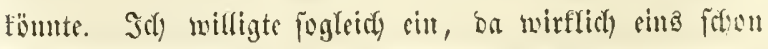

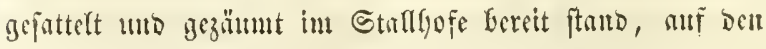
Jall, bá Seman feiner besürfte. Den ganzen Tag

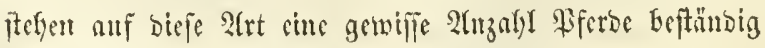

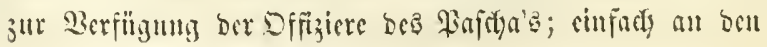




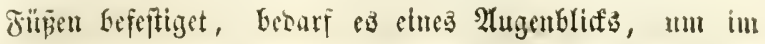

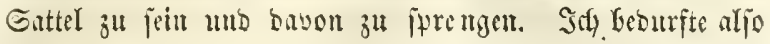
werniger, beun ciner SPimute, um auf bent gasege nady

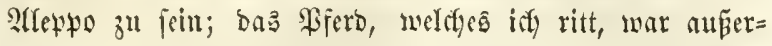

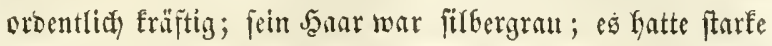

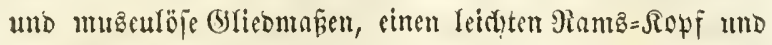

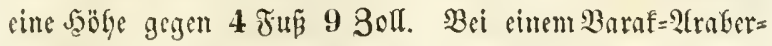
Etammte geforen, ber gembifnlich bei Durfa, auf ber andern

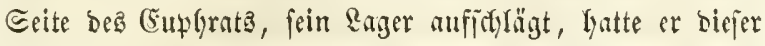
2rgftanunumg megen ben Namen Durfali erlalten. Dant

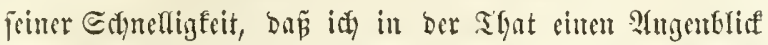

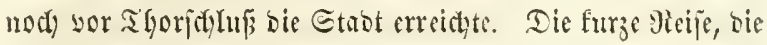
idf efen mit ifgu genadgt hatte, ferste mich in ben Eteno, Das Thier ju müroigen: idf fant in ifgm bas gröpte Sermb̈gert; fein Trab Gatte inesejonbere eime auperorountlidye Edguelligfeit, ofgletid) or nidgt an bieje (Bonrgart gemöbnt morben war, benn bie $\mathfrak{A}$ (raber unt Türfen, wie man

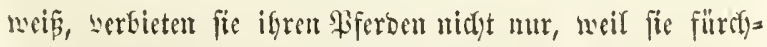

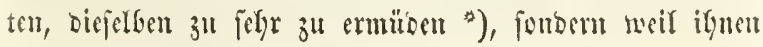

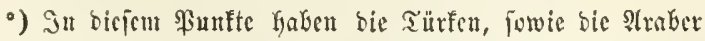
rine ganj falide ?ajicht, beun ber Balopp ift gerabe sic an=

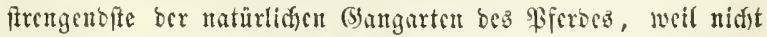
nur tie Stthmungarwerf zeuge melyr angegreffen weroen, afs im

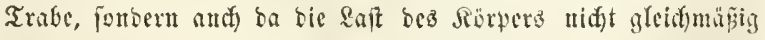

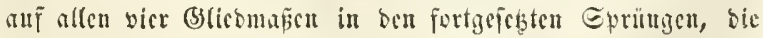
in srei Tempos erfelgeu, wertgeilt ift; ittocm fetz ber anpere

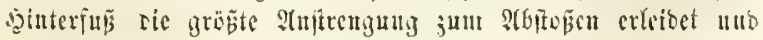




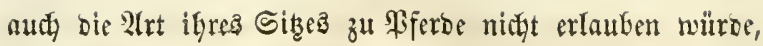

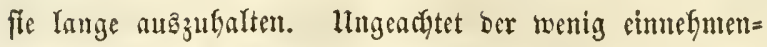
ben Form feines Sopfea, rieth itf bennody bem Stall=

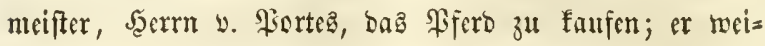
gerte fich feinesmegs, allein ex wollte baffelbe vorfer felbft verjucten. Mit ben Proben, benen er ez unterwarf, zu= frieben geftellt, unterhanbelte er Ḧber ben Freis mit Dem Émir unb fam in ben פejits beffelben. Der Durfali hatte cinen ziemlid bömilligen Gharafter; er liebte nicat, feimen Sillen unterjuoronen, unb bennod mar er leidyt genug zul reiten, wenn man ifn mit Befutfamfeit und (5)ïte Gebandelte. AGer eines Tages, no wir in Bejell= jefaft unit einigen Curopäern bie \$artfie weramfaltet bat= ten, Den Reif (Ruftritt) in eiment Der (Särten bei bent

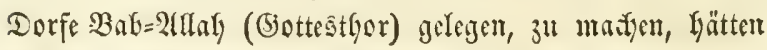

auf รem ăแ (Salcppipunge laftet; bie inneren Füpe fiłh hingegen freicr unb erhabener bewegen, fo werben aud jene baburd mefir anges

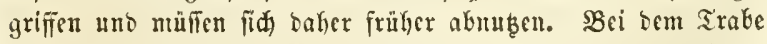
hingegen, welder in zwei 3eitabidutitten gefdicht, übernefimen

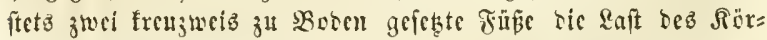
wers, wâfrend fidy bie antern beiben forjureitent frei über bem 3oben erlyeten.

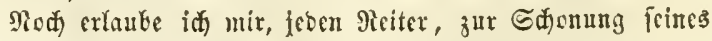

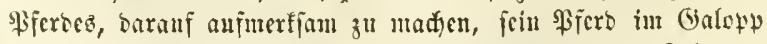
mandymal zu medffeln, Santit nidjt bie Fü felgr altgeitrengt werien.

Inmerfung bes lleberiezers. 
uir beinatse on 3ttranen, weldtese wir bismeilen ju bem guten Saturell bes Surfali Gatten, felye theuer bejablen mülfen. Die Gacte war nelyulict) folgenoe:

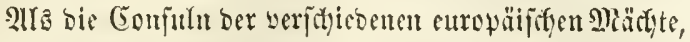
eine jienlidy grofie 2 magh oranfen uno igre Frauen fdion ineggeritten maren, jebten ficf) bie Secren (Suts, ע. Fortes uno Cauffitt y. Fercewal mit mix auf, um fie cinzufolen. Der Ctalfmeifter 2. Portez ritt sen Surfali, bie Serren

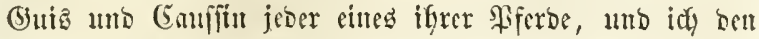

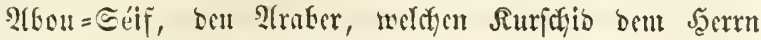

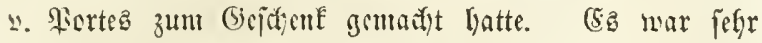
bein, unb wir ritten Edyritt; ars wir auf bent Blake

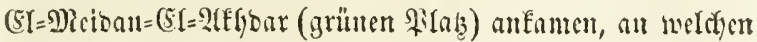

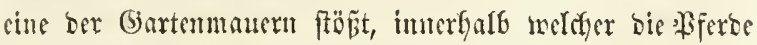

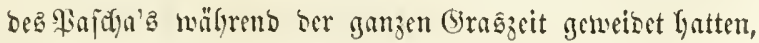

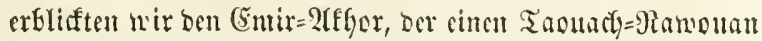

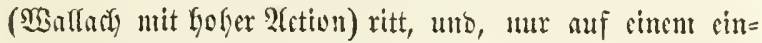

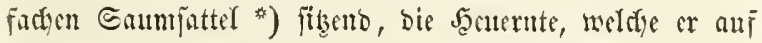

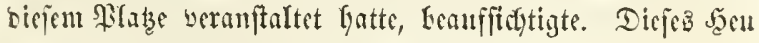
war bas erfte, Welcfes man in bicjem Ranbe einerntete, ba die $\mathfrak{B}$ cwolyuer ocffelfen fein anderca Futter für ifre \$ferbe Eennen, als zerriebenes (Serftenftrof unt Gierften= söner; bas Jeut war reifflidy unb gut. Eo nic ber (Emir un jofy, zog er ben હäbel unb ftürzte im Sajerz

-) Dic Tacnathi=9amouan tragen nic Sättel, fo wie sisicnigen ber (Gridge uno tic sisalfatyen. 
กแ1 แta zu; Serr won Fortes war ber Erfte, ben er er= reidute. Der Surfali, über biejen 2fnlauf in fordnellem

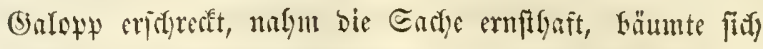
gejen feimen worigen Serm unb weriegte ifgm won worn

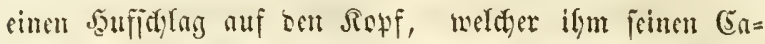
Gouf *) tief üfer bas (jefteft bis an bie Erfultern nic= Derstüfte; fei bentelben Efrunge Eradute or audf feincm

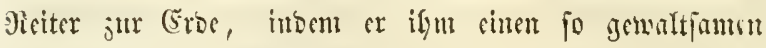

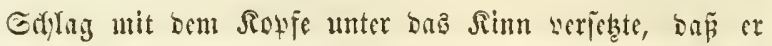
ifyn weit uno fait ofune Bemuffecin won firt warf. In

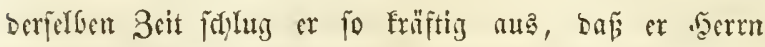
5. Siorte anjmeifelfaft getöbtet gaben würoe, wem ex surch ben @eturz minder betäubt gemejen wäre mo das

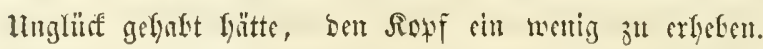
Somie fich ber Durfali frei fïflte, lief er nach sent Pferbe

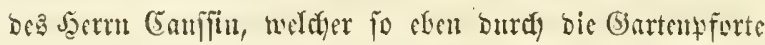
fu entoumen jutyte, or golte ign aber bei bent Exring= brunnen ein, fiel liber ifn ber mb verjetste ifum einen fo

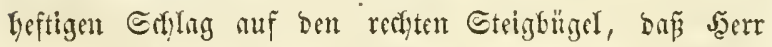
Canfin an Beine cine bebcutende Befdäbigung bavon

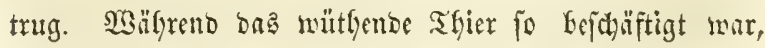

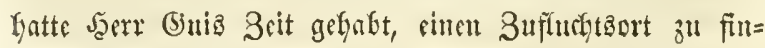
ben, uno ber Enir Gatte unterocfien feinen Ealyouf wieser

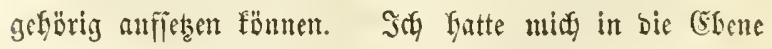

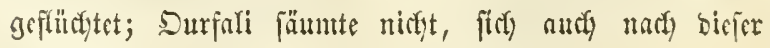

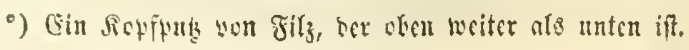




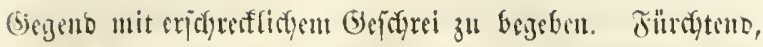
Dan er autr) anf midly fpringen mürbe, fprang irty in aller

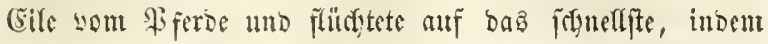

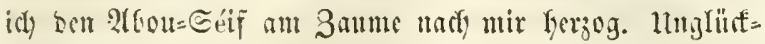

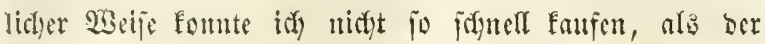
Durfali; er erreichte mich aljo bald, warf fted auf beu

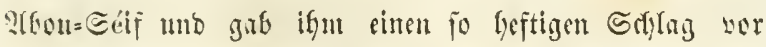

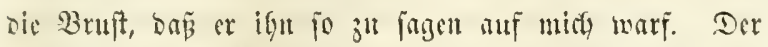

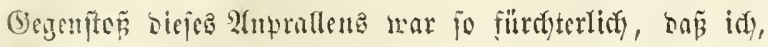
sinige Gedritte weit gefdyleubert, bie Eroe ntit uteinem Gepitryte, ber Bruft und bemt lluterleife aufitiés uno cinige 2lugenbliffe liegen Elieb, ehe int wieber zum Bemufticin

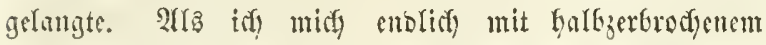

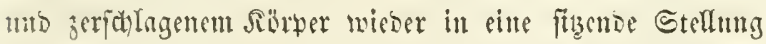

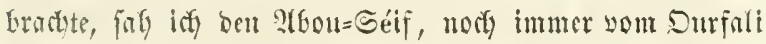
verfolgt, cine feltene Gatuelligfeit entfaltent.

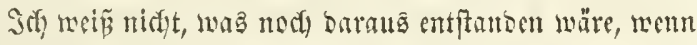

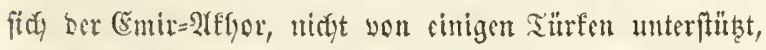

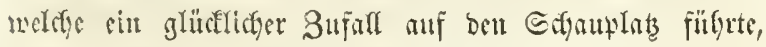
bemübt Gätte, bieje beiben Sämpfer ju fangen uno แn

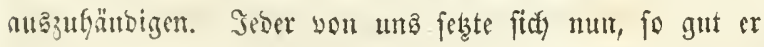
Fonnte, auf, und wir erreirfiten enblidy uniere Bejellidgaft.

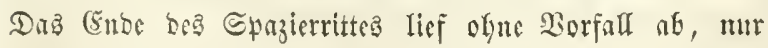

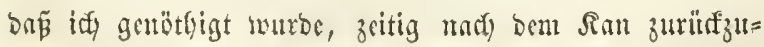
reiten, um für midy felfift Gorge zu trigen. Jah fam mit einer 2frt wou Gtrifgcit bavon, bie nath einigen

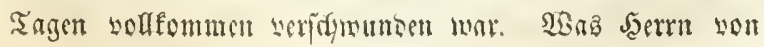




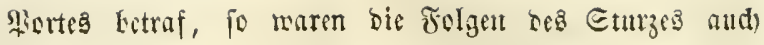

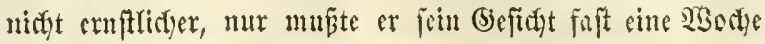
lang verbinten.

Dic Bcit Fan emblick Geran, wo mir ben Bebuninen= ftanm ferlen follten, won weldyem wir fidgon fo lange Seit

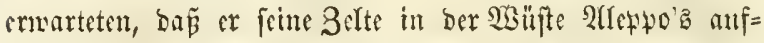
forlagen mürbe. (Ës hieft fich in ber Stast ein Janit=

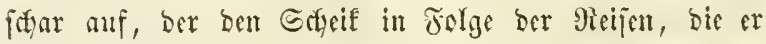
alfe Safree mit ben Turfomanen bortfin machte, auf bos genaurefte famnte, lweldye fith zu genitjert Beiten in bie

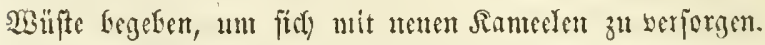

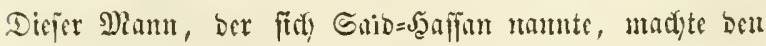
Miaffer in Bejiefyung anf alle Säure biefer Gattung; Ritemats als er, war aljo beffer won aflen Bestegumgen

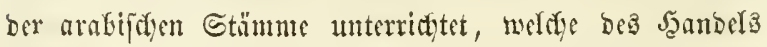

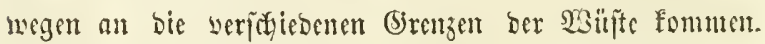

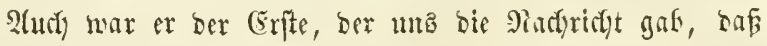

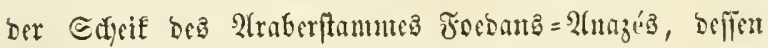

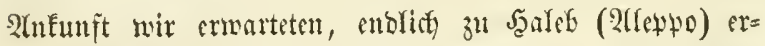

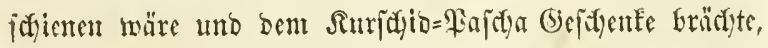

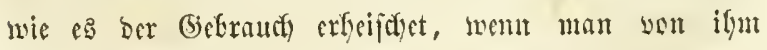

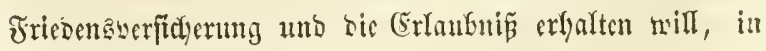

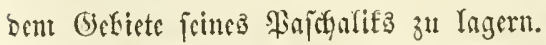

(b)eity bert folgenben Tag ging ber eble Fole, won

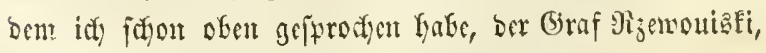

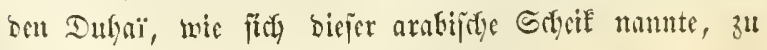

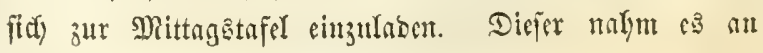




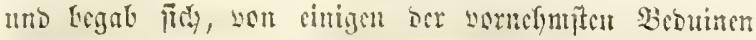

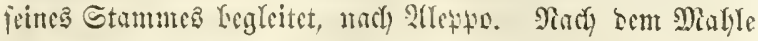
madite ber Graf feitem (5afte cinige Gejchenfe mo unter= fyandefte mit ifyn um ben Edyub, befien or bei ciner Meeife,

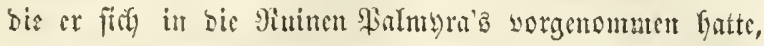

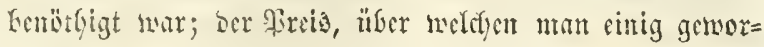
ben, war cin Sameel.

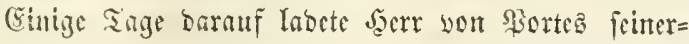

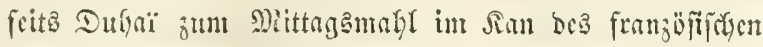

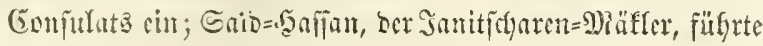

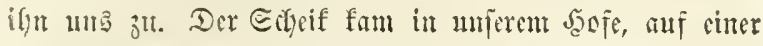

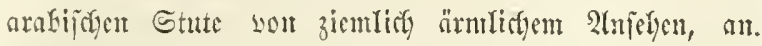

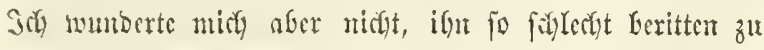

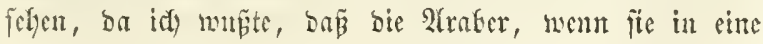

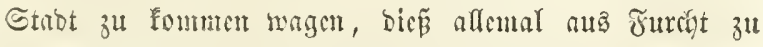

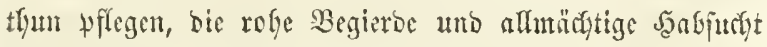
ber Türfen zu reizen. Duthaï nutrbe yon feitem bec Geimictyreiber Daub nud vier anderen Bebienten begleitet;

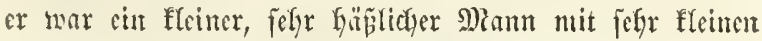
2lugen uno ber verbramiten Befichtafarbe ber Bemofiner

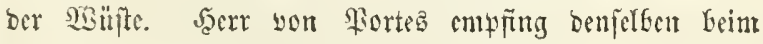

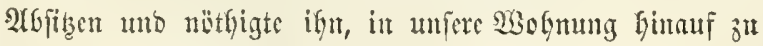

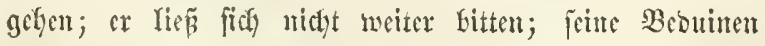
folgten ifm. Snocut or in miferen Saal trat, zog er feine Sticfel aus unb kefitieg ben Diwan, auf weldyen er

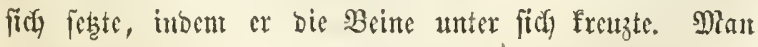

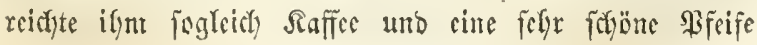




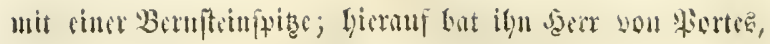

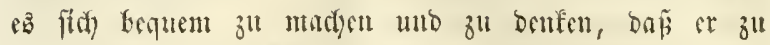

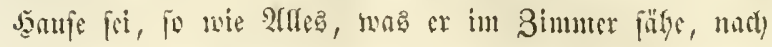

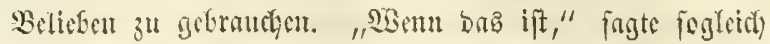

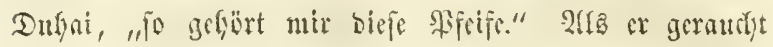

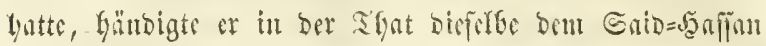

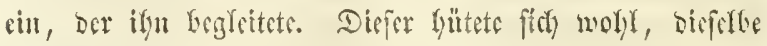

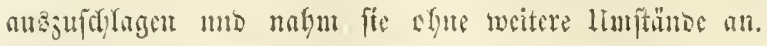

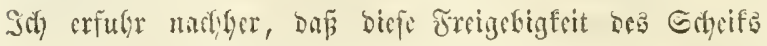
eir foron iun Loraus abgemadytes lleforcinfontuten mar,

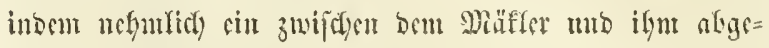
furlofiener Bertrag Dem Erfteren, it Der Cigenffyaft als Miafler, alle Gefdenfe you wenig Merth, bic ber Smeite

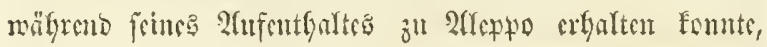
zutiduerti.

Serr wou Fortes hatte in sem anfópendon Simmer

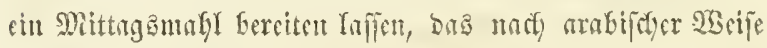

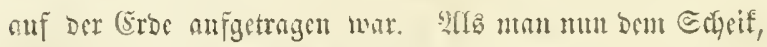

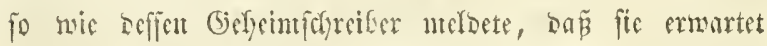

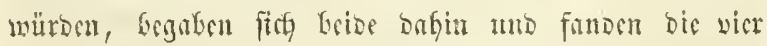

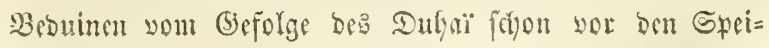
fen niebergefunct, wowon bereits sin grop̈er Theil unter

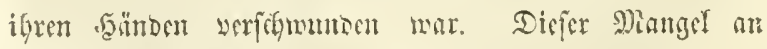

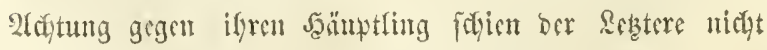
im geringften $\ddot{u}$ ed aufzurelymen, Denu or nafun obue

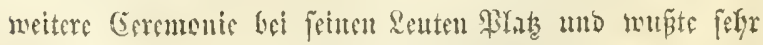
mobl die werlorente 3 cit cinzubringen. Da sic straber im 


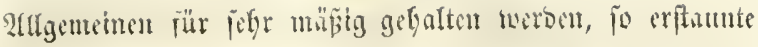
(ii) nicht wentig, sen Erfyeif mit femen senten bie

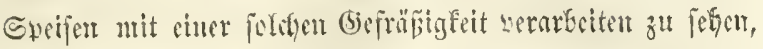

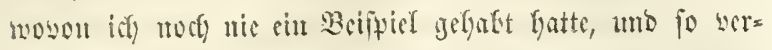

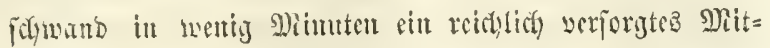

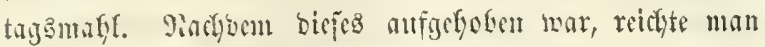

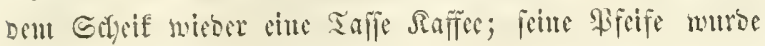
baju vou ciutem Bebumen geftopft mo angezintubet, bor fie ifyn fo yorbercitet barreichte; bie Interbaltumg besou

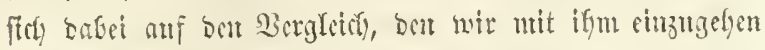

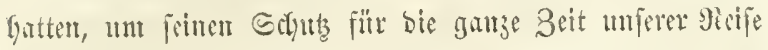

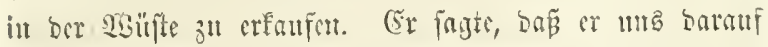
nidyt ummitteffar antmorten föme, baj or bie sifte ber

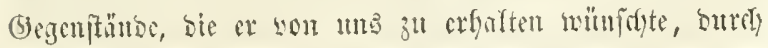

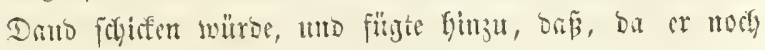

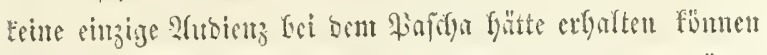
und erft ben fonmemben Tag angenonmen serben witrse, or batier verifbube, unb erit mach biefer Cermonic trie=

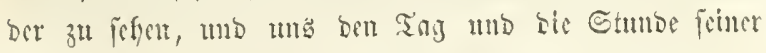
2efreife, fomie Den Sort, wo wir wieder jufanmentreffen

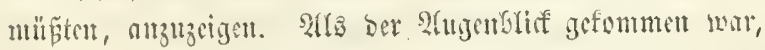
wo wir unz tremnten, grinjte unz Duhai, inben er ferite

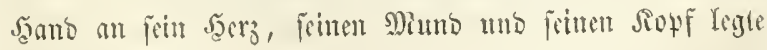

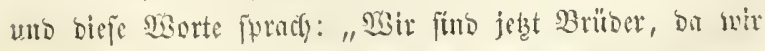
Brot und Garz zufaumen gezefien baten, Sgr fümt alpo

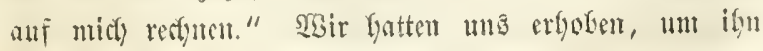

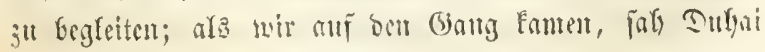




\section{9}

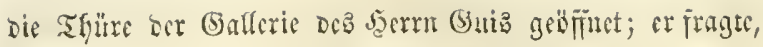
ob ex fith zu baup bifämbe, wornuf man ifym antmortcte, Daj er abmefono wäre. Dicfes ftörte ifn ater uidyt, er trat ein unt Durdylief bie ganje (5aflerie mit vieler 9ient=

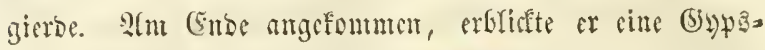

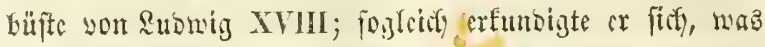

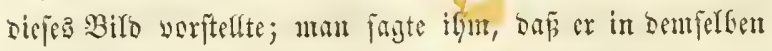

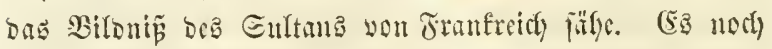
näber zu“ Ectrad)ten, entfobigte er fid son newem feiner Etiefer, ftieg anf cincu Diwan, welcher fith am Tüe beś

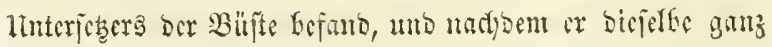

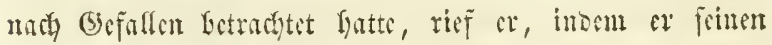
Finger an Dą Beficlyt Deß frligen Ribing legte: "Du bift fefr groé, aber Bott ift Dein Serr, ub Du wirft

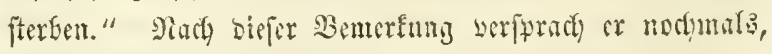
uns micber z" befuchen, for auf tho ritt uach fituent Rager juritu, bas or in geringer Entfarmung you ber

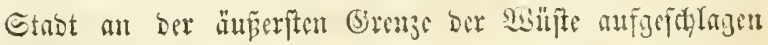
batte.

Den folgendor Tag bradite Dand bem Serm won

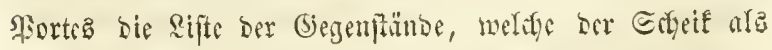
ben \$reis für feinen Sofuts werlangte; fie beftumben unter

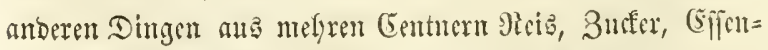
jen für bie Fraten uno zwei mo zmanjig yollftünbigen

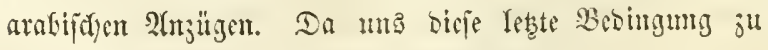

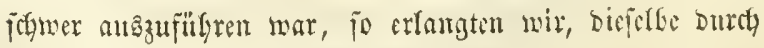

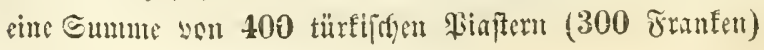




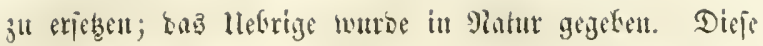
tebereutumft mar burdfan nothmentis, weil ab umb

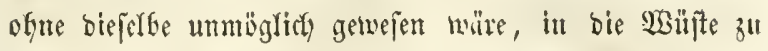
oringen; ca if ein Tribut, ben alle Europäer, bie fith

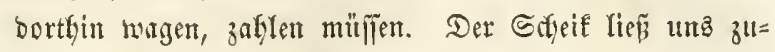

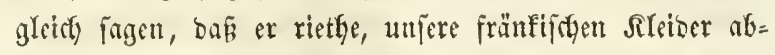
3ullegen und bie Sileiber ber $\mathfrak{B e b}$ tinenvölfer, bie unz

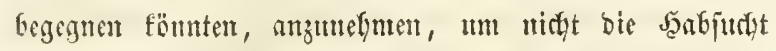
berifleten ju erregen.

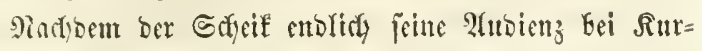

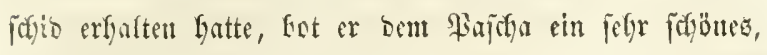

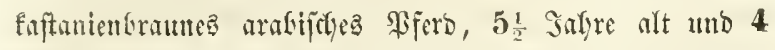

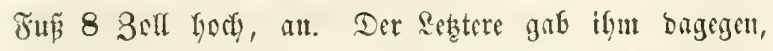

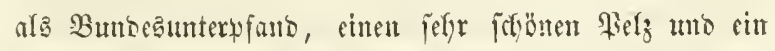

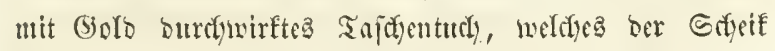
in Form eimer Şauptbinde auf jeturm Sopre antractete. Den folgemben Tag marfte er unz jeinen Bejucty in biejer

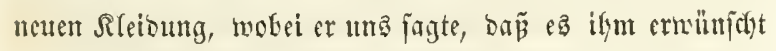
fein nüroe, weml tor in menting Tagen abreiften. Er

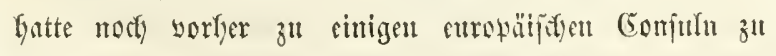

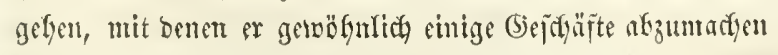

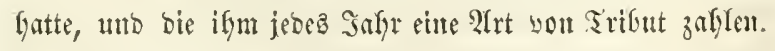
TBir werwendeten einen Theil ber Beit, weldye uns in

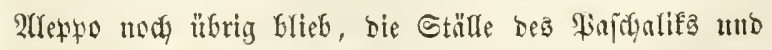

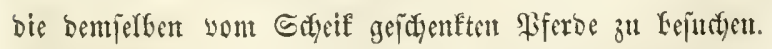
Bon allen benen, bie mir jafen, war bas Piferb, weldege

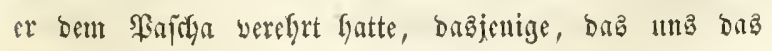




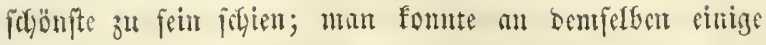

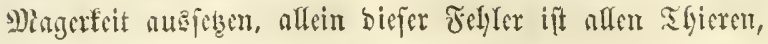
bie in ber Sighifte leber, eigent.

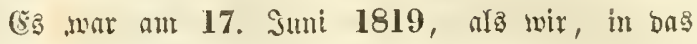
(5oftün ber Bebuinen gefleibet, endich afginger, um mit unferen Freutben in ber SפB̈fte jufanmenzutreffen.

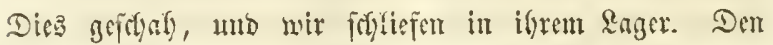

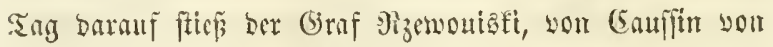

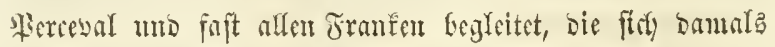

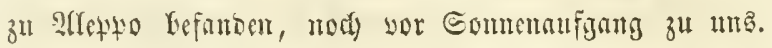
Diefe Jerren fauten, um uns cinte glürflidge Sieipe zu

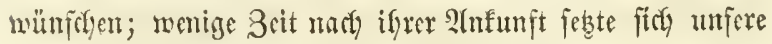
Saramane in Diaridy. Sie beptand an Den Erbif und fünfzig 2trabern, welche ifm biş zu biefem Theile ber Brenze Der Sibilite gefolgt maren. Dieje S(raber ritten

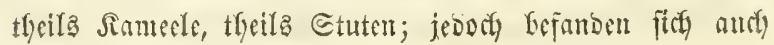
unter ifnen einige. Turfomamen, meldye mit Piferben be= ritten waren, unb bie auperbem noch (5jel bejajen, weldise

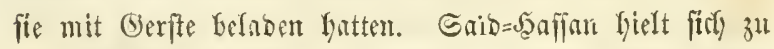
oen Resteren; jie nafmen ifn mit, bamit er ifnen, wie id foron gejagt habe, bei ibren sumecleinfünfen als Miäfer biente. Der Graf yon Jizenouizfi batte einige Diener bei fïty, unb bie Begleiturg bes Jecrm wou \$ortes

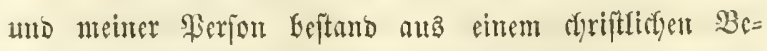

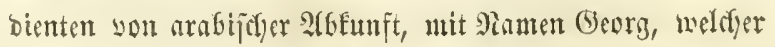
cint wentig Jrangöitic fprady; ferner in einem jumgen

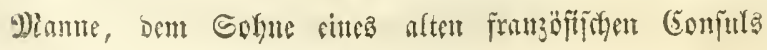




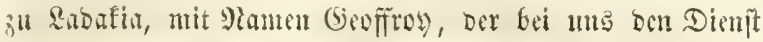

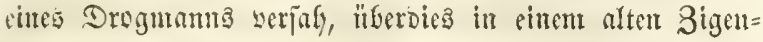
ner, weldyer beftimmt war, uns im Falle ber Poth als Eilfote zu sienen. 2Héperbent murben mir andi) von cinem armenijefyen Bcoientem, Mirgrebidy genumt, Eegleitet,

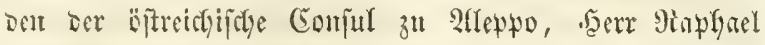
Witioto, mit bem Grafen jendote, nut einige Joflent cin= zแf

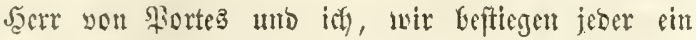

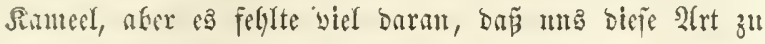

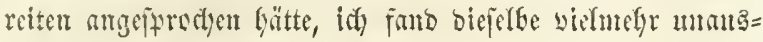
fleglidi, ba bab Thgier, weldes mir zu Theil getworben

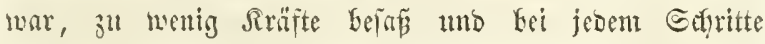

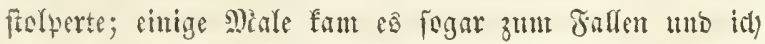

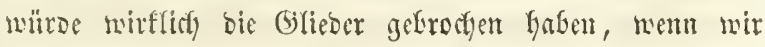
niçt auf cintem feinen und tiefen Eambe marjofitt märem,

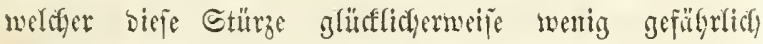

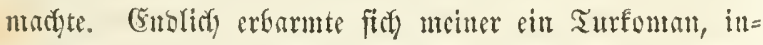
beut er mir fein Pifero anbot, bas idf mit bent berolidy=

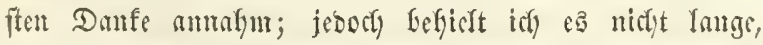

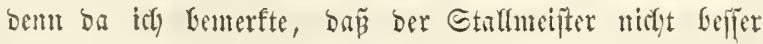
Darme max, als ich frither, fo beeilte ich mith, ifm mein

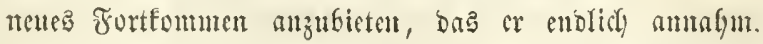
Sol) Eeftieg mun fein Drontebar, weldyes ficferer war,

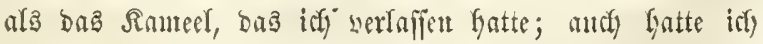

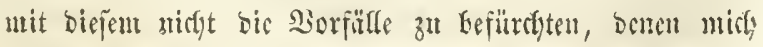
bos erfitere fo oft ausigeject hatte. 


\section{4}

Bier Etumber morftem mir etwa mariffirt feut, als ber Srycif bie Rarawatte bei einem fleinem Dorré,

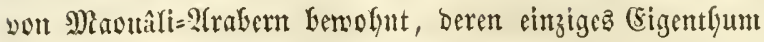
aus eintigen Bürifele = unb Edyafflecrben befteft, anthalten

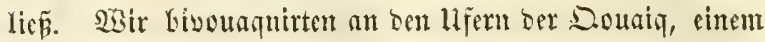

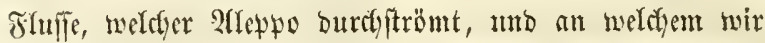
bie Butunen ciner alten Miüble jahen. Sein SBrifer war

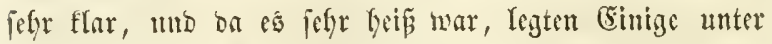

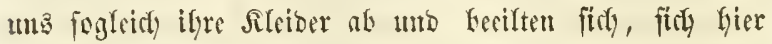

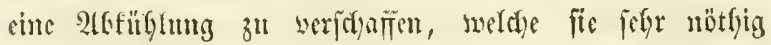
hatten. Das Bab verlajiento, gingen twir is cint, nach

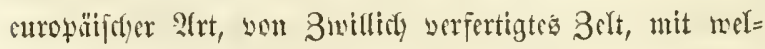
dyem wir แแล aแล Borfictyt veriefen Gatten, und in wel= flem wir umfer Magl aufgetrager fanden, ba wir uns

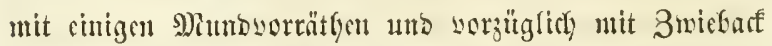
werjorgt hatter. Im 2higentliffe, als wir uns amforiften, ums üfer bas Miafy her zu mactyen, trat ber Sodyeif in

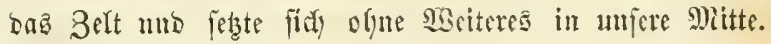

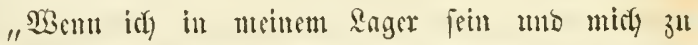
Sanje befüben werbe," fagte er za unz, "merbe id mir

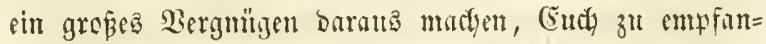

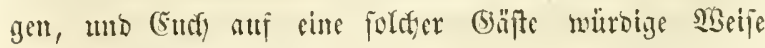

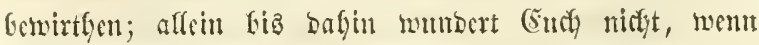

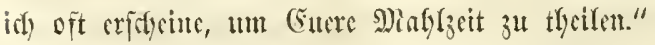

Dicfe Derfycipung war umöthig, un ums burch

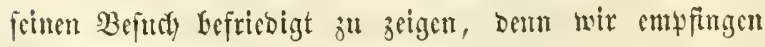

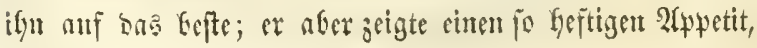




\section{5}

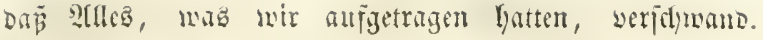
Den folgenton \$lorgen loben wit bas \&ager auf umb

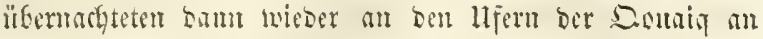
fincm Drts, welder unter bem Siamen Mierbj=cl=Sultani Eefamnt ift, mb ocr fidt auf ber Gtelle befundet, welche

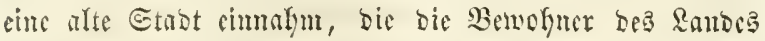

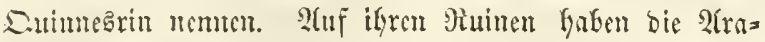

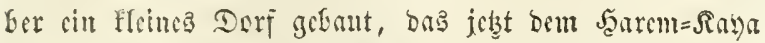

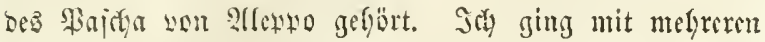
Basumen oabiu jpazieren mo mar fyer fo glütlict, cinen fleineu Borrath) won (Eim 3u finsen, ben id) fante.

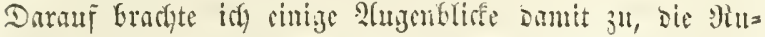
inen zu beputfyen; gegen uncin (Ermarten fand idf) fier

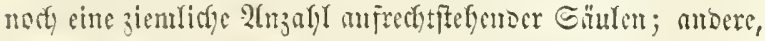

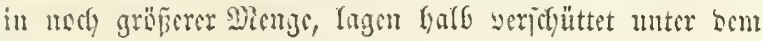
Gande.

Don folgemben Tag fotstem mir unjere Reije bben jo zeitig fort, ale bou verflofifench. Siarbont wir vier Etmoon lang auf eincm bremtemben Gambe marjefirt naren, crefisten wir endidy in Gïben eitren fleinen

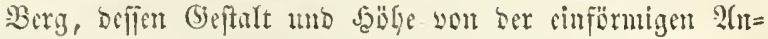
fidft Der Sandfläden, bic wir bis jest burdfftridyen hatten, angenefm abftact); Dericlbe mar ber Tel=d = Sultani (J̧il=

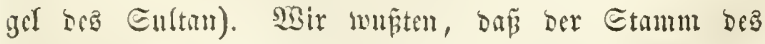
Dufgai, ben wir erreiden mollten, Den Jü bicjes fleiten Berges gemählt fatte, un jeime Belte bajel6jt anjujd gell; mau benfe jida balyer unjere llugcould, balo sort 


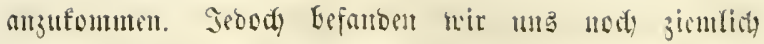
meit bavon, als fith plishlidy cinige arafifote Meter am

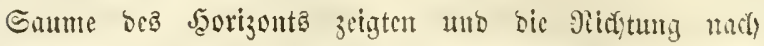
unferer Seite zu mefnen irfienen; pogleidy gingen einize Traber unjerer Sarnwane ab, bie Nenufommenten ju

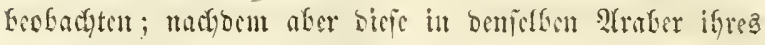

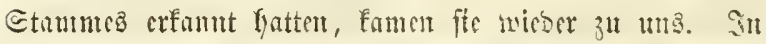

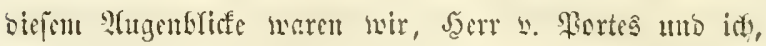
nur bejäaftigt, bie Joruten einiger you sen Siferben ser

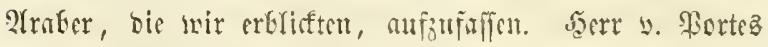

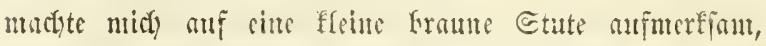

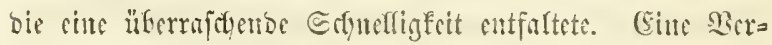

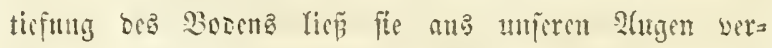

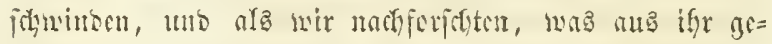
worsan fein fönte, forgen wir biefelfe in 3 orlanf cinger

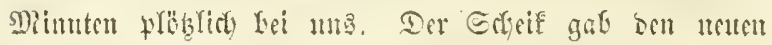

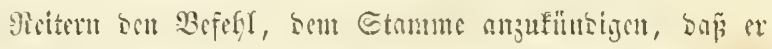
fíc! mit Sitrichio bercünset fyätte uno fogleich bas sager,

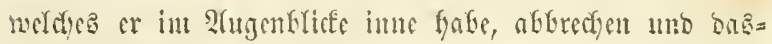

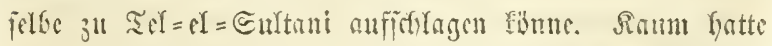
at bas ausgefyrodim, als bie strater ifye Piferse an=

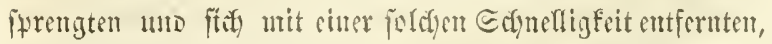

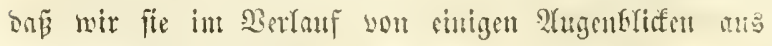
Sent Gefictst betloren Gatten.

Dicjer llmpano Gielt unjern Miarich niclyt iur min= boften auf; wir febten ifn fort, Insent nit un immer

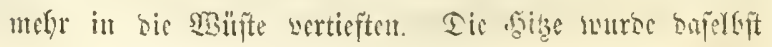




\section{7}

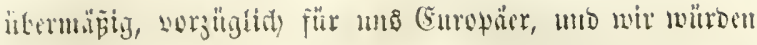

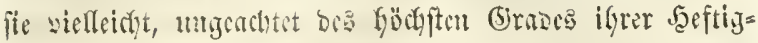

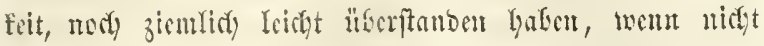

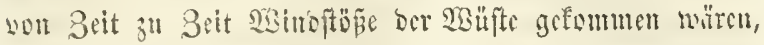

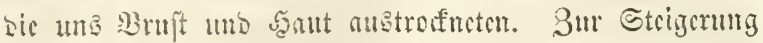

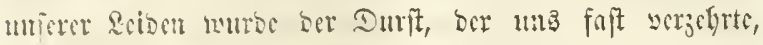

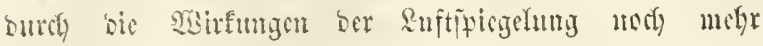

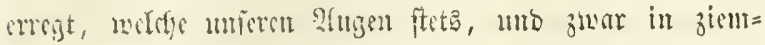

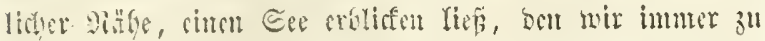

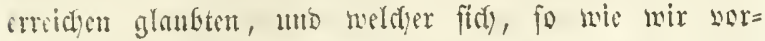

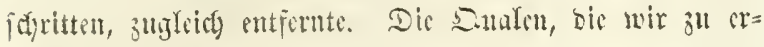

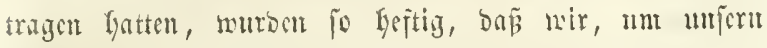
Eithurer ju mïpigen, ein menig yon ber Soume ver=

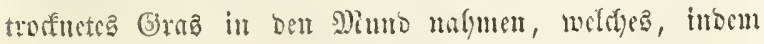

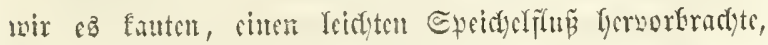

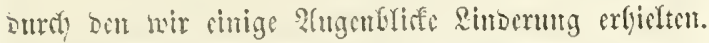

Nadjoem mir lange gonug maridfirt march, finten

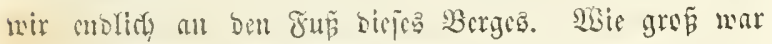

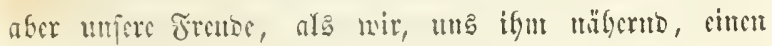

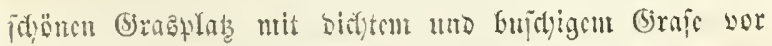

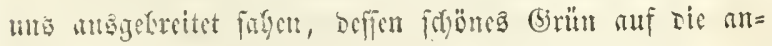

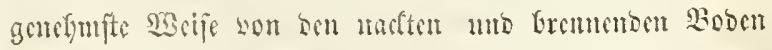

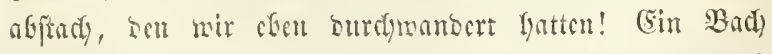

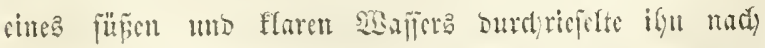

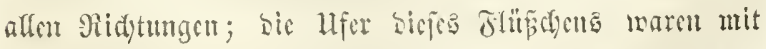

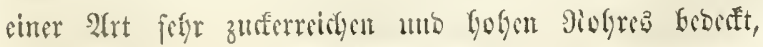

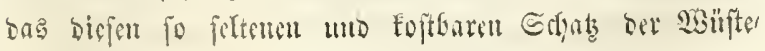




\section{8}

sen Blifen ibrer Bewolyer entzieft. Shie foubertar!

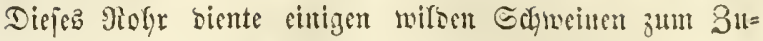
ffudftsort, bie wir, ohne cines einjigen habhaft meroen ju

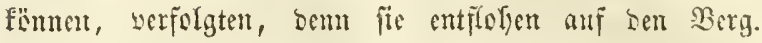

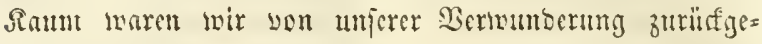
fonmen, ars ftef) Dubaï näberte uno uns Den Srt ans wies, wo wir unere Bclte attifislngen folltet. Die Tur= Fomanen lagerten umgerïbr fiefen oser adyt fumbert Scyritte עon ums jur Rectyten; Der Gryeif Yief baz jeinige in ber

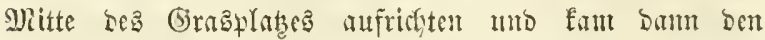
2tbent zu uns, um den Seft unjerer Sorrittye aufzefren zuli helfent.

In Den Miorgentumben bes folgenten Tages falyen

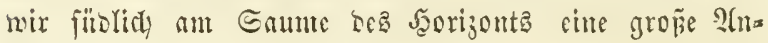
zafyl Samtele, bie wir für bie Jeerde von bem Stamme Des Dubaï anjagen; fie ublgerten fith einte febr reidblyal=

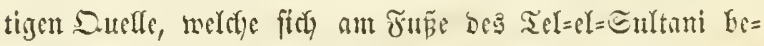
finbet, unb bie bem Bacte Siafrumg gift, bem mix bie freine Grasinfel zu bunfent Gatten, auf ineleber mir uns befanden. Dort angefonmen, wurben bieje Sfiere als

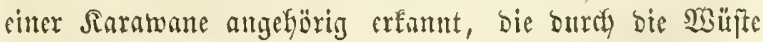
3og. Dufyai begab fith fogleidy zul ifnen unb forberte ben Tribut, ben alle Thiere, fomie alle Reijentoe, weldyer 2lugafyl fie audy feien, bem Echeif, befien (5ebiat fie burdu= wantoext, abtragen milffert.

Die 2lraber biejer fiemoen Sirmuane getjörten zu cimem Etanme, weldyer gemblgnlid in Eem Torile ser 


\section{9}

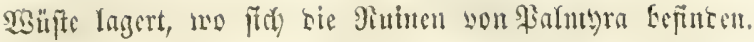
llnter Sem Nomen Gafunéc befunnt, betrcibt biefer

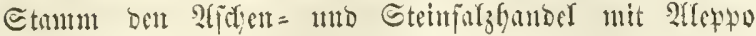
unto Solep. Die Sabl ber Santeele, weldye bicjen Sug aumurbten, founte fich auf orei lunbert Gelnufen; fie waren alle mit bicjen Gcioen Sanfmanangätern Eilalet

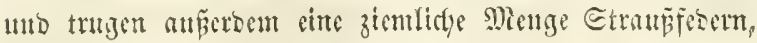
boren cinige Sarr y. Rorteß fuufte. Den andern Morgen begaben fte fid form 2rufgang ber Somme auf ben

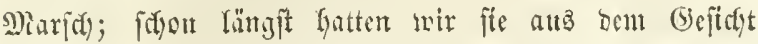
verloren, als wir segen zelyn Ulyr im Süben now sine

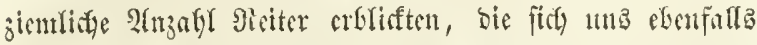
näberten; e⿱ war der \$ortrub ber Focoanz, beren Ectyeif Dutgai mar. Sie recognoseirten ben \$laz, wo fie ifyr Rager auffdrlagen follten; alle maren mit Stuten beritten, beren entige won ifren Fïllen begleitet ururben. Sic

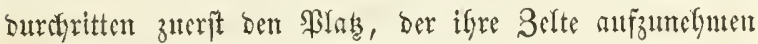

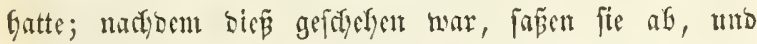

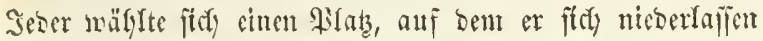
mollte, ffianzte jeine Sanze ars Beityen oer Befibuabute

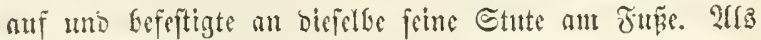
biefe exfte Jantolung abgetban war, begaben fich Talle in

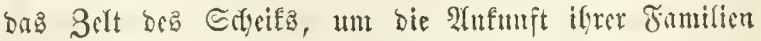
abzumarten. Sisir glansten fojon, Dafi biefe nifjt elyer,

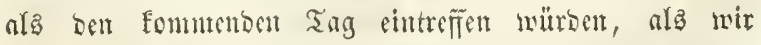
gegen 2l6ent ant fïblidyen Enbe bes Sorizonts eine grope Stanbwolfe auffeigen faben, und fo mie fie ficts 
uns nady und nady näferte, erbliften wir sine grope Menge Gelabener Rameele, sou ciner guten $2 \mathfrak{n}$ jafil $\mathfrak{A}$ raber ¿ébes 20lters Fegleitet. Somie die Saramane ins Rager

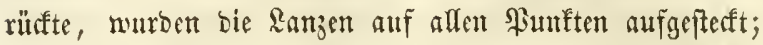

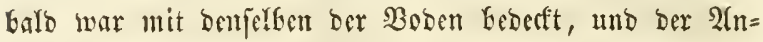

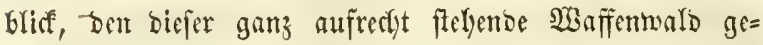
mäGrte, war nirflidy rectit furctttar. Der \$ilats, wo bie Ramje ftecfte, Fezeldynete bie Thüre jeber Beltea; Gald marem bie Beltpilörfe eingejaldagen, bie Reimwand mutroe aufgefpant, und jebe Fantilie fonute Befits won ifrer neuen Sisolyung nefmen.

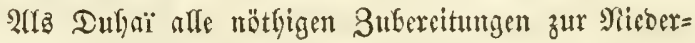

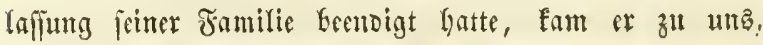

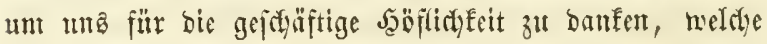

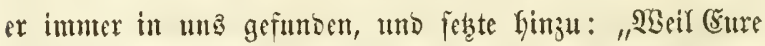

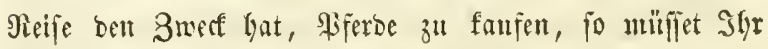
and nothwentigerweipe mit Gelo vexpefen fein, benn baß

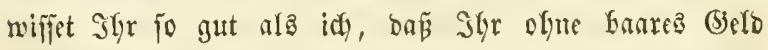
won umeren 2(rabern nicht cinen Ejêt erbalten fömt. Wenn ifh (Euch) aljo cinen Rath geben bari, jo weritecfet

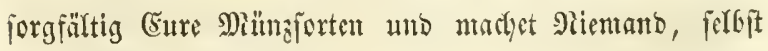
midy nidft, zu Euturm 3 ertrauten."

Diefe Difentyerzigfeit gefiel uma, wir bentb̧ten fie uno gruben mod Dent melyntlicken 2(bento in unjerem Belte

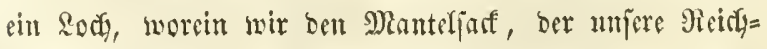
thümer einjoyloź, verbargen; Darüber breiteten wir bie Toppictye, welche unz anfatt $\mathfrak{B}$ ett bienten, aus. Später, 


\section{1}

als wir nötfig hatten, j" tuferem Geloe umpere 3ufludt

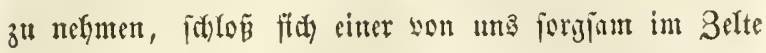

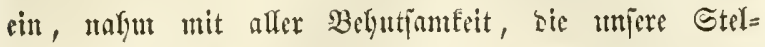
lung uns anjumenden getoot, bie nötgige Summe aus bem Manterjaf, mtd legte ifgn sarauf mieber mit ber gröpten Eorgjalt in Den von ums gefertigten

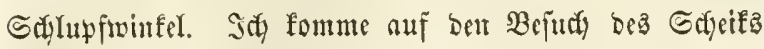

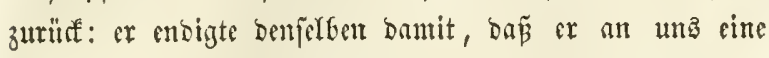

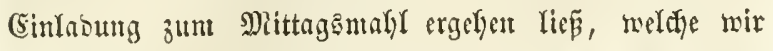
amtafyment uto ifm folgten. 2Tn ber Thüre fanton nir

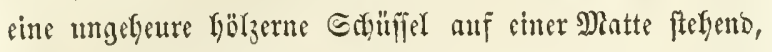
weldye mit halbgefodjten Sicis angejüllt war, befien

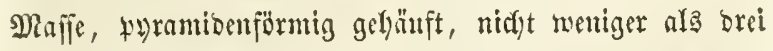

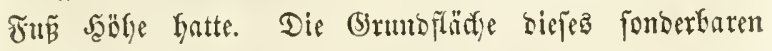
Gebüubç max, wie man fict) wafl denfen Eann, fefyr

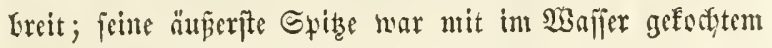
Saureelf(eifict)e Gelegt. Nadjoem uns ber Sedjeif bas 3ei=

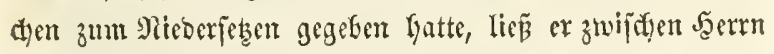

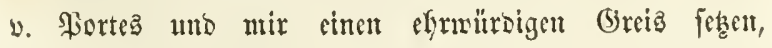

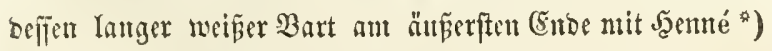
roth gefürbs wax.

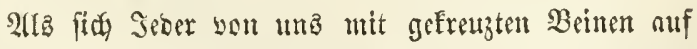

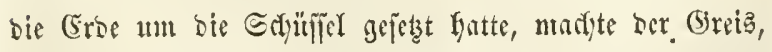
Den unz Duthaï aโs Nadybar zutgetfeilt Gatte, mit feiner

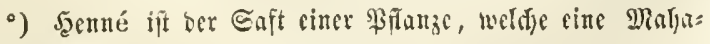
gะny= Farte gikt. 


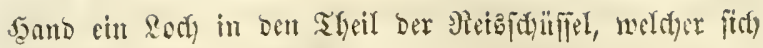
gerabe yor Serm v. Fortes und mir befind gof in bie Geiben Soblfungen Refen (geromente Mritst) unto nabut,

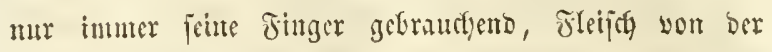

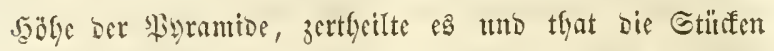
in bie grildy, sie er eben eingegojīen hatte. Coin Jeber that balfelbe, und nir waren nitfet menig in Berlegentheit,

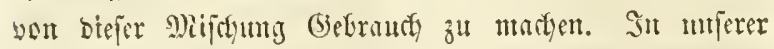
Dermirnung fafen wir auf unjere Rartharm und mafment

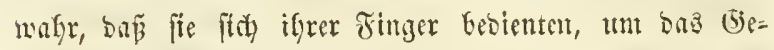
mifia), welifes Seber wor fith Gatte, in ben Mumb zu brin= gen. Sisir afmenten (b)ten nar) and bereiteten uns, fo gut

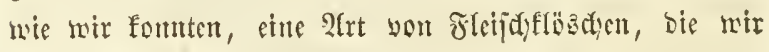

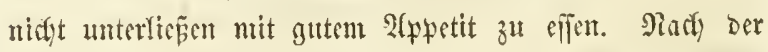
Mablzeit, die nidyt lange bauerte, enwarteten wir, Iaß́ man แแะ zunt 2(ufitefyen cin Zcidyen geben müroe; Dex

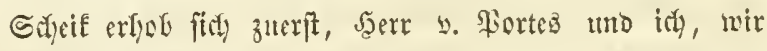
thaten baffelte. 2tnocre biffte, woldye voun Strfange ber Mablyeit an binter unz geftanden batten, warteten mur anf einen unbefetzten Slab, um pogleidy cintreten und fids

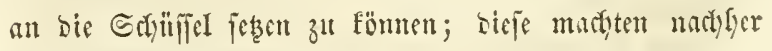

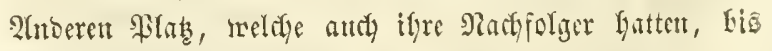

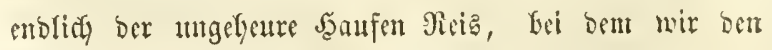
2trfantg gentadt Gatten, völtig verifomumoen war. So

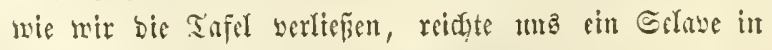

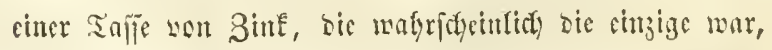
meldye Dubai Gejañ, oa jie allen Ditgäjten bicute, zu 
triufen. Der Extyif Ytés unz bann in fein Belt get)en,

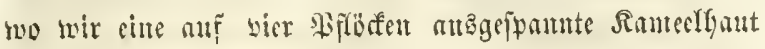

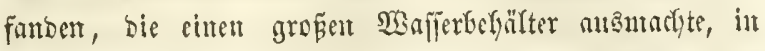

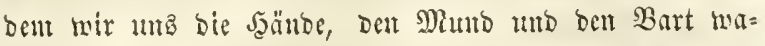

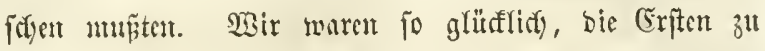

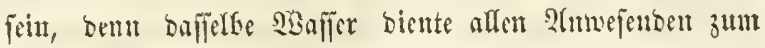

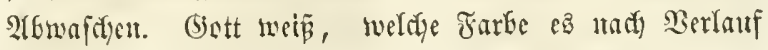

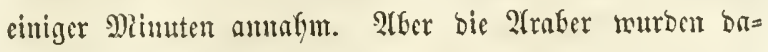

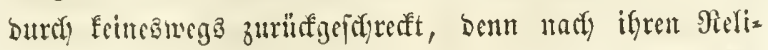

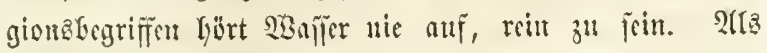
wir bicje unvermeislidye Ceremonie wollentod Katten, nafhu

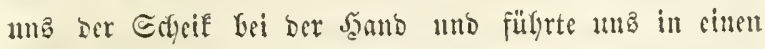

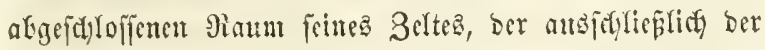

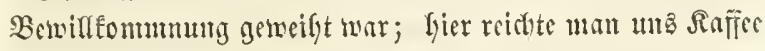
in cimer Tafie, weldye, wie bie won 3 inf, beren idy ofen errnälyute, cbenfalls you cinem (bajte zum anbern manberte. Der Raffece murbe ofye Butfer getrunten, und ala Seber

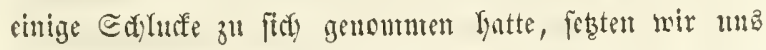

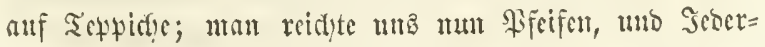

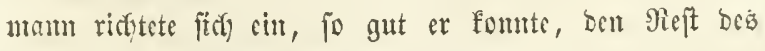
Tageb ofye Rangemeile zuzubringen. Die jungen Rente untergieften fid mit Singan, indent fite fitch mit cinem Snftrmunent Eegleitcten, weldfes aus eincm zugefchnittenen

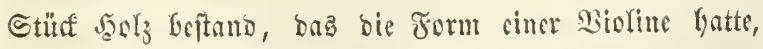
und anf melcyem cin Ctïf gegerftes Ramedfoll befejtigt

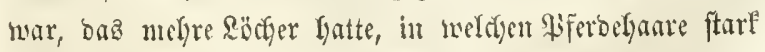
eingejpannt maren, bie ben Dienft ber Saiten vertraten. 


\section{4}

Der Siolinbogen, befien fie fich bebienten, beftand aud aนล \$ferbelyaren; bie Töne, weldye er Yerborbradyte, wa

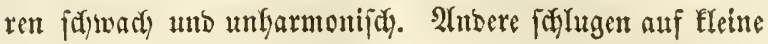
metallne Trommeln.

Sn einem autoern Theile bes Beltez, im Mittelpunfte

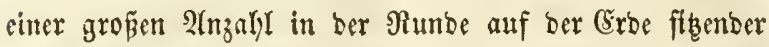
Bebuinen, ftand einer jener Errzügfer, bie man im Drient aui allen effientlictyen Dertern uno allen $\mathfrak{A}$ tulyaltefunften ber Sarawanten finbet, bie ilyr ganzes Reben bantit zubrin= gen, Mäbrdfen zu erzăglen, weldye benen bei uns, unter bent Pamen: Taufetto und eine Nactit, weber an Ränge, nod) an frudtetbarer Eintildurgafraft nadyftelyen. Daz

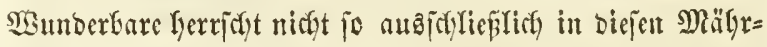

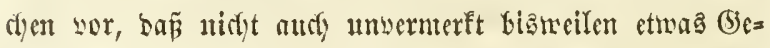

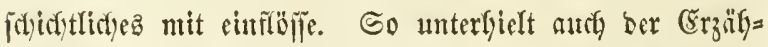

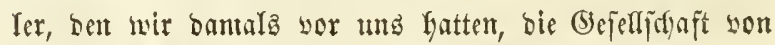

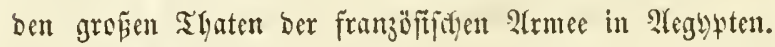

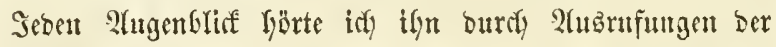
Jurclyt, bes Bergnïgens ober ber Bemutuderung unterbredjen, beren Rärm mir faft bie Dhrent zerrif; bafei befanto idy ntidy swie auf ber Folter, ba idy bie $\mathfrak{l t r}=$

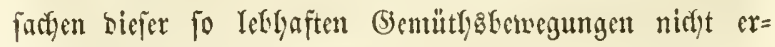
rathen founte, als Secr (Beofirroy, ber uns als Drogmam biente, uns entolide barïber belefrte atto fick erbot, biejen

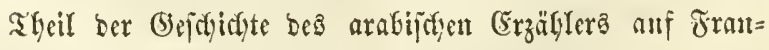

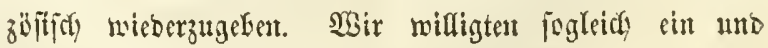
erfulyren Folgentoes: 


\section{5}

Die Framzojen, jagte ber 2 (raber, find übernatürlicte

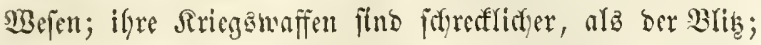

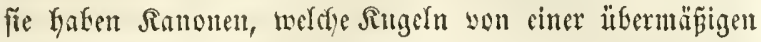
Grö̌̃e in bas Rager iffer Feinbe fallentern; o wie jon= Derbar! - Defters bleiben bieje Sugeln einen Shugenbliaf unberveglidy liegen, nadtber, wemn man am ivenigiten

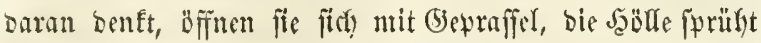

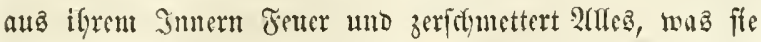
ungibt (bie SBomten). Noch metyr, fügte or fyimu, fite

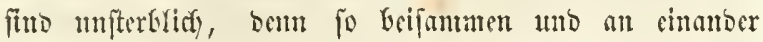
gefettet fie anded marichiren, mag man alf fie forjeçen

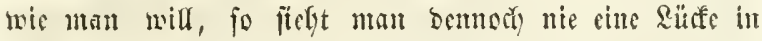
iffren Peiben. Sie Gaben überbiés bie (bemalt, fid) nady SBiflen ju vervicffültigen, benn oft fitht man eine fleine

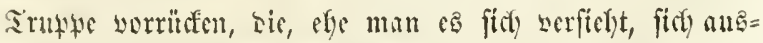

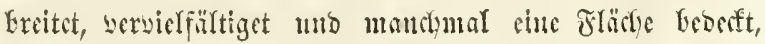
moyon fie worber mur cinen fleinen siunft eimnalymen

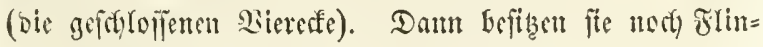
ten, mit benen fie oít fünfzerlu ober żwanzig Mial fortic=

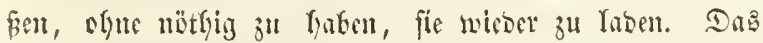
ift cin nie aufförembes Fener (Rinient= 1mb Pelotonfeuer). Esa gibt unter ifuen Eoloaten, welche große baarmitisen tragen; ofy! biefe erft fitto furctetbat, bonn cin cinziger ift

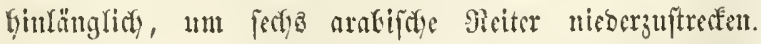
Shre Pieiter Yingegen finto nicyt zu füroften; cin eingiger

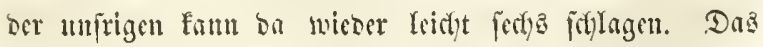

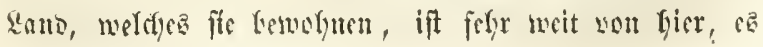




\section{6}

ift Durif) Laz Sleer (Baar) yon unz gefdieben. Nun!

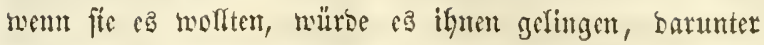

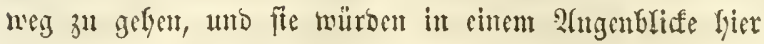
anfoumetr.

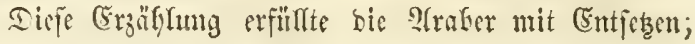

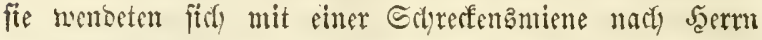
v. Fortes und mir, als fucteten fie in unjeren Blicen

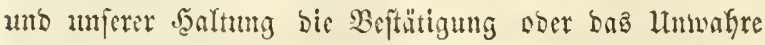
ber munberturen Dinge, bie fite fo cfen gefört hatten.

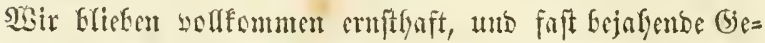

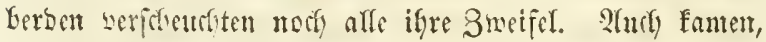

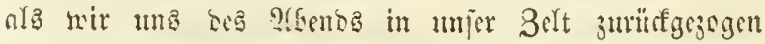

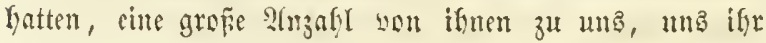
Erffannest und iffe Bemunterung zu Kezeigent. Diefer

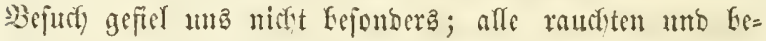
fïcten mfere Soptiote mit ibren süujen in unfägliefer

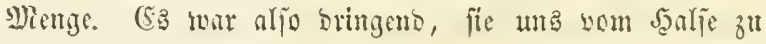

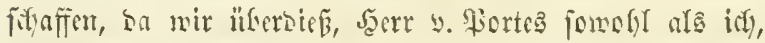

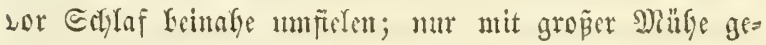
lang ç uns enolich, fic żn cutfermen.

Inoen ith ben folgenten Tag in alfen Theifen bes Iagers fpajieren ging, arfeitete idy midy surety ben zalyl= reidfen Eefilf, welder au bent Bacte mudte, ber burds

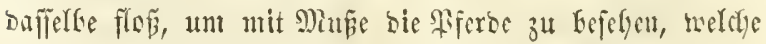
bierfer gelanfen fein fonnten. S)icine Inftrengungen nurbon belohnt, benn irf lante Gielegentyeit, eine Stute

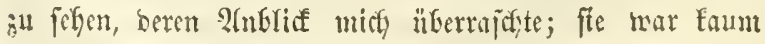




\section{7}

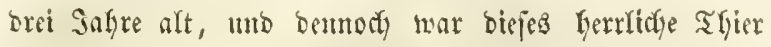

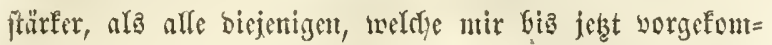
ment maren. Saty zeigte biefelte bem Brafen Mijemouiati,

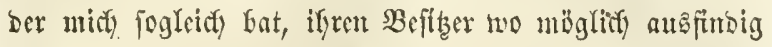

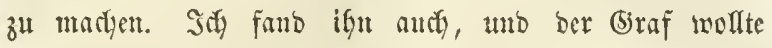

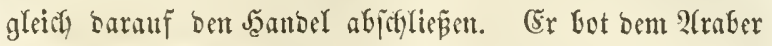
80 Bentel $(30,000$ Staufen), unb bicjer figien bamit

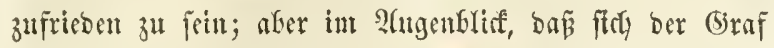

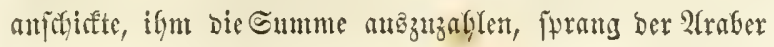

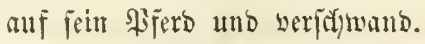

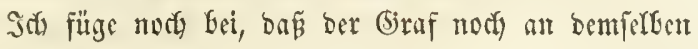

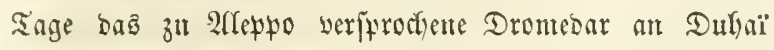

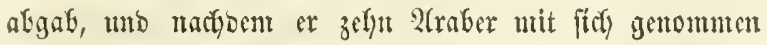
hatte, bie ifm als Bedeffung bienten, ging ar ben 2lfent ab, um bie nody immer fo fojönen und fo impojanten Siuinen you Falmyra z̆l beju(t)ent. 


\section{Fủnfte Inatel.}

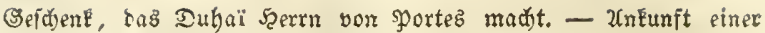

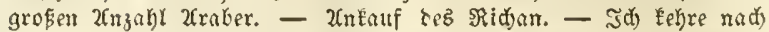

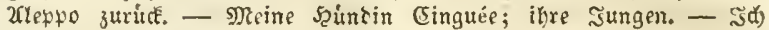

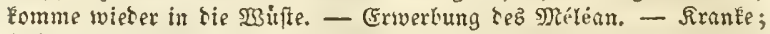

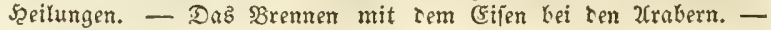

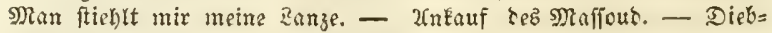
ftabl im turfomanifden Lager; nábere umftánte; bie geftoblenen

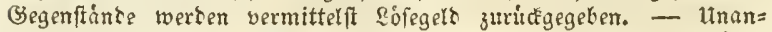

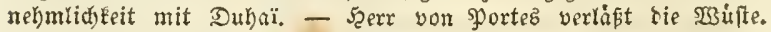

Šinige Tage nady umperer Ragerung zu Tel=el=@ul=

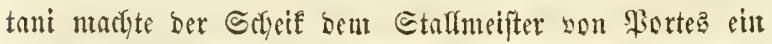
(B) biejes Thier Gatte eine gefällige Jorm mo ftaunte won ber Mare Roélan. Şir gaben ign den Nanten Dubaï.

Der Stamm, bei weldyem mir waren, war nicht Der cunzige, Der sent Dubaï gebordute; biefer Säuptling be= febligte unter benu Sitel eines Sdyeif $=\mathbb{e l}=$ Sebir noch brei

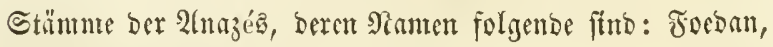
2lboaal und Sabaaf. 2rlle brei lagerten inmer in cini= ger Entfernung yon cinantor umb befapen bie berïbnteften

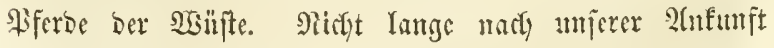
fidiffte bafyer Dubaï einen Sameeltreiber an jebent ber Gtïmme, um ifnen refannt zu marten, bafi Franfen mit

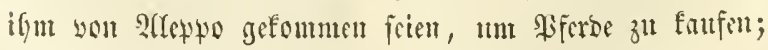

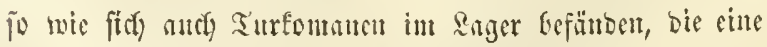

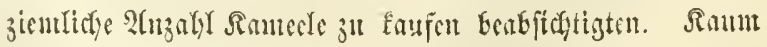
waren zmei ober brei Sage nach bem 2 Ggange bs soten 


\section{9}

verganyen, ala wix cine Mienge Mraber in bent Raget an= Eommen faben, bie uns jogleidi ifge jumgen Pferbe von zwei, orei und vier Safyren vorfingrten; uter biefen fan= ben wir mut eine febr fleme 2(nzafyl, welefe wärbig ge= wejen wäre, SBeiduäler abzugeben; anch Eauften wit nur

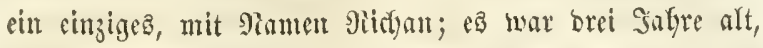
Gatte grautes Saar und war mit einer atbletifatyen Struftur

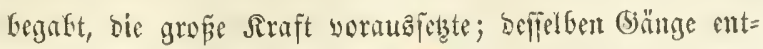
foradyen jeinem Rörperbane. Sinige Tage narber nötgigte Den Dubai ein Bote, nnwergüglid) nad 2repto abzureifen. Serr won \$ortes Geftimmte mids, ifgn zu Gegleiten und

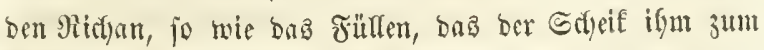

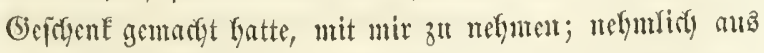

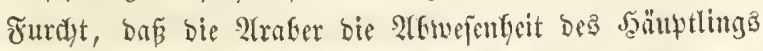
benuzen mödten, um ung bie jungen şbere zu fteblent. sor) Yieñ midy nidjt Yange bitten, unb ben andern Tag

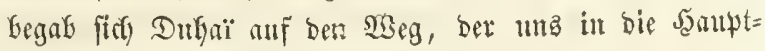
ftaot Des Fajualifis fügren follte.

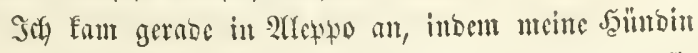

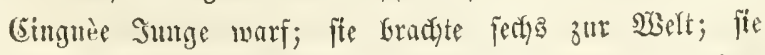
biejelben alfe exmäbren zu rafjen, wäre für fite eine $3 \mathrm{Z}=$ mutfung getwejen, weldye traurige Folgen bätte nady fith jiefren fümen; auf ber anderen Geite erfigienen mir biefe

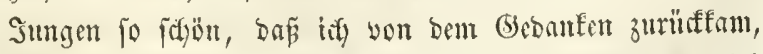
fie zut töbten. S(4) fomanfte aljp nody ungemié, als ith

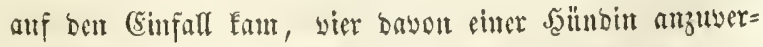
trantu, bie fith zufällig gerabe in bericthen Rage fefänoe, 


\section{0}

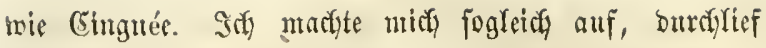
bie weriatiedenen $B a z a r z$ ber Stabt, fing aber fiton an

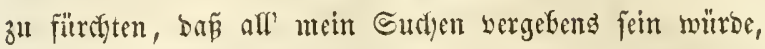

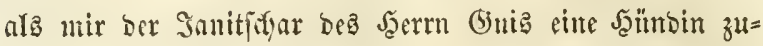

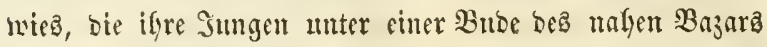
beim Eonjulat gemorfen Yatte. Sch fatte biejen müróis gen Minfefman mit meintem Sorlyaben befannt gemadyt, Dafer ging or zur felumbin, gab ify, um fie folgajam zu nactyen, ztt freffen, und ben ambern Tag getang

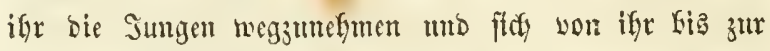
Shÿr unjeres હtallea gefolgt zul feften, wo er feine Bürbe

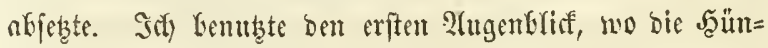
bin abmejend war, um iffr eines ber Sumgen ber Cinguèe unter bie ifyrigen ju fterfen, unb wiebertyolte befejes 2 Berfah $=$

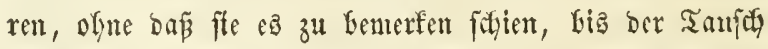
fertig war. Die meinigen murben yon biefer arment

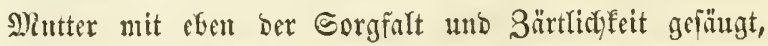

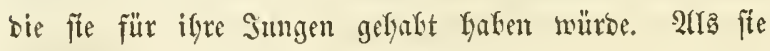
grof waren, gab ict) einen Serm (5)uis, ben amberen . Ilaron, ber im San molyte, und ben wierten bem fpani= fdifn (Eonjul; zwei Glieben mir, won benen idy einen

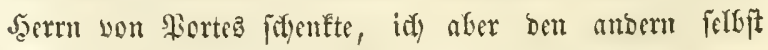
befielt. Der Etallmeifter befibt ben feinigen nods, ben

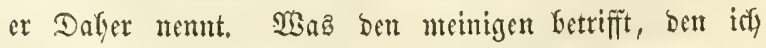

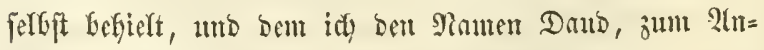

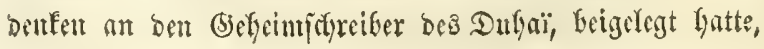




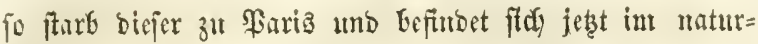

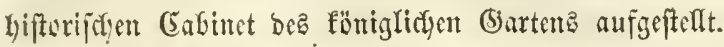

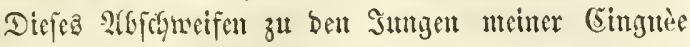

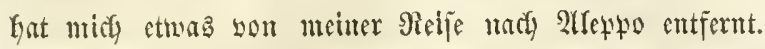

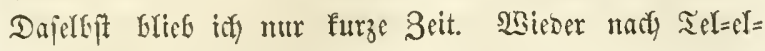

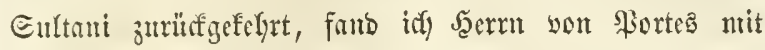
cinter neuen (Erwerfung bereidyert;

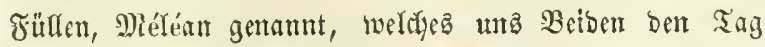
yor meiner 2(Greije burdy feine Form und Stärfe atī= gefillten max.

Sarm war idf angelangt, afs iad ju cinem armen Teufel won Turfomanen gerufen murbe, ben cin Giorpion

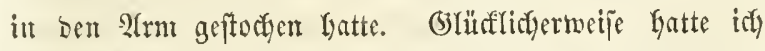
einige $2\left(r_{3}\right.$ meimittel bei mir, unter benen fidd auth cin

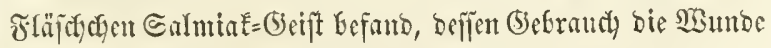
felyr balo Geilte, bie burch ben Stidy bes giftigen Snfettea Yyerbeigefïlyt worben mar. Dieje fodnefle Sur erregte bie

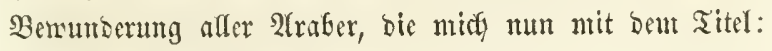

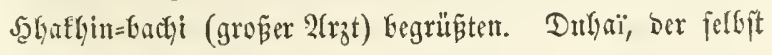
Dariliber erftaunt war, yatte fofon feit lamger Beit an

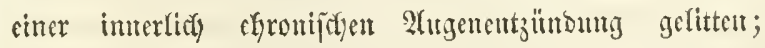
cr befragte midy bafer über bie Mrittel, tgun wicber Ger= zuffellen. Sath riethy iffne ein 3ugtflafter an; weldyes Mittel Fïr ifn eine unbefantue Gadye war; id erbot midy alio, ez ifym zu legen, waz er jufriesen mar. (She idf bas betwerffelligen fomte, riffrte idd ifgm bie Saare

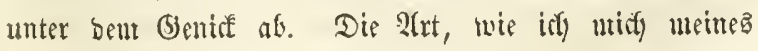




\section{2.}

Majirmeflers bebiente, bie Gefdntubigfeit und Metrigfeit

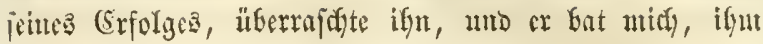

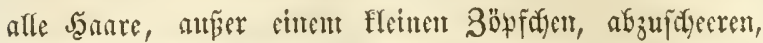

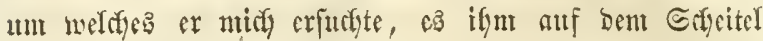

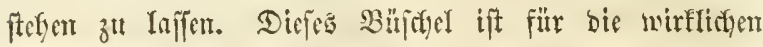

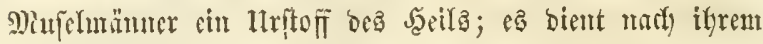
Tobe sem guten Engel zum Mrittel, fie fräftig gemug

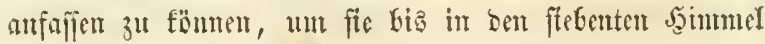
zu erficken. Nadyoem dicje vorläufige Dperation voll=

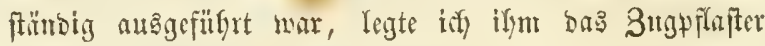

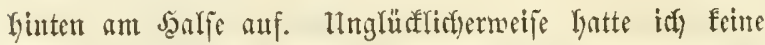
Binden sajpelbe zu bereftigen; in neener Berlegenlyeit ftand idf nitgt lange an, meinen Turban zut offerts, imbent

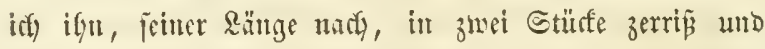
ifym won ber beften Sälfite cute Salabinde ntadjte. Narfy

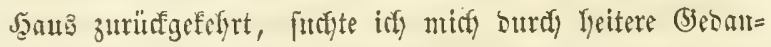

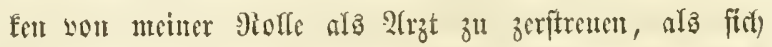
Dulyai, an ben icty nisfyt mefyt Dachte, plöblich in meinem Brlte zerigte; of fan mit blofent Salfe unto bat Sopr

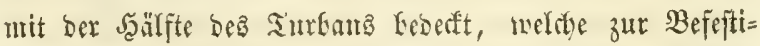
gutug Deß \$flafters gedint batte. S(a fragte ifu baber, warum er fein Bffafter fo rafo neggeworfen hätte.

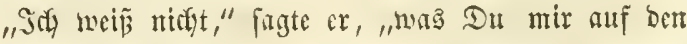

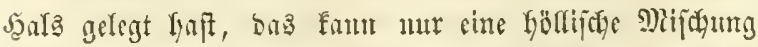
jein, benn nie gate id jo ftedyende Genuterjen erlitten;

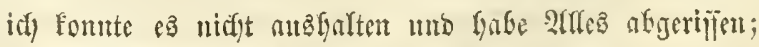
tunjento Mral will inf lieber meine Altgen werlieren, als 


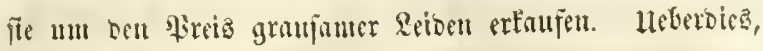

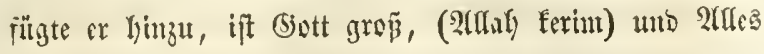
fefet gefictriefen."

Osgen biefen emigen Beweis̈grumb ber religiöpen

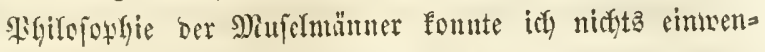
oen; idf) fomte ifn mur gutfeigen. S(t) wartete mux onrauf, wenn es ifm gefallet wiltroe, midy mit bem

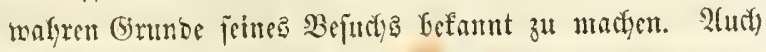

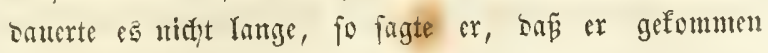
wäre, mich zu erjutdyen, thn zu einem feiner Sermansten z" begleiten, welfyer gefïfyrlidy franf fei. Sit) zëgerte nicht und befand midy balo an einem Belte, an befien Thüre ein armer Sterbenber Iag, weliter, auf bent Santbe

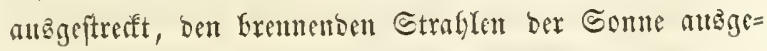
fest war; Derfelbe Gefand firif unter bent Sänden jweter 2(raber, weldfe unanfgörlicf) bemüht waren, feinen Sörper nit fein gepulvertent Samtedmift zu beftreuten, ber beptimmt war, ben Sifweín zu verzelyren, welcter won allen feinent Griedern Gerabifló.

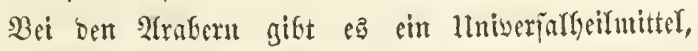
welcter bie (Enterifiation *) ift; bieję Mittel wiro ges gen alle Hebel, bei Allem, mas Refen bat, angetwendet; bei Menfden, jo wie bei shieren. Fitylt eist Straber cinige Solif, fotuell fringt man bas ซreuer auf ben luter= leib; hat ex Brufjictumerzen, jo bringt man es auf bie

-) Das Brenen mit cinen glüfentert Eifer. 
Sipwen; Dber hat er Ropfmel, fo wirb bas feuer auf

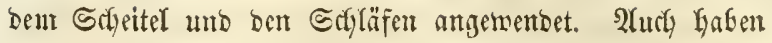
faft alle Bebuinen, junt ober alt, Ermadyfene ober Rintoer int erften 2(Yter, Den Leib mit Feuernarben gezeicifnet.

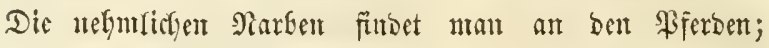
bem daz Brennen ift im allgemeinen (bebrauch, um alle fohwaden Theile biejer Thiere zu ftürfen. Die DGerarme,

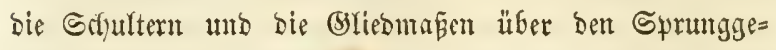
fenken finto bie Theile, wo biệe Marben gemeiniglida be=nerffGar find.

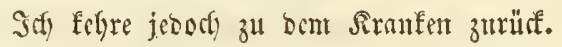

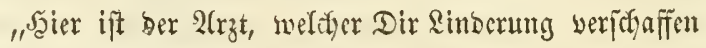
miro," fagte ifyn Dufaï, fict) ifgm mägerno.

Der 2 frme brefte feine fterbenden 2 lugen madh mir und jagte mit Mä̈he, indent er mir mit bem Finger ben

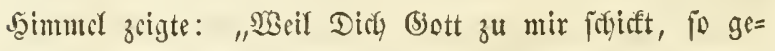
forieglt

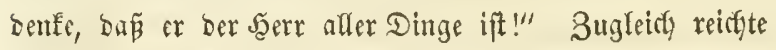
er mir feinen $\mathfrak{2}$ (rm, um rach bem $\mathfrak{P u l z}$ füblyen zu Yafïert.

S(t) war nicft mentig verlegen, benn fo frane, mie

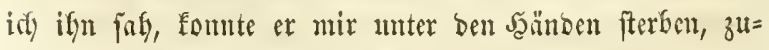

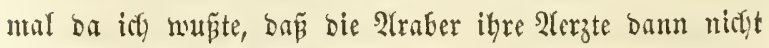

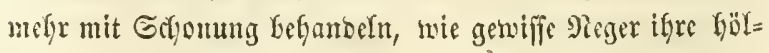

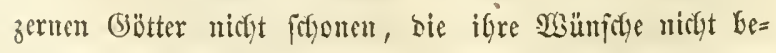
friebigen, uno fie werferemen, un bieje, ifrer Dhnmacht

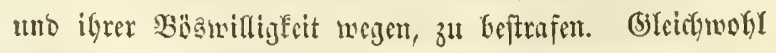

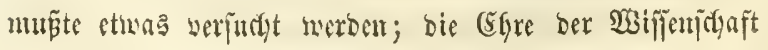




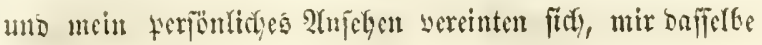

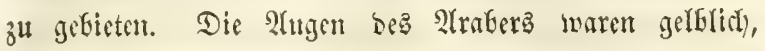
unb er batte öfteren Trieb zun (Erbrechen; diefe Szmptome

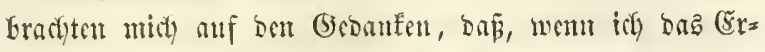
brecten erregte, idf ifm vielleicht eine Erfeidyternng wer. foraffon würos. Sch lię̧ bafer zmei Gran Emeticmm in zismlidy bünmen Peišmaffer auflojen, bas cr felyr verbümnt uno in Mienge nalju tho weldes auth Gald wirfte;

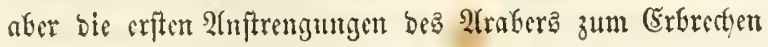
Eradoten (Entjeten mater Den Alnwejenden Gerwor. Sot)

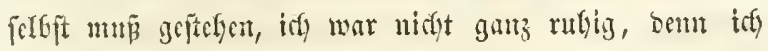

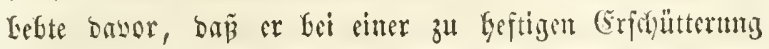

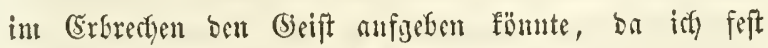

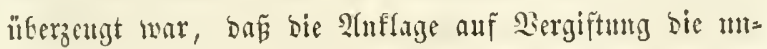
mittelfare Folge eincs folthen Borfang fein mürbe. Dods

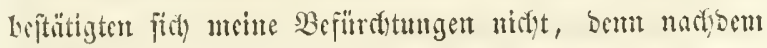
Ler 2traber cine grope Mienge Gulle meggefrocticu batte,

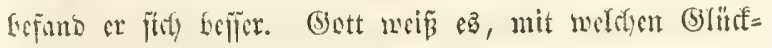

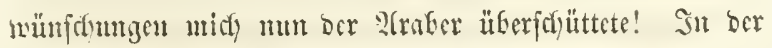
Srigiçung friner Danfbarfeit legte er mir fine Stute,

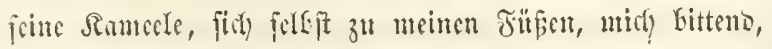

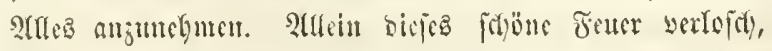
fo wie or an Srifftert ơnufm, und ars or wieber gebon fomte, io vergingen melyre Tage, ofne oaj ein Raut ber Danfuarfeit ïber feine Riften ging. Sor Gatte aber fordon

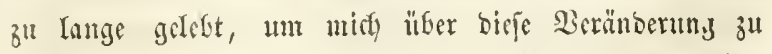

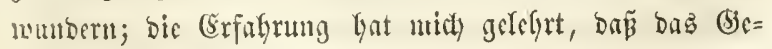




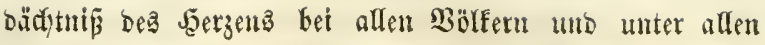

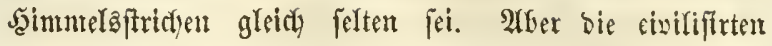
Böfere milien wentigftens bieje Bergeffenlyeit unter Sprady= formeln zut verbergen, bie für igren guten SBillen fpresten,

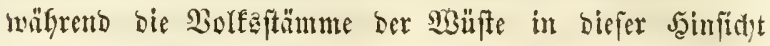

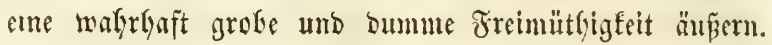

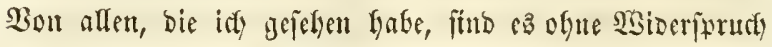

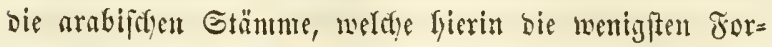
men beobadyten.

Şie bem andy jei, biefe baeifung bejeftigte body

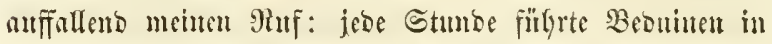

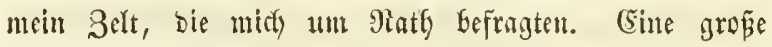

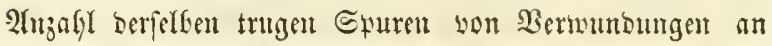

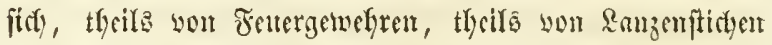

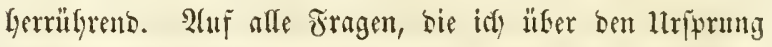
mb bie ltrjadje bicjer Siarben an fie ridgtete, mar nidyt

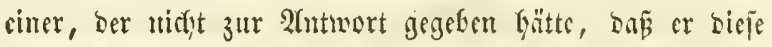
reim Plündern einer Saramane erfyaltem Gätte.

S(1) Darf nicht vergefien, eines felyr jonberbaren Dieb=

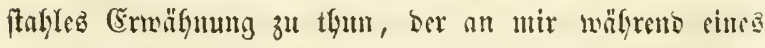

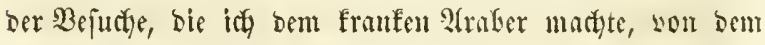

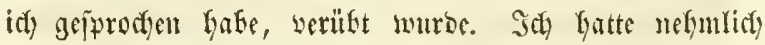
meiu Belt werlajeen, nadjoem (id) meine Ramze an ber

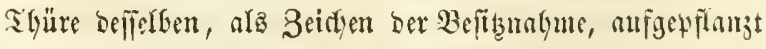

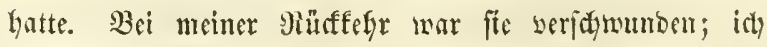
silte baher jogleidy zu Duffai, um mich) utber biejen Dieb= ftafl zu Feflagent. (Ex börte micy lacherto an utho fagte 
mir, bás id bartiter nidgt erftaunt fein follte, oa ich midy in ser Siltte eines Bebuinemitammes befü̈tbe; allein

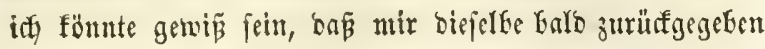

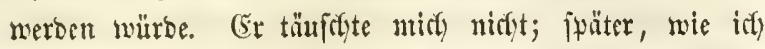
es ju feiner Beit mir zu erzätlen yorbelhalte, wurbe fie mir zurürfgebractyt.

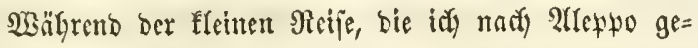
madyt hatte, war Şerr v. Fortes mefre Mate um eimen vierjäf̧rigen Brauner im Sambel gewejen, ber allemal

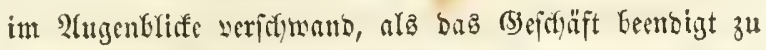

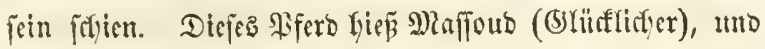

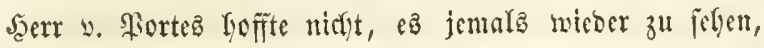

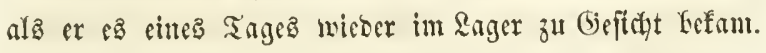

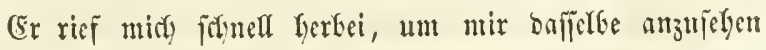
unb won nit äber baffelfre ureime Mleinumg zu bören.

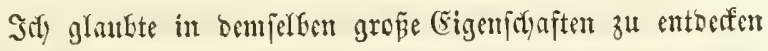

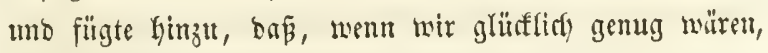

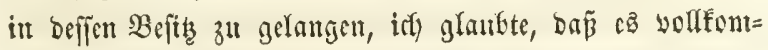
men für bie Miormanbie pafjen mürbe. Mafifonto ver=

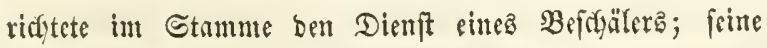

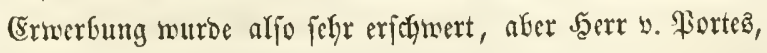

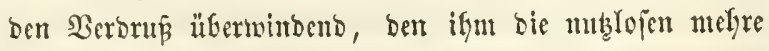
Mlale wieber angefnüpften unb inmer wieber afgetroche= nen Ilnterbandlungen verurjad)t hatten, wenbete fidi) an

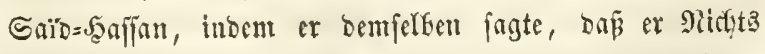
verfämmen mö́ste, um if̧m, mo mögliç, ben Beouinen,

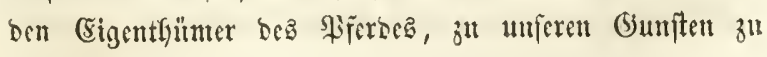




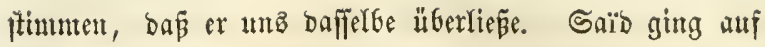

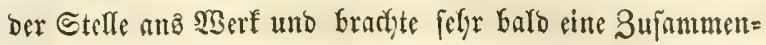
funtt ber Barteien zu Stande.

"Thue uir Dein (5ebot," fagte ber Beoutine zu Werrn

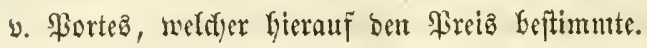

"Biete noch mefre," ermieberte ber Bebuine.

"Sinen 2lugenbliaf," jagte idy jogleicy zu Reterterem; "iri

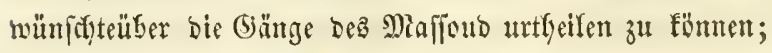
fömnteft Dut ifn nicyt ein paar Minuten reiten?"

"2્Re Bebuinen swiffen, wie er gefyt," antmortete mir fult fer 2(rater, "idi) weroe alio nidjt thum, was Du ver= rangft. Sebver)," füigte er nady einigen sugenbriafen bez Grillifymeigen Ginzu, "willit Du ifn ourdyaub unter bent Meiter felyen, fo reite ify jelbit."

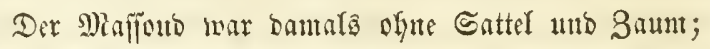
fetme erfatreffiche Mingerfeit lyatte ifm auperbent bie Sairbeliäule aufierorbentlict Gerworragend gemadit; firt) ifm alio in siefen Buptande anzuvertauen, erregte bock

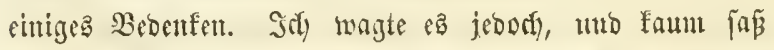

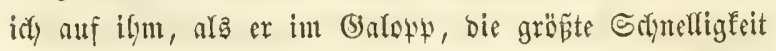
entfalteno, sawon furengte. Die eriduresficife Dianter,

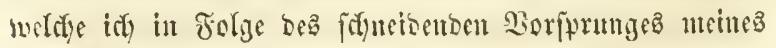

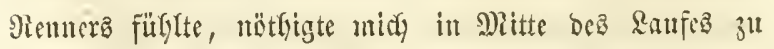
einer Betwegung, bie ifn parirte (zum Stiffefyen bradte). Der Sirall, ben idf baburety Gefant, marf midy beinalye über jeine Shrem; und Bott weis, meldyes Gelädyter wuter ben Srabern entitumb, die biefen Itnfall mit anjalyen! 
Эa) war nidyt im geringften erforedefen, unto ber Mitt,

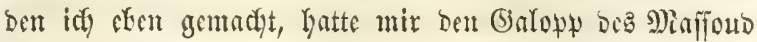
fennen gelefgrt; mu mollte idy aud fornen Trab nidft unverjucft lafjen. Dieje bangart war ign gemiffer=

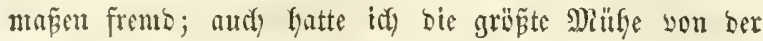
Solt, ifn in ben Trab zu bringen; was mir aber bod

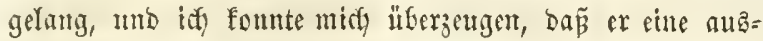
gezeidynete Edulterbermegung Geją̧. Seerr won Fortes fteigerte aljo ein wenig fein Siebot und willigte enblich in ben Freis, welden Der 2raber verlangte. 2rber als

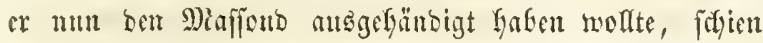

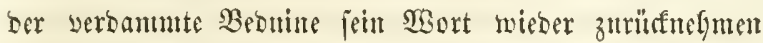
zu wollen, insem er jagte, baf er nody unter bem Titel cinç Bactfiz (Gefthenf) bie Beinfleioer bes Serm yon Bortes Gefommen milfife. Dicjer Forberung mar fotwer zน genügen, benn bas verlangte Nilcionngşftür mar baz

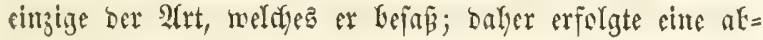
fdylägige 2rntwort, bant ein Grreit. Suleżt fam man jebory üfer ein mezzo termine üferein. Man fajübte baz Scinfleio und Der ftorrige 2(raber erbielt beffen (B) lonerts.

Sicic man gejelyen, fam aljo ber Mlafijoub nidyt obne

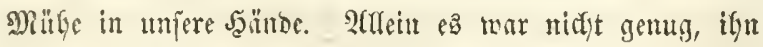
gefurt zu Gaben, fonbern nix mus̈ten anch baranf Denfen, ifu ber Siatigier unferer Freutbe, ber Straber, zu ent=

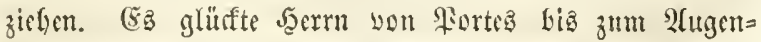

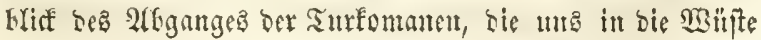




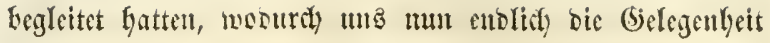

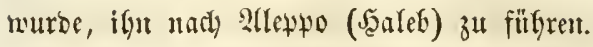

Dieje Romaben waren Befther cinter grop̃en 2 tnzaly Rameele, bie fie yon bem Traberfanme Foebant und anberen gefauft batten, weldye baut alle Jafhre, went

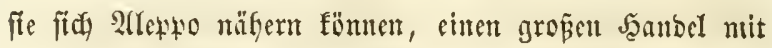
ben Turfomanen ber Šbene 2(ntiod)iens treiben, bie bie Furdft, getlündert ju werben, abgält, fitch in bie Mitte Der Silifte und unter bie Bebuinen zll magen. Nidy, ctwa, bá bie Turfomanen felfft feime Rameele aufzögen; benn obgleid bie ifyrigen foüöner und grëper find, als bie ber Mistife, fo find bie regeteren boch meit melyr ge= affet; wesffalb bie Turfomanen audi nicht aufgören, fie

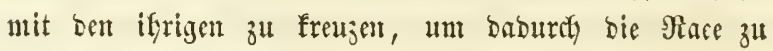
werbefiern. Der Srets biejer Thiere ift nidyt hod); ife

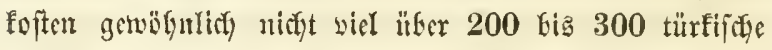
Fiafter (150 bis 225 Franfers). Demungeaditet merbent bic rementon Dromedare ober Simede ju 1000 un 1200 Fiafter (750 bis 900 Framfen) werfauft. (Eit ober zwei Tage yor ifrem 2 tggange Gatten umere Turfo= manen alle thre Ranteele um ifgre Belte verjamutclt. Dieje Borfersitungen waren iebod nidjt io gefyeim ge=

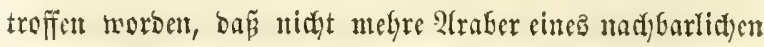

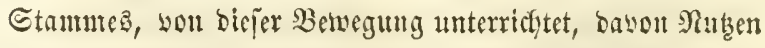

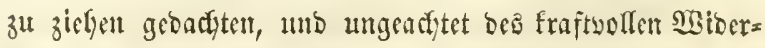

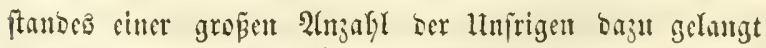
maren, fith in unjerem' 'ager Sutritt jut veridyafien unt 


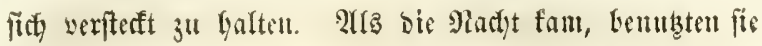

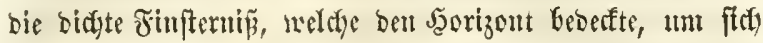
bis in bas 2 ager ber Turfomaten z" jajleidfen und biejent braven Senten brei Samedle, ein Stutfïllem, Turbane, Giürtel unb eine Menge anberer Gegenfitänbe zu fteflent. Diefer Diebftagl müroe nody weit Geträditficter gemejen fein, wenn man cinem einge(d)lafenen Türfen nidyt ben Turban, Der ifm ben Sispf beberfte, weggenommen lyätte,

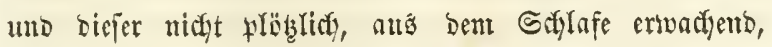

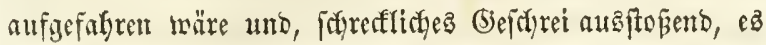
nicht bafin gefradyt fyätte, nadi biejer Seite Gin bie 2raber umferes Stanmes herfeizurufen; aber foron mar

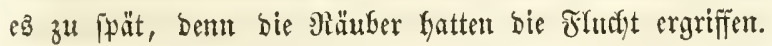

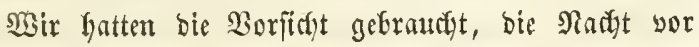
unjerem Belte, über unjere 2\{nfäufe wadbens, zujubringen; unt wie gut war $p$, fo wardjant gewejen zu, jein! bern

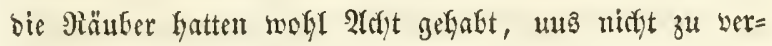

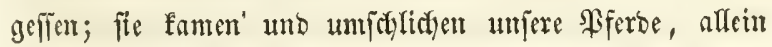
unjere Gegenwart wereitelte alle ifre $\mathfrak{B e r f u d y e}$ unb nötfigte fite, anberwärta ifre Sülgnfeit uno Talente zu verfucthen.

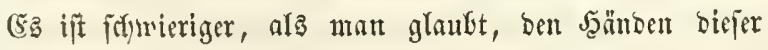

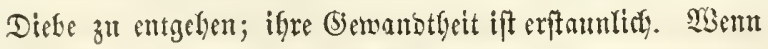
fie bergletdyen Borfiaten ausfülyen wollen, wällen fie

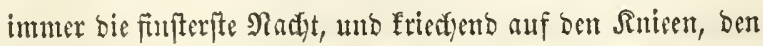
Sä̈nden oder bem Bauthe fommen fie in bie Mitte einez

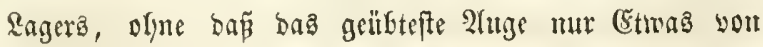
ifyen Betregungen wahrnelynten fann. 
Den folgenden Miorgen begaben ftry Der Sryeit, feim Bebeimfryreiber unb einige ber vornefmetten 2raber bez

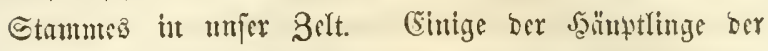
Turfomanen faumen ebenfalle; es Gandelte fith bet ifnen Darum, Dent Dufaï von jebem erfmetten Sameelfowfe. cin Bardits zu zablen. Die lliterfaltung, wie mast fits

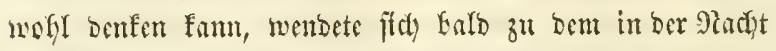

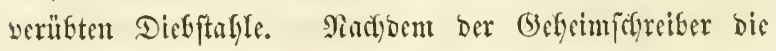
Rlagen ber Tutomanen rubig angefört Gatte, fagte $\mathrm{er}$, um ite zut tröften, bas, menu fie cimwirligten, für jeben

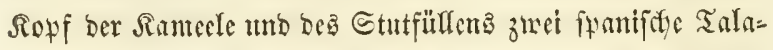

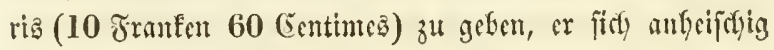
mactete, ifunen biefelben Gerbeizujefaffen. Diefez Beripredten mowiber idf anfangs erftaunte, war jesody für meniger

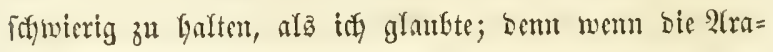
ber cinen Diebjtabl begelyen wollen, jo mäblen fie fith zu

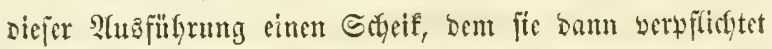
find, alle gef́toblenen Sachen einzulgänbigen. Seţterer nimunt nun eime gleidye Theilung unter ben Theilnelynern yor, welde ofne bieje int Eimzeluen gewaltigen Serrectumngen

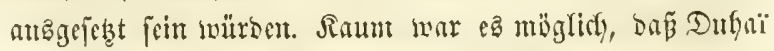

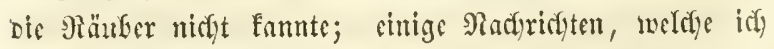
feitbem exfielt, madyten midy fogar glauten, dã er mit Denfelben unter ciucr Derfe war.

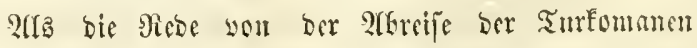

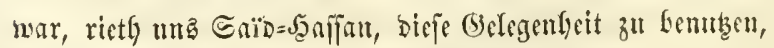
um bie beiben \$iferbe in Gidyerbeit zu tringen, beren 


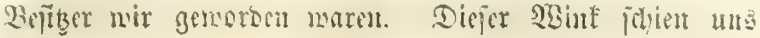
unt fo verfändiger, Da Miaffoud ber Gegenftand lebgaften

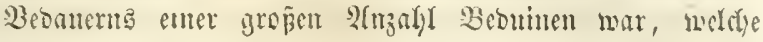

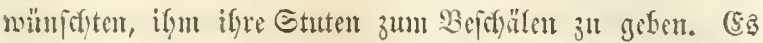

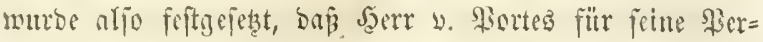

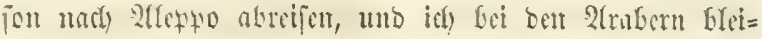
ben follte, fits auth ich eitue tafjente (belegentyeit fümbe, baffelfe zu thun. Dod), Da bor Marict cimer Sornmane mit Someden jefr langinn mo bafer Inngweilig ift, glaubte ser Stalfmeifter, um Beit zu geninmen, beffer zu

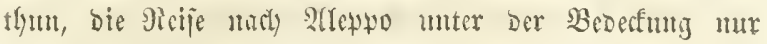
eines ber 2trufur Dufgaì zu mathen. (Sr ging aljo zum

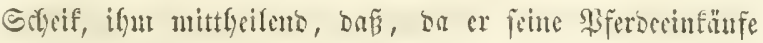
fortjetzen molfte, aber unjere Mittel fith zu werringern aufunzent, er fich vorgenommen fä̈tte, nad) Slleytro zil gefen, und bat ifyn bemuad), ifju einen feiner Sieiter zur Begleitung nitzugeben. Da Dufnii einwilligte, hatte num

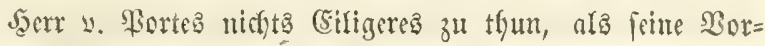
bercitumgen zur sersife zu treffen.

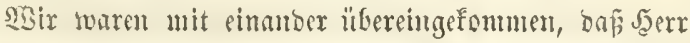

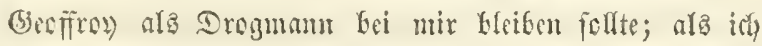
ibn num won biejer Beftimmung unterridstete, erfyob er cill grofeses Seforeci, inbom er fagte, daj er abreifen molde und ifu Siduts in ber $23 e f t$ zurüfforten würbe, länger unter biejen Barbaren zu bleiben, neldye firth jeden 2fugenblift au unjerem \&eben vergreifen fünnten.

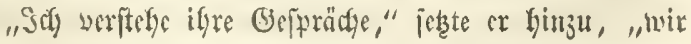




\section{4}

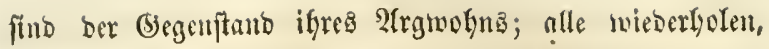
Dấ mir megen gamz anberer Dinge in ifgre Mitte ge=

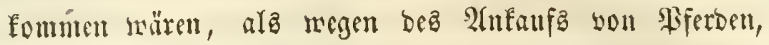

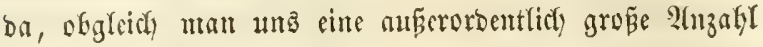
gezeigt fyabe, wir ignen bod mur żmei afgetaufut Gätten.

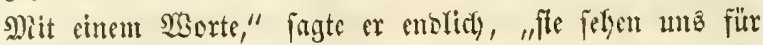

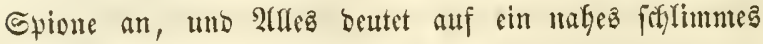
(Ende."

"Nun gut," ermicberte idy, "rcifet! afer idi Gleibe mit Breorg."

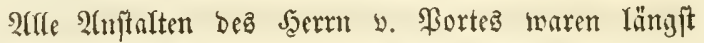
getrofien, und bod fam ber uns von Dafgai verffrodyene Sieiter nidut. Der Stallnteifter, ungeduldig afzutreijen, erjuthte mith, midy nady Den Hrijacten biejer fonberbaren Bögerung zu erfutbigen. S(t) begab nich) alfo zu bem Styet umb fand ifgn bet felfr follectyter saume.

"Der Emir=?lffyor mill mich werlaffen, ofye mir zu zahlen, was er mir forduldig ift." Dies mar bic ganze 2(ntwort, bie id) won ifju crfbalten fonute.

(Elye wir you 2 Treppo abreiften, entricteten wir bem

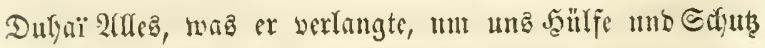
3n ftotern; anzerbem hatten wir ifm nod 300 Piafter (225 Franfen) als Bactitis für alle Pferbe gezalylt, bic

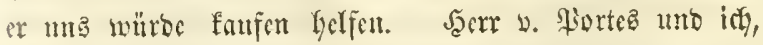

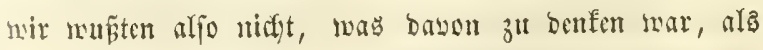
Der Ectjeif pribglidy in umperm Belte eridjien und unz

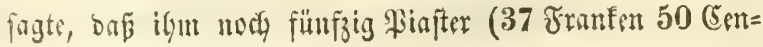


times) für leben Siferbetopf geföorteu, welcher yon unz angefauft worben wäre, fowie noch cin Bacdyis fït bas

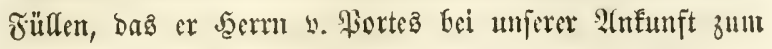
Gejodenf gemaclyt bätte.

Da wir nidjt bie Ctäreften waren, mufiten wir alpo

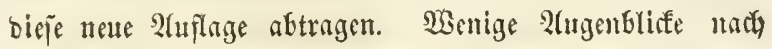

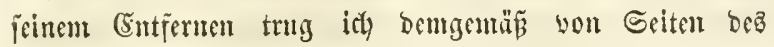
Serrn ๖. Fortę 150 Biafter (112 Franteen 50 Čntintę)

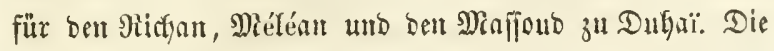
fidnelfe Berïnderung, bie ity in bem Betragen beffelfen gegen uns bemerft Gatte, beumrubigte mich. Snoem idf nun meine Genbung feierlidyer mathen uns ifym melye 2lettung gegen unz aboringen mollte, exidjien idf wor iffm uit jwei Dingen werjefyen, mit benen wir nns vor unjerer 2abreife won 2(replo zu verjorgen fïr nötyig gefunben Gatten. Das cime wax cin Brief yon Rurjyid, bnz an=

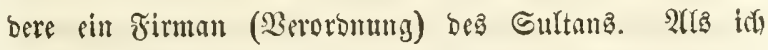

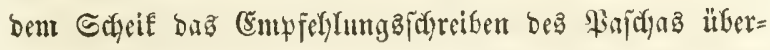

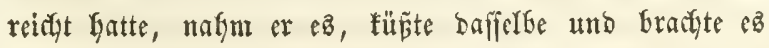

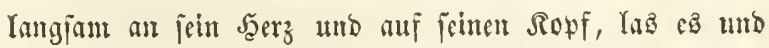
ftelle mir baffelte wieber zu, matyden er biefelben Betwe= gungen wieberbolt hatte, bie idf fo eben mittheilte. $\mathfrak{X B a}$ ben Firman bes (Brop̧ferru antectraf, fo zeigte Dufyai bei bem Eumpfangen beflelben biejelbe Ělyrurtyt, las ifyn mefren amwefenden 2 trabern Iant vor, barauf ïbergab

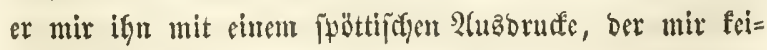

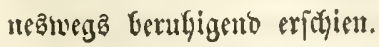




\section{4}

find ber Stegentanto ifres ?trgwohns; alle mieberbolen, Daj wir wegen gally anberer Dinge in ifre Mitte ge= fommen wïren, ols megen bes 2lnfaufs non Siferben,

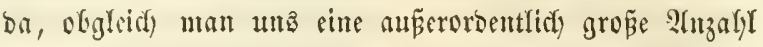
gezeigt babe, wir ifnen boct mur zmet algefaruft bättent.

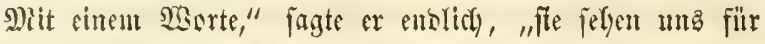

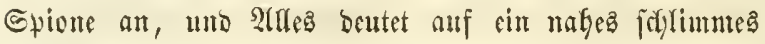
(E)tre."

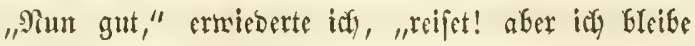
mit (Bieorg."

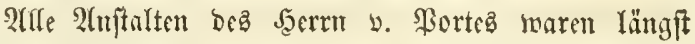
getroffen, und body fant ber uns von Dubai veriprodyene Sletter nirgt. Der Stallmeifter, ungeouldig abzureifen, erịudte mish, midy nady best lirjadjen biefer jomberbaren Bögerung zu erfunoisen. Sart begab mich aljo zu bem

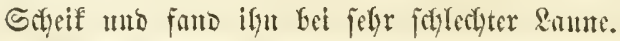

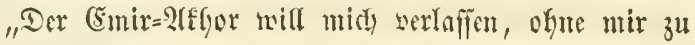
zalylen, was or mir foduloig ift." Dies mar bie gauze 2ntwort, bie ici) vou ifym erfalten founte.

(E) we wir wou 2lfeppo abreiften, entrid)teten wir bem

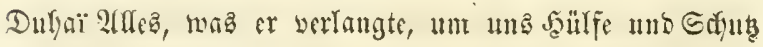

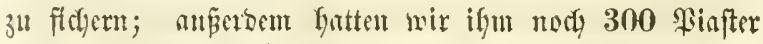
(225 Tranfen) als Bactio für alle Siferoe gezalylt, bie

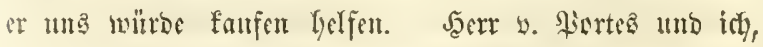
nir muften alfo nidgt, wa bawon gu benfen mar, als

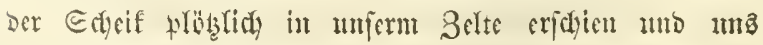
ingte, ba仿 


\section{5}

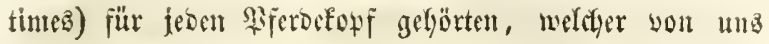

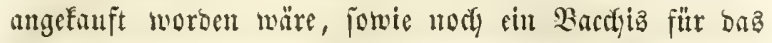

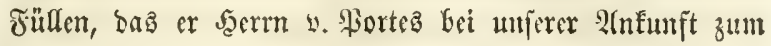
(Beichenf gemadyt liätte.

Da wir niç) bie હtärffiten waren, mušten wir aljo

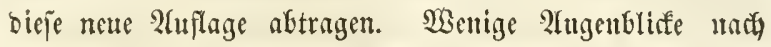

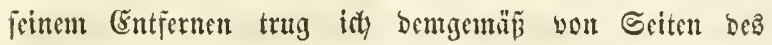
કૃerrn v. Fortes 150 Piajter (112 Franfen 50 (Eentimes)

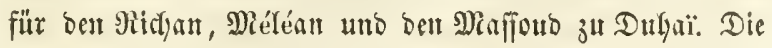

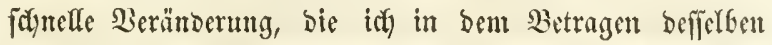
gegen uns bemerft hatte, beummbigte misty. Snbem idf mun meine Sendung feierlidfer madyen und ifgn ntelyr Ildftung gegen uns aboringen nollte, eriffien ide) wor ifgu mit z'we Dingen verjeffen, mit benen wir uns yor unjerer

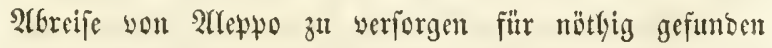
hatten. Daz sine war cin Brief yon אurrajcio, bas an=

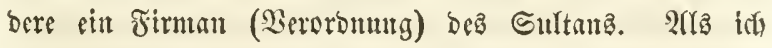

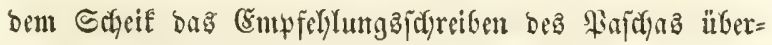

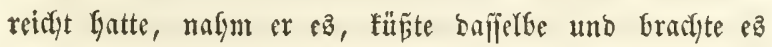

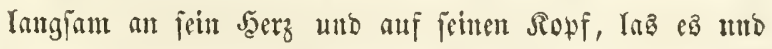
ftellte ntir baffeltbe mieber zu, uadjoem er biejelben bene= gungen micoerfyolt batte, bie id) fo eben mittfyeilte. SצGa Den Firman bes Grobgerrn aubetraf, fo zeigte Dufaï

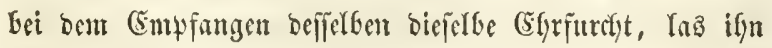
mefren anwejenden 2 (raberm laut vor, barmif itbergat

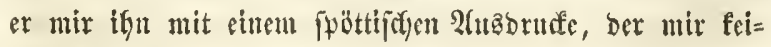

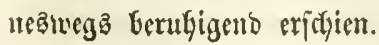




\section{I36}

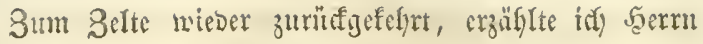
y. Sortes bort ganzen Borgang. (Er beptinme fogletit),

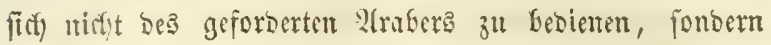

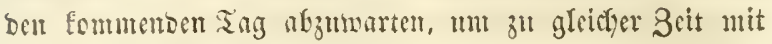
Der Sarnmane ber Turfomanen afzutreifert. Diejer Ent=

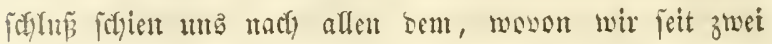
Tagent Beugen gerrefen warew, unt fo geratgener $3^{\text {ut }}$ jein,

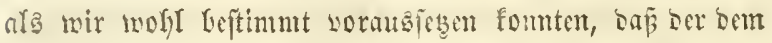
Jeern v. Fortes mitzugebende füfgrer den Befefrl era halten hatte, benjerfen in cincen feinterbaft fallen za laijen

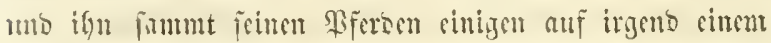

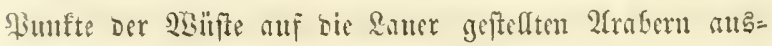

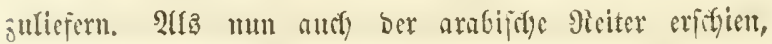

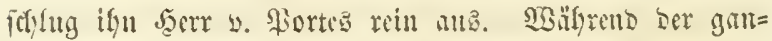

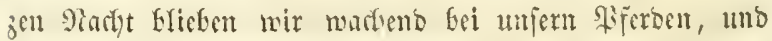
bieje Sorfint war nidgt umötbig, Sem ç gab nicberum

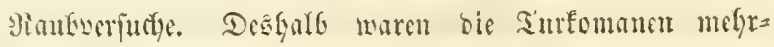

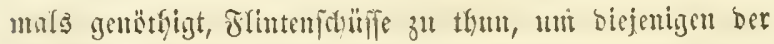

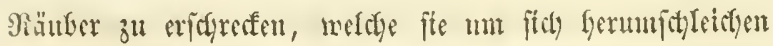
rörtert.

Dent anbern Tag, uartbem mir ber Stallmeifter

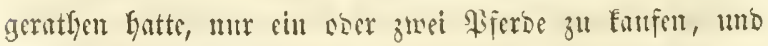

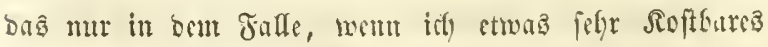
funden mürbe, begab ar ïtr) nach Somtenaufgang anf ben

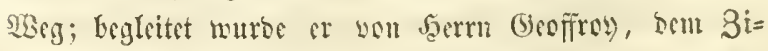
getmer unb von Miegrediffe, jenem ?(rmentes, ben ber

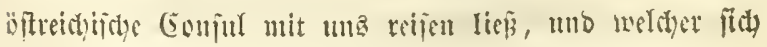




\section{7}

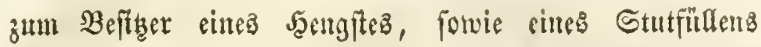
gemadyt Gatte. $\mathfrak{A H A}_{e}$ brei ritter auf ber Saramane gefgörenben Drontebaren und verlięét bas হager, ins bem fie bie Pferbe, weldye fle unit fortbringen wollen, zutr Seite filfyrten. 


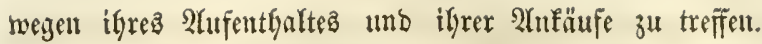

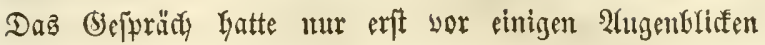

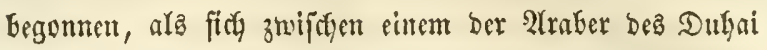
unt einent ber Neunangefommenen ein to heftiger Streit

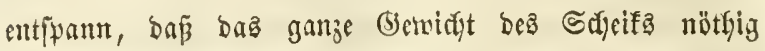
war, unt ben 2(raber zu werbindern, feinen Gegner zu

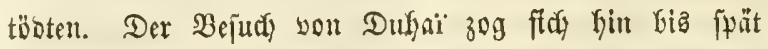
in bie গadyt; er werlię̧ midy, inbent er bie wärmiten

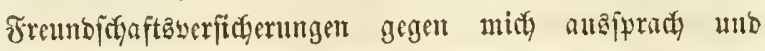

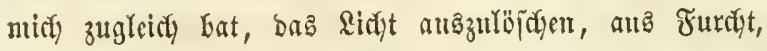

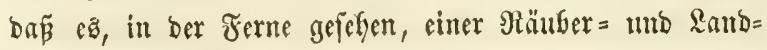
freitbertruple zum Jülrer bienen fönne.

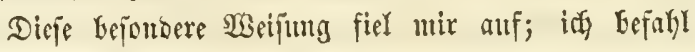
batyer bent Georg unb bent Bigenuer, weldye Saert v. Portes ntitgenommen, ber fich aber beeilt batte, mir

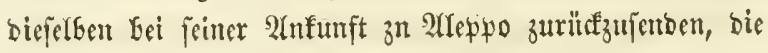

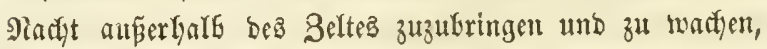

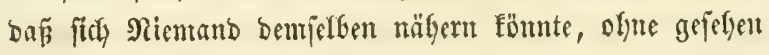
zu werben; ich felbpt nafut mir vor, biz zum Morgen

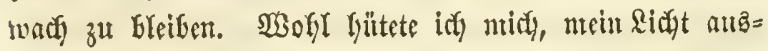
zulöjden, gebraudyte aber bie Borftdyt, es fo zu ftellen, Daß̄ man

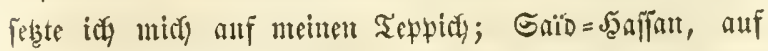
bem feinen liegents, fdylief neben mir. Die tiefe Stille int Selte iwurbe nut burdy das buntyfe und gebebnte (5e=

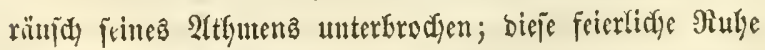

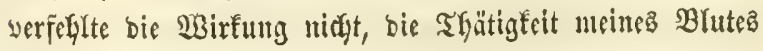




\section{1}

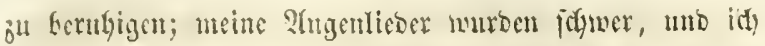

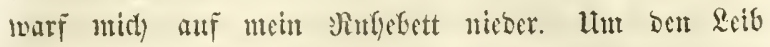
batte ict) cinen (sürtel, in meldfem neine Piftolen feteftent. Da mict) ater ber lunfantg bieję fremben

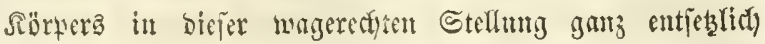
beläitigte, entlebigte idf midy beffelfen, fomie mentes

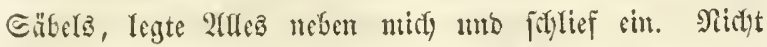

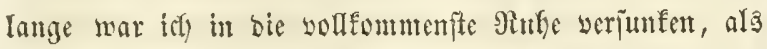
nitid ein Flintenjetur in ber Bitutung nad) bemt Lager

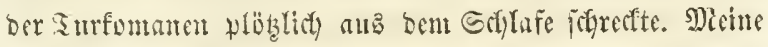

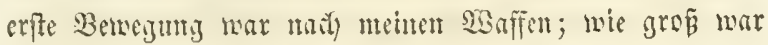

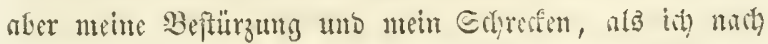
lontgen Suthen berfolfen feine Spur won ifjen ent= Derfte! Sch frrang aus bem Belte, werfite bicorg mo

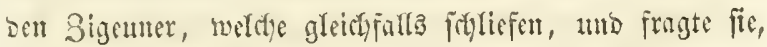
ob fie nitht meine Sasuffen forttragen gejelgen Gätten.

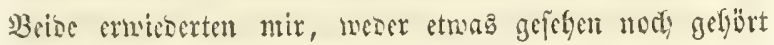
3น haber.

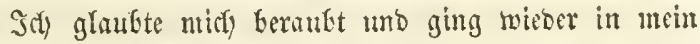
Belt, won bom Bebanfen Gommulyigt, Das man trob all' meiner $13 a d j$ anfeit bod bis zu mir gebrungen fei, als

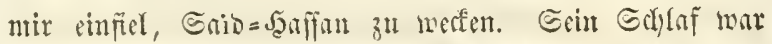

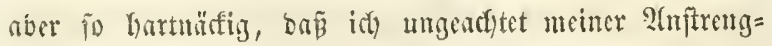

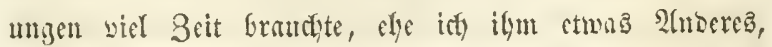

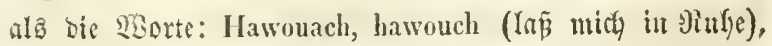

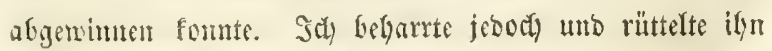

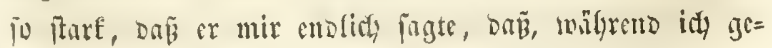




\section{2}

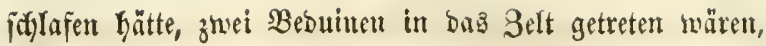
und ba fte bie gänzliaje Sithe bemerft, welcte un midy gefjerridut, fowie meine $\mathfrak{W a \pi ̃ e n ~ e r b l i c f t ~ G a ̈ t t e n , ~ b i e ~ z แ m ~}$ (S)brand) סеכ Erpten, Bepten frei bagelegen, fie dief̧elben aufgefoten und unter feinent Teppidy veriterft bätten. Saf) flog foglcidy nady bem angezcigten Srte und fatte bie Frende, bie verloren geglanfen Gegenjtänbe wicber zu finben. Num aller nteiner Befïrdftungen entlesigt, freste idy midy you neucm anf ben Teppich uno fablief olye Störung Gis zum Miorgen.

Bei mcinem (Erwadyen famen zmei 2raber zu mir,

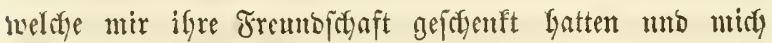
jeocn Miorgen mit Mild won ifren Rameelen verjorgten; biefe beridyteten mir, baß́ jie ę jelbft gemejen wären, weldye mir ben mädytlidyen Befudd, ber mid fo felyr be= unrulgigt batte, gentacht bätten. Gic maren bei meitem Selte worübergegangen, und ba fte bas sidat burdy bic Lcimmanb forjumern geflefyen lyatten, waren fie eingetreten,

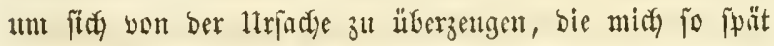
nody wadyen lię̧e. Da fie midy aber foblafend gejumben,

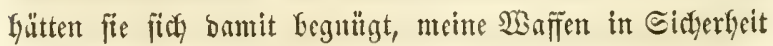
zu bringen. Diejclben fügten bimzu, Daß fite beim Seraubs= geben won cinigen Turfomanen bemerft morben mären, morauf benn bieje neuten 2 (nfömmlinge, fie mabridjeinlica fïr Эiänber Galtend, auf jie gejdyoffen Gätten.

Das Bebauern, welidjes idy biejen Graven 2 enten über bic (befabr bezeigte, ber idy fie ausgefetgt hatte, war 


\section{3}

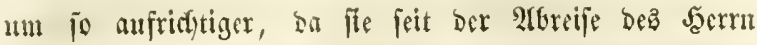

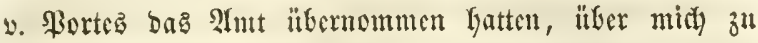
wadyen uto midy son alfent ben Eleinen böjen uno heims

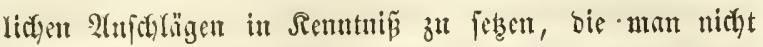

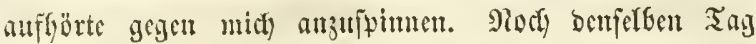

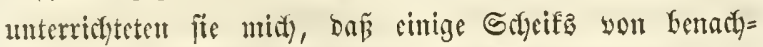
Garten Stünmen bem Dufgai einen $\mathfrak{2}$ (nja)lag mitgetfeilt

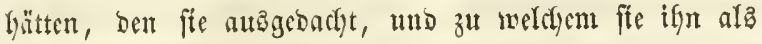

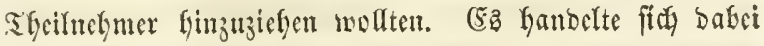

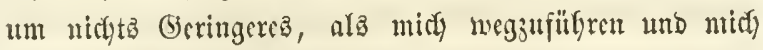

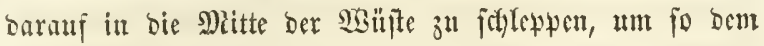

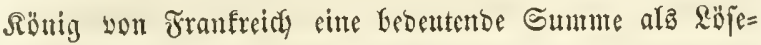
geto abjuforsent. Duffaï, fïgten fle limbu, bätte aber biefen $\mathfrak{A}$ ntrag rmmb abgeĩalagen.

(Grof̧e Mafien yon Rameelen hörten nidyt auf, im Rager anjuftommen; joren Morgen fauften bic Turfomanen

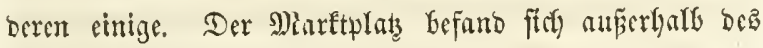

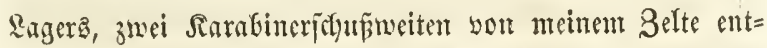

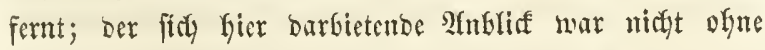
Snterefie für micly, aud bradgte idy bufalgft feben Tag einige Stunden zu.

(ii) Gejauto mich aud fict, als cines Tages ber

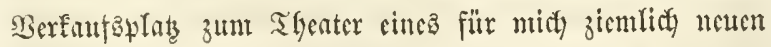

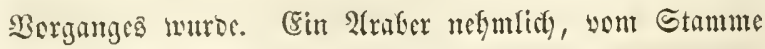

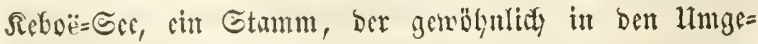
bungen yon Bagbab lagert, batte fita ciner perifitityen

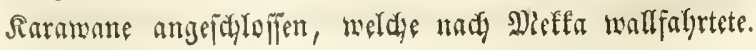




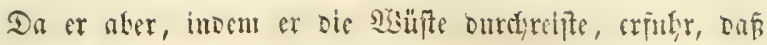

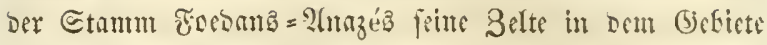

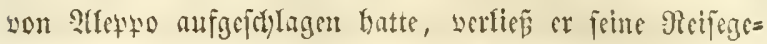

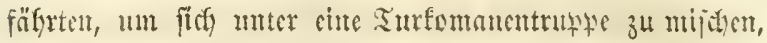
bie, wie or mufte, zum Ramedmarte ging, weldyer im Etaume dez Dufaï afgefalten wurbe. Itnter ber Bereleibung eines arabijcten Rleiberbänolers latte er nun nidyta ciliger zu thum, als fict bei saten, ocmen er be=. gegnete, zu crfundigen, of ilynen niclyt in sager ein

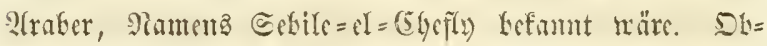
gleich er nur vetneintende Antmorten erbielt, licé er bcu= nod) ben S)luth nicht fitnen, tmb ofue Somtanden bon (Grumb frimer 9 तad) forjdungen zu wertrauen, fulle er barin mit mermübeter Tgätigfeit fort. Den folgemoen Tan, jebr früh, war or mieber auf bem Derfanfoplate, feine Fragen bez worigen Tagez mit ebcnio menig Grfoly cr-

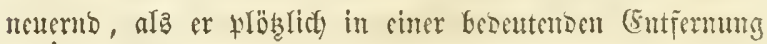

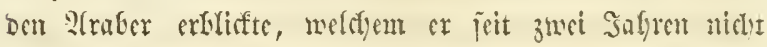
angehört batte unter allen Brlten ber Sibinte nadujn= fescren. Den Gibel ziefyen, auf feinen Frinto mit Der

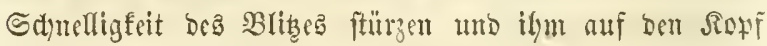

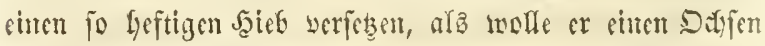
forlten; alles biejes war bie That mur einiger Gefuntor. Sndent er fith frinem Dyfer nälycte, war bie SButh in allen feinen S̈̈̈gen angenrägt, แmb als cr bent Etreict)

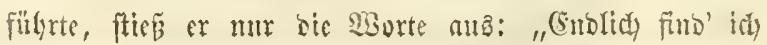

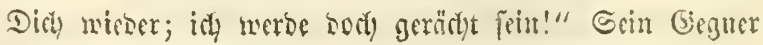




\section{5}

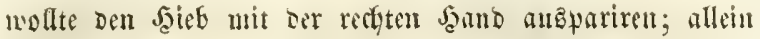

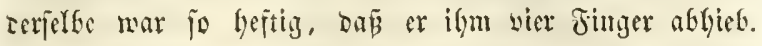

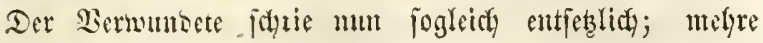
Araber, auf jein Sicfdrei ferzueiłent, ftürgten uber ben 2tngreifenben her umb in eitrem 2tugenblife waren eite

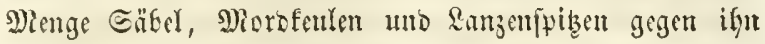
gerid)tet. จ(nd) würbe ex unterlegen fein, wenn ę ifym ridgt geglinft märe, eineu Tyeil oer 2(raber, bie ign an=

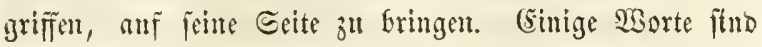

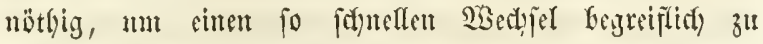

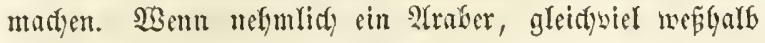

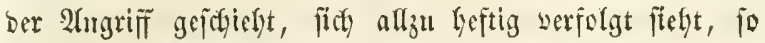
fann er bie Angreifenden jogleich won fids abbalten, wenn ex an einer ber Gdyuren, bie bem Ghanl als Jranjen bienen, meldyen alle auj bem Sopfe tragen, uto ben fie Siefee nenten, cinen Snoten fnüpft. Die ganje Edjuie=

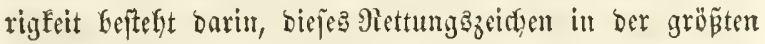
(5efdymindigfeit madyen zu fömnen. Ditten iut (Befectyt, ro ein cinjiger Mann gegen zefur ftrcitet, ift bie Sadye Durchaus nicht Yeidyt; aber hat ber Angegriffene bas (5)lüt, Den Rnsten zu Stanbe zu bringen, fo verändert fid da bas Theater, und jeoer 2ugreifende ift pogleidy Jülfe umb

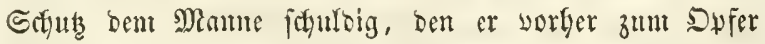
feiter 9 Suth zu madyen ftrebte.

Raum war $e$ E Dem $\mathfrak{A}$ raker gefungen, ben heiligen Snoten zu fülpfen, als ftch ein Theil Der (Siegner auf feine Eeite ftellte, jebody obue gegen bie 2trafer zu fert)= 
ten, you benen fie fidd getrestnt Gatten; fie Gegnügten fid santit, bie auf iffren menen Edyübling geridyteten Şiebe auşu pariren utno ify fis in bas sager zu begleiten; fhicr eimmal angefommten, veriffaritten fie ifm bie Belegentyeit,

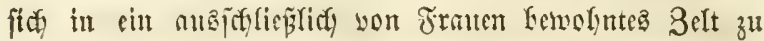

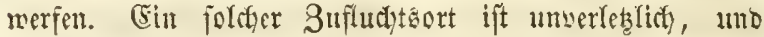
ein 2(raber ift milyrento ber Beit, ala er Gier Glciken fam, wolfommen jitiser.

Der ltuglürflict)e, welefyen ber Flürtytling berftüumelt

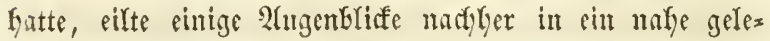
genea Belt. Bei jolden Fillen, nie bicjer, ben idy fo eben mitgetgeilt habe, fatreibt ber Gefraudy oer Säufte

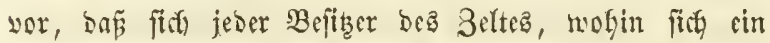
Feind gefliteftet hat, ber Angelegentyeit peines Gajtez an=

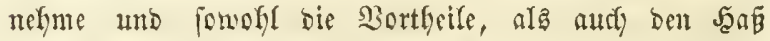
Deffelten theile.

Diefes 2lGenteuer serfreitcte fith bald im ganzen

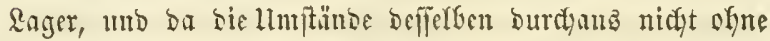
Sistintigfeit marem, bejffico Dubnï bie 2llten be Etam=

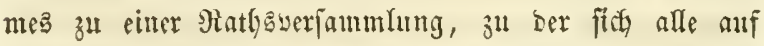

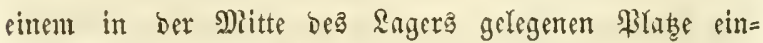
fandent. Saier Girbeten fte ein Beriagt unter bem $\mathfrak{B}_{\mathrm{or}}=$

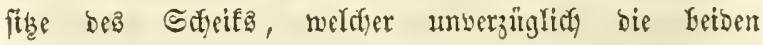

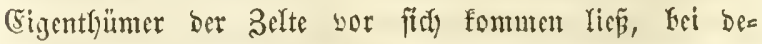
ten feber ber Geiben ITraber 3ufludyt gejuctit hatte. Die Sişung fand unter fretent Şimmel in ber $\mathfrak{R}$ itte einer grofen Miaĩe 2 trafer itatt weldue fith im Sreife 


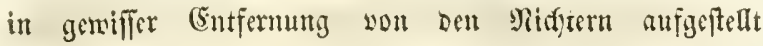
Gatten.

Die geridgtlidye 2lnflage murbe won bem Sisitlye bes 2trabers, bent die Finger abgelyamen waren, vorge= tragen. Scin Siegner antwortete ifm, inbem or bie Sirünbe barlegte, meleye ofn fremben $\mathfrak{Y}$ (raber zu einem fortien gemalttbätigen 2lugriff betwogen batter. (5r trug

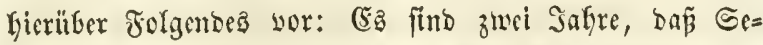

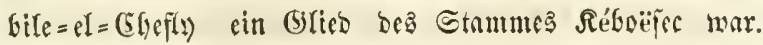
Sine Raramane wurbe augeplïnoert und ar hatte 2 (n= foruch auf cinen Theil ber Beute. Bei ber Theilung entifanten Etreitigfeiten mit ben 2(raber, ber ifn eben

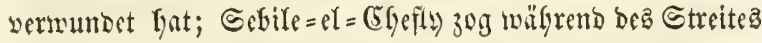
fcinen Säkel unb zerlyieb meinem (5)afte anf ben eriten

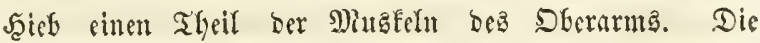

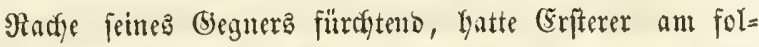
genben Tage ben Stamn berlaffer. Das Blut meines Gajtes mar geflofien; basjentige bes Eebile mufte. alfo nun bas feinige bezabyen; Das ift, wie ifr wip̈t, daz (S)efeb ber Wieberwergeltung! So wie er gelyeilt war, verlié̃ mein (Saft friu Belt und machte fich auf, Gebile aufzujudyen. Șälyremb zueier Sabre bejuctite or mu faft alle Ctämme ber פäufte Syriens uno begegnete erfit Gent Niorgen feinem Frimbe. (5r hat bas Bhat zurüf= genomumen, meldyez Eefile von bem feinigen vergoflen

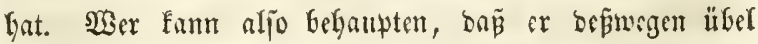
getyan Gabe?" 
Derjenige, welther bie $\mathfrak{A}$ raber femut, wird biejen

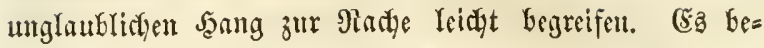
fteken unter einigen Fantilien Siriege, meldye loft Yänger als ein Sagrtyundert bauern; bemn fo lange es nodi zmi=

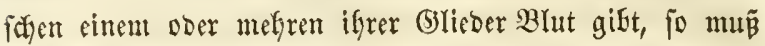

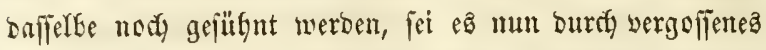

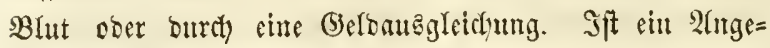
griffener geftorben, ofyne jeine Estullo afgetragen zu haben,

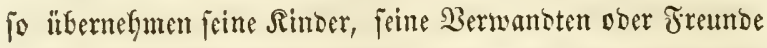

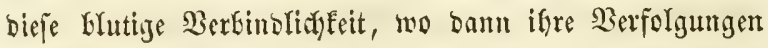
nicht cher alffören, alz bia fte yolfonmenen Erians

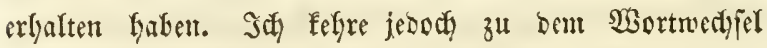
zurünf.

Der Wirth bes Sebile antwortete feinem Biegner,

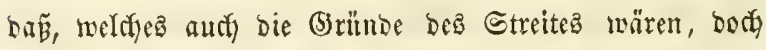
bie Bebettenbleit ber Sisure affemal cine Benugthumng

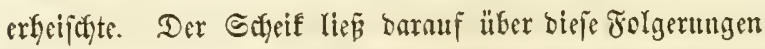
abjtimmen; fie murben angenommen unb bie Ricteter bes

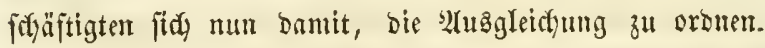
Der Rläger verlangte für jeben afgethauenen Finger zmanzig Sameele; Das Tribunal erflärte fit bannit einverftanoen

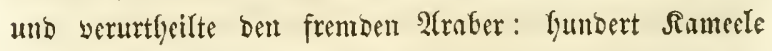
an Eebile zu geben. Da mir mun Georg Eat für Enk

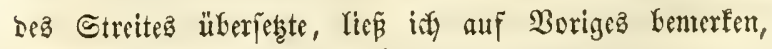

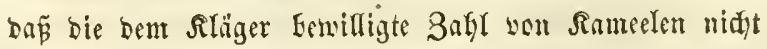

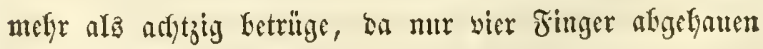

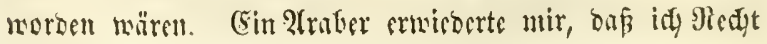




\section{9}

Gătte; Da ifm aber ber allein fteben gebliebene Daumen won mun an feituen Nuken mebr gewäfren fönnte, fo

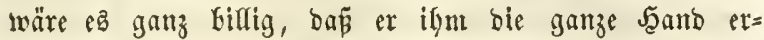
jeben mü̈ßte. Sđ erwieberte barauf nidyta; allein meine

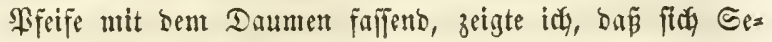
bile beffen noch febienen fönnte, fei cs auth nur zul bie $=$ fem Befraude. Die Rirfter maren meiner Dieinung unb verntuberten un zwanjig Santerle bie Kemilligte Ěntjăbigung. Die Unterjudyung ging Gierauf auf bie

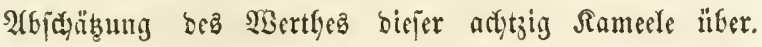

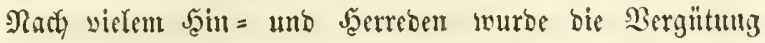
auf 800 Siafter (600 Jraufen) nebft sem Säbel, ber bei ber Berftïmmelung gebraudyt worben war, jeftgefetst,

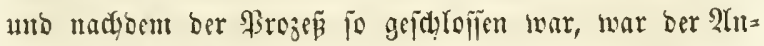
geflagte jeber Berantwortlidyfeit entboben utro freigepiprochen.

Sä̈reno man ben Geldesmerth jebes ber Finger.

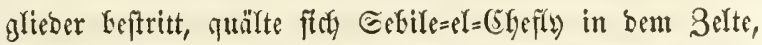
wo er einen 3uffuctionort gefunben gatte, nit ben grau=

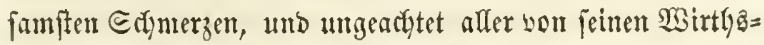

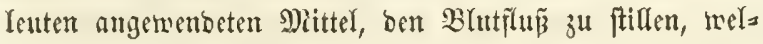
dyer bie Folge ber Berwutung war, werlor er bemods eine ungebeure Menge Bhut. Man war gentöthigt, bic= jenigen Mlittel anjumenoen, zu weldyen bie Araber ifyre 3uflud) nefyuten, wenn ber Falf gerägrlid wirb; man

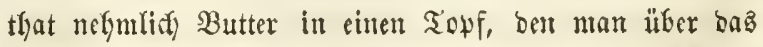
Feuer jetzte; als bieje zergangen war uno zum Sodyen fant, taudste man ben Stumpf biejes yerivundeten armen 


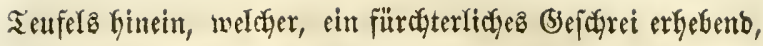

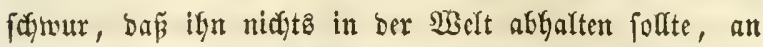
benen Madye zu nefimen, bie ifg fo entifeblich marterten.

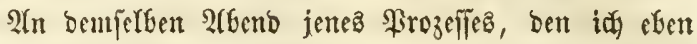
nitgetheilt babe, war idh 3euge cines anderen, nidat mins ber cigenen 2luftritteş; ebenfafle murbe bafei $\mathfrak{B l u t}$ ver=

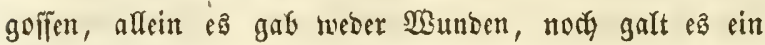
Dienjadenleben.

(Ein Sameel, weldyez bie Turfomanen feit einigen Tagen gefauft hatten, wurbe won einer jo beftigen Seiten=

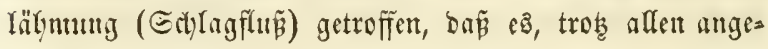
mendeten Mittr(n, zu benen man jeine Suflutift nafm, Doch nicht wieber zum 2 fuffitelyen zut Gringen war. Dafferlese

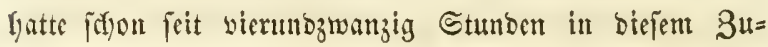

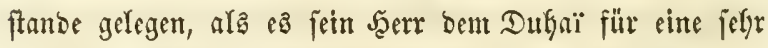

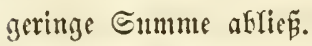

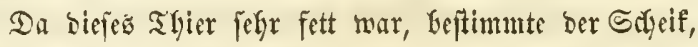

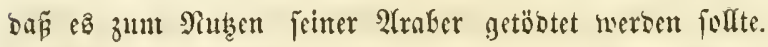
Mian hieb bemfelten jogleit) mit ciner $\mathfrak{2}(\mathfrak{x t}$ ben Sopf ab, umb 2trafer zogen ifym glecich barauj bie Şaut ab;

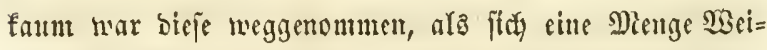
ber uno Sinder auf bie zuffenton Heferrefte beв Thieres ftürzater, indem alle bemilfyt maren, ein Stïrf berjerben

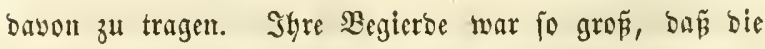

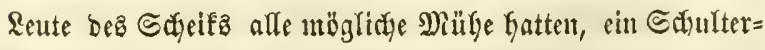

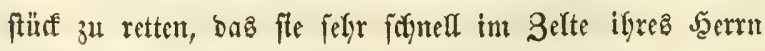
in Sidjerfyeit bringen musten. (sott weié bie 3agh und 


\section{1}

die Gitmere Der Edyläge, meldye wätreno diefes erbitterten

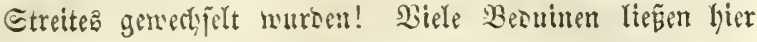
ifre Saare in Den Saünon von Feindinnen, won benen

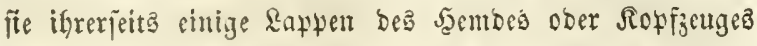
Davon trugen. Georg, melwer fith uneridgrocfen in biefent weiblidyen fantogemenge herumgejdylagen batte, war jo

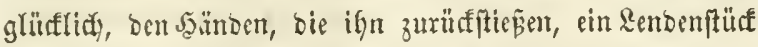
zu entreip̄en, ¿ns ex zu unjerm Mittagsmalyle zubereitete. Die Mänter fatenen bei biejem Rampfe ziemlida gleidy=

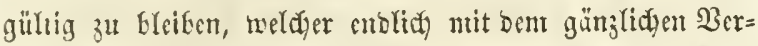
jamminben Des Rameels cnoigte; benn fogar befïen Sno= dyen und Cringemeioe nurben weggeräumt. Mlan fann fid gar Eeime Borftellung you Der Schnelligfeit madben,

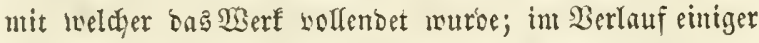
Niunten waren weiter feine Equren bicję biffen Thieres mefr sorfanden, als cinige Blutrfecten, melase son ben Tritten ber Räupfenoen aud nod Galb werwifd maren.

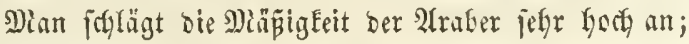

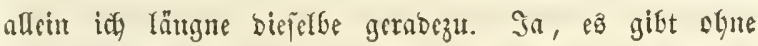

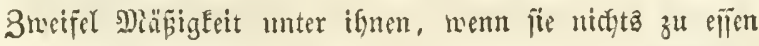
yaben; Denn einige Datteln ober eine Sand yolf Meis genügen ifnen bann einen ganzen Tag; aber, fommt eine Gelegentgeit, wo fie fid volleffen fönnen, fo wirb man

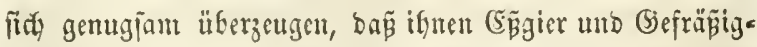
teit nitht frembe Dinge fün!

Gegen Untergang ber Gome begab fidf ber Sdueif,

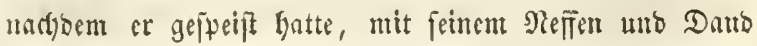




\section{2}

it mein Belt. Derjeffe fam, um feine Redfinungen mit ben Turfontanen ju oronen utb yon ifgen bie Steuer zu crlyalten, weldye ifm erfltidy für bie ifnen gegebente Errtaubriz getgörte, ftty itt feimem Stamme aufzulyalten, ban für bie 2 fggabe, bie ifgm für jeben gefauften $\Re a m t e l=$ fopf zutfam. Dis Turfomanen füunten efenjalls nicht, zu erjedeiner. Dutbaï's Forberungen erregten melyr als

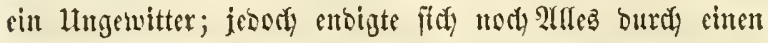
Berglecich, ber burrif bie bienfffertige Bermittelung you

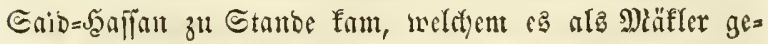
Iang, einen Mittelmeg z"l beftimmen, ber bei allen $\mathfrak{F a r =}$ trien Bifligung fanto, umb bie feitusejetze Eumme murbe an Duffai augagezablt. Die Turfomanen zogen fith nathy her in igre Belte zurürf, ber Edyeif aber Grachte bie

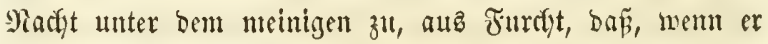

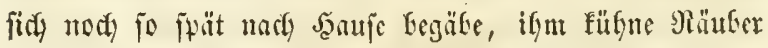
bie empiangenten belojorten abnefymen fömten. ŞSir ma= ren bis zum Morgen inmmer auf ber b̧ut, weil wir jeben

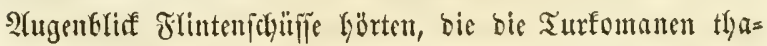

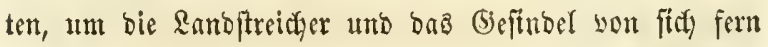
zu Galten.

2(m Miorgen bes andern Tages fall id eine grope Mafie Bebuinen im agager anfommen, weldye alle mit Gtuten beritten waren; fie zeigten mir einige junge Saferbe,

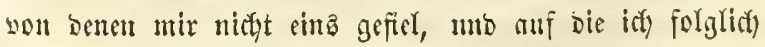
fein befot that. Wfere gegen Mittag erefliffte ich unter

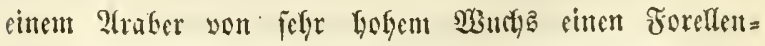


fojiumel, ber mir wegen feiner Jornt, feiner edeln Gangart

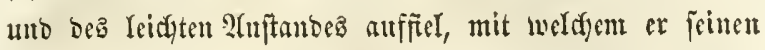

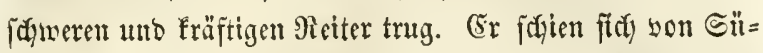

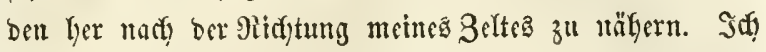
that einige Edyritte, um mir bas siferb näher anjefen

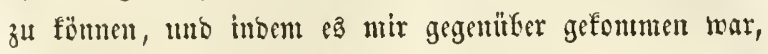

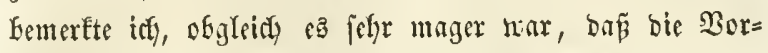
ftellung, trelche id mir wou feiner Sdjönfeit gemadjt

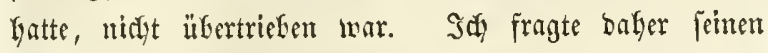
Beftiser, of er nir bafferlbe verfaufen mollte; er antwor= tete mir verneinend und fïgte Gintzu, baß feir \$ijerb von

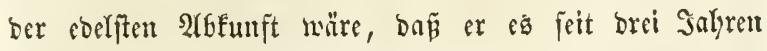

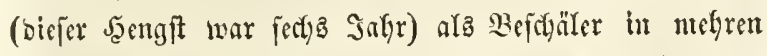

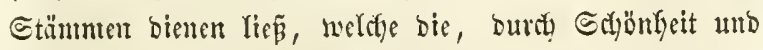

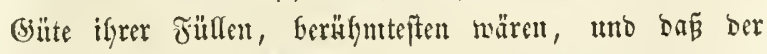
Jengft feit geftern Afend zwei und zwatzig Rienes (elf beutifue Mieilen), um einige Stuten bes Ctammes ber

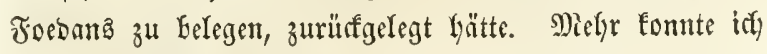
von ifm nicht erfahren; er mentete fein Siferb und ver= jơmanto.

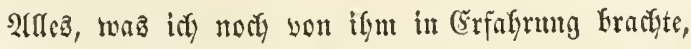

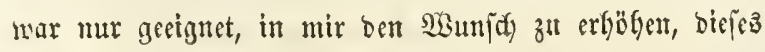
foptbare Thier $z^{\text {tt }}$ bejtizen. Sol fefyrte ziemlitid traurig in

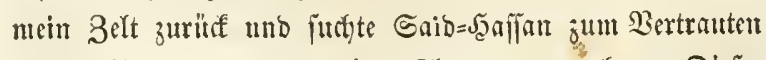

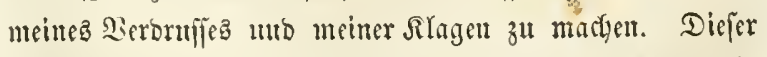

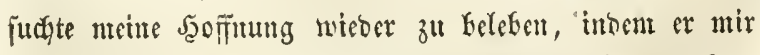

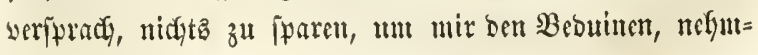




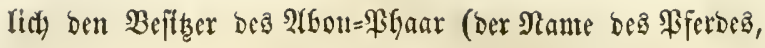

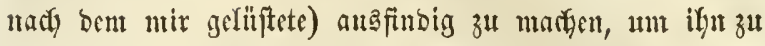

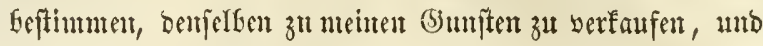
fügte Kinzu: „Miorgen, frim 2hafgang ber Somme, werben

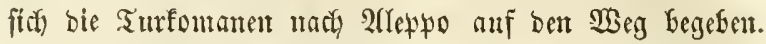

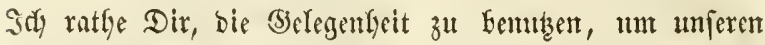

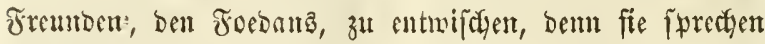

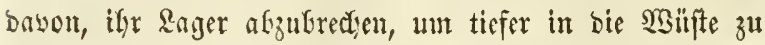

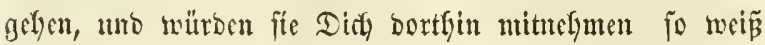

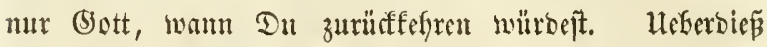

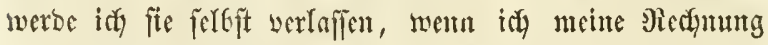

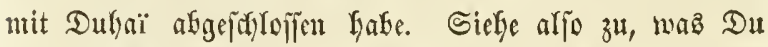
thum millft."

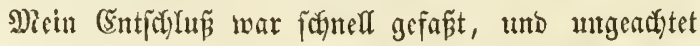

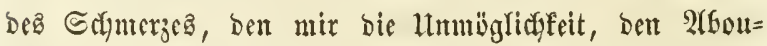

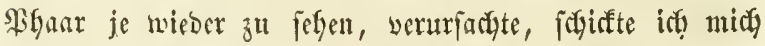
boct) an, ben folgenden Tag abzureifert.

Denfectere 2 fend, bei llntergang ber Some, Gefand

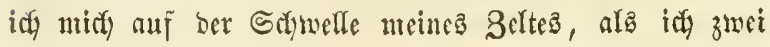

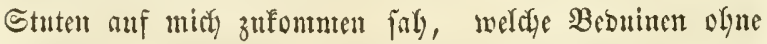
Sattel unb 3aum ritten; cinige Sefunden barauf fal ids

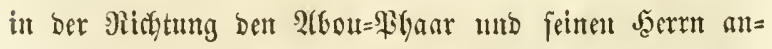
fonnmen. Diejer 2 htrbliaf erfüllte mich mit Freude!

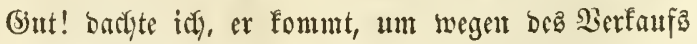
zu unterfyandefn.

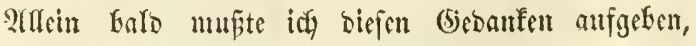

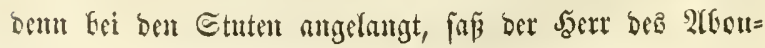




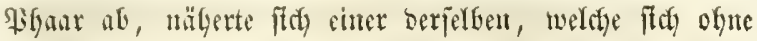
3weifel in ganz rofitgent 3ujtande befanto, und Yieź jue barauf, ofue ifr Spannfeifelt anjulegen, ofne jebe weitere Sorficyt, you feinem Sengite belegen. Nady beendigter

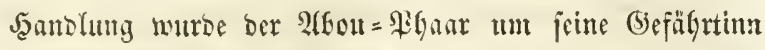
Des 2fugenbliffz Gerumgefilgrt, utto als er fict) weoer in Exection befand, was febr mentig 3eit beburfte, ließ ifn fein 5 err bie 3 weite Gefuringen und biefelbe Sanblung vollziegtert.

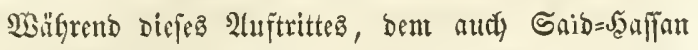
Geimolute, erfugr id, bá ber Araber, beun ber vorzüg=

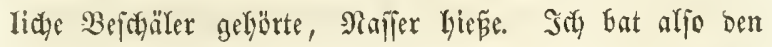
Snib, alles slöglidse zut thun, un ben unlenffamen Safĩer zu vermögen, auf eine Tafje Raf̈ee zu ntir zu font= men. Mein Beltgefährte willigte cin, nifyerte fith bem Araber und überbradte ifyn meine Cintabung, melaye pogleid) angentommen wurbe. Salfer fam, inbem er ben Slbou = \$Gaar an 3aume riclt; chinge anocre Bebuinen, bie fich aud dajelbft befanden, folgten ilym uno wir Sïmmtliche febten uns auf ben Santo. Sa bot meintu

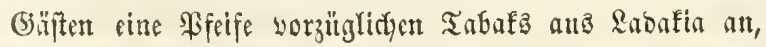
und als fich alle bamit verforgt hatten, 住隹 ich ben Saffee Gerum geben. Die Unterbaltung mar anfangas ziemlida

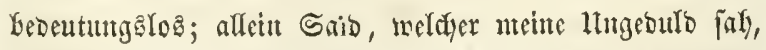

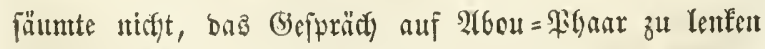
und bent 2traber fräftig zuzureben, mir benfelben zu ver= faufen. Der 2rraber gab lantge Beit gar feine 2rntwort; 


\section{6}

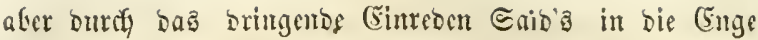
getriefen, Yiéz er entolidy folgente, fo lang erjefnte Baste fïren: "Sage Dein Bifket."

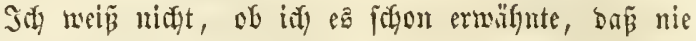
cin 2raber in ber Silüte, mit bent man Gandelu will,

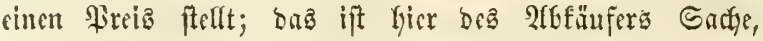

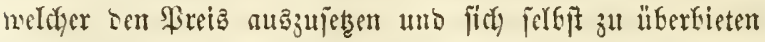

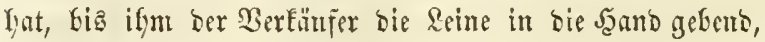

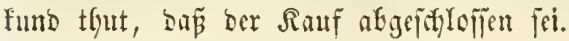

Sady fing bater an 1,5000 Biajter $(1,125$ Franfen)

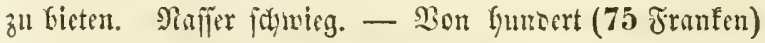

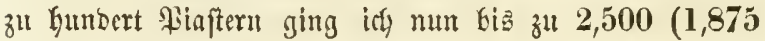
Franfen).

Nafjer, weldyer fitd fis bahin ganz ftill werbalten batte, ftand auf, fprang auf ben 2 fou= \$haar und cnt=

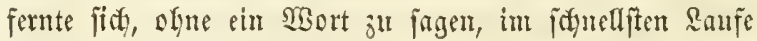

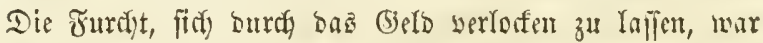

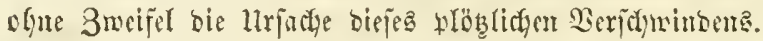

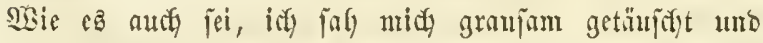
fïrte mägremb ber ganzen Paadjt nidyt auf, bie birlegen= beit zu beflagem, bie idg verloren zu baben glaubte.

In ben 2(bensftumen yatten fitity bie Turfsmamen zur 2tbreije vorbereitet, mo Gaid Gatte won ifnen sin

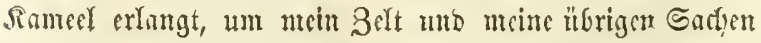
zu tragen. Bon biefer Seite war idy aljo wollfommen

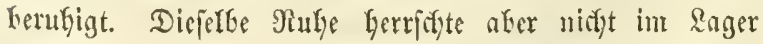
ser Turfomauen, sem mähreno ber giacyt batten fids, 
melye STraber unvermerft, anf Dem Baudje friedjent, in Salfelbe gefdylicien, Gatten bie Sniee mefyrer Sameele be=

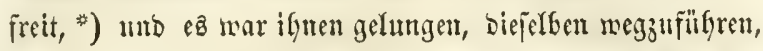
aber bocis nidgt fo junell, um fie ben folgenden Tag nisht wieber geben zu müifen.

Mit 2ufgang oer Sonne liep idy mein Belt $a b=$ bredyen, miafelte in jeine zaflreidyen Falten meinen Man= teljact, nteine Teppiche und Derfen unb jajifte midy an,

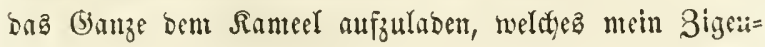
ner Eegleiten folte. Dieje Borbercitungen nafmen meine

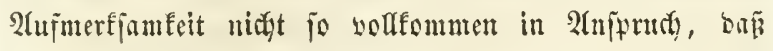
id) uridgt Gätte Gemerfen folien, bas einige ber Sameele, seren Beine wäbreno ber Naryt entfeffelt worben waren, und bie ntan eben zurüffgebractyt hatte, bas sager ber

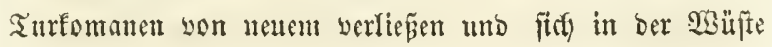
zeritrenten. Die Raramante Gatte zur Beberfung eine

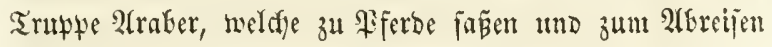
fertig waren; bieje entfernten fitif fogleich wou Rager,

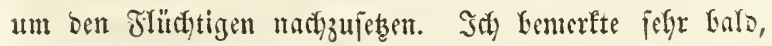

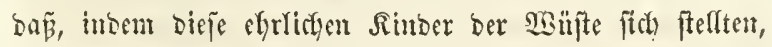
als of fie bie Rameele cinfangen mollten, fie biejelten

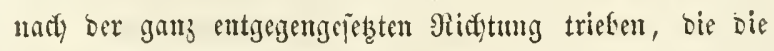
Saramane zu nefumen Gatte; baburch wolltat fie fitg un=

-) Die Araber haben bic Bewohntycit, wälyeno ser siadyt cincz ber Borberbeine ifyer Samecfe zu beugen, inbem fie bas Schienbein an ben Boram binben; fo bas wenn bieje Thiere geken wollen, fie mur brei Beine frei haben. 


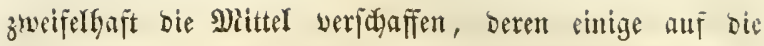
Seite zu bringen. Dieje হift entging aber ben Turfo= manen feineamegs; cine grope 2lnzafyl berjelben fethten fict) auf ifre fiferbe, und es gelang ifnen, nidit olyue Mübe, alle Flüdutlinge zurürfubringen. ख્le bieje fleinen 3wijătnorgänge verzögerten bie AGreije. Duhai, welcher font, un ofne Bweifel bei berfelben zugegen zu fein, war nicht wenig erftaunt, auth nein 3elt afgebrodyen zul feften. (Er fragte mid) mit ziemlicter Syeftigfeit, wohin ith zu

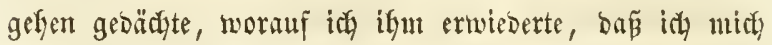

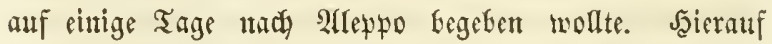
fagte or nidgts, aber inbem er in meinem (5ietärá zmei meife Deffen bemerfte, bie id von Marjęlle mitgebradyt Gatte, erjudgte er midh, biefelben zu verfaufen. Sid fot

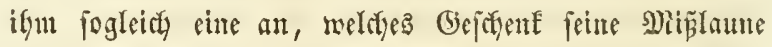
milloerte.

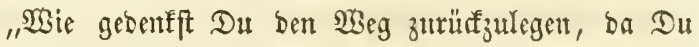
feim Pfero befibeft?" fagte er zu mir.

"Deine Benterfung ift ganz ridftig," antwortete id)

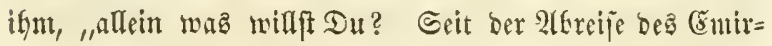
2(kbor (Stallmeifter von ßortes) bat man mir nicht ein cinzigez Pfero getract)t, das idy Gätte faupen Ënuen."

"Das ift, weil Du Didy z" jamer befriedigen läfieft."

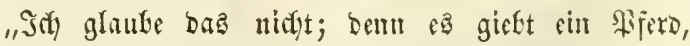
weldyes mir jein Eigenthümer nidyt verfaufen mollte, of = gleidy idy it)m zu zalylen betwilaigte, mas er gefordertt batte." "llno melcoges ift bas?" 


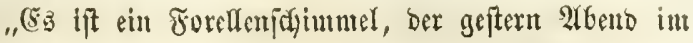
Etammte anfunt."

"(5i, füflidy)! 2lber Du forberft zu viel. Theist

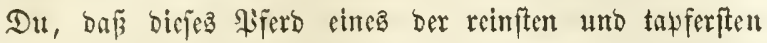

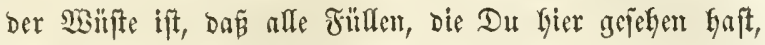
jeine Sintor find und baß́ ber Mléléan, welcten ber Emir = 2lfyor mit fid) genonmen Gat, gleidffalla jein Solyn ift?"

Sebcs \$3ort, bas Dufaï iprad), ftrigerte un meine

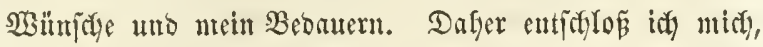
Den Lebeten Berjud zoll wagent.

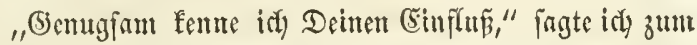
Sdyeif, „um überzeugt zu jein, Daß́, wollteft Du nur ciut

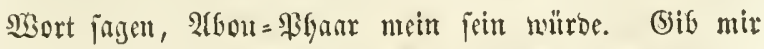
aljo cine Jreundidyaftaprobe in biejer Angelegenbeit und jei mir befülflidy, ben Jandel anzufnüpfen; beendige id ifn, fo werbe idi Dir banfbar fein, inbem id Dir 50 Siafter (37 Jranfen 50 (Eentimes), aโక Bacţis gebe."

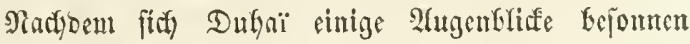

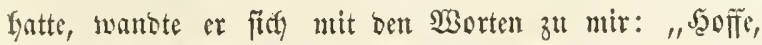

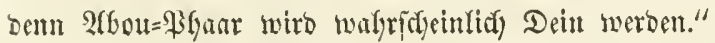

Sogleidf) rief er einen Araber, Gefafil ifm, ein Dro=

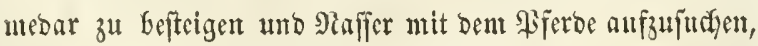
welder fich mit bentelfen ungefäfr zwei Stunden wou

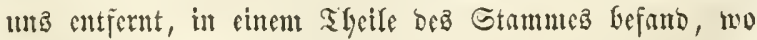
2rfou= Shant mefre Etmten zu Geberfen batte. Dem

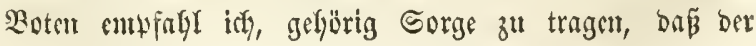




\section{0}

MGon=Phaar nidjt etma ofne Sattel uno Bamm anfäme; worauf er fith entjernte.

Şäfyrent bieję vorging, hatte fich bie Rarawane

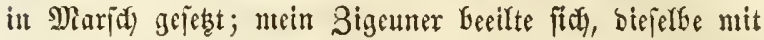
jeinem Siameele unt aflen meinen Sagajeligfeiten einzulgolen, mto idf blteb mit Georg unt Gaid zurüaf. গoth barf

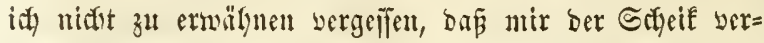
fiprodjen Gatte, mir bie Mittel zu verfdafien, bie Tur=

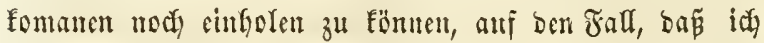

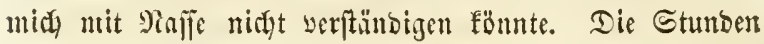
werftriçent; unb bie llugeduro, mit meldyer id ben Boten zurüf ermartete, ift wolgl reingt zu begreifer. Defto gröbir mar aber baum meine Freube, als id 9iaffer in einem ßeiter wieber erfannte, welder mit einer reizenden Edynelligteit des Stammtfyeiles auf umb zufam, won wo

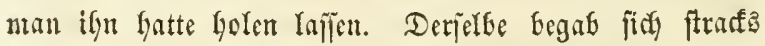
in bas Belt bes Dulyai, wofin wir ifm folgten. Snoen

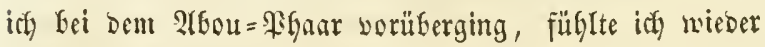
alfe meine Befürdftungen in mir ermadyen; unb obgleich) berferlbe ofune 3aum war, und fein ganzer Schumut nur it eiutent ber forlidfteften Sättel Geftamb, war er mir bod

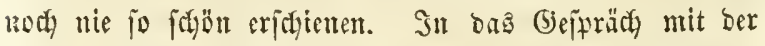
Frage eingefento, weldyen Grumb Siaffer habe, feim Píero sque 3aum zu reiten, antmortete or mir: "Wrotl er feimen utöthig hat." Dufgä nałm ifyu hierauf bet Seitc,

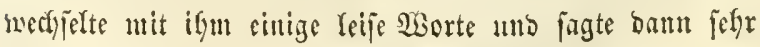

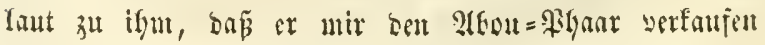




\section{1}

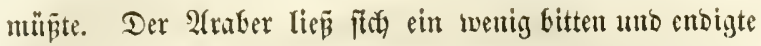
bamit, midy einzullaben, ilgm meine Bjebote zu tyun. Diejes liés idy mir nidyt zmeimal jagen.

"Geptern 2lbend," jagte idfy, "bot idi Dir 2,500 Piafter, feute lege idy uod) 100 bazu.

"Das ift nod nidat gemug," antmortete Nafifer, "Giete meiter."

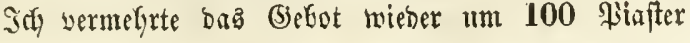

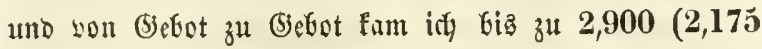
Franfent). Sor war entidaldofien, noch weiter zu Gietert, alz ber 2 trater, auf mitch zufommento, mir enblich bie

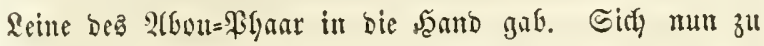

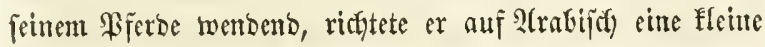
2turebe an bajjelle, worin er jagte, bã jie firs tremen

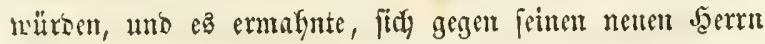
afs eben io geforjamen und treuen Dienter zu Eeweijen, mie Niuto barauf nit TaGafraud anfüllend, näferte er firty yon neuem feinem alten befülgrten unto Elice ifym benjerts

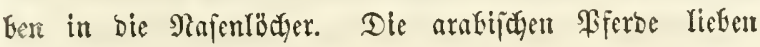

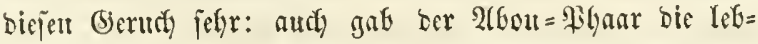
haftefte Frembe zu erfenmen, erwies feinem Şerrn eine Mienge yon Ricbfojungen, bie yon Resterem neue Freundo=

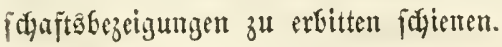




\section{2}

\section{Sicbentes Siapitel.}

Zugang von. Tel =el = Sultani, - Sorfälle unb Eefabren in ter

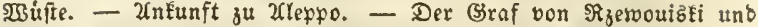

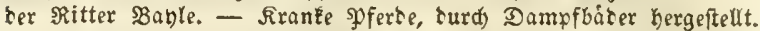
- 2nfunft einer nad̆ Mefka gehenten perfifden Rarawane. Ihte Spferte. - Solle; sie 2 rt, wie tiejelben eingefortert wertent. - Dubaï uns Daus in 2teppo. - Bertoeigerter 3roeifampf. Serr Gontolwhy, SBifdof vom Ribanon; fein Einjug in sie Stast

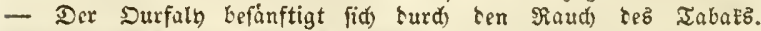
- Jbreife nad Saira (Seiteh ober Sait; eberem Siton).

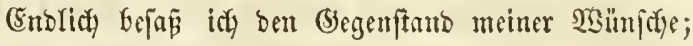
alfein ich befand mich unglïflicterneife in ber Sisüfte,

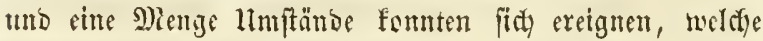
mir mein netes (Eigentfyum jtreitig ntadjer. So war aud mein beld mit ber Saramane afgegangen, und wat mir alo unmöglidy, die 2,900 \$iafter, ben Fireis

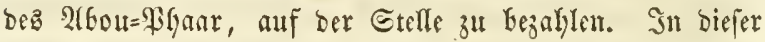

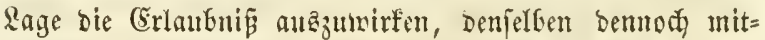
zutuhmen, war feime loidfte Enct)e, vorzügYidy ment man

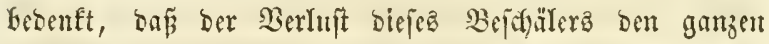
Stamm in Unrulye verjetzen fonnte. Sith vertraute midy Dem Georg; allein weit entfernt, meine Bejorgnifife zu

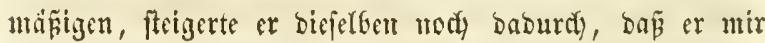
jagte: bie 2raber füfrten (Etmaz gegen mid̆ tm

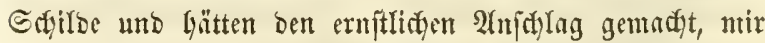
Den 2lfou= PGgar zut entreißen. Sd verlor mid barauf im Nadjbenfen über bie Mittel, mid auz biejer $\mathfrak{B e r l e s}$

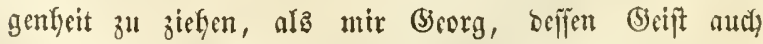
utidyt untfätig gefliefon war, ein Piettungsmittel angab. 


\section{3}

"Befteigen Sie ben 2(Gou=\$Gaar," fagte er zu mir, "als wenn Sic ifjn probiren wollten; wenn Sie bam it einiger (Entfernung vom Lager fitto, fo begeten Sie

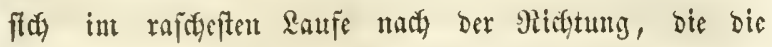
Saramane cingeifalagen hat; haten Eie bieje crrcidyt, fo

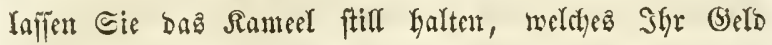

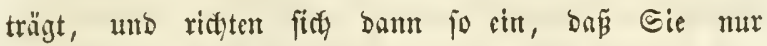
bic verabrcoete Enmme zu bezafylen haben, memt idf bei Styen mit ben Bebuinen anfomme, bie midh fut SGrer Berfolgung mit jitfy netymen meroent."

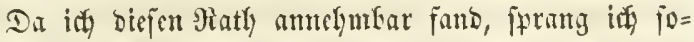

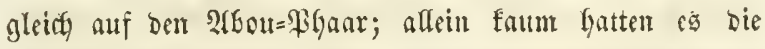

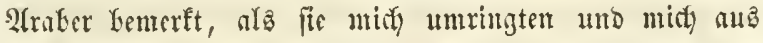

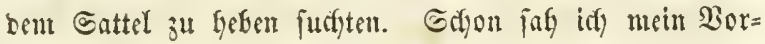
Gaben int Seinte erfitift, als mir ber (b)eoanffe cinfan,

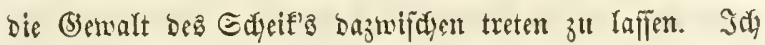

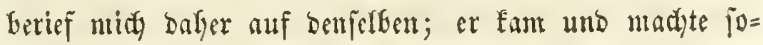

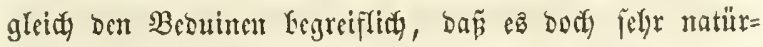

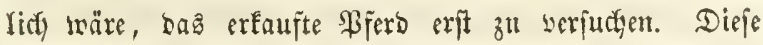

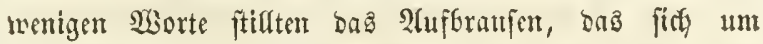

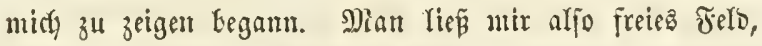

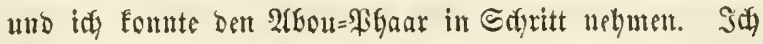
glaute gejagt zul haben, oás or obne Baum war; ida

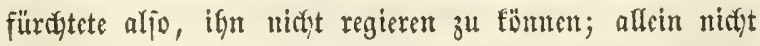
Iange Beit hatte id nötgig, un midy won bem gut über=

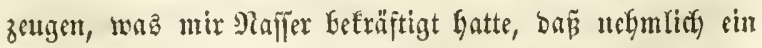

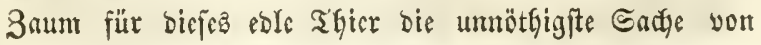




\section{4}

ber Welt wăre; benn faum war idy auf Flintenjŭup̧weite sont Rager entfernt, ala id nur bie Steigbügel ben Flanfent zu näfern braudyte, um jogleiry un midy her Staubwirbel zu erregen, jowie mid, nad) eintigen Gefunden ben Blifen meinter guten Beduinen zu entgiefen, unb in weniger benn einer Stunde Gatte id bie Raramane eingeholt. Innvergüglia

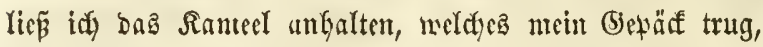

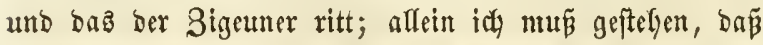
idy tur einen fleimen Theil ber Sdyroierigfeiten vorausz gejeben Gatte, melcte mid ermarteten. Der übrige Theil oer Truppe Gatte feinen Diarid feinesิmegs aufgehalten; mein Rameel molle baher nicht zurïfbleiben, web̆galb id) genöthigt murbe, dajfelfe, um verfindern, nieberlegen zu lafjen und ifnt bie beiben Bor

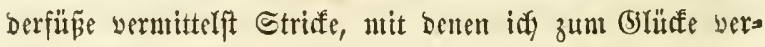
feben war, an bie Borarme zu Ginden. Dieje Borfithtes=

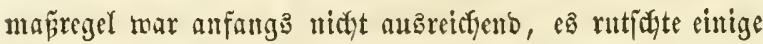
Seit auf ben Suteen meiter unb berubigte fid erjt bann, als

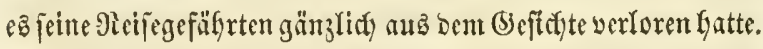

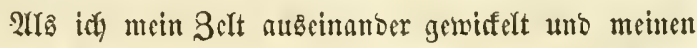
Diantelface geöfinet batte, fing idu anf metmem Mecdylaz (arabijaden Miantel) mein nod übriges (beld zu zäflen

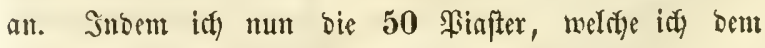
Dufai als Bachis verjprodyen hatte, nit bazu redjnete, hatte i出 2,950 (2,212 Jranfen 50 (Eentiutes) nötGig; id) modyte aber zäflen, wie idy mollte, fo fornte idu uidat mefye als 2,100 Piufter zulammenbringen. 


\section{5}

V̧̧ medte ungefäly cine halfe Stumbe vergangen

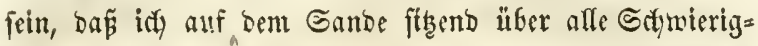
feiten, weldfe nteine fonberbare Rage mit fith bracte,

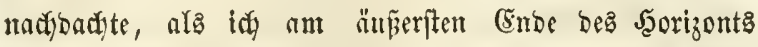
einige $\mathfrak{A}$ raber erblinfte, bie, auf wortrefrlidben Stuten fibend,

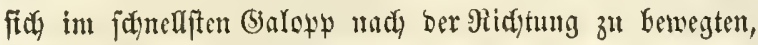

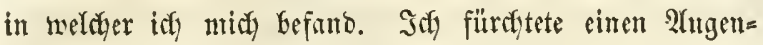

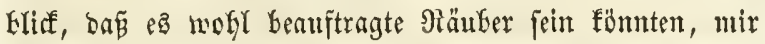

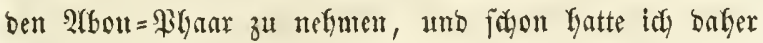

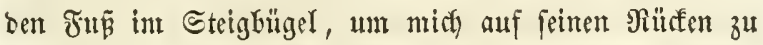

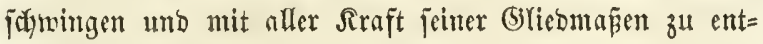
flieber, als idy Georg hinter Saib auf cinem fraftuollen turfonanijacn Pferbe ftizen jah, bas die Spize ber

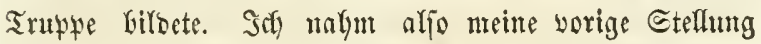
mieber ein unb wartete. 2Is fie bei mir angefonmen

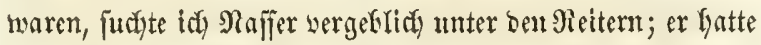
aber nidyt gewagt, fie zul begleiten, unb war burds Daub ericht morben. Sof jagte num biefem Sebteren, bar ich feinçmegs die ganze mir nöthige Summe $\mathfrak{b} c=$ fäpe, un meine Schulo abzutragen, und baß̃ er Durdjaus

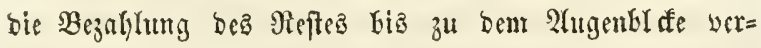

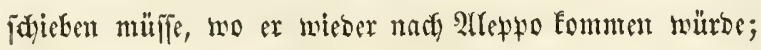

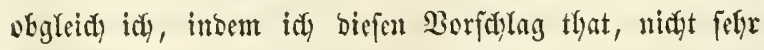

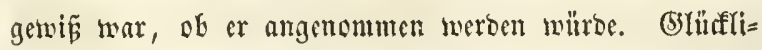
(berneife zog midf Said aub ber Berlegenlyeit, inbent er mir unverzüglith die mir feblenden 850 Piafter vor=

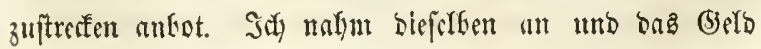




\section{6}

murbe bem Daus aufgezäglt, ber mir barauf fagt,. Dá er 50 Pinfter als Brrtyis für bie Mühs, fta fierfer zu begeben, verlange. Sidy madite mith igm bieje zu bes zablen verbindich, wenn er nach $\mathfrak{A}$ (leppo fommen würbe; frood unter ber Bebingung, bafi ar mir bie nad meiner 2lntunft in Rager geftoblene Lauze zuftellte. (Er wer= (prad) nir bas, unb fügte Gingu, bap̃, ba id nady Slleppo

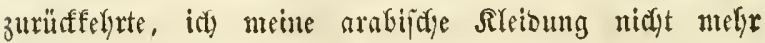
nötyig bätte tmb er miç baher bäte, ifm biẹelfe zu geben. S(4) madyte ifm barauf bemerflidy, ban idy nicht nafft gelyen fömnte. Statt aller 2lntwort zeigte or auf cinige feiner (Sefäbrten, beren einjige Sleibung in eimem Stïfe wollenen Beriges beptant, welctes fie über ben Şütten befeftigt trugen. SeG zeigte aber gegen biejez

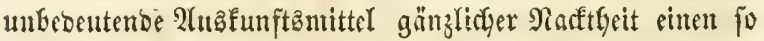

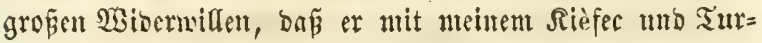
ban worlicb nelymen mollte. Jad jagte ifym won neuem, bัธ

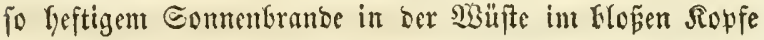
zu fein. Sn bemfelben 2 agenblide hatte idf gerabe ein

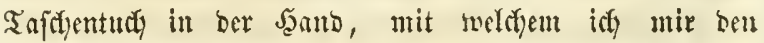

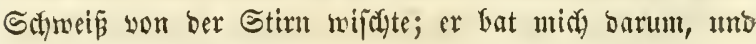

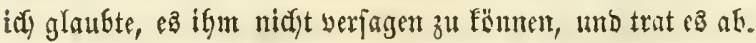

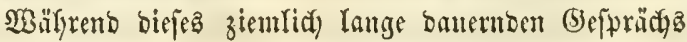
Gatte Beorg unb ber Bigeuner all' mein (bictärf mieber auf mein Sameel gelaben; Frioc Gatten es baranf beptie= gen unb saffelfe in Marja gejebt, um bie Siramane 


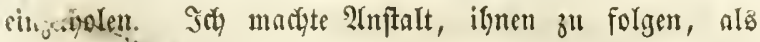

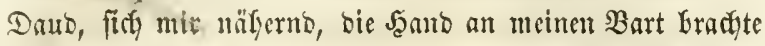
unb ifn fïpte. Die andern $\mathfrak{A}$ rafaer madjen mir eine tieje $\mathfrak{B}$ ertetrgung, bie mit bem $\Re$ hefe: Allah yahh fedak

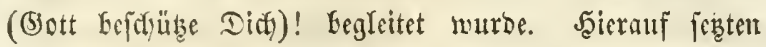
fid alle, fo wie and Enib, auf iffe Etutert; id fprang

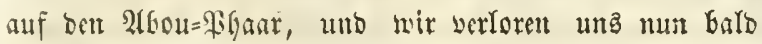
aus bem (Seficidete.

Эd) ritt tufig eine halbe Stunbe im Sdyritt, als id

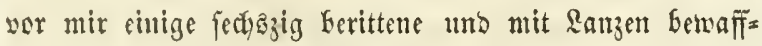

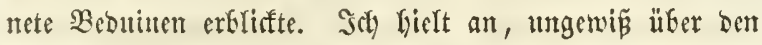

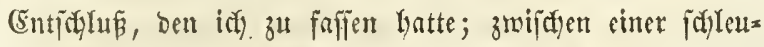
nigen Fludyt nach ber sildytung hin, wo id efen berge= fommest war, und einent werwegenen Durdjertd durch bie Truppe, welche mir ben $\mathfrak{S e g}$ veriperrte, fyatte idi zu

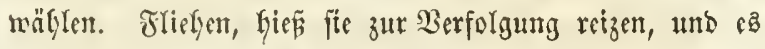

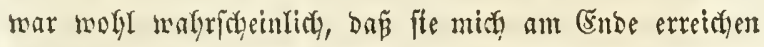
muẼten, beme ifge Stutest fonnten noch frifder und folg=

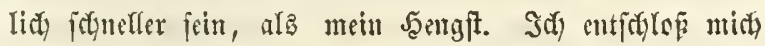

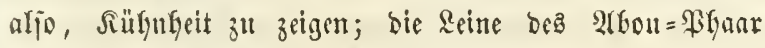

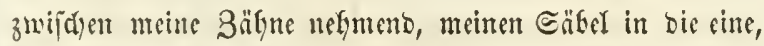
cin Siffol in bie anbere Saand, fwrengte idf mein Fifero

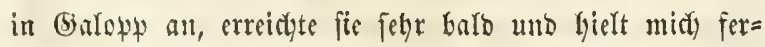

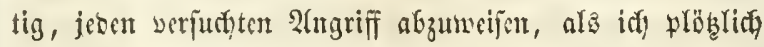
alle Ranzen untefren und bie Epizen in bie Crotoe fteffen fah. Nein Errftaunen war großß, aber furz, Denn indem

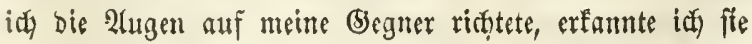




\section{8}

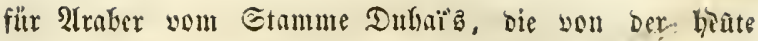
Morgen abgegangenen Raramane zurüffamen, beren $B_{\ell}$ gleiter fie marm, unto weldac, mith fefend, geglaubt batten,

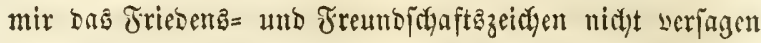

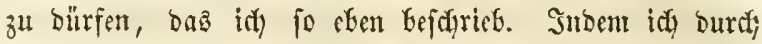
fie ritt, hörte idf Den Miff: Mach-Allah, achim-bachi, ada

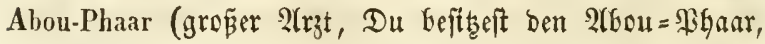

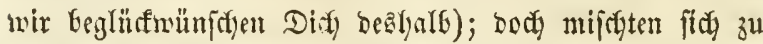

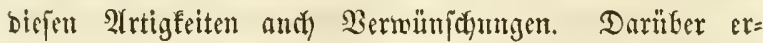

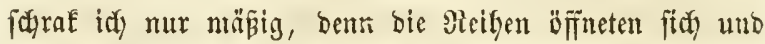

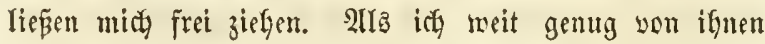
entfernt war, nafm idf ben 2(Fon= PGaar nicber in Sedritt, um ign für eine neue Belegenbeit zu fodsonen; mo idi) Yatte allen (Srumb, midy biefer Sorfictst megen zu loben, bem balo görte ith nod ziemlida weit binter

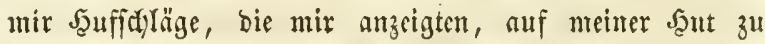

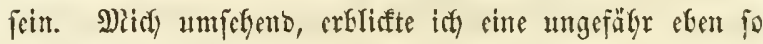
beträdytliţe Sctaar Bebninen, als biefe mar, meldfye idt

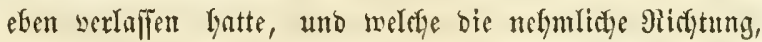
wie id, zu verfolgen fdiemen. Die Beobardtung you

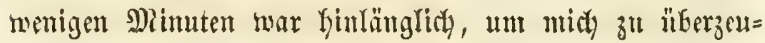
gen, baj fie midy werfolgten; benn bald liejen fie ifre Siferbe im ftärften Galopp fortictefen, Galo magmen fie fte wieber in Sdyritt, wic es ligre Semofnbeit ift, fei ç

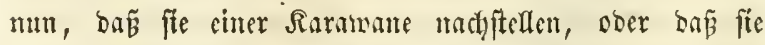
fliefyen. Эa) ridytete bie Sangarten meines \$ferbes nad

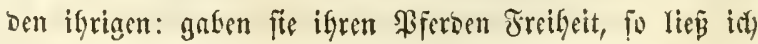




\section{9}

Das' nesinige laujen; hielten fie bie ifyrigen auf, fo that

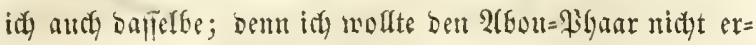

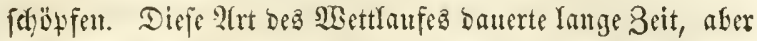

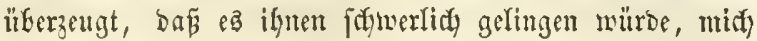

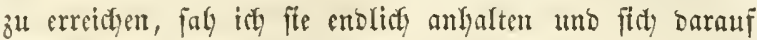
entfernen. (5ott weís

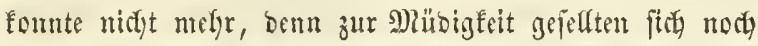

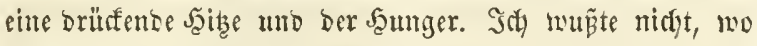
idi anfyalten follte, als ide endich an bem llfer eines $\mathfrak{B}_{\mathfrak{a}}=$ ()

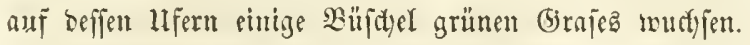

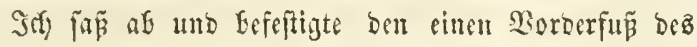

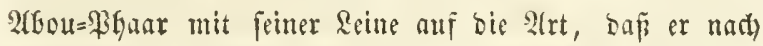
(befallen meiben fonnte, mälgrento idy an bie Duelle ging, um mir ben Bart zu majofen umb ben Durje zu löjofen. 2(n berjelgen angefonmen, fand id bajelfopt brei Bebuinen

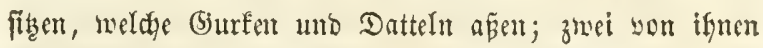
waren mit fouledften Säbeln und ber eine mit einer $\mathfrak{R} u n=$ tenfritute bemafinnet. Eobalo fie mid faben, gaben fie ein

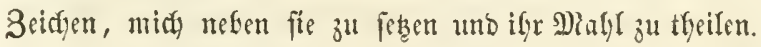
Da mir biefe Cinladumg zu crmünifgt fant, mut fie zmet= nal wicberlyolen zu Yafien, nafyu idy Plat. Saum gatte

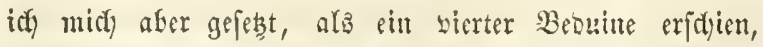

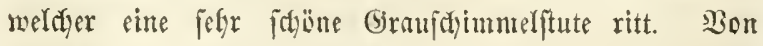
feinem Sijerbe abfeigen, fidy, olyme gebeten zu fein, zu

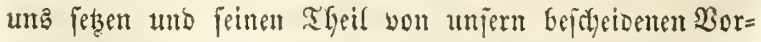
rätfgen nefmen, ohme bá ifm meime Geber baju nütfigten, 


\section{0}

war für ifn bie Gadye mentiger Sefuntert. Miads been= bigter Maflygeit ridfteten fitd bie Hugen bes Neunge= fommenent mad) ber Seite, wo fid ber $2(b o u=$ Phaar be= fanto, weldaer in bicjem 2hagenblife bie Etute witterte

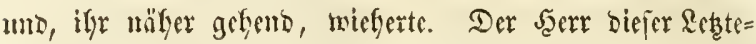

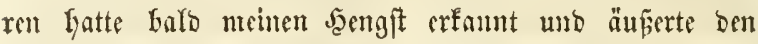

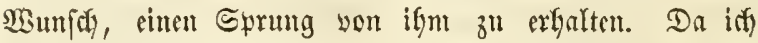
aber nidyt wiffell founte, of mir nidyt nody andere lin=

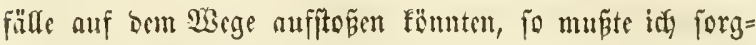

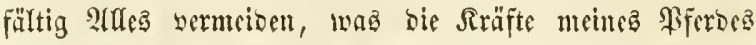

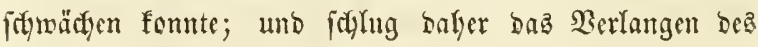
Trabers rund ab. Diejer fojiea barüber beftig erjürnt

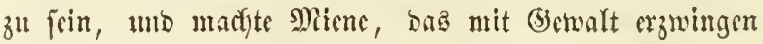

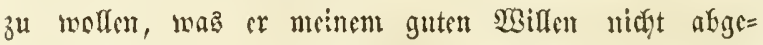
winnen fonte. Schon war ar anfgeftanden mo batte

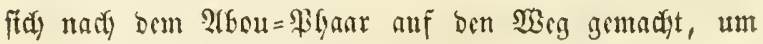

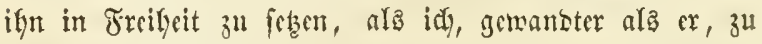
bom Restern Yief, bie Reine, weldye igm ben Jü zurürf= bielt, roşríp und ntidy in ben Sattel warf. 2(lz ber Slaaber midy zum Flieflen bercit jah, furang er auf feine

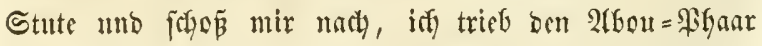
an, und mägrend einer halben Etumbe hörte mein Geg= ner nidjt anf, midr zu werfolgen. Sthein mein Sifero

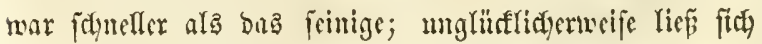

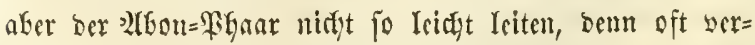
Gielt ar fich und mollte zur Stute. Diefe sage murbe

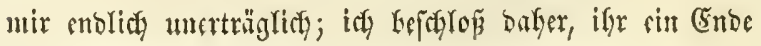




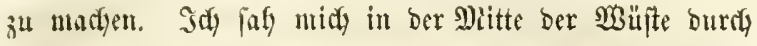
cinen einzigen Bebuinen verfolgt, weldyer mur eine $\mathfrak{L a n z}_{\text {and }}$

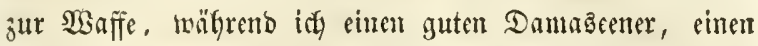
Sarrbjax (zmeifanneibigen Dolda) uno zwei Siftolen in

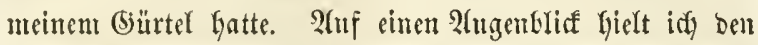
Gebanten feft, ben 2 araber zut befämpfen und ifgm feime Gtute zu nelgmen; allein idf murbe burdy bie Befürdytung zurülifgelyalten, fräter anberen Bebuinen zu begeguen, bie, meine (5roberung erfemment, mir biejelte unfegltar, nact)= bem fie Bhut mit Blut bezahylt gemadft, wieser entriffen Gaben mürben. Să fannte bie religiöfe Geswiffengaftig=

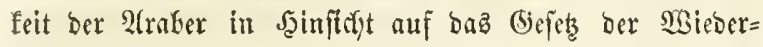

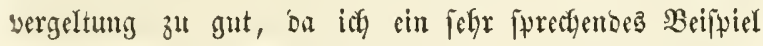

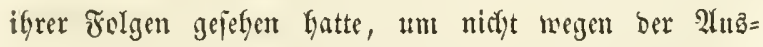

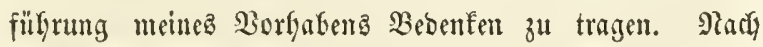
einiger Heferlegung vergitutete id barauf uno begnügte

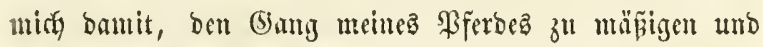
meinem Begner brofenbe B̧iffe zuzuwerfen, welcher, ohme Sweifel burch meine Gebersen und ben verältberten Bang

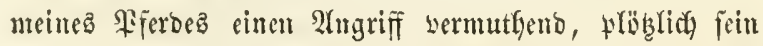

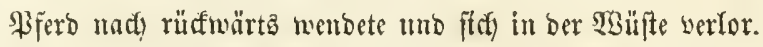

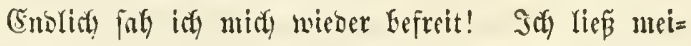

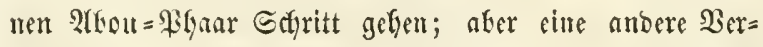

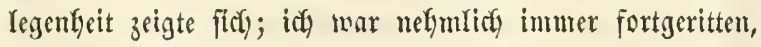
ofne in geringften zu wifien, mofin idg midy begab; benn bie Wrälite befibt feine abgeftecften Tresge, faum

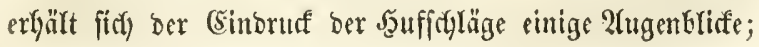




\section{2}

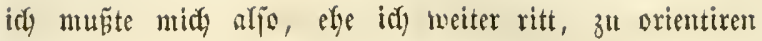

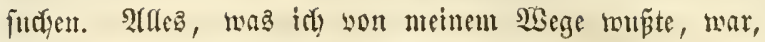

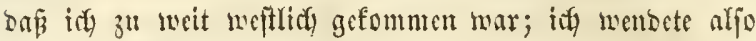

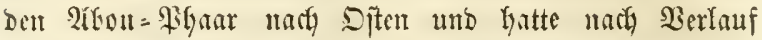

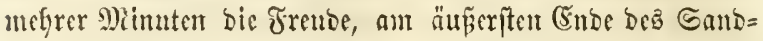
Gorizontes, ber midi untgab, bie Spiben ber Minareta son 2llepto zul gemalyren. Der Marjes einer Ctunde reidyte fyit, midy ber Etast fo weit ju täfern, un bie

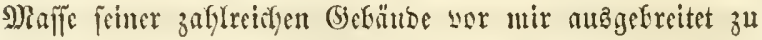

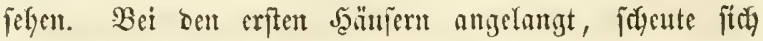

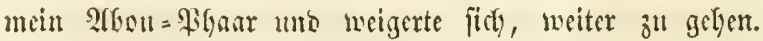

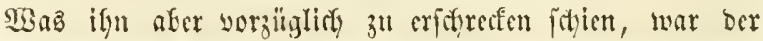

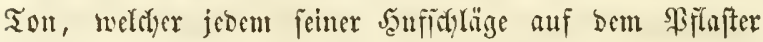
folgte; er fenfte jebesimal ben Sopf und bejafy den Boben

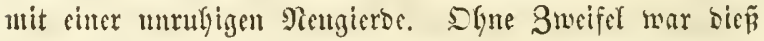

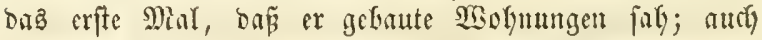

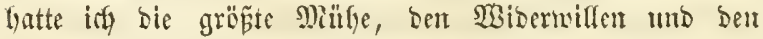

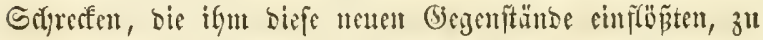

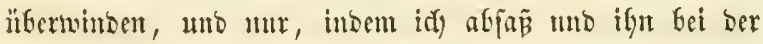

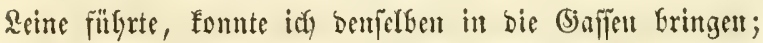

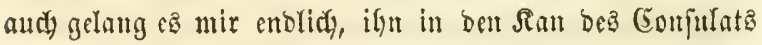

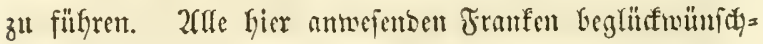
ten midy iregen meiner Sisiescranfunft um fo lebthafter, ba

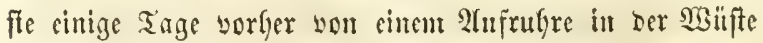

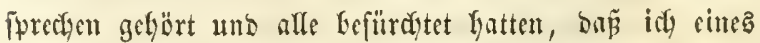
ber eriten Dyfer befieleen gewejen jein mürbe. 2lla die crften, gänzlic ber frenbigen Theiluahme gemiometen 


\section{3}

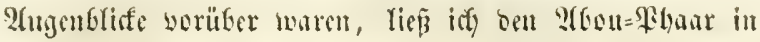
sen Etall ziefen Int cilte bant in bnz Bab, um bort gegen curctäiffe fleisung bas won llugejiefer ftrobente Coftü zu wedyfeln, bas id) feit meincm (Entritt in bie

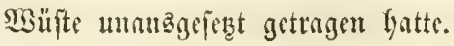

Die shadfridyt won meiner Anfunft verbreitete fith felyr Falo in ber ganzen Strot. Der grö̈te Theil ber Franfen, bic ffet) bort aufgiclten, fauten, micts ju befuct)en, jowie zu gleider Beit ben 2fbou=\$3gaar zu fefen. Ilnter

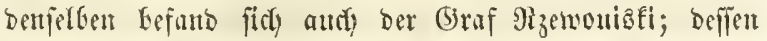

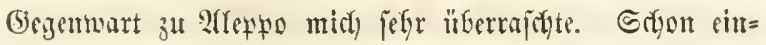

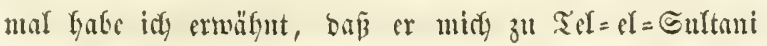

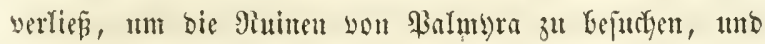
fich wou ba zu mefren arabijexen Stïnmen zu begeben,

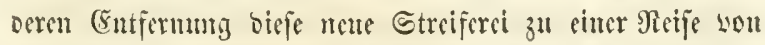
lauger Daner gemudyt baben witrob. Sơ änperte ifyu

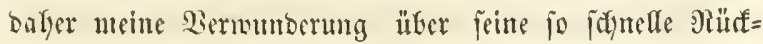

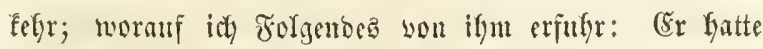
wenige Tage möthig gebaft, un wom Rager Deß Dubai

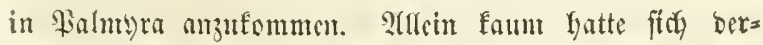

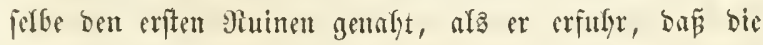
ifum zur Bebefung biencmben Bebumen Böpes gegen iqn

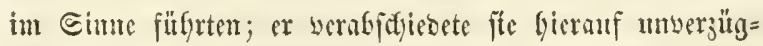
(ic) füumtlicf) uno ging mit neuen Füfrern, nus Ctaume, Der bei ben berïguten Ileberreften lagert, unb

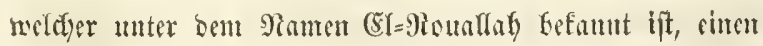
Yccoro ein. Bon neuen unterridytet, bañ ber Scheif biefer 


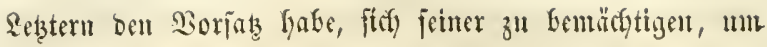

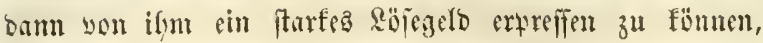

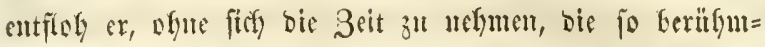

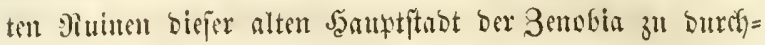

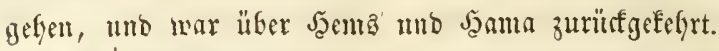

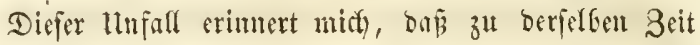
sin englijotyer Reijember, ber Mitter Bayle, nach Matepwo

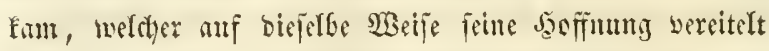
faly umb beflagte. Diefer hatte efenfalls breipig Bebui= nen aแล cinem ber Gtänme ber Foebanż=2(nazés genom=

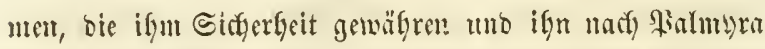
geteiten follten. Heberbies war er von cinent 2(recyiteften und zmei Sagarfitmeiftery aus ber after faiferlicfen Garbe begleitet. Estion war er nahe am Biele feiner Rimitreife, alz $\mathrm{er}$, fitch mit bem STrchiteften unterfyaltento, Dem Mefte ber Trupte un cinige Eafyitte vorangefommen war unb

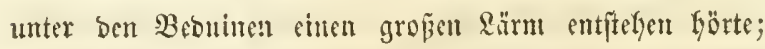

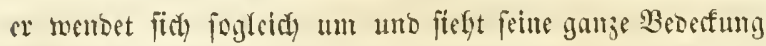
iun Sandogemenge mit ben beiben lluterofïzieren. Thugen=

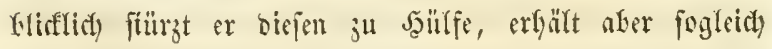

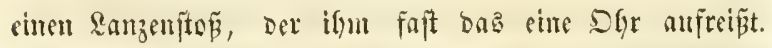
Diefe Bentllfomumung femog igh, eime fichulle und

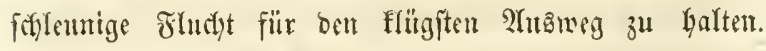
Die 2 and tmeifter waren aber hartnitfiger; benn fie moll=

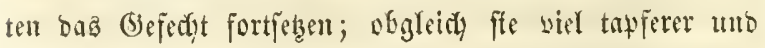
weit gefdyifter, als iffe beguer warell, fo bebrobete fie

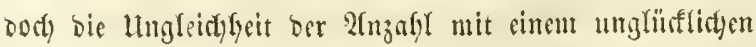




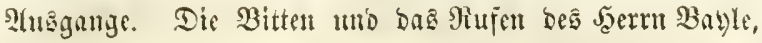

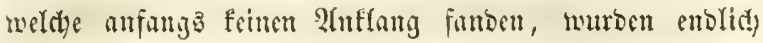
yon benjelfon gefiört; fie folgten ifum unb alle vier nafmen bie glicftung nach Sarma. Jgre Bebeffung verfolgte fie Iange und gab exft bam ifre Beute auj, als bie

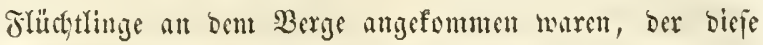
Stabt beherridt. Saum war ber 9litter $\mathfrak{B}$ ayle in Sactu

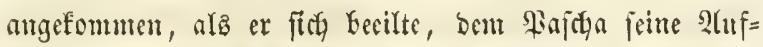

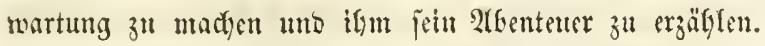
Der ottomanifice SGüroenträger verfor feite Beit tmo

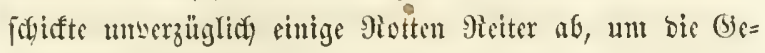
geno zu Durdyftreifen; bieje fanten ben 2 f6ento nit mefren auf ifre Ranzmpizizen geftecten Röpfen in bie Staht zu= rït. 56 mun bieje menjdylidyen lleberrejte Reuten von ber Bebectung ober einigen unglïflidyen ganz frieblidyen

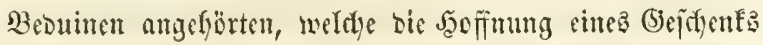
yon einigen Pinftern, das Der Bejţ biejer angeblidyen

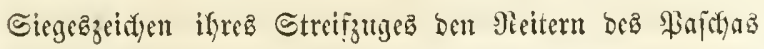
cinbringen follte, mit ben Reben bezablen musten, das

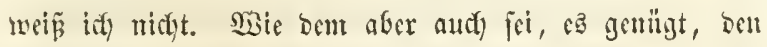

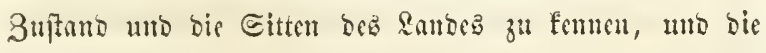
(sintfermung zu bebenfen, in weldyer fie fidt) zmifitgen der

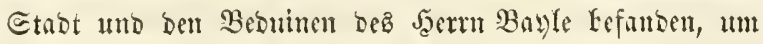

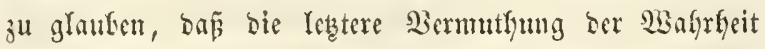
am näd)fiten liege.

Sot) fefre zu Serrn von sizemontaft zurïf. $\Xi_{0}=$ balo biejer ben 2lbousßghar angejegen hatte, rief er aus: 


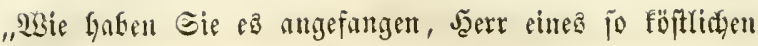
Tifieres zu weroen? Nie traf idf ein \$iferb an, bos mit

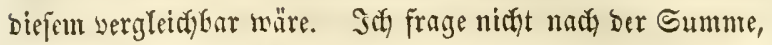
bie Eie Dafür gegefen bafen, aber beftumen Sie mir

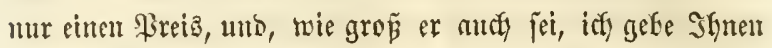
benfelten."

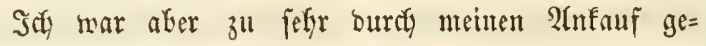

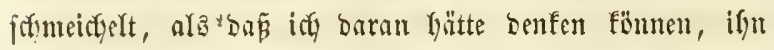

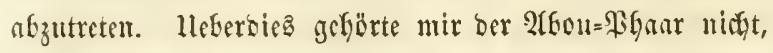

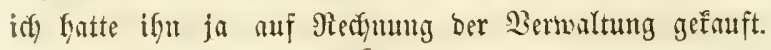

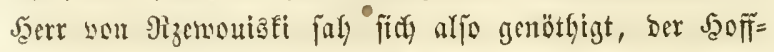

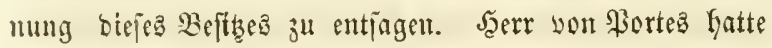

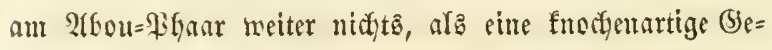

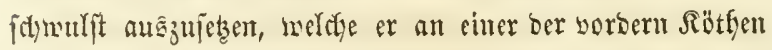
Yhattc; fie war aber nidft gefäfrlict, weil fie nur bie Folge eines äfers bei ben arafifden \$ferben vorfoumen=

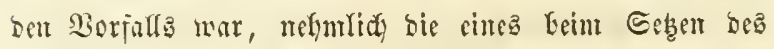

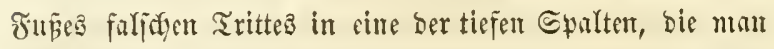

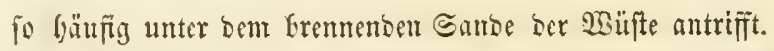
(rrft am 3meiten Tage und meiner 2tufumft fam Grorg in ben Mauern yon 2 fleppo mit ber Rameelfara= mane an, weldye zu Derferleen Seit mit mir bas $\mathfrak{a} a g e r$ werlajen hattr. Da mir niçt bie Beit gegännt mar, mid) mit ifm zu unterbalter, ars er midy mit Damo

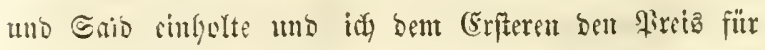

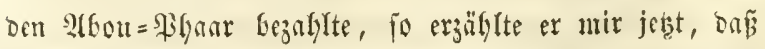
fict) bie sosuinen friner fogleidy nad) meinem plöglidyen 


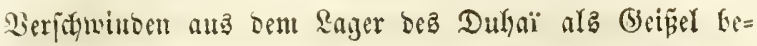

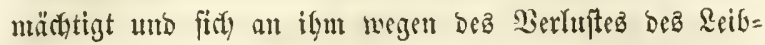

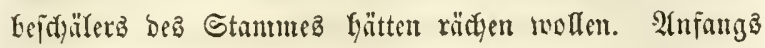
gätten fie bavon gejproden, ign in baв Gefängníz zu

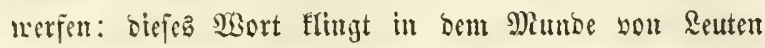
ganz fonberbar, welefe feime andern Mauern feunen, als

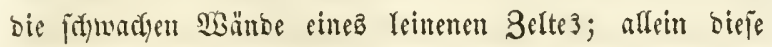

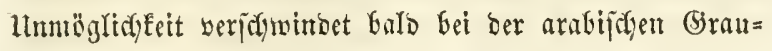

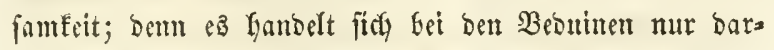
um, in ben Ganb ein zienlidy tiefez 20 dy zu graben, fo bấ won bem Gefangenen, ben man binein geftellt rat, mur ber Sopf \#̈ber bem Eroreidy bleift; barauf filllem fie bieje 2rt yon Grube nieber mit bem Sambe zu, ben fie herauggenommen baben, uno Ia fen ben Berurtbeilten

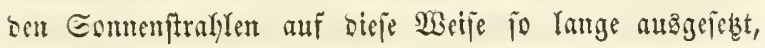
alอ

(5eorg war bariber nicht menig eridfrocfen; allein peine beifteagegenwart bald wieber fammelno, nuafite or

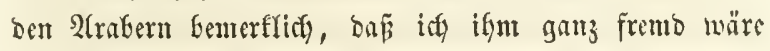

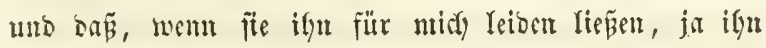
felbit bem Tobe opfern nouten, fie defiwegen nicht ben

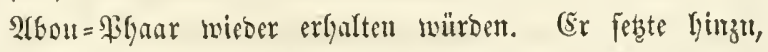
Dá̃ zu ftelylen, unto baj er berfidjert wäre, id bätte mid nux entfernt, um ber Sarawane nadjzuteilen uto baz Gelo zu loolen, meldes flit) auf bem samedle be=

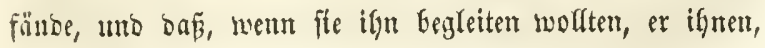


in bem Falfe, wenn er ffe betrogen Gätte, werīpräđje, alle Qualen z"l Yeiben, weldye bie Rügen unt bie Faljoblyeit verbienter. Dieje 2(euferungen fofienen auf bie Besuinen (Einbruff zu madyen; benn madyoem fite unter ftedy berath)=

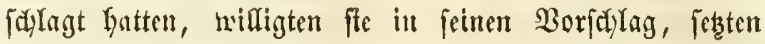
irgu rinter Gaib auf bas Sffero, wie idy oben erzăhlte,

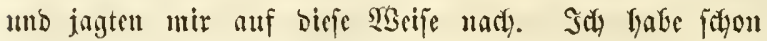

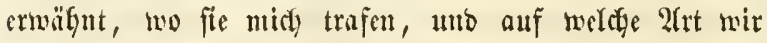
unz tremuten.

ŞGenige Tage nad) nteiner Burildfunft aub ber

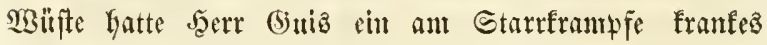
Siferb; idf wenbete balyer, um bemjetben Rinderung zu veriffarien, affe Mittel an, bie mir bie Runit zu bieten

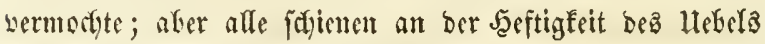

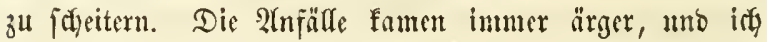
fing an, bie bjemejung zu bezmeifall, als ç mir einfiel, bem Siranfen Dampföber zu geten. Die Seftrierigfeit babei lag barim, fite ifgu beizubringen. Saerr Eamifin v.

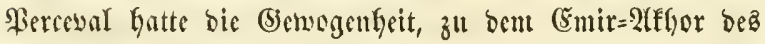

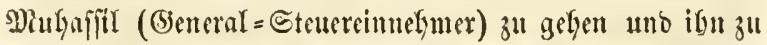
Gitter, ung in bie bifentlicten Bäber zu begleiten unb ben

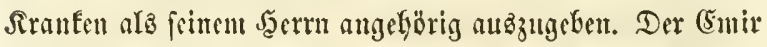
milligte gefälligft cin; als wir una aber fyicrauf zeigten,

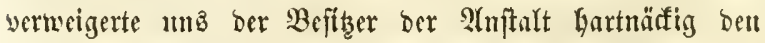

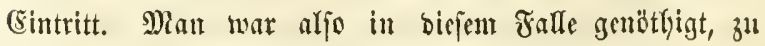

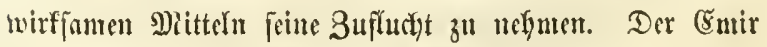

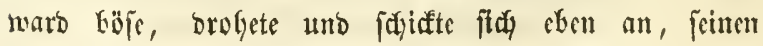




\section{9}

Bermünfđungen ben überwiegenoen Brumb einer ftarfen Tractyt von Stodicțlägen beizufïgen. Diẹe 2lrt, zu über= jeugen, bejamitftigte allen $\mathfrak{W}$ iberwillen und alle $\mathfrak{B e b e n t}=$ lichleit bes ftörrigen Türfen; er beeilte fidy, alle fitu fier Gabenben Diujeluämer ju entfernen, unb geftattete nno enolich, ben Sranten in bic Säle zu füfren; wofeleft biejer bem ftärfften Dampfe lange Beit augegejetgt bricb.

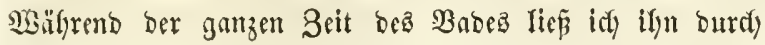
Reitfuedfte, bie idy nitgenonmen Gatte, abreiben, uno

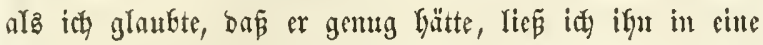
meniger erftixento beipe artetyeilung bringen, wo man benjelben in fefr warme Deffen hitllte, mb erfaufte ifgti

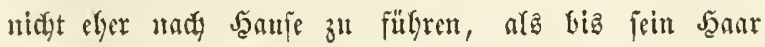
günzlidy trecécu war. Diejea erfte Bab Gatte den beften

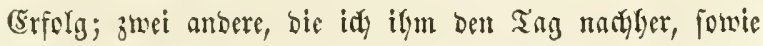

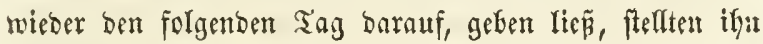
wolfommen Ger.

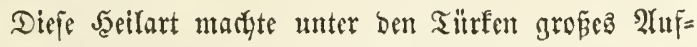
fefen; alle riefen whunber, unb yon biefent Tage an murbe das $\mathfrak{B a d}$ ein affgemeines Seilmittel fïr jebes Rei=

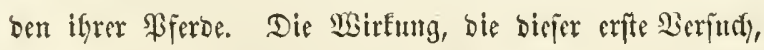

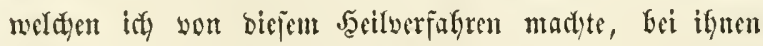
Geroorbradte, war fo tief, bas fie midy nody geute, menn

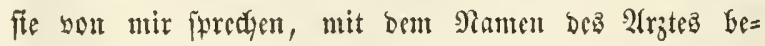
zeidynen, weldyer ben SFferben Bäber gab.

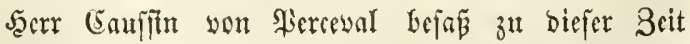

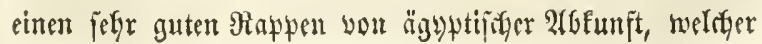




\section{0}

Weftige Solif befommen Gatte; Den leţten berartigen $211=$

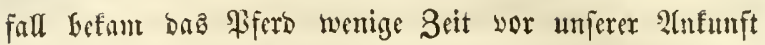

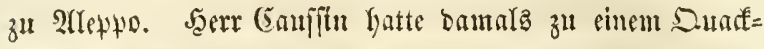

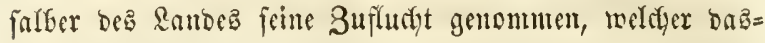
jenige Satilungåmitte! in 2 Inmentoung bringen mollte, befifen

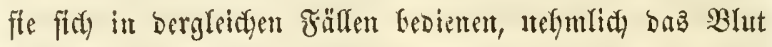
eines jungen Şumbes, ben fite errü̈rgen, und das fie bem Rranfen warn cingeben. Dicjer Maun latte einen armen

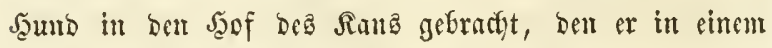

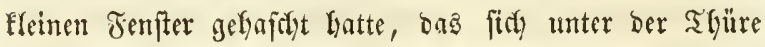

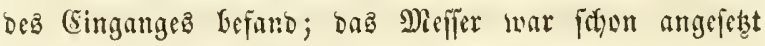
und baв Dpfer follte cbert fallen, ars der Dyferpriefter

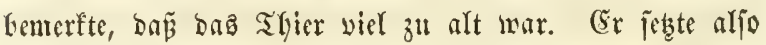

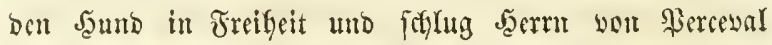

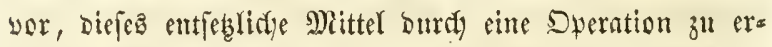

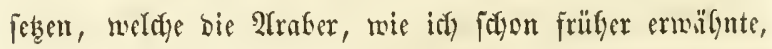

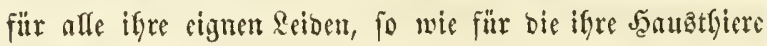

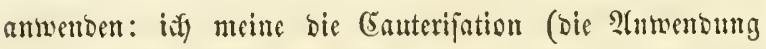
bę glüfenden (Eijemb). Seerr von Perceval, der um jeben Preis fein Pfero resten mollte, willigte ontyer cirt. Der Sunaffarter zündete foglecid ein grofies fenter an und

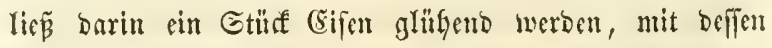

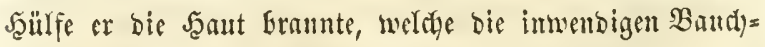

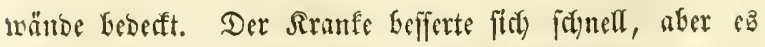

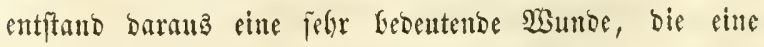

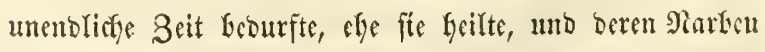
bas Thier biz zu feitem Tode belyielt. Da bieje Rolif 


\section{1}

alle Beidyen einer Geftigen Darmentzünomng batte, fo liéz

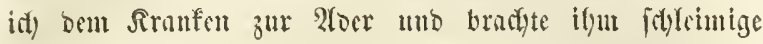
Mittel, thile als Getrün, theila burdy Sliftiete bei. Da icf) aber fah, dap bie Symprome biefen Mitteln nicht weidgen wollen, fo matym idy mir gleidyfalls vor, bic

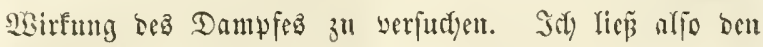

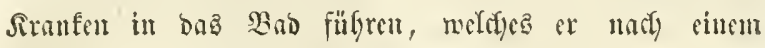
zoreiftumbigen 2lufenthalte volffomman genejen verlief. 3war fatte ich bie grögte Sillfe, ilgn fier rubig fil er= Yyalten, ba er fo jefre aufgereizt war, baf ex fite faum anf Den fefre glatten Etcimplatten, weldye ben Boben beB Smucrn bicjer Afuftalten bilocn, zu erfyalten vermodte.

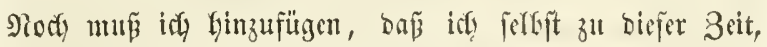
an einer Gebeutenden Ruftröhrententzäntong litt, welche woll= fonmen burdy bie blope Premejenlgeit in bicfen Bäbern weridgmand. Soly gebe nicht weiter, ofue noch linzuzufïgen,

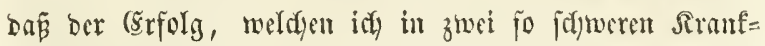
'Geiten, wie bieje waren, you benten ich cben gefprodien Gabe, yout Dampfe erlangt Yatte, mid in Franfreida anf ben (bebanfen fratate, beffen 2hmentung anth anf andere

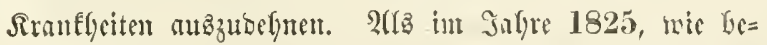
fannt, eine verberblidye Riebfeutbe einen grofien Theil unjerer Devartentents mo sorzüglich łaris werbecrte, fo

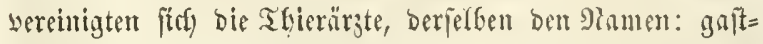
rijije Darmentz̈mbung (gastro-entérite) Geizulegen. S(t)

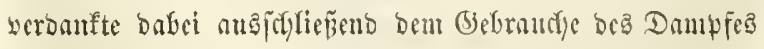

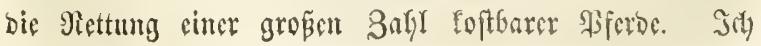




\section{2}

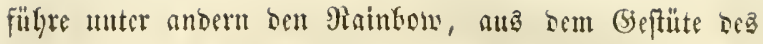

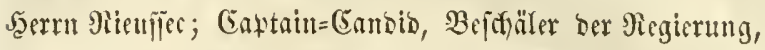
und melye antere Pferbe Der föniglictum Depets zu Mabrio an, welde Eei ifrer 2nfunft ans (Englano er= frouftert.

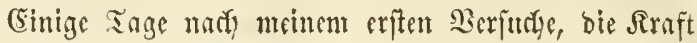
Der Dampfianer bei ben Bferben auzumenten, fam in

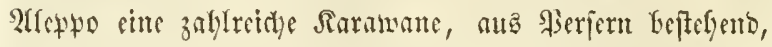

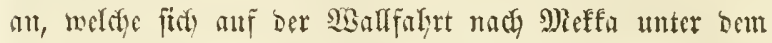

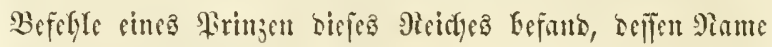

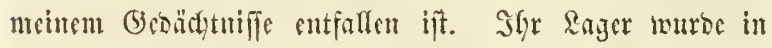

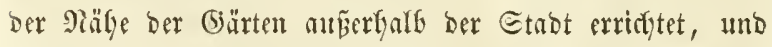
ba fie eine grofe Menge Pferde mit fid füfrten, jo waren mir, ferr won Portes und its, begierig, bieje Thiere zu befurtyen; wir menbeten unê baher an bie an= jelbulidyften Sierionen Der Rnrawane, Die uns mit Der

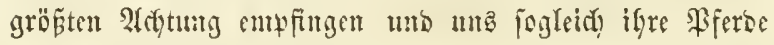
zeigten. Ilnter benjelfen fansen wir uur fefre menige, die einigen Borzug verbienten; die Miefragly mar ftare, wou fdymeren Gliebmapen, Iang von \&cib uno nidft Yeidft; ber Frimg allein Gatte einige von wirflity worzäglidyer

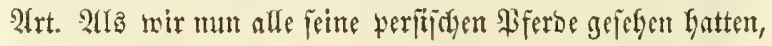

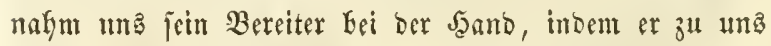
fagte: "Jebzt fommt, um ein arakifaes Fiferb zu jeforn." Dieje mit einer $\mathfrak{2}$ ret Feierlidfeit gefprodyene $\mathfrak{A} \mathfrak{n}=$

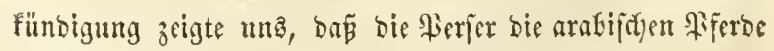
weit mebr adytem, als bie ibrigen, obgleid bie Ietgteren 


\section{3}

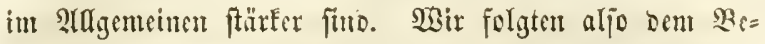

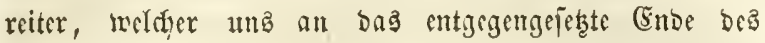
Lagers füfrte, tro er utb eitur Hleinen Braunen yon

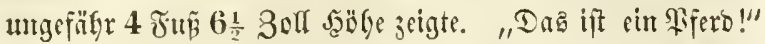
rief ar mit Begeifterung; "reite ç usto Du wirft felyen,

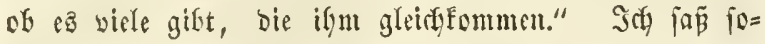

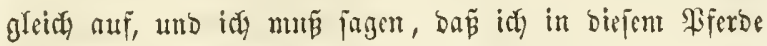
fefgr fäjü Eigenfidaften fanto.

Der Bufalf füfyte an bent mefmulityen Tage cinen

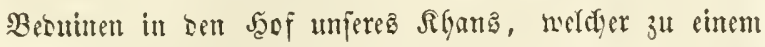

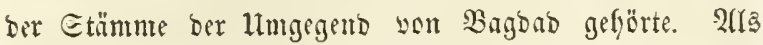

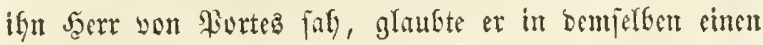

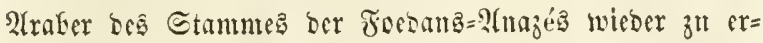
Fennen, ben wir efen verlajien lyatten, und meldyer bie

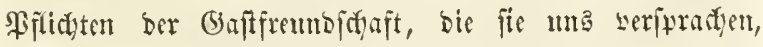

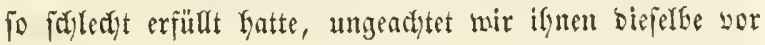
unferer 2 (breife yon 2 (leqpo in Siajtem ober guten

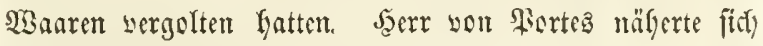
iffm aljo zientlidy zortrig uno warf ifm in jefre lauten

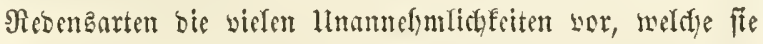

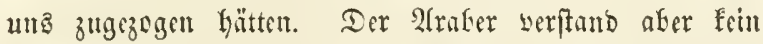
SWort Franzöjitich. Der zornige Ton des Etallmeiffers, feitte beferoen, bic immer rajater unb heftiger murben,

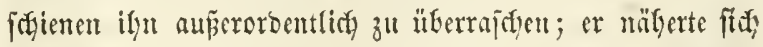

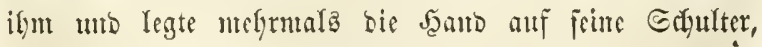

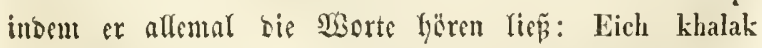

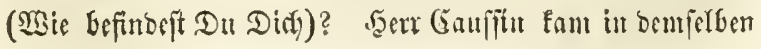


2lugentricte baju, an ben fith ber Etallmeifer jogleidy

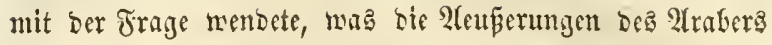
bebeuteten; ricjelfen murben ifm erflärt:

"Sat) glaube, er madyt fid üfer ntid) lujtig," jagte Şerr won Siortes; allein Der Arafer wieberlyolte melyrmals, slone fidf ftören zn Yajpen, jein eich khalak. Sa wein nidyt, พas aแล biefen fonderbaren 2fuftritte gemorben märe, menn nidyt 5̧err Caujïn ben erfitaunten $\mathfrak{A}$ raber ge=

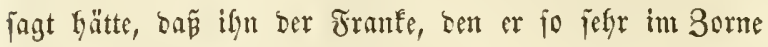

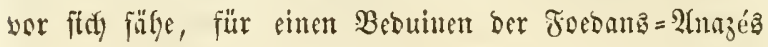
anjehe, in Deren Stamme er einige Beit zugebracht und über ben er fid jefr zu beflagen bätte.

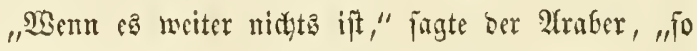
mü|jen wir, biejer Franfe mo ida, bie bepten Freumbe ber SBelt fein, ba die Focoans meine graujanten Fcinbe finb, uno idy fie mefre lafje, als ę irgend je cin (Suropäer im Etanbe iff."

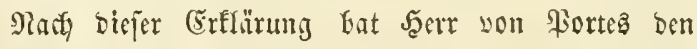
Bebuinen, einige (Erfrifaumgen bci ifm einjunefimen, woranf fict Beibe in Geften Bernefymen tremuten.

Im Raufe biejes Tages waren bie łerjer verbunben, afle Tüdyer, Teppidye und andere Serfaufzartifel, bie fie mit fid gebradgt Gatten, im 3olfgaue niebcraulegen.

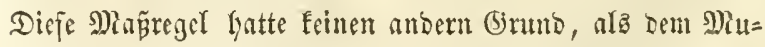

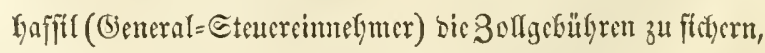
weldye er yon jedem begenftande erfyebt. Sicoer תauf=

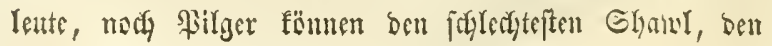


geringiten Tequiff verfaufen, obme fie zuvor im 3olfgate niebergelegt umb bie 2legaten erlegt zu hafen. Dieje Alggate werüntert fid und ber Tare, weldye über bie

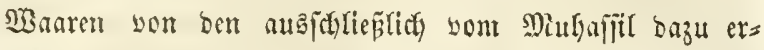

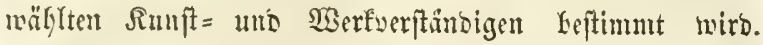

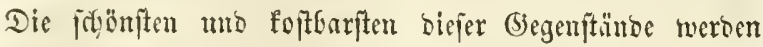

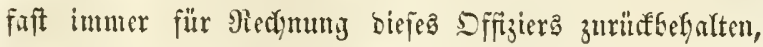
Der, wie man leicht benfen fanu, mur jeflr menig Belb bafür gift.

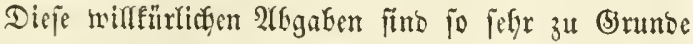
ridfent, Daßs

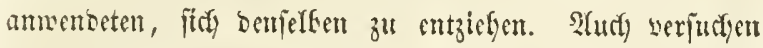

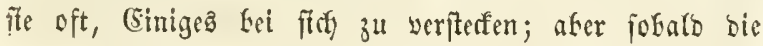

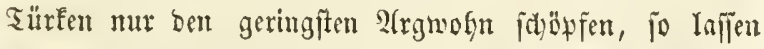
Tie bieferten nauft ausfleiden, unb entoeffen fie ben $\mathfrak{B}_{\text {etrug, }}$

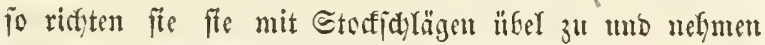

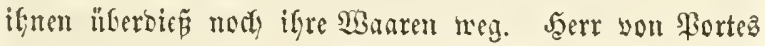
uno id, wir waren mefrmals Zeugen eines 2 uftrittę biefer 2 rrt.

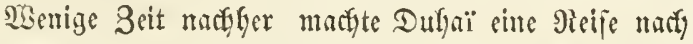
Mlleffo. (Er murbe son Daub begleitet, welder mir ver=

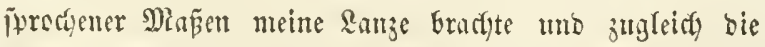

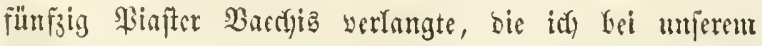

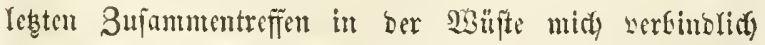

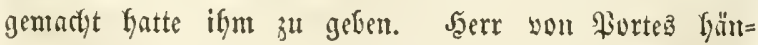
sigte ifm biefelten ein, aber nicyt obne ifym rebhafte

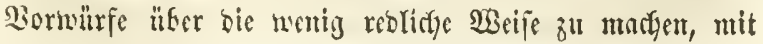




\section{6}

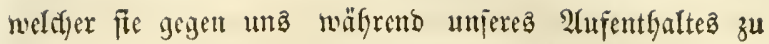
Tel=el=Sultani gefyambelt hatten. Dulyaï fam barauf aud Daju; Der Empfang, ben wir ifym ermiejen, max nidyt viel frembidyaftlidyer; Denu ber Stallueifter jagte igm,

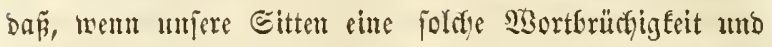
Robgeit geftatteten, wie er fid gegen uns, feime Gäfte uno Freunde, erlanbt bütte, die fith in Folge ber $3 e r=$

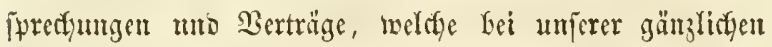
Berlafjenfeit gerabe um jo Geiliger bätten gefyalten werben nüifen, jeiner Madjt übergeben gätten: jo fönnten wix

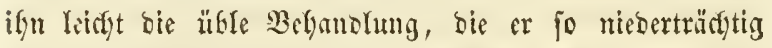

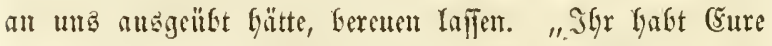

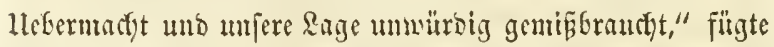

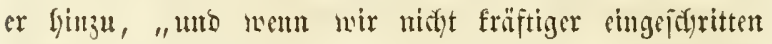

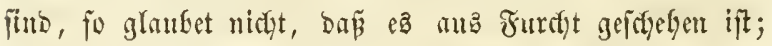

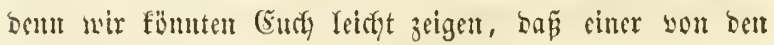
Infrigent nidyt yor zweien ber Curen zittern wirbe. SBenu Du jelbit nori) Geute ben Berjuch madien millf," fagte er, jid perpönlidy zul ben Gateif mentent, "fo

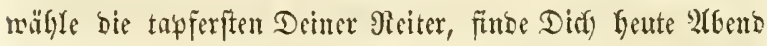
auperfyalb sen Ctabttyoren ein, und id werbe mit eincr

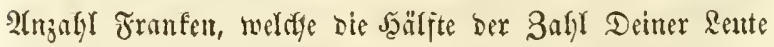

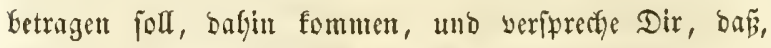
ment ein (Eimgiger you Eud) bavon fonmt, es mux ourda cine Flud geforgen famm, bie jebe andere an Erfurlligfeit übertref̃en müpte, beren (sure \$lündererbanoen bie \$Büfte oft zur Bengin madyen." 


\section{7}

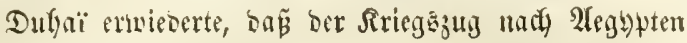
sie frangöfije Tapferfeit feimem Beifte nur ju tief ein=

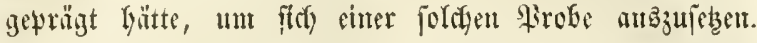
Bon allem unjern $\mathfrak{B a f f e n ~ w a x ~ e z ~ S e r ~ D e g e n , ~ b e n ~ e r ~ a m ~}$

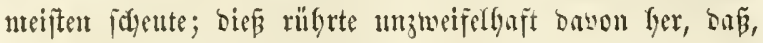

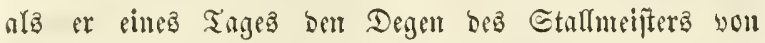

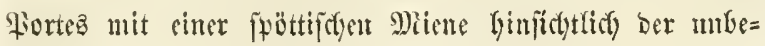
Deutenden Läuge uno ber Reidytigfeit Ser Slinge Getradytete, idy ify gefagt batte, oné, obgleicy bieje SBarje meit für= jer tmo weniger fturf wäre, als feine $2 a n j e$, ing fo $b_{e}=$

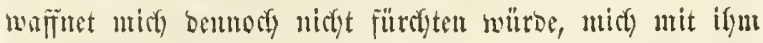

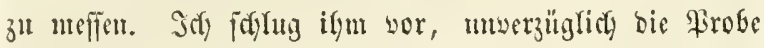
zul madyen, worein er and elmmifligte. Sid ftellte mids aljo wor ifyn biu, unb bei ber erften Bemegnutg, sie er martyte, um midy zu ftopen, war ein leidyter Degentyieb finlänglidy, ifm feime Ramze jeitmärtz abzujd)lagen; idy fprang fogleict) näber mb fetzte ify bie Spibe ber Rlinge

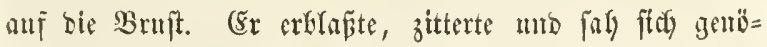

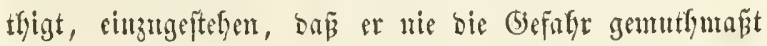

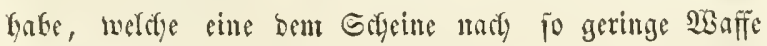
Gieten finne.

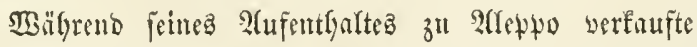

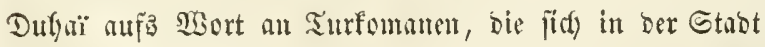
Gejamben, mefre Rameele, weldye er mit zwei Bebuinen

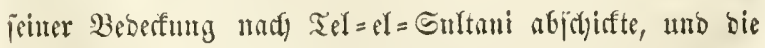
gerabe biejenigen waren, wou benen idt) fo viele Beidfen

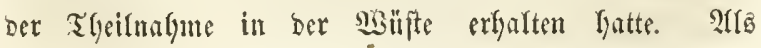




\section{8}

biejelfen auf ifrer Rünfefor aub beur Rager faum bie

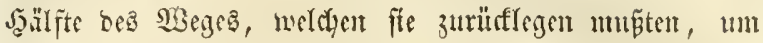

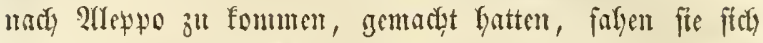
burd) Germmirrembe 2(raber eimes andern Stammes ange= grifien, melde ifuen igre Ramedle und Rlcibungen ab=

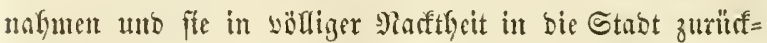

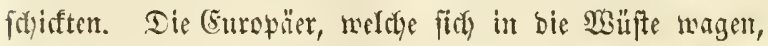
find oft llufïlen biefer 2lrt ausgejegt; benn int 2lugen= blife, wo uran ç ant menigften erwartet, ftïrzen bieje Säubertyorben mit einer folthen Edynelligfeit baber, bap nan faum Seit hat, fich melyrbaft zu marten; eben fo fituell fliefon fie audy. Steflt mun ifyen feimen Wiber= ftrmo elitgegen, fo begnügen fie fich genteiniglish, ben 2 nn= gefaffenen zu befteflen unb narft auşzujichen. Sommt oam ber Bernubte zufïfling eimer andern Tmppe in bat Meg, melde fict) berjelben (Smerbarat mibnet, fo fietet bieje bemjelben (5ajtrembictaft an tmb bebefft ifn mit rincm idjlethten Diantel ober mit Sleibern, meldye won lungeziefer wimmefn. Seben Tag fieft man in ben

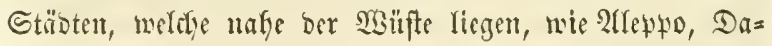
maลี fdaen anfonmen, neldye, Syfer Dergleidyen Begegniffe, in wollfommenfer Sarftycit burdy bie Strafen Iaufent. Man ift aber fo febr baran gemögnt, dap̃ bie Boriber= gefiemben midyt im mimbeften barauf actaten.

Eatyon feit langer Beit ermartete man zu Sllepto

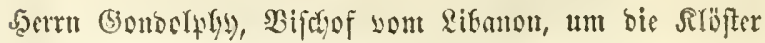


ber Strot jull Gefititigen. Sobald ber Tag feittes (Eimzuges befaunt geworden war, nafment alfe Franten 2lleppo's an ber feierlidyen (Einfyolung befferten Theil.

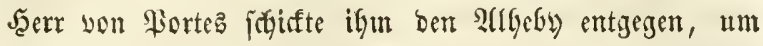
auf bemietben in Die Stabt cinzuziefert. Der $\mathfrak{W}$ Geg, wel= dyen wir burdfritten, war mit Mämern un๖ Frauen yon allen Mieligioneu bebeft, unter benen man eine gra $\tilde{E} e$

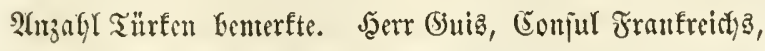
ritt an ber Spitse ber (Europäer und ber Baratairea ")

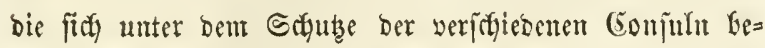
fanden. Der Stallneeifter won Fortes folgte auy bem

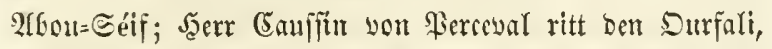

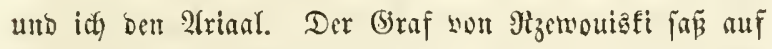

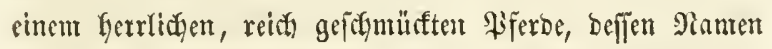
idi midy nidyt mefre entfinne.

Ş3ir trafen ben Bijtgof in ber grofent Rarawanen= Gerberge zи 2(njarz)e, eimem fleinen Dorfe mrgejüfyr zmei

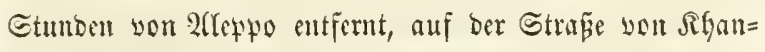

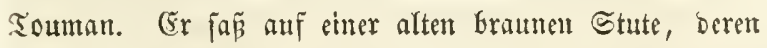

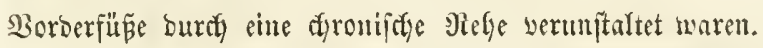

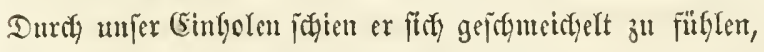
nafju afer mur auf vieles inftündiges Bitten bas Fifero au,

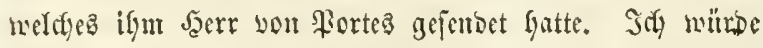

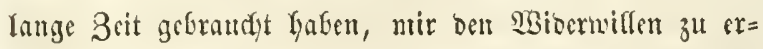

-) (Singeimifisfe, meiffentfeitz (GGriften ober Jubcn, weldace

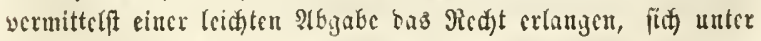

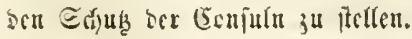




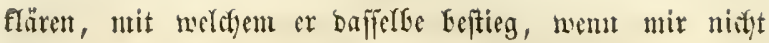
nachfer CGriften be

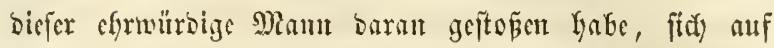
cinem fiferbe mit etwa furjem Exymeife zu zeigen, da rie Ränge ber Şare in bicjemt Rantoe cin mezertrennlidjes

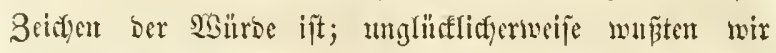
afer bavou nictot?.

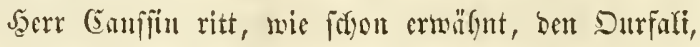
welcher fo foffr aufjeregt war, ba

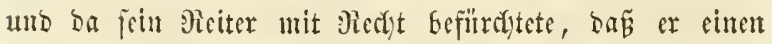

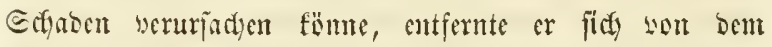

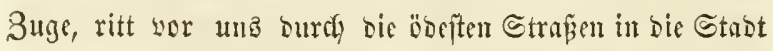

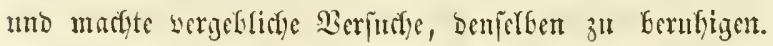

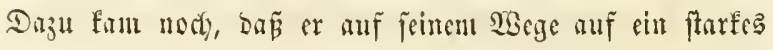

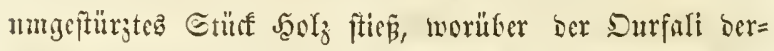

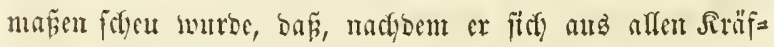
ten gewefhrt Gatte unt mefre Male gefitiegen war, er fich

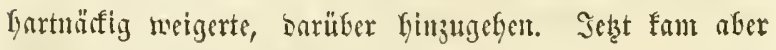
cint alter Edyerif (2lefömmling won Matgomeb), weldyen ber 3nfall hicrler füfrte; er gab Şern Cauffin cin

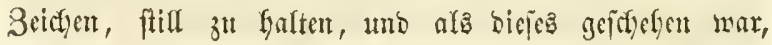

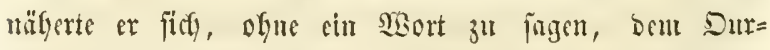

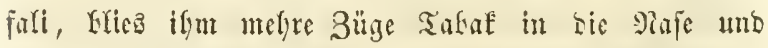

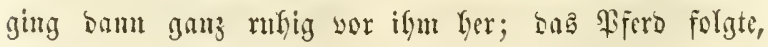
ging Eei beul begenftanbe, ber ifgm cinten fo grofen

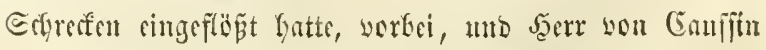

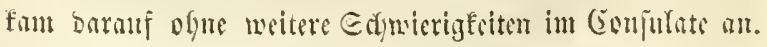




\section{1}

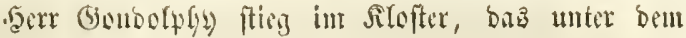
Paunen, "Des Geiligen Rambes" Gefount ijt, ab unb blieb einige

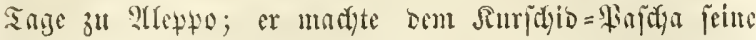

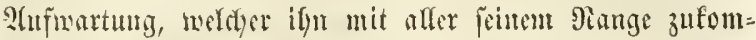

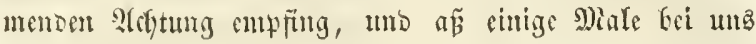

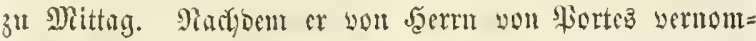

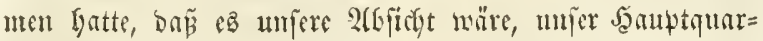
tier mad) Geiba zn verlegen, um bie llmgegentoen won Damaвfuz umb Den Ribamon bejuchen zu fümen, fot er

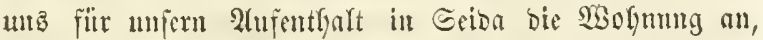

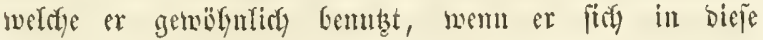

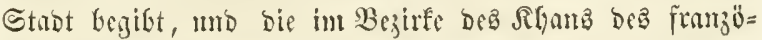

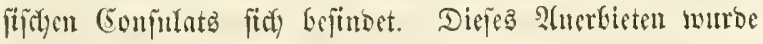

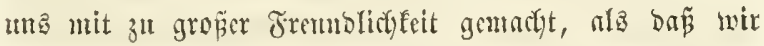

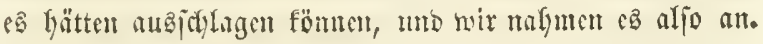

Misir fanften nody sintige Prerbe, obgleidy bie (berfte

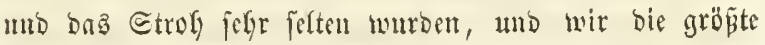

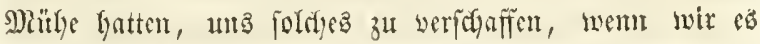

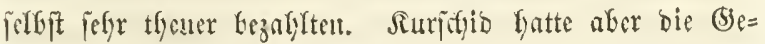
fälligfeit, uns einen geniffen Theil geben zu lafien, wel= dyen er you ben Naturallieferungen, bie er bautale auf alle Dörifer ber Unigegend regte, entunfum.

Seer you Portez badyte num ermftlich baran, maty Saiba

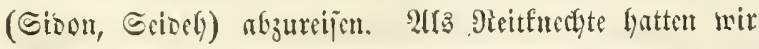

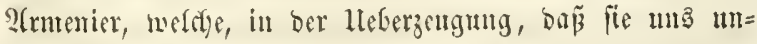
entbefrlicf) wären, glaubten, ummäpigen Gold fordern zu

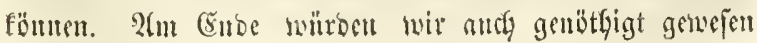




\section{2}

feir, in ifye forberutrgen zu wifliges, wern unz nidat Surichib, bem man unjere Serfegenfeit Ginterfaratite, vor=

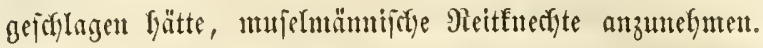
Der Etallmeifter bütete fict mohl, bas Manerbicten auBzlt=

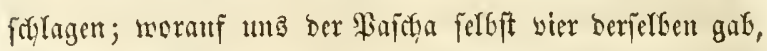

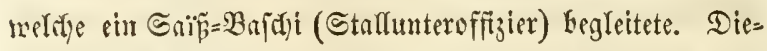

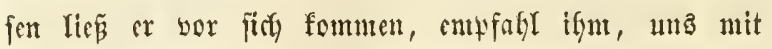
Treute zu bienen unto Gorge z"l tragen, ba ofue lunjall für umb volfenbet müros. "Dut ferffit mit Deinem תoffe für bieje Franfen," fagte er ifgm; "bafer

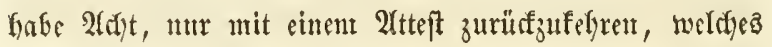
Deine guten Dienffe bezcugt."

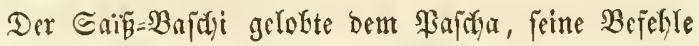

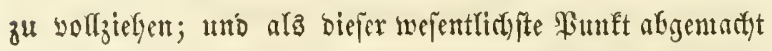

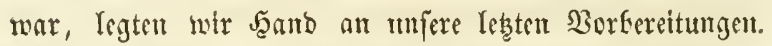

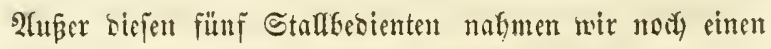
armeniçyen Diener, Namens Sfutonio, nit uns,

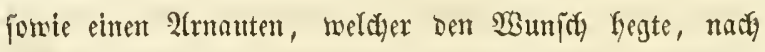

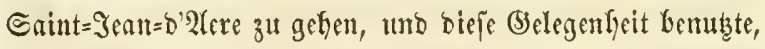
um mit mefyr Sictjerbeit zu reifen. Diejer Mamr, ber

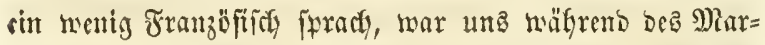

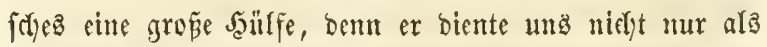
Drogmann, jonbern fitfrte autd eins umperer Prerbe.

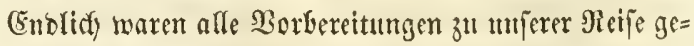

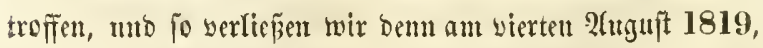

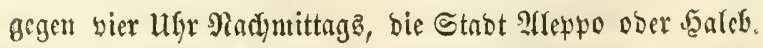




\section{$\mathfrak{A d}$ tes $\mathfrak{R} a \mathfrak{i t e l}$.}

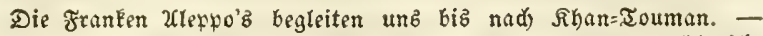

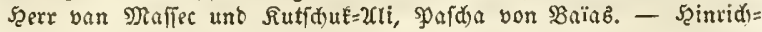

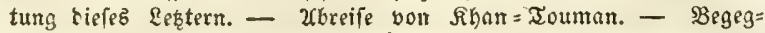
nungen unt grobe Şik̨e in ter 23 iffte. - Unkunft zu Sermein. -

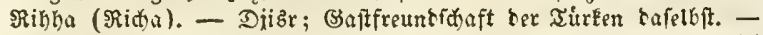

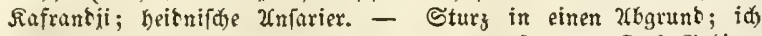
brode รas Bruftbein. - 3olhhau von (5hafar. - Daz Bebirge Setłub. - SBehloutie. - 2rnkunft zu Eatakia; 2ufenthalt Dafelbft. - Mteudelmorb eines jungen Ehriften; fein Tob u. i. w.

Indent unjere Raramane bie TGore Aleppo's ver= lief, beftanto fie ans einem Monfer, welcher vier mit un= forem Sewär belaftete Manltyiere fübrte, tnd aus jiefen= zefhn Mlännern, beren jeber ein Fifero an ber Sant hatte.

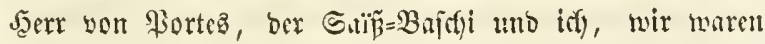
bie einzigen Berittenen, unb won bent ganjen l̈brigen

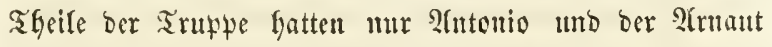

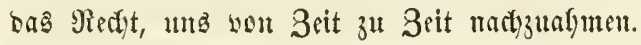

Ұrfe Confuln und Franfen Der Stnot Gegleiteten uns

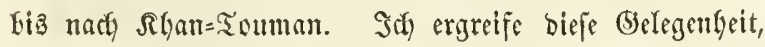

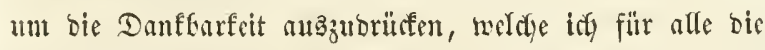

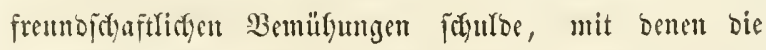
Melirzabl .Serrm v. Portes unb mir entgegenfauten. Sn meiner Berlegenfeit, um allen zum framzijificten (Eonjulat

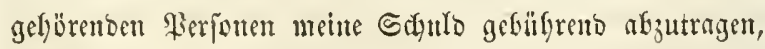
ocren Namen fifjon mefr alz ein Mal won nteiner Feber genannt worben ftutb, werbe ict) mict) Gier begnïgen, bie

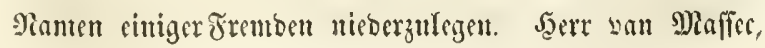




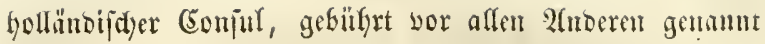
z" weroen, necleyen feine Serbältuifife zu ben meiften tur=

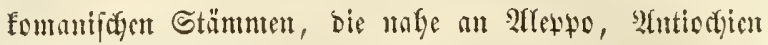
unb bie sorjinglidyften Rilftentiäbte gremzen, in ben Stand

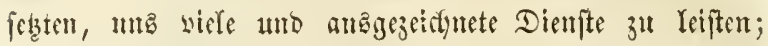

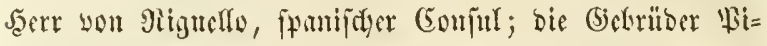
thioto, alfe vier ber jübijden Piefigion angerlyörig und

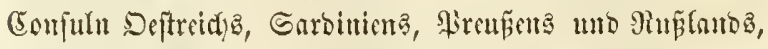

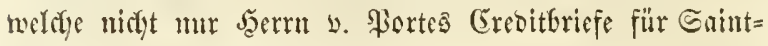

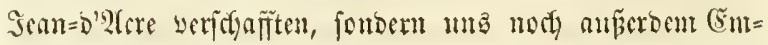

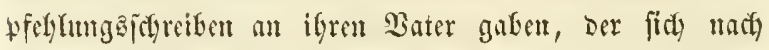
Saphad jutrürfgezogen hatte; Tlaron, ebornalla Siraclit,

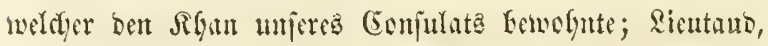

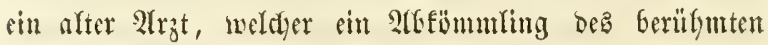

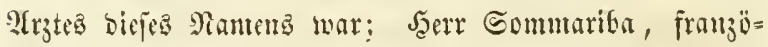

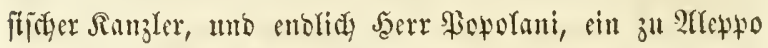
geforener $2 \mathfrak{r}$; auf meine DanfGarfeit; weder bic Beit, noch bie Entfer=

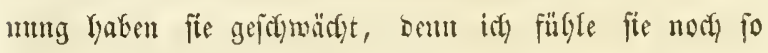
lebhaft in mir, als am eriten Tage.

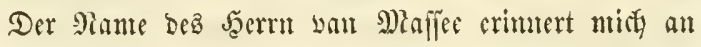
eine Begebenbeit, meldye für bie Sitten Des Drienta zu Eezeifynento ift, als ba

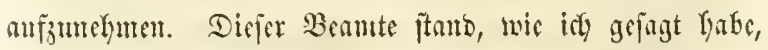

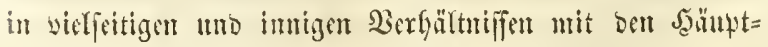
lingen Der Städe uno ber Stämme, bie in ber gam jen

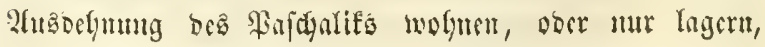


und welde er of̣t bejucte. So fïfrte iqu audy cine

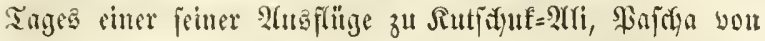
Boins, eimem feiner beften Freumbe. Diejer empfing ifu

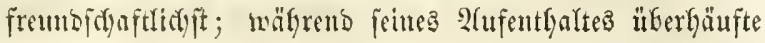
or ifn mit Stworfommentyeiten aller 2 irt unb ben $\mathfrak{B}_{e}=$ weifen ber anfridytigften Bumeigumg; als er aber abreipen

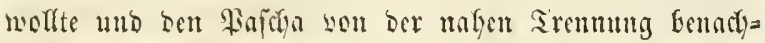
ridftigte, antwortete ifgm biejer, ban er nidyt baran benfen folte, jo jounefl won ism gebon zu fömen, inden er fein (5)cfungener wäre, uno er niçt eber afreifen fömnte, alb bis or ifn cine bebentende Gumme beloes eingebändigt

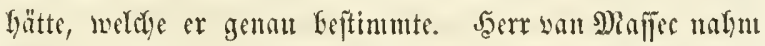

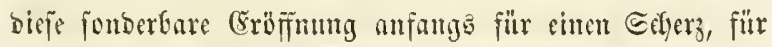

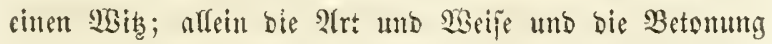

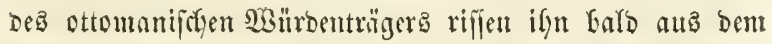
Srrtgum; baber mollte er ficty mun befragen.

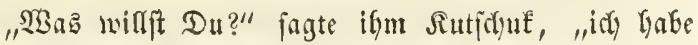
(Geld nötyig, uto wen foll idy Darmm anprecten, als meine Freunde?"

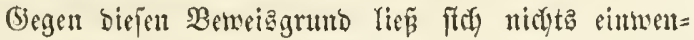

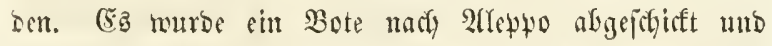

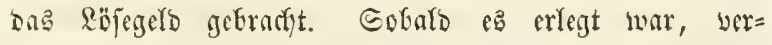

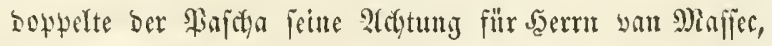

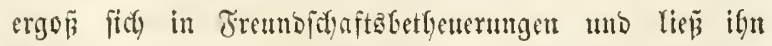
cholidy fret aut feinem Salfajte geben; Secr wan Mlaffec judte ficts ciner fo foffipieligen Frembjofaft zu entziefen

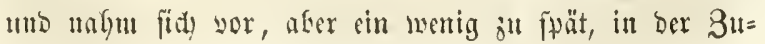




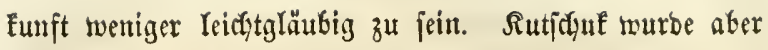
bald felbit cin Dpfer ber türfiffyen Freundichaft. Das

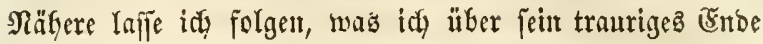
gejammelt Gabe: Melfre Male Gatte ifin ber Sultan afs= gefeşt unto jebes ltrtheil mar von bem Frirntan bez Todes begleitet geirefen, welderer in ber Türtei bem Gdyuldigen

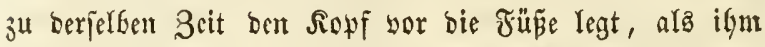

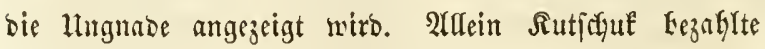
cintige Solticber bez Divanz reidflid, mogegen bieje ifgn

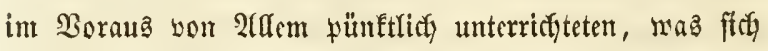
gegen feinte $\mathfrak{R a d j t}$ ober feitr Reben ampintuen fönnte; umb menn fich nun ber Tobeåbote zeigte, überlyüufte or ifgn

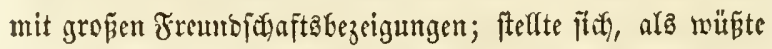

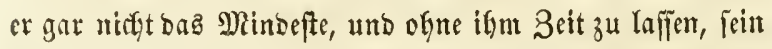

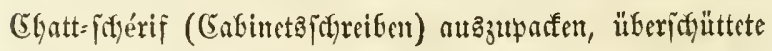

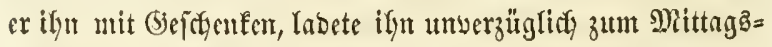

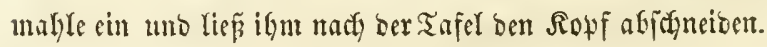
Diejes Siegeszzeichen wurbe foglridy ber Pforte gefendet, meldye, beffere Gelegenfyeit abwartent, nie unterlié, ben wer=

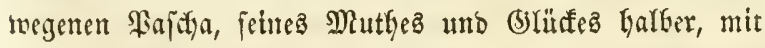

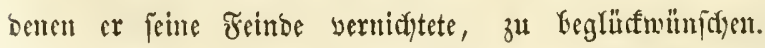
Mielyre (5apisj= Söpfe maren fdyon im Serail angefomuten,

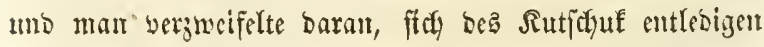

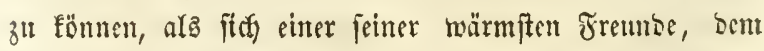

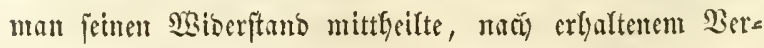
iprectyen einter grö́n Belohnumg, erbot, ben Gultan you

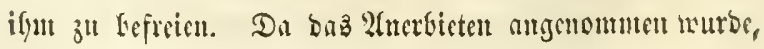




\section{7}

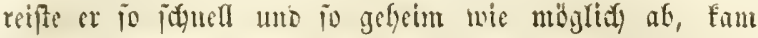

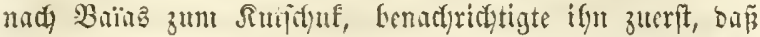
cr mach Beirut getgen wolite, und theilte ifgm bann, iffn

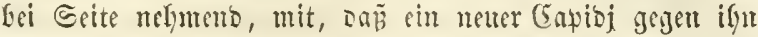

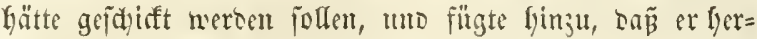
Geigeeilt wäre, ifn bayon zu mterricften, forve ifn zu Eitten, ja auf jeiner Sat zu jein, uno baf in ben Falle, mem ber Bote zur Brit jeiner अïuffefr nody nidjt ange= fommen märe, or ifm bann auf jeinem Gdifĩe eine $3 \mathrm{Wu}$ fludt anfiete, weldye ifn und jeine ganzen Reidytyümer

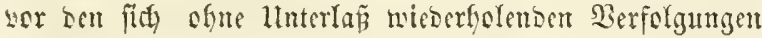
ber Sĩorte in Eidyerfeit fringen mürde. Der leidytglüt= Gige Sanja crgof fich in Dankiagungen umb betfouterte, an jeben Gejamoten bes Eultans, Capisj ober niclit, gute (5eredtigfeit zu itwen. Rach Serlanf son einigen Tagen fau fein guter Freuno wou feiner vorgegebenen Эieije ju= rïf; allein nod! Miemant fatte fitty gezeigt.

"Tiefer Eapibj wirb shute 3meifel cimige Serjö= gerung auf jeiner Mecije erlitten baben," jagte ber Rebtere zit Rutichuf, "aber or mun balo Gier jein; bie fllugfeit getietet Dir bafyer, an meinem Barb zu jalajen."

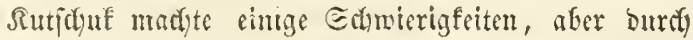
bas Buresen bes Ginterlifitigen Türfen beftegt, bradyte or elle feime Jieidythimer in Dromung unb fam cnofich an

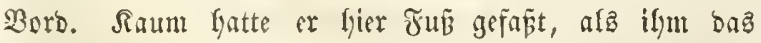

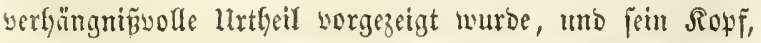
ehe ex baran benfen fonnte, fid zul vertheibigen, auf bas 


\section{8}

Berbeaf nicberfiel. Sein Sïrper war zu Baylam, gerroc cinige Tage wor umperer Lanbung zu 2Aferanbrette, beerbigt morbent.

Das Pferb, weldyes ber Stallmeifter yon Portea ben Minteferth won Rilliz afgeffuift hatte, war you

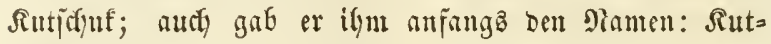

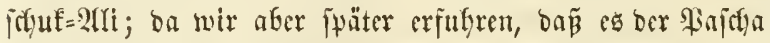
aus Meffa gefradyt hatte, gab ifm ber Etallmeifter, wie idy) bas idgon früfer gejagt zu haben glanbe, ben Miamen Miffermi.

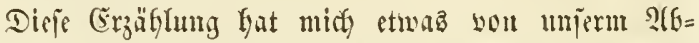

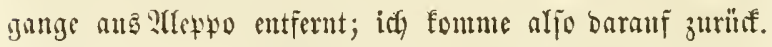

lluter ben (Ěmropäern, bie uns bis nad) $\mathfrak{K}$ gan=:Tou= man Vegleiteten, Gefando fith Ler (5raf $\Re_{3}$ emouiafi, weldyer

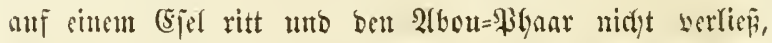

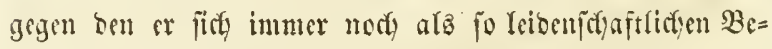

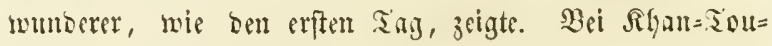
man angefommen, bieften alle bieje Șerren an, malymen

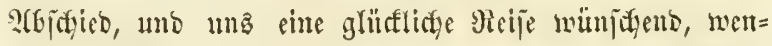

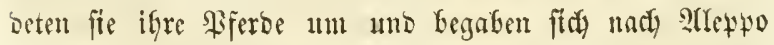

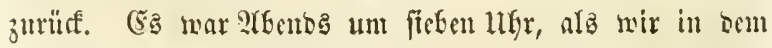
Dorfe anfamen. Sisir ließ̃en unfere Belte bei ciner ziemt=

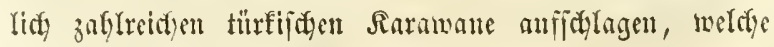
bier gleidyfalls bie Siadyt zufringen muṕte.

Den fommenben Tag begaben mir uns beim 2hif= gang ber Eonme auf ben $\mathfrak{W}$ eg nady Germein, finem

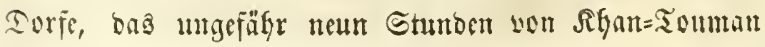




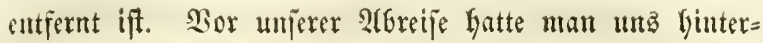

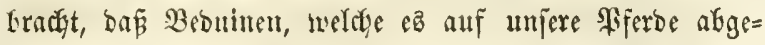

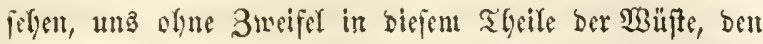

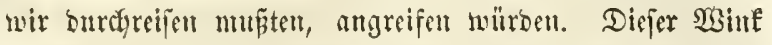

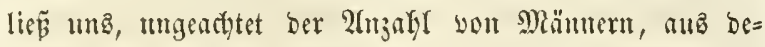
nen unjere Raramame beftanto, auj umjerer Şut fein; bent obgleict mir für unjere ganze Trupte vierzefn Jlinten,

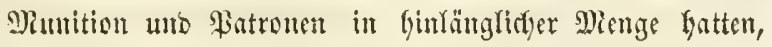
um ein iffarfez fouer $z^{u}$ unterfyalten, fo maren wir ben= nod), menn idy as geftefjen foll, nidyt ofyne lebgafte $\mathfrak{l n}=$ rutye. S(utr) Gatte idy, wem fith ber fleinfte begenjtanto num Serizonte zeigte, nicht Dringenderes

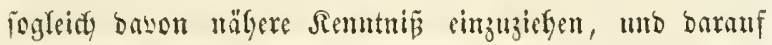

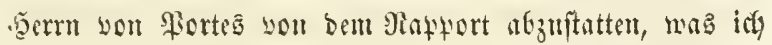
gefeffen hatte. (Sich ritt an biefem Tage ben 2(rianl).

(Eime geraume Beit war werflofifon, wälyetto weldyer wir ofue unverwutheten Rärm maridjitt waten, ars idf wlöbzlidy ben Meufer umb bie Füfyer unjerer \$ierbe, alfe auf cimmar, rufen fyörte: Ada el Bedawis (ba fitno bie

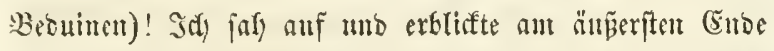

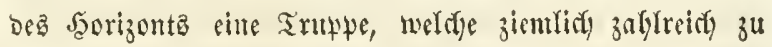

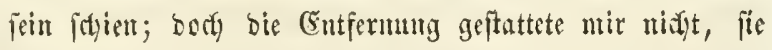
3th erfentert. Der Etallmeiffer befond firfy serabe weit hinter ber Saramane mit bem 2(Gou=Ffyar, meldyer ben

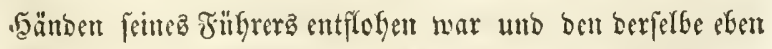

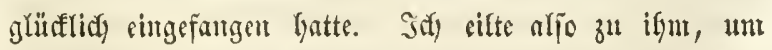

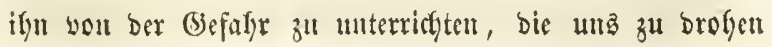




\section{0}

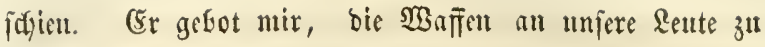

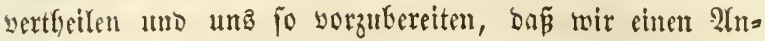

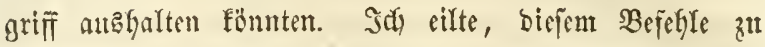
genügen, alleiu, als idf mit ser Bertlyeilung ber Flinter cinen Anfang martete, zeigte ficts bei ber ganzen Truppe cin Wiocrmille, fid zu betwaf̃un, aus weldyem zuleb̧t cine förmlitye QBeigerung wurbe. Reimer biejer Rerle hatte (Stmas zu verlieren; baber ichte id soraus, bafip fite Den 3orm und bie Siadje ber Bebumen nidyt buref eitten Natoerftano reizen mollen, sen fie für sergeblitit Gielten;

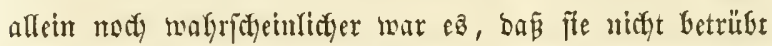
gewejen fein whiroen, bie Saramane zeritreuen zu fefsen, unzweifelfaft hof̃end, fid étuiger ber TGiere bemädutigen 3u fönuen, die ifnen ansertraut waren. Diejer veroricä=

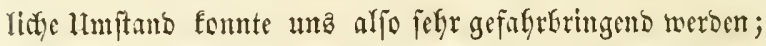

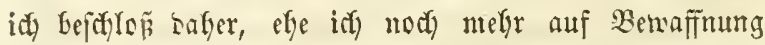
oranty, mid feldit z"ll

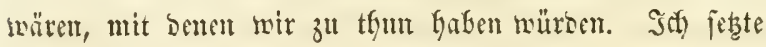
beide હporen ein und erfannte Gars in ben vermeintlitfen

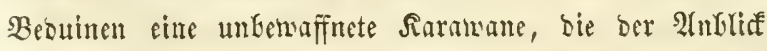
mijerer Siferse mit હdurecten erfüllt Gate. Innergüglict) bejdileunigten wir num unjere Ectyritte uns erreidgten fie Gald. Dieję Sujammentref̃en war für uns ein fefor glübfidyer Itmptand, benn menige Augenblicfe barauf zeigte

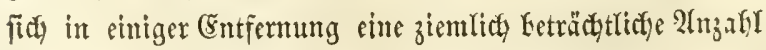
Sraker; allein burdy unjere Menge erjedredt, magten fie nidut, unz lange Seit zat folgen, und entfernten fidy barauf. 


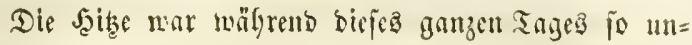

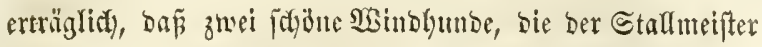
won Forteß mit fid) füfrte, vor Erichoptrutg unb Mangel an Staffrung untanten. Dor Cinguèe, jener Şünbin, welde nir Colafiz gegeten hatte, war ber Siüfen wou ber Somne gänzlich verbramnt; fie fängte bamala zmei Sunge, Daud und Dafer: Gelise mürben unfeflyar baben

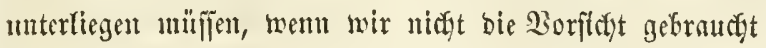

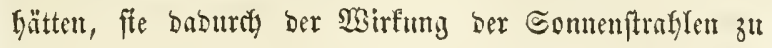

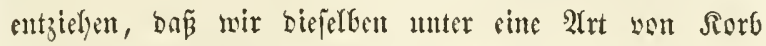

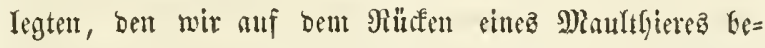
feftigt ratten. Die Sefftigfeit ber Saibe war aut biefent

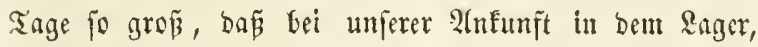
wo wir üferuadjens nollten, afle umpere Fferbe, bie=

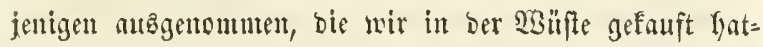

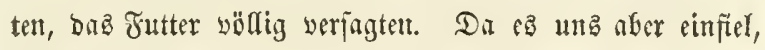

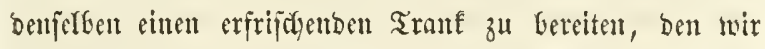
erffieften, ittom mir Şaffer mit ciner gewiffen Menge fautrer Mild verntichtem, bie wir ums in Dem Dorfe zu

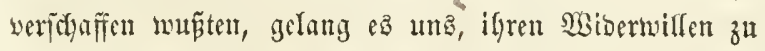
überminden, denu fite fofifen biefes Bjetränf mit vieler \&uft; welcher (Erfolg ums ermutfjigte. Das ando erzentgt

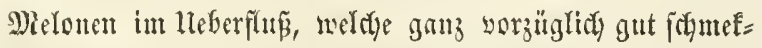

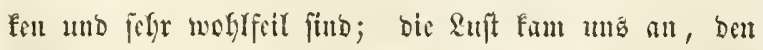
Bjerben Davon anjubieten. Diejer newe Berjunt) glinfte

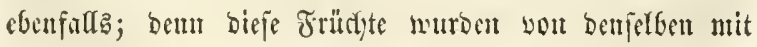

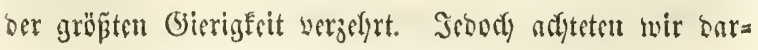




\section{2}

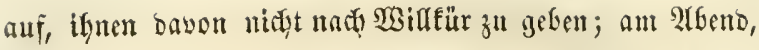
Geim Luntergang ber Soume, reichte man ifmen Gerfite, unb ben Mlorgen barauf befanden fid alle im Stande,

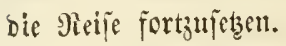

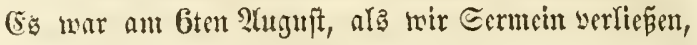
Im nach) Jiblya, ciner fleimen Ctadt, vier Stunden $\mathfrak{W} e=$ ges von Eermen entfernt, zu geflent. Sie liegt füblicid won cinem fleinen Berge; im פoroen beffreltern und zwar in ciner Cutpernumg von etma jmei Etumber liegt Joleb, sine Etabt, bie nidgt ofyne bebententoen Samber ift. llm

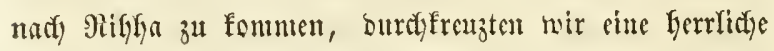

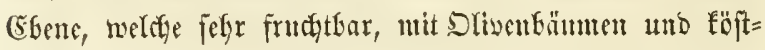

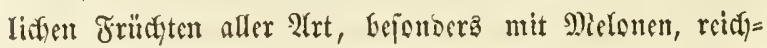
lich Geoefft mar. Diejer Tag verging ohne mittlyeilumgs:=

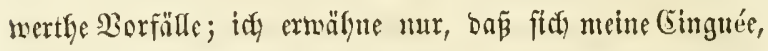
weldye gef́tern einem fafít genififen Tobe entgangen $\mathbf{m a r}$, biejen Tag nodj fo ermattet fïflte, dá⿱㇒㠯 lids twurbe, ber Raramane zla folgen. Sod war bafer gentötyigt, fie auf cin Maulthier zu jeţent. Die Șaut,

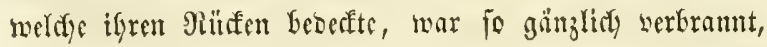
bar fie wentige Tage baranf in Gdyorfen abfiel umb ifyr

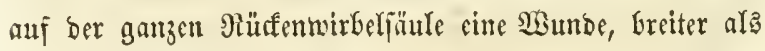
cine Santo, Elieb.

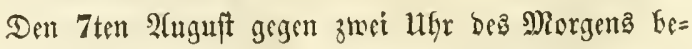

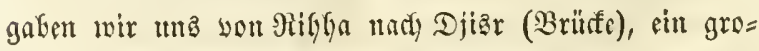

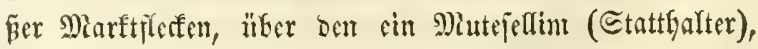

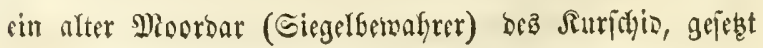




\section{3}

mar, weldyen wir nelyrmalz iu Sallajte bes \$ajdya ge= trofien hatten, und ber แmb alles bas ju verichaffen be= milgt war, mas wir, nidgt allein für un jelbft, fonbern fogar für unjere sente und Siferbe nötyig batten. Djišr liegt auf bent linfen 11 fer bes Srontes; Hacibem wir über bie Brüfe gegangen waren, weldye über biejen

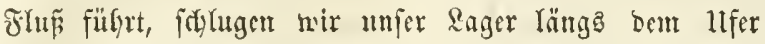

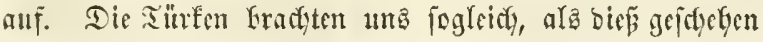

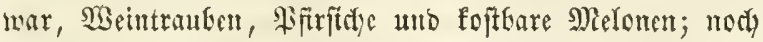
fügten jie diejen eriquifenden Epeifen (5is bei, bas fie aแร Den Genadybarten Bergen begielyen. 2ale bieje গiafy= rungsిmittel maren gemí̄ genügent allein bie gefällige Gaffreundidyaft unjeres SBirtbes Glieb babei nidyt, fondern bereitete una cine no(f) angenefymere Uebernajumg. Der Droutes ift melymlidy jefre fifdreidy: umpere Türen becilten fies, bie- Netze hinein zu werfen, unb zogen im Berlanf einiger Afugenblife 2lale uno jegr föbune Erbleifen in Yyinlänglicter Mlenge Geraus, un bie ganze Raramane zu fättigen. 2(m 26Geno, Geim Ilntergang ber Some, Gatten

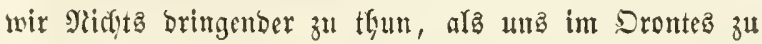
baben und bann alle unjere Pferde binein zu fübren.

Den folgenden Tag, um vier llyr, begaben wir uns

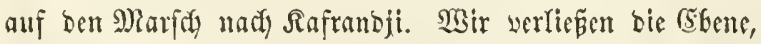
um in eine jogmierige unb bergige Begend cinzugefon.

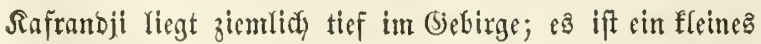
Dorf, bas wou heibnijofen Anjariern bewolynt miro, beren

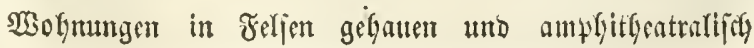




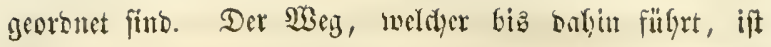
antereroroentlich felfity. Nadbocm nan fogon lange geffiegen ift, fonmt man an chus Flab, ber mit eiuen grïnen

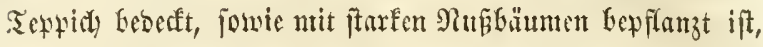
und un bett wan cine ziemlidy betrïdtlidje Babl Suellen

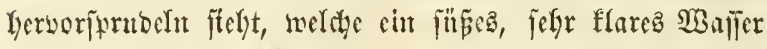

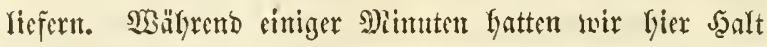

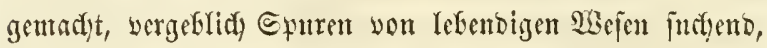
ohne ctwas 2nocres cripägen zu fönnen, als narfte Fer= fen, weldye eften fo viele unzugänglidye Manern zu fein

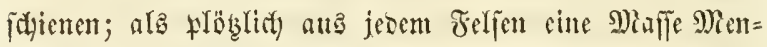
ficyen fanten, weldie unjer sager umringtent und fid beciferten, uns alle Dinge uno Lebensamittel zu bringen, bie wir unt noblgig Gatten. Diejer obgleid bcionijage

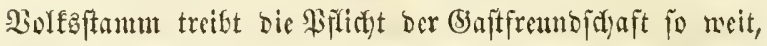

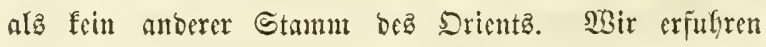
Dawon eitten felyr ftarten Berweis in Dem Betragen feines

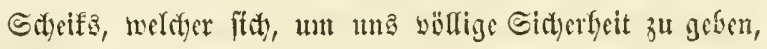
verbinolidy matbte, bie ganze Nacht in mijerer Mitte zu= zubringen.

Hujere nädypte Tagercife brofte felyr lang zu merten,

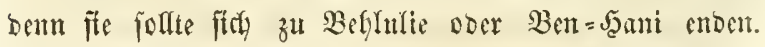

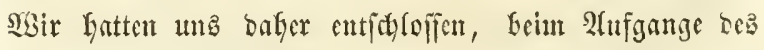

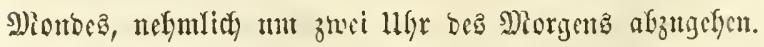
Эd) wefte Galb zwei llgr alle unjere Seute, weldye bie y ferbehananen Beipfërte, die un den Röpfen ifrer Biferbe befeftigt waren, abunfmen แmb bas Rnger nnwer 


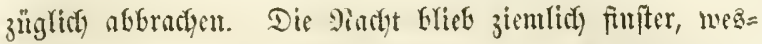
balb fird Der Gtallmtifter an bie Gribe ber Raramane

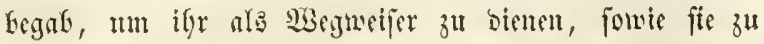

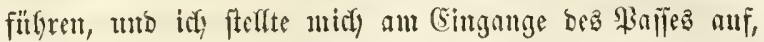

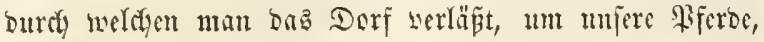
forvie fï bei mir worke faumen, ju zäflen. Daß̉ leţte Sferd Der Trupte war verüber, und idy fand cins zu menig; idi glaubte aljo, mich geirrt zut baben, und becilte mich, wieber an bie Spike bes 3ugez zu fommen, um bas Büflen von neuem anzurangen; alfein eह zeigte fidd

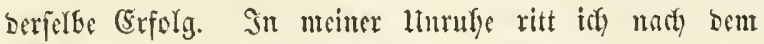
Flabe, mo mir gelagert batten, zurürf unb Gatte Vier bie

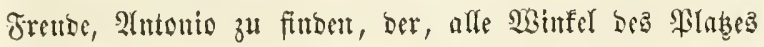

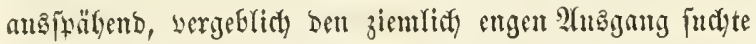
ourdf weldyen wir uts entfernt battert. S(t) zeigte ifut fogleidy ben Sibeg unb beeilte midy, ifm zutorgufommen, um bie Saramane cinzulyolen und bieje bis zu feimer $\mathfrak{P}(n=$ funft marten zu Inffer. Die Nadyt mar, wie idy fagte, ziemlich finfter; idf batte einen fontellen Ranf angensm= men, und indem idy an ber Geite des Tuffteigez vorbei

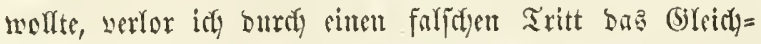
getridyt; idf fiel unb rollte in einen 2logrumb hinab, bef=

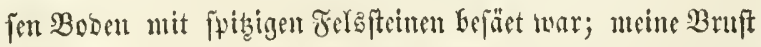
fam gerabe auf cineu berjelben, wobei ber Stop fo beftig war, baß or mein Bruftbein gebrodyen Latte und baz

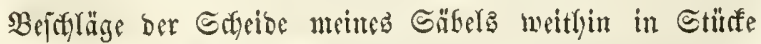

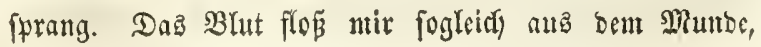


unb id̆ lag einige Seftmben, ohye bie Marjt zu Gaben,

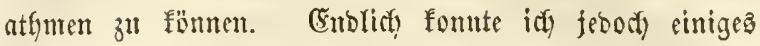

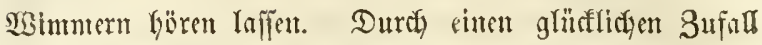

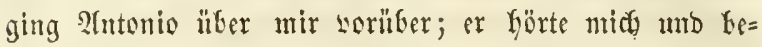
eilte fitid fogleid), io gut er fonnte, zu mir heralbzufteigen

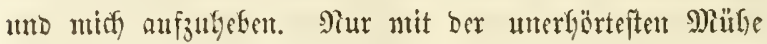

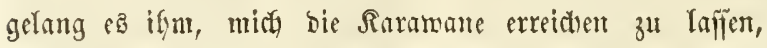

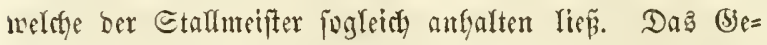

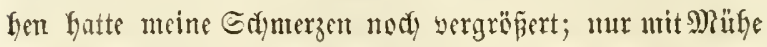
founte id atfymen uno mußste unaufgörlidy Blut fpufen.

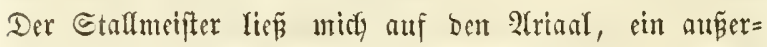

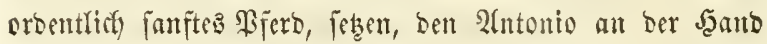
füfrte, weld)er bafür forgte, ifgn nur langjam voridgreiten zu

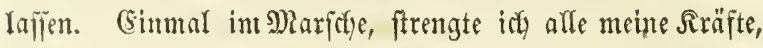

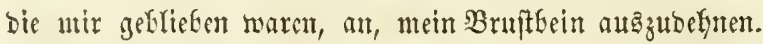
Mieine 2(nffrengungen blieben lange Beit vergeflied; ith

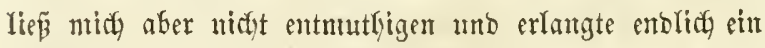

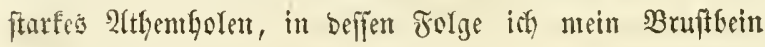

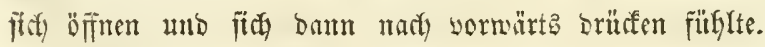
$20 n$ biefem 2 tugenflife an murbe mein 2(thynen freier

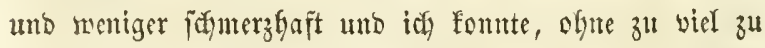
Yeiben, ben Giang neines Bérbez ertragen.

Siath Sorlauf einiger Stunben fam bie fiaramane

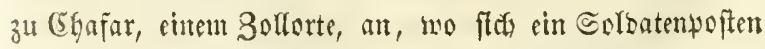

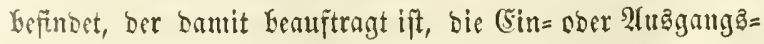

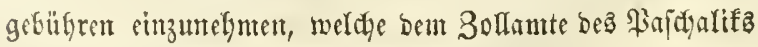
2llepto zufonmen. Sinige unjerer \$iferbe maren bem 
Meple ber Trupte in Geftalt eince Sortrabez vorangegan=

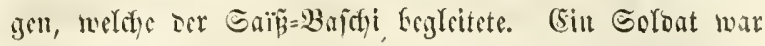
worgetreten, แm ign anzulyalten; ir fagte ifun aber, oañ fith bie Serren ber Saramane weiter Ginten befämben, und

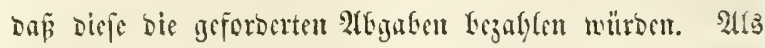

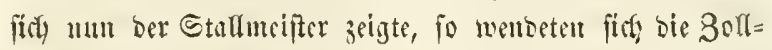
beamten at ifn; aber ber unveridünte son, mit meldyen

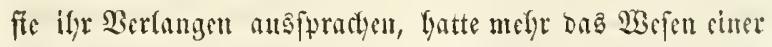
Drolyung, als ciner Forberung. Seer sou Portes, nicht miffero, was fie wollten, entpp̈ng fie fefre itbel; ocr Strcit

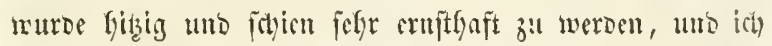

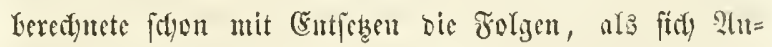
tonio bemübte, 2afcs zu verbeutlichen. Die stggabe murde gezablt, und wir fetzten unjern 2 Seng weiter fort.

Diejer Bwifhentworfall gatte midy fich angegrifin;

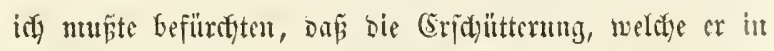
ntir berworbradyte, itble Folgen Gaben mödyte; alleiu fie werminderte gejen ntein (Erwarten ben Gramerz, weldyen idy biager gefüfylt latte, uno shue fefre zu reiden, über=

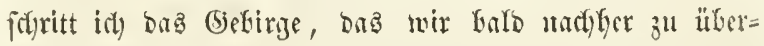
fteigen batterr. Diefes Gebirge twar jeborty feft boch, und

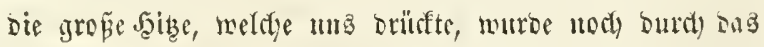
3urücfprallen ber Sommenftralylen von dent weig̈lidy freioe= artigen Boben, Der bie bödjten Sduichten bilocte, wermelyrt.

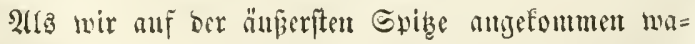
ren, wurde cines ninjerer \$fferbe, mit Sauten (5azul, bas orifiagrig mar, won cinent Fliegenfomarm fo refogit 


\section{8}

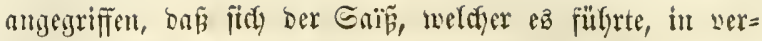

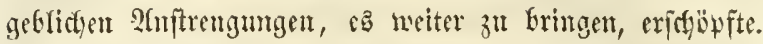
Der Etallmeifter ergriff barmif bicjez Thjer uno mollte ca juingen. Der bazal batte aber einen eigenfinnigen

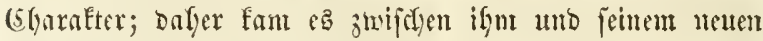

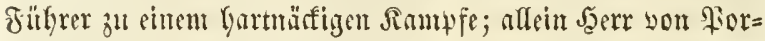
tez Glieb Sieger. Diefe 2 rat won Sinntpf fiel nuf einem

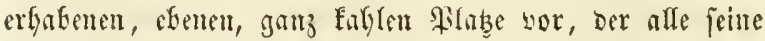
llmgefumgen überragte. Der Drt bieję Borgangea, bie

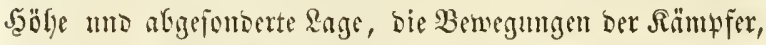

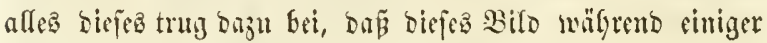
2lugenblitfe bie lebhafteite Theilnafme erregte.

Bom Gifirge Gerafjeigend, begegmetem wir wier Jrauten, weldye, türfijach gefleidet, mad) altewyo gefyen wolfen, un bort Biferde zu faufen. (ss mar ber Mitter

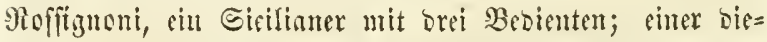

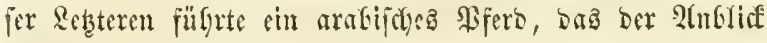
unjerer Saramane fo fegr aufregte, dá̈ mar mit unetblidfer Dï̈fye im Gefjorfan erfalten fomte.

Daz Gefirge, weldfes wir efen üferifgrittent hatten,

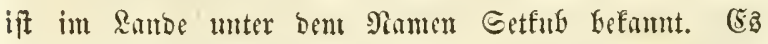

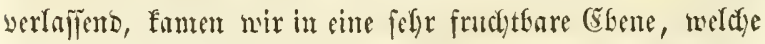
verffyiesone Etämme ber Turfomanen Gemolynen, umb in

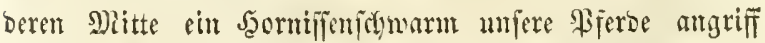

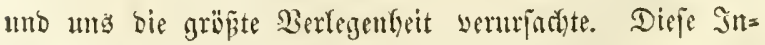

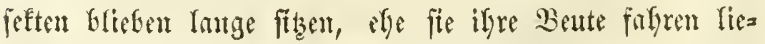
Ẽen, utto erft lautge Beit Daranf, madjbem wir es bafin 
gebract batten, fie zu bertreiben, berubigten fid entidi

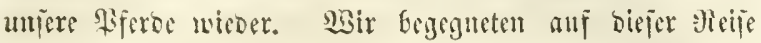

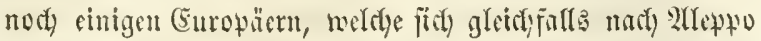

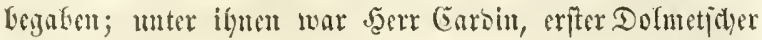

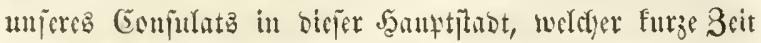
worber bie silijte Franfreidja verlajfen batte.

Nad)bem wir nun nody cinige Etumben geritten wa= ren, langten wir enolidy an Biele bicfez langen 936 ege an, nämlich zแ Bebloulie ober Ben=Sani. Dieję fleine

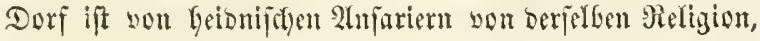
als bie, bei benen wir bie Sacyt zuyor ïbernachtet hat=

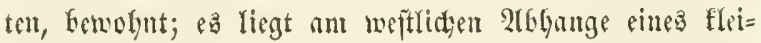

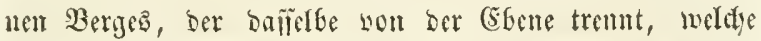
wir burdyreift batten. SBir erbielten won feinen 9 Bemoh=

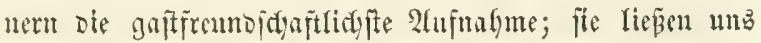
vor bem Dorfe auf Dom Sege maty Rabafia Das Rager

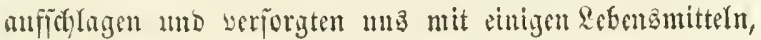
unter benen củt benly war, Das

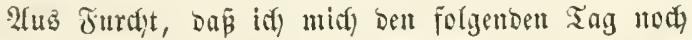
lcisender befinden mörte und Dann bis Aelipe fortzu= fetsen befundert fein würbc, rieth nir ber Etalfmeifter,

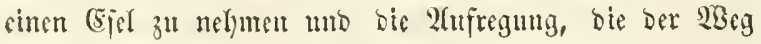
in meinen ganjen Siejen Gervorgebradyt Gatte, Genußeno, benjelfon olne \&ufenthalt biz nady sabafia fortjuleben,

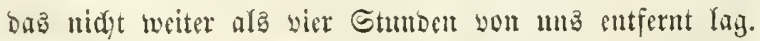

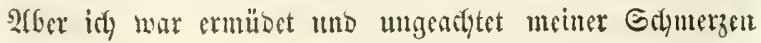
ppürte idf einen ziemlid) gnten 2(ppetit. Soly bejtimmte 


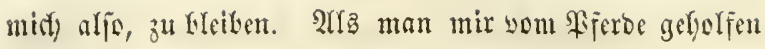

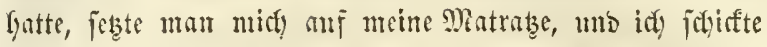
mid) an, meinen 2(ntlyeil vou Mlittagsecfien cingunefyen.

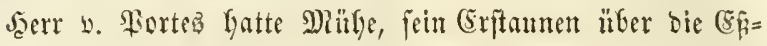
luift, melete idf jeigte, ju verbergen; bem in ber That, id

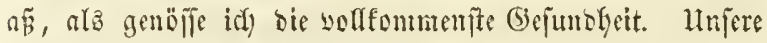

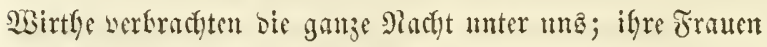

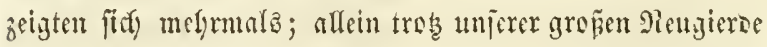

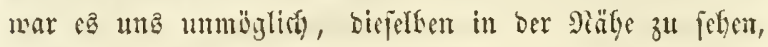

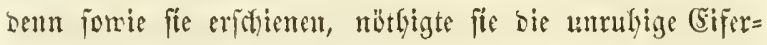

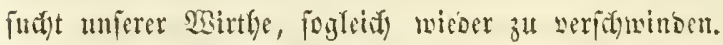

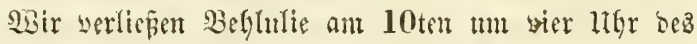

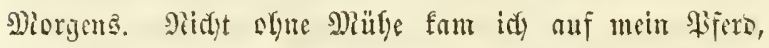

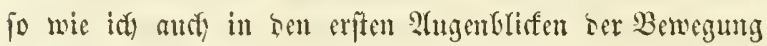

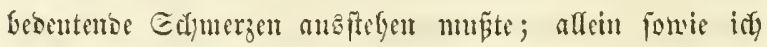

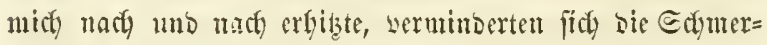

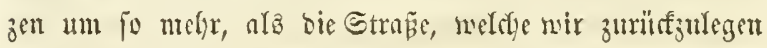

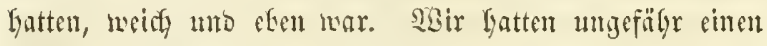

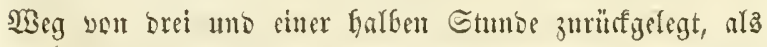

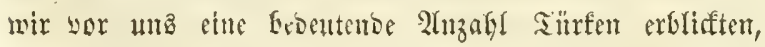
melde alle berafinnet maxen uro zientich idyöne arabifde Fferbe reitend, lleburgen mit ber Ranze vormafymen.

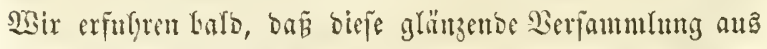
bem Minfafitt (3olloireftor) und feinen Dipicieren beftanto. So wie fie uns erbliften, beeilten fie fid fommen und unjere PFerbe zu Getrataten. Die Füllen jojie=

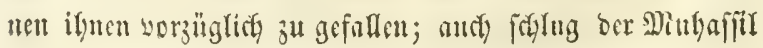




\section{1}

Dem Etallnteifter vor, cimes wou ifyen gegen cin Fiferd

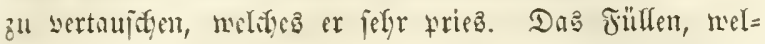
des jiute Beyierie crregte, mar gerabe der Mlélén, Gohn Des 2tfou=\$gant. 2flein biejes junge Thier fereditigte

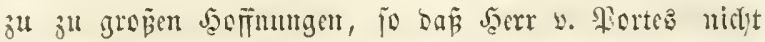
Daran Dadyte, ez afjutreten. (Ẽr jolug afer vor, ilym

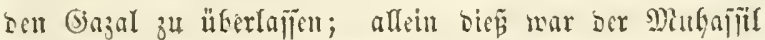
micber nidat zufrieden, welder unz barauf oas \$rers jefen liep, meldes er vertaujact wollte. Dajielfe mar

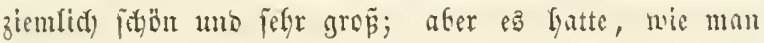
jagt, gebunsente edfultern, jomic jelgr fleime und jefjr

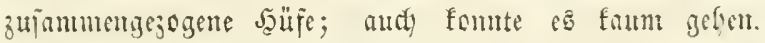

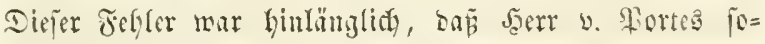

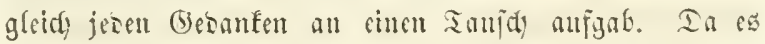

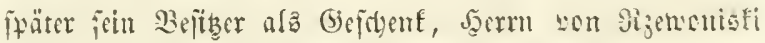

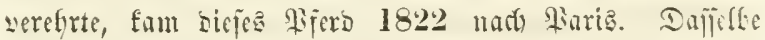
geförte bamals zu eincm Trangport vou viermingmalig

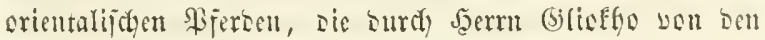

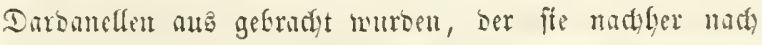

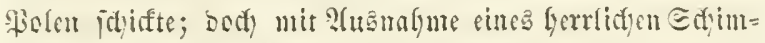

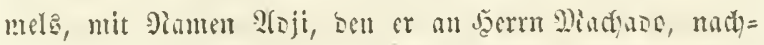

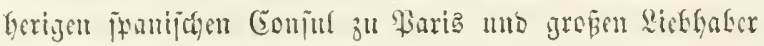
arabijat)er Hierbe, verfaufte.

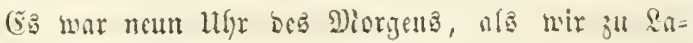

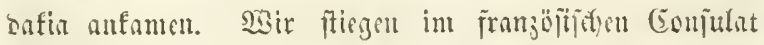

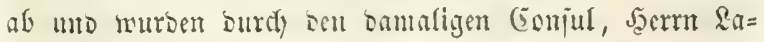

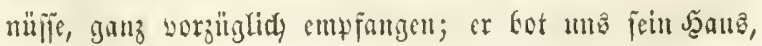


- wie jeme sajal an, uno wir hüteten uns, wie mun

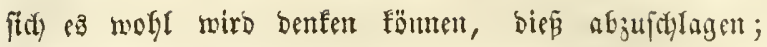
felbjt unjere Sfferde lagerten int

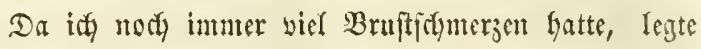
idi) mids, gleidy nadyom id) wom Siferbe geftiegen war,

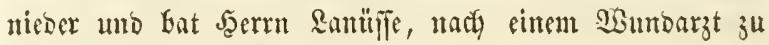
jedyiffen, un nir 2loer zu lajien. Diejer beriprach mir,

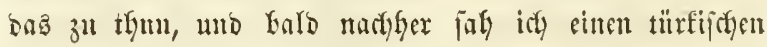
Barbier mit einem langen Barte erjaciuen; er batte cine

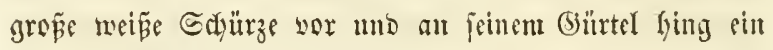

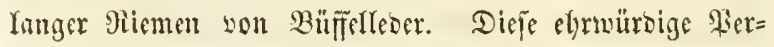

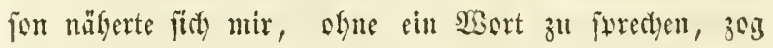

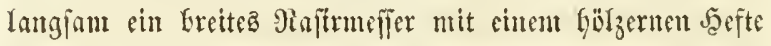

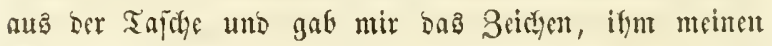

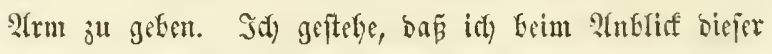
fonberbaren \&anzette, welde er in ber.ఏanb bielt, erjarat;

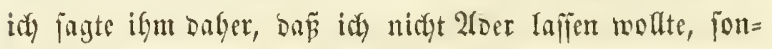
Dern ígn nur um Das Anlegen won Bhutegeln auf bie Bruft bäte. Itrzmeifelgaft mar es aber sas erfte Mial,

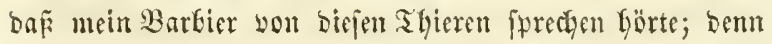
er fofien mich nidut zu serfeften und jagte mir nun, Daß́,

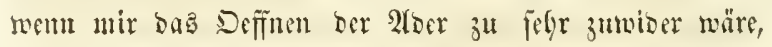

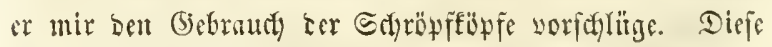
Speration folien mir weniger gerägrlich, als ein tïrfijcher

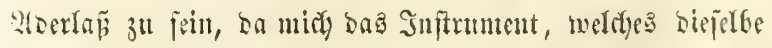
verricten follte, nidytä weniger als ciner Serlebung oer Siulaber autejeste. Ser milligte aljo ein, und da ev 


\section{3}

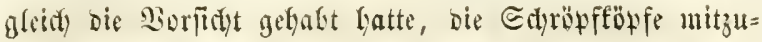
bringen, jo feste or mir brei auf jebe Bruftjeite, zümbete barauf ein menig Baumwolle an, mu bem luftleeren Baum z̆ bemirfen, und als er jie abgomommen batte,

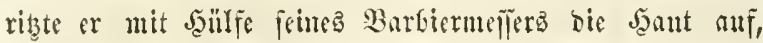

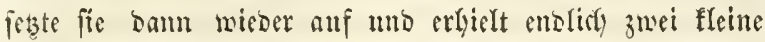
örtlithe 2(berläfie, meldi)e nir jebod) mofl thaten. Dem barauf folgeneen Tag bedertte er mir bie ganze $\mathfrak{B r u f t}$ mit

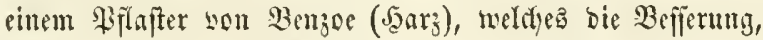
bie idf) jeit worigem ?(Gento werpürte, auf cine merfficf) 2(rt sermelyte utb mid) pogar in bon Stand jetete, gefent

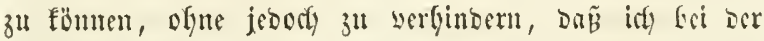

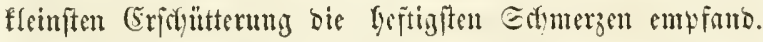

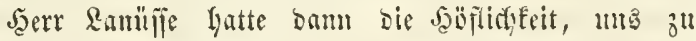
tritten, แađ cinige Tage länger bei ifm zu bleiber, als

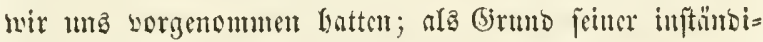
gen Bitten gab er ben Tag bez Geilizen \&ubnig, weldyer Yeramafyete, und bie Miotfwendigfeit der ungeftörteften Siutge an, bie tidy nöthig lyatte, um wolltommen wieber Gergcifellt zul merben. Der Etallmeifter milligte ein, unb

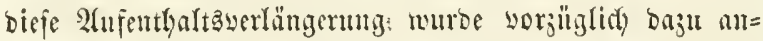

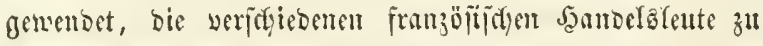
bejudyen, weldye fitd zu Rabafia niedergelafien batten, mo

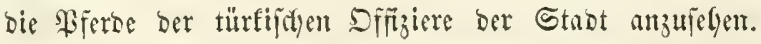
Bei cintem biejer (bänge fragte mich ein Saetr Maritta, cin Franjoje, ben ber Mangel foficflicter Beuge und cinez europailidyen Edyneibers cine nogl werĩefene Barberobe zu 


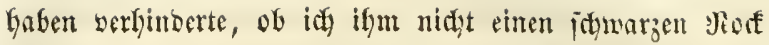

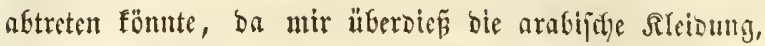
welde id beftündig trüge, biefen Theil ber Garberobe

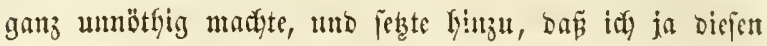
Mangel bei meiner Burüffiuft in Franfreich Ieidut mürbe

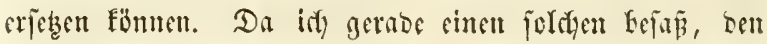

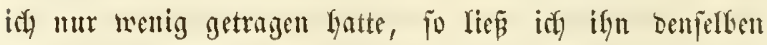

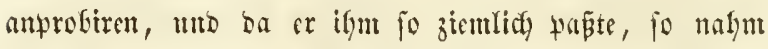
or ifgn fïtr umgefägr zelgn Pfund Srommonicuthar wor

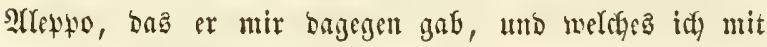
uad) Paris Gradtete.

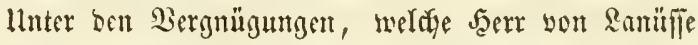

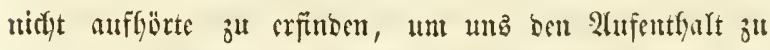

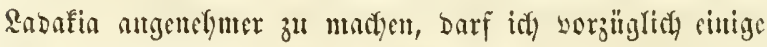
Fijefereien nitgt vergefien, bie wir an ber Dieeresfuifte

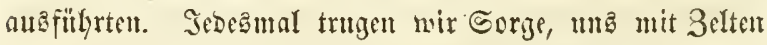
ju werfeften, fowie and mit allem Miöthigen, um bie

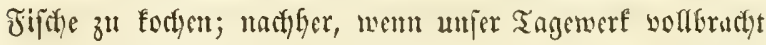

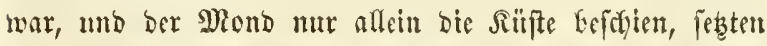
nir uns lufftig zur Tafel, und oft verlängerte fid bus Miafl bis in bie Matyt. 2(ucly gab er mes melgrmals

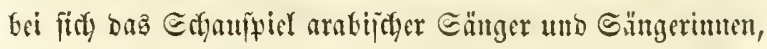
melelye bei Dem Rlange ber umfarmonificten Mlufte biefes Randes friegerifdye Tänze ansfitlyten, die für bie rişer

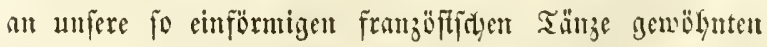
Sugen niçt einer gewifien 2 (tumuth) erntangelten.

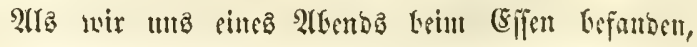




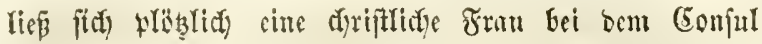

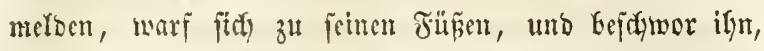

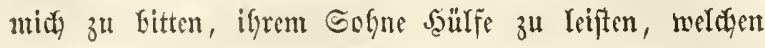

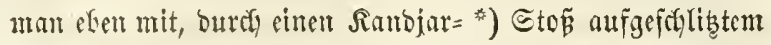

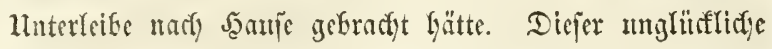
junge Mam follte fith ber folgenden Tag verbeiratgen. An Tage war or mit jungen Türten baben gegangen.

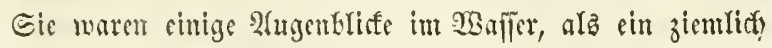

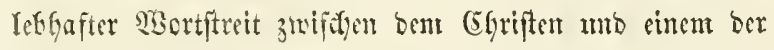
Ninjefmämer entipant; biejer Reştere wartet bie Beenbi=

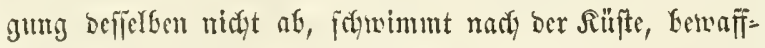
net fich nit feinem Sanbjar, ftürgt fiti) weder in bas Meer, näfert fitd feinem Segner, taud) unter, unb unter

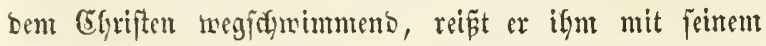
Doldye ben Unterteib auf tmb entfliegt. Die andern Tärfen thatem bajielfe. Das fo verlafijene Dpjer fyatte jebon nod) fo wiel Seäfte, um bas Miccr verlaffer zu

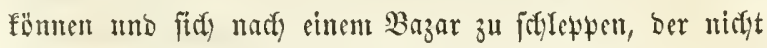
weit yon ber flarfen umb fanbigen Rïlite entfernt mar; afer mur hier angefommen, janf er erifüpft nieber. Cin

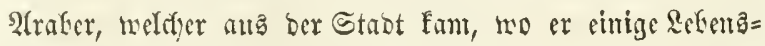

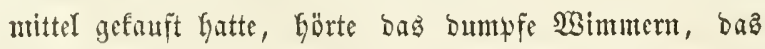

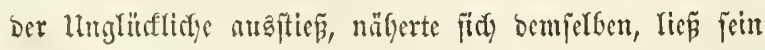
Sameel nifberlegen, hob ben Sitanfen barauf uno Gradite

-) Der Sanbjar iff cine Ifrt Doldy, Ser fefre Iang, bebeus tent breit, gerogen und zweifdneibig ift. 
ifn fo in Das Şans feiner Mutter. Man wirb ben

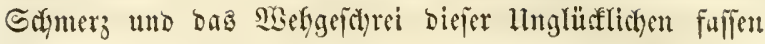

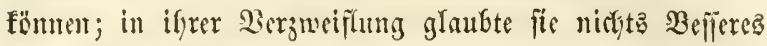

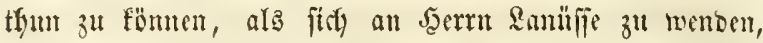

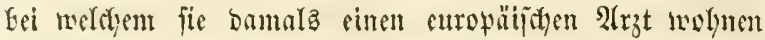

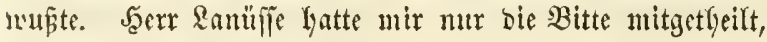
bie man an ign geridytet lyatte, als idy, ofwe cinen 2 u= genbliff zu serlieren, midt) mit meinem Bindezeug veriats und mit ifut in bie Niobumng bes Spfers eilte.

Raum angefonmen, füfrte man midt) zu bem sod= Eranfen, Der auf ciner Miatte ausgejtrefft in einer 2lrt Sebenzimuter lag, worim man zmei ungeheure Rohylenpfanmen, mit Roblen im sollen Brantse angefïllt, aufgeftellt batte, fomiz orei oser wier Sidyter, seren Dorfte ben Erlöjtyen

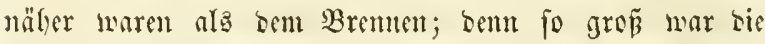

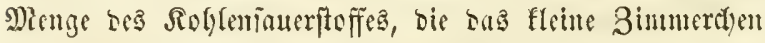
crfïnte. Sier Türfen umgaben ifn, melche Gemülyt maren, bie Manje won Geoärmen, die Geraus getreten war, in

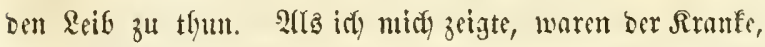
fomie biejenigen, welabe ifyn ungaben, malye saran,

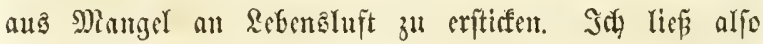
ben Türfen jagen, auf bas fornellite binansengelyen, sie

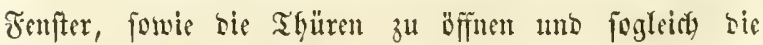
Roblen Gimams zu idyaffen. Ars man alle bieje Sors

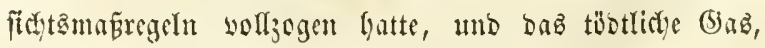
melifes vorfer ธaร Bimmer erfüllte, weriftummben mar, trat idy cin unb fing an sen Siranfen ju unterifuden, 


\section{7}

Der Unterleib bieję Unglüffidjen war voller Sand; feite Siebärme twaren fobwary uno an mefren Stellen

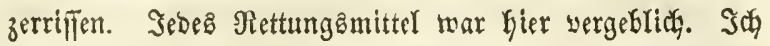
fagte ę Serrn Ranüfĭe, ber midf jeboch bat, bie Maje Bebarnte wieber in ben Hutterleib zu Yegen unb bie Şaut= nabt zu madjen, um bei biejem Unglüdticfen nidgt ben fürdyterlidyen Bebanfen auffommen zh Iajpen, als wolle

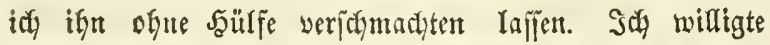
eit, nalm eitren Sawamnt, ben iđ in mit etwas Iauem

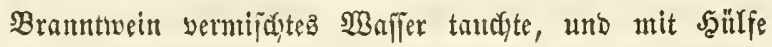
beffelben nafm ich bie fremben Theile, bie fich am Darm= felle feitgefebt Gatter, Keraus; barauf Iegte ich bie be= bärne wieber an ifre Gtelle und nähete bann bie Scaut zu.

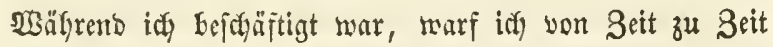

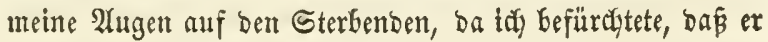
unter meinen Saänben ben Beift auşaudyen nürbe. Der

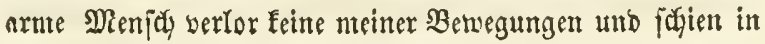
nteinen Bliafen ben Sebanken zu Yejen, ber midh bejugäfigte.

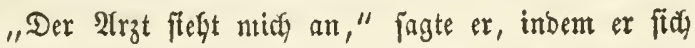
ă feiner Slutter und ben üfrigen limptefenden renbete, "aber er foll nidytō fürchten, iç fterbe niçyt, ba er fid meiner angenommen bat."

Nadibem bie Operation beenbigt mar, fragte er nich, of id ifm molgl erlauben wolle, Etwaz zu efien;

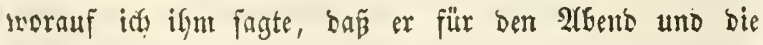

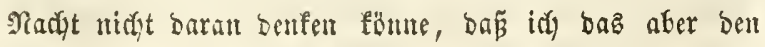
anoern হing, ment ich ifgn wieber beftude, feben würbe; 


\section{8}

er reifhte nir bie Sand unb brünte bie meinige fräfig, inbent er midy bat, ign nidat zu verlafien. Şierauf ging

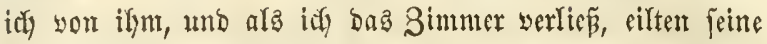
Sermanten ju mir, füpten mir bie Şänbe und fragten

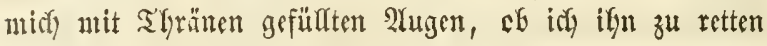

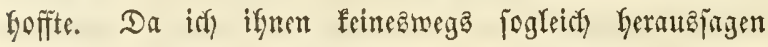

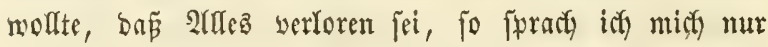

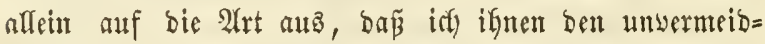

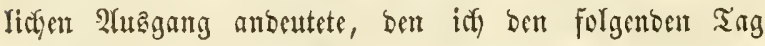
vora!şaly. Der 2rat mit Den Sdyröpfföpfen war aud gerufen worben, und biefen veranlabte ids, ben anderu Morgen zu mir zu fommen, um mir zu meloen, mie eв mit bem franfen ginge.

Ciefen Uhr bes Mlorgens mar ç, als ber püntt= licte Sarbier zu mir faut unb fith mir zu jagen becilte, Dáp fich ber Sranfe gebeffert, bie গacht wollfommen gut yollbractyt Gätte, unb midy inftänbig zu feben verlangte. Sach begate ntich fogleich zu ifm, und fobalo er meiter an= fitctig murbe, fagte or mir, bá er bie ganze Nadyt feine

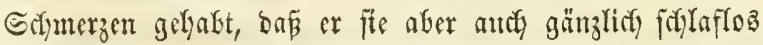
zugefradyt hätte; or febste Gimgu, dá er hungrig wäre,

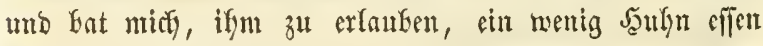

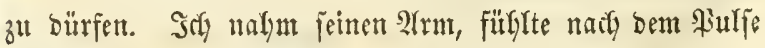
uns unterjucte alle übrigen Theile: Dor Puls war nicht mefr füblbar; bie entfernteften Rörwertbeile waren

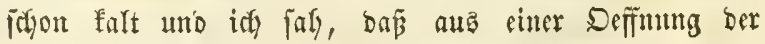
SYumbe, welche ich mit Freíp gelaffen Gatte, eine grop̃e 


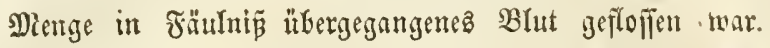

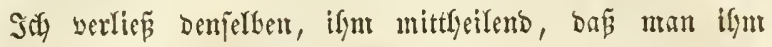

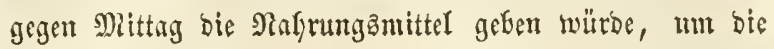
er midy gebeten hatte.

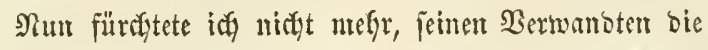

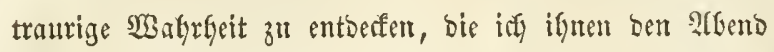
vorber verforgen hatte, und rieth benjelfen, ign auf bas

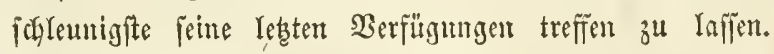
Dieje Beroronung fam ž rechter Beit, Demr noch benfelleen Dorgen gegen zefa ugr gab er feimen beift auf.

Sein Mörber murbe eingezogen und yor bent Radi

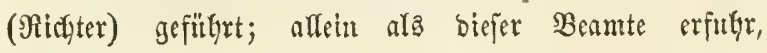

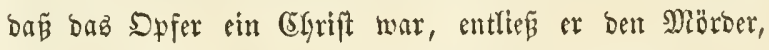

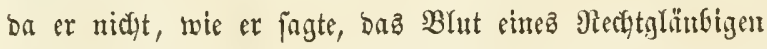

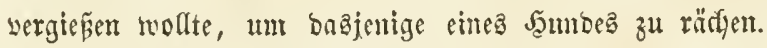

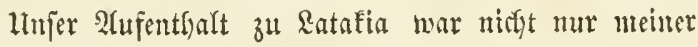
(bejumbleit zuträglifi, fondern auch für umpere Pferbe gümftig. So gatte ber 2 tbout $=2 \mathfrak{2 r f o u b}$ in Gefirge bie

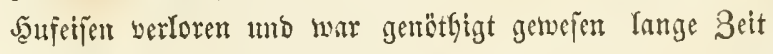
Garfü zu gelyen; in Folge beffen Gatte er werichlagen umb

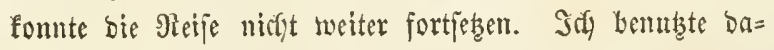

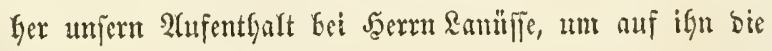
nötfige Corgfalt zu verwenben, fomie ifgn geförig jube= iđglagen, uno, Danf ber Nuhye, weldye er hier fauto, bemt er founte ber Raramane folgen, als enolich ber 2fttgen=

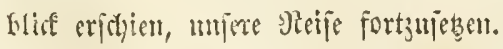


Drud von ฐ. $\mathfrak{B}$. seirfigfelo in Reipzig. 



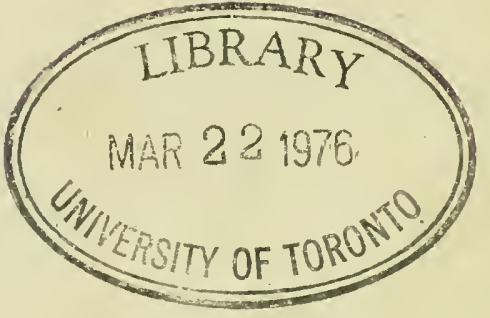




\section{PLEASE DO NOT REMOVE}

CARDS OR SLIPS FROM THIS POCKET

\section{UNIVERSITY OF TORONTO LIBRARY}


